\title{
Connected Coverage with Rapid Forwarding in Energy Harvesting Wireless Sensor Networks for Critical Rare Events
}

by

\author{
David C. Harrison
}

A thesis

submitted to the Victoria University of Wellington in fulfilment of the requirements for the degree of

Doctor of Philosophy in Engineering.

Victoria University of Wellington 2017 



\begin{abstract}
To ensure event detection and subsequent rapid forwarding of notification messages, wireless sensor networks deployed to detect critically important rarely occurring events must maintain both sensing coverage and low latency network connectivity at all times.

Maintaining coverage for extended periods is relatively straight forward as passive sensing components tend to consume little energy. Maintenance of network connectivity, however, requires sensing devices constantly supply power to their transceivers, significantly reducing the longevity of the sensor network.

Energy harvesting can extend the operational life of sensing devices with always on transceivers, potentially to the point where they can operate year round. In addition, over populating the sensing area with more devices than are required to provide complete sensing cover introduces the possibility of self-organisation where sensing devices agree amongst themselves which will remain active and which will be allowed to sleep.

Few algorithms have been proposed to address both coverage and forwarding; those that do are either unconcerned with rapid propagation or have not been optimised to handle the constant changes in topology observed in duty cycling networks.

This thesis first analyses the energy consumption profiles of commercially available wireless sensing devices then presents mechanisms by which these devices can both maintain sensing coverage and rapidly forward event detection messages delayed only by the inherent latencies found in wireless multi-hop networks. These individual contributions form the basis of a combined algorithm for Coverage Preservation with Rapid Forwarding (CPRF).

Through evaluations including live deployment, CPRF is shown to deliver perfect coverage maintenance and low latency message propagation whilst allowing stored-charge conservation via collaborative duty cycling in energy harvesting networks.
\end{abstract}




\section{Contents}

$\begin{array}{ll}\text { Publications } & \text { xiii }\end{array}$

$\begin{array}{ll}\text { Glossary } & \text { xv }\end{array}$

1 Introduction 1

1.1 Synopsis ......................... 4

1.2 Contributions .................... 6

1.3 Outline ....................... 7

2 Related Work 9

2.1 Event Sensing Strategies . . . . . . . . . . . . . . . . . . . . . . . . . . . . . . . . .

2.1.1 Collaboration ................... 14

2.1 .2 Duty Cycling . . . . . . . . . . . . . . 16

2.1.3 Component Deactivation . . . . . . . . . . . . . . 17

2.1.4 Over Population / Node Redundancy . . . . . . . . . . . . . 19

2.1.5 Message Suppression and Data Aggregation . . . . . . . . . 20

2.1.6 Burst Aware Protocols . . . . . . . . . . . . . . . . 20

2.1 .7 Always On . . . . . . . . . . . . . . . 22

2.1.8 Energy Harvesting . . . . . . . . . . . . . . 23

2.1.9 Classification of Existing Work . . . . . . . . . . . . . 24

2.1 .10 Discussion . . . . . . . . . . . . . . 27

2.2 Coverage and Connectivity Algorithms . . . . . . . . . . . . . . 31

2.2.1 Span: Energy-efficient Topology Maintenance . . . . . . . . 32

2.2.2 CCP: Integrated Coverage and Connectivity Configuration . 35

2.2.3 RACP: Role Alternating Coverage Preservation . . . . . . . 37

2.2.4 OGDC: Optimal Geographical Density Control . . . . . . . . 38

2.2.5 Sleep+PC: Distributed Coverage and Connectivity . . . . . . 40

2.2.6 DLM: Distributed Lifetime Maximisation . . . . . . . . . . . 42

2.2.7 CAS: Optimized Event Detection . . . . . . . . . . . . . . . 44 
2.2 .8 Other Algorithms . . . . . . . . . . . . . . . 46

2.2 .9 Discussion . . . . . . . . . . . . . . . 47

2.3 Geographic Forwarding Algorithms . . . . . . . . . . . . . . . 49

2.3 .1 Collision Avoidance . . . . . . . . . . . . . . . . . 50

2.3.2 Energy Conservation . . . . . . . . . . . . . . . . . . . . . . 51

2.3 .3 Hole Avoidance . . . . . . . . . . . . . . . . . . 53

2.3 .4 Discussion ...................... 53

2.4 Summary ............................ 54

3 Power Consumption $\quad 57$

3.1 Evaluation Setup . . . . . . . . . . . . . . . . . . . 57

3.2 Measuring Current Draw . . . . . . . . . . . . . . . . . . . . . . 58

3.3 Transceiver Warm Up . . . . . . . . . . . . . . . . . . . . 60

3.4 Idle Listen vs Transmit \& Receive . . . . . . . . . . . . . . . . . 60

3.5 Effect of Reducing Transmit Power . . . . . . . . . . . . . . . . 62

3.6 Device Differences . . . . . . . . . . . . . . . . . . . . 62

3.7 Power Consumption and Energy Harvesting Model . . . . . . . . . 63

3.8 Impact on Existing Research . . . . . . . . . . . . . . . . . . 65

3.9 Summary ........................... 66

$\begin{array}{lll}4 & \text { Equitable Sleep Coverage } & 67\end{array}$

4.1 Coverage Preservation Algorithm . . . . . . . . . . . . . . . 68

4.1 .1 Overview ...................... 68

4.1 .2 Sleep Eligibility . . . . . . . . . . . . . . . . . . . . . . . . . . . . . . 72

4.1 .3 Node Placement . . . . . . . . . . . . . . . . 73

4.2 Evaluation ........................... 76

4.2.1 Energy Model \& Simulation Parameters . . . . . . . . . . 76

4.2.2 Algorithm Efficacy . . . . . . . . . . . . . . 77

4.2.3 Required Charging Efficiency . . . . . . . . . . . . . . . 79

4.2.4 Comparative Study . . . . . . . . . . . . . . . . . 80

4.2.5 Non-Uniform Sensing Ranges . . . . . . . . . . . . . . . . 83

4.2.6 Implementation \& Simulated Energy Harvesting . . . . . . . 84

4.2.7 Simulation and Implementation Equivalence . . . . . . . . . . . . . . . . . . . . . . . . . . . . . . .

4.3 Summary . . . . . . . . . . . . . . . . . . 89 
5 Swift Opportunistic Forwarding $\quad 91$

5.1 General Approach . . . . . . . . . . . . . . . . . . . 91

5.2 Message Suppression \& Collision Avoidance . . . . . . . . . . . . 93

5.3 Event Reliability . . . . . . . . . . . . . . . . . . . . . . . . . . . . . . 95

5.4 Event De-Duplication . . . . . . . . . . . . . . . . . 96

5.5 SOFIE Pseudo Code . . . . . . . . . . . . . . . . . . . . 98

5.6 De-Duplication Evaluation . . . . . . . . . . . . . . . . . 99

5.7 Comparative Evaluation . . . . . . . . . . . . . . . . . 100

5.7 .1 Delivery Ratio . . . . . . . . . . . . . . . . . 102

5.7 .2 Fixed Topology . . . . . . . . . . . . . . . . . 104

5.7 .3 Variable Topology . . . . . . . . . . . . . . . . . 104

5.7 .4 Optimum ratio of $R_{c}$ to $R_{s} \ldots \ldots \ldots 108$

5.8 Summary . . . . . . . . . . . . . . . . . 113

6 Coverage Preservation with Rapid Forwarding 115

6.1 Summary of Related Work . . . . . . . . . . . . . . . . . 116

6.2 Algorithms Chosen for Comparison . . . . . . . . . . . . . 118

6.3 Combined Algorithm . . . . . . . . . . . . . . . . . . 118

6.3 .1 Rapid Forwarding . . . . . . . . . . . . . . 119

6.3 .2 Sleep Eligibility . . . . . . . . . . . . . . . . . . . . . 119

6.3 .3 Sponsor Group Selection . . . . . . . . . . . . . . . . 120

6.3 .4 Neighbour Selection . . . . . . . . . . . . . . . . 121

6.3 .5 Metric Equality . . . . . . . . . . . . . . . . 121

6.3 .6 Sleep Scheduling . . . . . . . . . . . . . . . . . . 122

6.3.7 The Sleep Eligibility "Daisy Chain" . . . . . . . . . . . . . . . 128

6.4 Evaluation . . . . . . . . . . . . . . . . . . . . 129

6.4 .1 Sleep Eligibility . . . . . . . . . . . . . . . . . . 129

6.4 Coverage Preservation . . . . . . . . . . . . . 130

6.4 .3 Rapid Forwarding . . . . . . . . . . . . . . 138

6.5 Implementation : Test Bed \& Live Deployment . . . . . . . . . . . . 143

6.6 Summary . . . . . . . . . . . . . . . . . . 147

7 Conclusions 149

7.1 Review ........................ 150

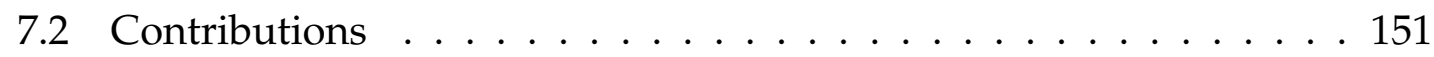

7.3 Future Work . . . . . . . . . . . . . . . . . . 152 


\section{List of Figures}

1.1 Light poles on the M62 in England . . . . . . . . . . . 5

2.1 Span: Fraction of nodes remaining as a function of simulation time. 33

2.2 Span: Percentage of time spent as a coordinator . . . . . . . . . . . . 34

2.3 An example of 1 -coverage eligibility . . . . . . . . . . . . . 35

2.4 CCP: Coverage . . . . . . . . . . . . . 36

2.5 CCP: Connectivity . . . . . . . . . . . . . . 37

2.6 RACP: Coverage ratio . . . . . . . . . . . . . . 38

2.7 OGDC: 45 hexagons required to cover a $50 \mathrm{~m}^{2}$ square sensing area . 39

2.8 OGDC: Sensing coverage . . . . . . . . . . . . . . . . . 40

2.9 OGDG: $\alpha$-lifetime against $\alpha \ldots \ldots$. . . . . . . . . . 40

2.10 Sleep+PC: Coverage . . . . . . . . . . . . . . . . . . 41

2.11 Sleep+PC: Connectivity . . . . . . . . . . . . . . . . . 42

2.12 DLM: Target field in a small WSN . . . . . . . . . . . . . . . . . . 43

2.13 DLM: Normalized lifetime versus error index . . . . . . . . . . . . . 43

2.14 CAS: Detection delay . . . . . . . . . . . . . . . . . . . 45

2.15 CAS: Detection probability . . . . . . . . . . . . . . . 46

3.1 Current draw measurement circuits . . . . . . . . . . . . . . . . . 59

3.2 Voltage drop across resistor shunt for timer based activation of the CC2420 transceiver in an MTM-CM5000-CSP. . . . . . . . . . . . 61

3.3 Voltage drop across resistor shunt receiving a 14 byte packet. . . . . 61

3.4 Voltage drop across resistor shunt sending 14 byte packet . . . . . . 62

3.5 Voltage drop across resistor shunt responding to a timer. . . . . . . 62

4.1 ESCARGO State Transitions . . . . . . . . . . . . . . . . . 69

4.2 Coverage eligibility. . . . . . . . . . . . . . . . 73

4.3 Placement of nodes relative to sensing area . . . . . . . . . . . 74

4.4 Nodes with at least one sponsor group by deployment density. . . . 75

4.5 Mean stored charge during first month of operation. . . . . . . . . . 77 
4.6 Sensing coverage \& node state, battery power with ESCARGO. . . 78

4.7 Mean stored charge over one calendar year, 70\% efficient charging. 79

4.8 Mean stored charge over time by charging efficiency. . . . . . . . . . 80

4.9 Placements for comparative study and evaluation of implementation 81

4.10 Time of initial coverage loss by algorithm . . . . . . . . . . . 82

4.11 Percentage sleep time by algorithm . . . . . . . . . . . . . . . 82

4.12 Random distribution of 52 nodes . . . . . . . . . . . . . . . 83

4.13 Test environment with 59 nodes from Figure $4.9 \mathrm{~b}$. . . . . . . . . . 85

4.14 Implementation: Sleep times converging rapidly after initialisation. 86

4.15 Stored charge is equalised in initial non harvesting period . . . . 86

4.16 If a node restarts, its sleep time rapidly converges back to the mean. 87

4.17 Stored charge over first 48 hours of operation. . . . . . . . . . . . 87

4.18 Stored charge over one simulated calendar year. . . . . . . . . . . . 89

5.1 Comparison of forwarding regions. . . . . . . . . . . . . . 92

5.2 Possible forwarding paths. . . . . . . . . . . . . . . . . 94

5.3 SOFIE event de-duplication . . . . . . . . . . . . . . 96

5.4 Deployment density . . . . . . . . . . . . . . . . . . . 99

5.5 Delay improvement, PHY receive errors, \& event de-duplications . 100

5.6 Sensing node placements providing 100\% sensing area coverage. . 102

5.7 Delivery ratio by node density. . . . . . . . . . . . . . . . 103

5.8 Notification Delay . . . . . . . . . . . . . . . . . . . . 105

5.9 Receive Errors . . . . . . . . . . . . . . . . . . . . 106

5.10 Energy Consumed in non idle transceiver states . . . . . . . . . . . 107

5.11 Notification delay, coverage preserving, sleep-scheduled network · 109

5.12 Hops taken, coverage preserving, sleep-scheduled network . . . . . 110

5.13 Notification delay, planned placements, decreasing $R_{s}$ for fixed $R_{c} \cdot 111$

5.14 Hops taken, planned placements, decreasing $R_{s}$ for fixed $R_{c} \ldots \ldots 112$

6.1 Unique, non-redundant sponsor groups . . . . . . . . . . . . . . 120

6.2 CPRF State Transitions . . . . . . . . . . . . . . . . . . . . 123

6.3 Sleeping nodes by increasing deployment density . . . . . . . . . . 130

6.4 Sensing Coverage in Dense Deployment . . . . . . . . . . . . . . . . 131

6.5 Stored Charge in Dense Deployment . . . . . . . . . . . . . . . . . . 132

6.6 Sleeptime in Dense Deployment . . . . . . . . . . . . . . . . . . . 133

6.7 Stored Charge in Sparse Deployment . . . . . . . . . . . . . . . . . . 134

6.8 Sleeptime in Sparse Deployment . . . . . . . . . . . . . . . . 135 
6.9 Sensing Coverage in Sparse Deployment . . . . . . . . . . . . . . 136

6.10 Minimum sleeptime . . . . . . . . . . . . . . . . 137

6.11 Comparison of forwarding regions . . . . . . . . . . . . . 140

6.12 Delivery ratio, notification delay, and forwarding hops . . . . . . . 142

6.13 Notification delay distribution . . . . . . . . . . . . . . . . . . . 144

6.14 Evaluation Environments ． . . . . . . . . . . . . . . . . 145

6.15 UCMote Mini powered by simple photovoltaic energy harvester . . 146

6.16 Simple photovoltaic energy harvester schematic . . . . . . . . . 146 


\section{List of Tables}

2.1 Most Cited WSN Surveys Since January 2010 . . . . . . . . . . . 10

2.2 Volume of Surveyed WSN Research on Rare Events . . . . . . . . 12

2.3 Typical Effects of Sensing Strategies . . . . . . . . . . . . . . . 13

2.4 Current Draw for WSN Components . . . . . . . . . . . . . . . 19

2.5 WSN Research on Rare Event Sensing . . . . . . . . . . . . . 25

2.6 Range of WSN Components . . . . . . . . . . . . . . . . . . 32

2.7 Coverage \& Connectivity Algorithms . . . . . . . . . . . . 48

2.8 Typical Effects of Forwarding Priorities $\ldots \ldots \ldots \ldots$

3.1 Current Draw by Measurement Technique . . . . . . . . . . . 60

3.2 Solar Panel Specifications . . . . . . . . . . . . . . . . . . . . 64

3.3 Advanticsys CM5000 Simulation Parameters . . . . . . . . . . 64

4.1 Sponsor Groups for Nodes in Figure 4.3a $\ldots \ldots \ldots \ldots$

4.2 Sponsor Groups for Nodes in Figure 4.3b . . . . . . . . . . . 75

4.3 IEEE 802.15.4 CSMA/CA Parameters . . . . . . . . . . . 76

6.1 Sleep Eligibility - Daisy Chaining . . . . . . . . . . . . . . . 128

6.2 Algorithm Settings . . . . . . . . . . . . . . . . . . . . 129

6.3 Detection Delay for Randomly Located Events . . . . . . . . . . 139

6.4 Implementation Evaluation Results for 1,000 Messages . . . . . 146 


\section{Publications}

The following short paper is derived from Chapter 4:

- David C. Harrison, Winston K. G. Seah, and Ramesh K. Rayudu. “Coverage Preservation in Energy Harvesting Wireless Sensor Networks for Rare Events". Proceedings of 40th Conference on Local Computer Networks (LCN). IEEE, October 2015. [1]

The following survey paper forms the basis of Section 2.1:

- David C. Harrison, Winston K. G. Seah, and Ramesh K. Rayudu. "Rare Event Detection and Propagation in Wireless Sensor Networks". ACM Computing Surveys. Volume 48, No. 4, Article 58. March 2016. [2]

The following letter is derived from Chapter 3:

- David C. Harrison, Winston K. G. Seah, and Ramesh K. Rayudu. Busting Myths of Energy Models for Wireless Sensor Networks. Electronics Letters. IET. Volume 52, Issue 16, p. 1412-1414, featured p. 1359, August 2016 [3]

The following short paper is derived from Chapter 5:

- David C. Harrison, Winston K. G. Seah, Hang Yu, and Ramesh K. Rayudu. "Opportunistic Geographic Forwarding in Wireless Sensor Networks for Critical Rare Events". Proceedings of 41st Conference on Local Computer Networks (LCN). IEEE, November 2016 [4]

The following paper derived from Chapter 4 has been modified and re-submitted for publication after initial feedback from the journal editors:

- David C. Harrison, Winston K. G. Seah, and Ramesh K. Rayudu. “Coverage Preservation through Equitable Sleep Scheduling in Energy Harvesting Wireless Sensor Networks for Critical Rare Events". IEEE Transactions on Industrial Informatics - Special Section on Internet-of-Things for Smart Cities and Urban Informatics. 
The following paper derived from Chapter 6 has been modified and re-submitted for publication after comprehensive feedback from the journal editors:

- David C. Harrison, Winston K. G. Seah, and Ramesh K. Rayudu. “Coverage Preservation with Rapid Forwarding in Energy Harvesting Wireless Sensor Networks for Critical Rare Events". ACM Transactions on Embedded Computing - Special Issue on Autonomous Battery-Free Sensing and Communication. 


\section{Glossary}

Terms used in this thesis are defined or cited at first use. Heavily used terms and presented in this table for ease of reference:

Term

\begin{tabular}{ll}
\hline Communication Range & Maximum distance a WSN device can transmit a message \\
with acceptable certainty of it being received. Marked as & $R_{c}$ in this manuscript. \\
& A rare event of considerable importance. Detection must \\
be guaranteed and notification delay must be minimized. & In the most difficult situations, the event is ephemeral in \\
the extreme, and entirely transitory. & \\
Critical Rare Event & Time take for notification of an event occurrence to reach \\
the sink. Composed of the time taken for the node to pro- \\
cess sensor data and construct a notification message, and \\
the time taken for that message to propagate to the sink. \\
Detection Delay \\
Likelihood an event will be detected. For critical rare \\
events, all events must be detected. \\
Situation specific occurrence of sufficient importance to \\
warrant sending a notification to the sink. Events can be \\
ephemeral (their occurrence is fleeting), transitory (they \\
leave no trace of their occurrence), or both.
\end{tabular}

\section{Definition}

Continued on next page... 
Event Sensing

Hop

Network Connectivity

Node

Notification Delay

Rare Event

Sensing Coverage

Sensing Range

Sink

Sleeptime
Style of WSN deployment were messages are forwarded to the sink when they occur, or shortly thereafter. Distinct from the more common periodic sampling and query based styles.

In networks were some nodes are beyond communication range of the sink, those nodes can have their messages forwarded by geographically intermediate nodes. Each transmission of a message is regarded as a hop.

A network is connected if all active nodes in the network can forward a message to the sink.

A modest capability computing device in the WSN. Minimally contains a MCU, a small amount of RAM, one or more sensors and a radio transceiver.

The major component of detection delay, the time taken for an event notification message to propagate from the originating node to the sink.

An event that occurs infrequently, the definition of infrequent being situation dependent.

An arbitrary area is sensing covered is every point in that area is within sensing range of at least one active node.

Maximum distance at which nodes can reliably sense an event. Marked as $R_{s}$ in this manuscript.

A transceiver equipped device regarded as the exit point of the WSN. Often mains powered and connected to a wired network and/or the Internet.

Time (usually \% of time) nodes are "asleep" i.e. the combined duration of all periods they are powered down into their lowest energy state - a state from which the device will be programmatically woken at some point in the future. 
xvii

Term

Stored Charge

\section{Definition}

Energy reserve of the node, typically expressed in $m A h$. A node with no stored charge is "dead". Nodes typically have either an initial charge that depletes over time (battery power) or charge is replenished via energy harvesting up to some maximum capacity. 


\section{Chapter 1}

\section{Introduction}

Wireless sensor network (WSN) nodes are typically small battery powered or energy harvesting devices consisting of a micro controller, a small amount of random access memory, possibly some non-volatile storage, one or more sensors, and a low power radio transceiver which is usually the most power hungry component. The finite charge storage capacity of batteries and the limited capabilities of small scale energy harvesting devices shapes WSN research to the extent that minimising energy consumption becomes a preoccupation; the less energy consumed, the longer the network will continue to operate.

The simplest way for a sensing node to conserve energy, and in doing so maximise its operational life, is to power down for extended periods, either entirely or just by removing power from its transceiver. For star topology networks where sensing nodes are connected to a permanently powered base station, each node can adopt an independent duty cycle and a media access control (MAC) protocol based on un-slotted carrier sense multiple access with collision avoidance (CSMA/CA). Nodes deployed to periodically sample data for multi-hop transmission to a base station can synchronise their activity [5] to ensure network connectivity and employ algorithms that minimise overuse of individual routing nodes [6]. Similar techniques can be adopted for networks where data collection is initiated by a request from the base station [7]. Sensing rare events, however, introduces additional complexity.

Rare events are axiomatically situations that occur infrequently; they may also be short-lived, present themselves unpredictably and leave no trace of their presence when complete. For the purposes of this work, rare events are considered random as those following a predictable schedule can easily be sensed assuming 
accurate time synchronisation [8]. Successfully sensing a rare event requires consideration of the extent to which it is ephemeral and transitory. A scheme proved effective for sensing forest fires in progress may not be as successful detecting the instantaneous start of the same fires. Similarly, a scheme for detecting perimeter intrusion on a battlefield where events last fractions of a second and leave no discernible trace may prove inappropriate for sensing landslides that last for comparatively extended periods and leave significant physical evidence in their wake, yet occur less frequently.

A study of rare event simulation using Monte Carlo Methods [9] compliments an earlier work on estimating rare event probabilities [10] and defines a rare event as:

"an event occurring with a very small probability, the definition of 'small' depending on the application domain"

Examples given include a civil aircraft failing during a typical eight hour flight and a high speed network node experiencing a buffer overflow. Another introductory text on rare event simulation [11] does not propose a definition, concentrating instead on the randomness and uncertainty of rare events.

It should be noted that the term 'rare event' is uncommon in WSN research, the literature containing only a handful of papers with the words 'rare' and 'event' in their title $[12,13,14,15,16,17]$, of which only four have been cited by others ${ }^{1}$ $[14,15,16,17]$. However, this thesis embraces existing research that contains techniques, algorithms and analysis that may prove useful in WSNs deployed to detect rarely occurring events.

Rare event WSNs are found in a variety of situations including the battlefield [18] where low unit costs allow high density, short time-frame, disposable deployments. In industrial settings [19] WSNs are cost efficient when compared to fixed wiring, where robust self-organizing characteristics make them suitable for monitoring hazardous machinery and protecting high value assets.

It is entirely practical to perform periodic data collection and rare event detection in one WSN, but quality of service (QoS) aware routing protocols [20] should be implemented to prevent unacceptable delays when urgent message relating to rare events have to queue up behind more mundane traffic. Whatever the deployment scenario, two metrics emerge as fundamental to rare event sensing $[17,21,22]$ :

\footnotetext{
${ }^{1}$ As reported by Google Scholar, August 19, 2015
} 
Detection Probability Likelihood of an event being detected.

Detection Delay Time taken for event notification to reach a network sink.

WSNs have been classified by their data delivery profile as being continuous, event-driven, observer-initiated (query-based), or a hybrid [23], the latter two falling outside the scope of this thesis. In continuous sensing scenarios data samples are taken periodically; between samples the transceiver or the entire node can be powered down. Increasing the gap between samples leads to an increase in network life. Event sensing, with the assumption that events occur randomly, introduces additional complexity as a node that is not energised cannot sense the event and a node with a powered down transceiver can take no part in forwarding notification messages.

Critical events that are both ephemeral and transitory pose a further challenge to WSNs deployed for their detection. Maximising detection probability and minimising detection delay requires sufficient nodes are active at all times to both maintain initial sensing coverage and provide a route to the network sink delayed only by transmission overhead. Energy saving pauses during transmission such as those implemented in media access control (MAC) protocols featuring receiver initiated transceiver de-activation [24] or demand wake-up [25] could be regarded as having too great an impact on detection delay. For the algorithms presented in this thesis, detection delay is minimised by having an always on, always connected communication route to the network sink at the cost of significant energy consumption through idle listening.

Establishing and maintaining sensing coverage in WSNs for rare events can be regarded as ensuring every point in the sensing area is covered by at least one sensor node at all times whilst maintaining energy efficiency [26]. WSNs with a sufficiently dense overpopulation of sensing nodes can self-organise such that a given node is in a position to power down if a subset of its neighbours are willing to take temporary responsibility for its sensing area. In battery powered WSNs, this duty-cycling extends the operational lifetime of a subset of deployed nodes and in doing so can both extend the period during which the network maintains its initial coverage and the period during which there is at least some coverage [27].

When energy harvesting replaces battery power, duty-cycled nodes not only preserve their stored energy but also have the opportunity to replenish it more rapidly than when active. The design of solar energy harvesting systems for 
WSNs is non-trivial [28], yet if the energy harvested and stored is sufficient, networks have the opportunity to maintain initial sensing coverage for extended periods with the potential for indefinite operation. For solar energy harvesting, the physical dimensions, output voltage, maximum current and efficiency of installed solar panels determine the harvestable energy for a given incident radiation. Once harvested, energy is lost through inefficiencies in storage componentry yet recent low-power management systems demonstrate charging efficiencies in excess of $90 \%$ [29].

It can be convenient to assume Internet of Things (IoT) [30] based sensor networks [31, 32] in, for example, smart cities [33] are permanently energised; in practice, mains power may not be available at all times, most notably during rare extreme weather events. Vortex shedding [34] caused by high winds can damage highway light columns [35]; if sensors are to be deployed to detect the significant displacements experienced during such an event [36] as shown in Figure 1.1 they would need to remain energised even when the light column looses power. Energy harvesting provides a mechanism for such networks to maintain independence from the power grid. In this circumstance, extremely small scale harvesting could be deployed as energy would be available for harvesting from the pole itself either from the mains power line [37] or by scavenging artificial solar radiation when the light pole is illuminated.

Energy harvesting promises to provide extended life to WSNs and has been extensively investigated for data capture applications [38], but comparatively little research exists on the successful application of this technology to rare event sensing.

\subsection{Synopsis}

The research presented here is motivated by the desire to guarantee detection and rapid propagation of notification messages in WSNs targeting rarely occurring events of critical importance. Assuming an operational life measured in years rather than weeks is required, network owners can deploy sensing devices with large scale energy harvesting capability. However, this may prove inappropriate for aesthetic reasons or space limitations, in which case inexpensive small devices with small scale energy harvesting capabilities become desirable. In this situation, duty cycling energy harvesting networks are necessary as they have 


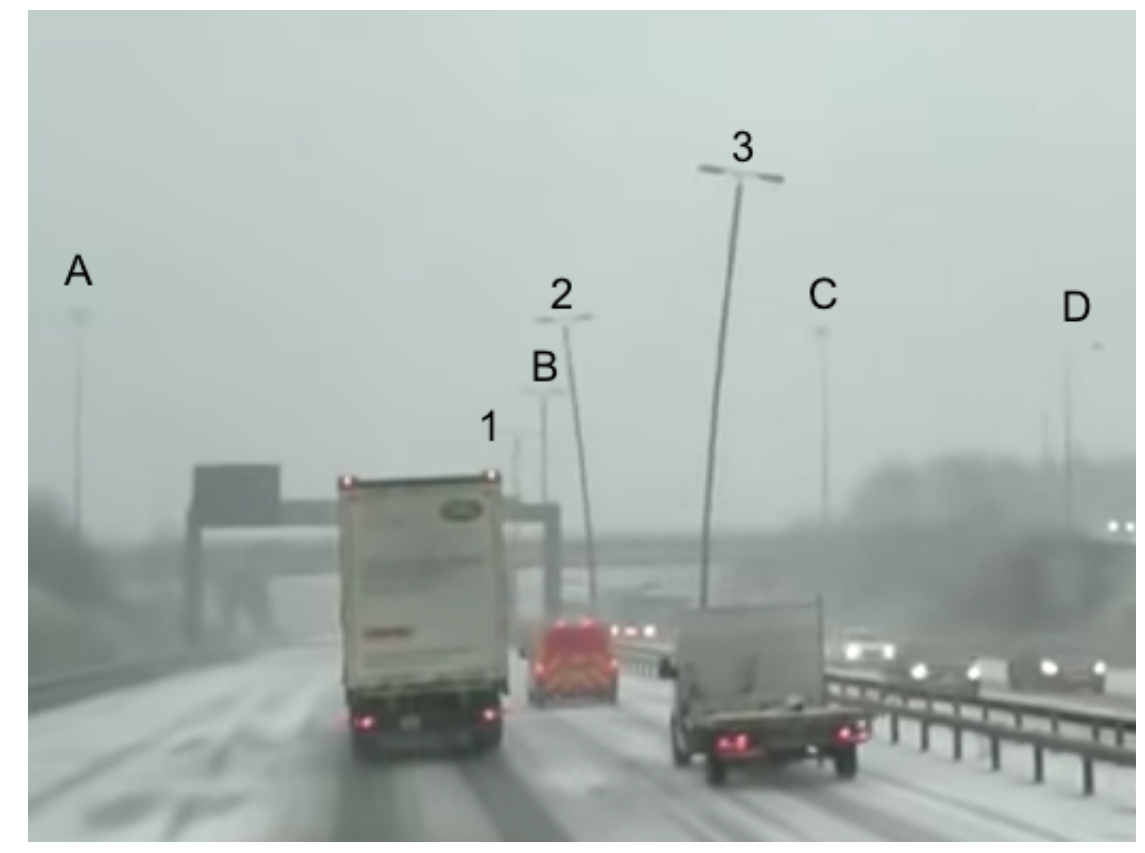

Figure 1.1: Light poles on the M62 in England; still from [36]. Poles 1, 2, \& 3 are vortex shedding, A, B, C, \& D are not. The video clip author suggests pole B is unaffected as it is immediately behind the gantry. Note mains power appears to have been lost as none of the lamps or gantry signs are illuminated.

the potential to provide indefinite operation even for devices with modest capabilities.

Challenges facing these small devices include considerations of stored charge equalisation amongst harvesting nodes, prioritisation of forwarding speed over other issues including energy consumption, provision of firm delivery guarantees, ensuring as many nodes as possible are asleep at any given time, and practical aspects of constructing an algorithm to satisfy all these challenges. Each challenge is briefly described below.

Energy Equivalence If coverage is to be maintained along with a path to forward notification messages, allowing nodes to exhaust their energy supplies in an uncoordinated fashion can lead to some nodes becoming inactive whilst others remain energised.

Rapid Forwarding For infrequent events forwarding decisions take priority over other considerations including energy consumption. Even the least energy efficient forwarding algorithm has little impact on long-term energy consumption as event notifications rarely need to be transmitted. 
Guaranteed Delivery Rapidity of message forwarding must not be gained at the expense of guaranteed delivery.

Sleep Maximisation Significant over population of a sensing area can be advantageous as more nodes are simultaneously in a position to sleep than in less dense deployments.

Combined Algorithm Maximising network lifetime and ensuring swift as possible forwarding have previously been seen a fundamentally conflicting objectives. Even when both are achieved separately combining them is problematic if one is not to gain at the expense of the other.

\subsection{Contributions}

This thesis explores and makes contributions in multiple research areas, including the accuracy of energy models used for WSN research, sleep equivalency as the basis for duty-cycling algorithms, concentration on rapidity of message propagation as a primary concern in geographic forwarding algorithms, and the successful combination of coverage and connectivity concerns into as single, unified algorithm. In detail, the contributions made by this thesis are that:

1. Critical rare event sensing is a research area distinct from previously well studied related topics.

2. Invalid assumptions made about energy consumption of real devices can lead to the construction of inaccurate energy models for finite state simulations. Of particular note is the potential to invalidate all network forwarding schemes based on the assumption that reducing the transmit power of a low-power radio transceiver causes it to consume less energy when transmitting packets.

3. Ensuring sleep or stored charge equivalence in coverage preserving duty cycling schemes maximises the operational life of battery powered WSNs and makes effectively indefinite operation a realistic goal when energy harvesting is adopted.

4. Ignoring traditional concerns including collision avoidance and energy conservation in geographical forwarding algorithms running on standards based 
MAC protocols allows maximally rapid propagation of event detection messages.

5. In forwarding algorithms that do not attempt to avoid collisions, it is shown that information fusion techniques utilising time and geometry based heuristics to suppress duplicate event notification messages are of little practical benefit.

6. Maximum detection probability and minimal detection delay can be realised in a single protocol that preserves sensing coverage and ensures a permanently connected network.

\subsection{Outline}

This thesis progresses from investigatory research to presentation of a novel algorithm with the ability to maintain perfect sensing coverage and network connectivity to deliver rapid forwarding of critical event notifications.

Chapter 2 Surveys related work in three areas: (1) Strategies most frequently used in event sensing networks. (2) Existing coverage and connectivity algorithms with a focus on their ability to maximise detection probability and/or minimise detection delay. (3) Location aware geographic forwarding algorithms and how they approach minimising detection delay.

Chapter 3 Experimentally demonstrates that a number of commonly held beliefs about how small-scale WSN devices consume power are invalid. This insight is used to construct an accurate energy consumption model for simulations undertaken in subsequent chapters.

Chapter 4 Introduces ESCARGO, a coverage preserving collaborative dutycycling algorithm for energy harvesting sensor networks focusing on equalisation of power consumption over time.

Chapter 5 Describes SOFIE, an opportunistic algorithm for non-deterministic rapid forwarding of critical event detection messages through sensor networks with rapidly changing topologies.

Chapter 6 Combines guaranteed coverage and rapid forwarding into a single algorithm, CPRF. Favourable comparisons are made to previously proposed 
schemes and a physical implementation is trialed in live outdoor deployments on commercially available low-power sensing devices.

Chapter 7 Summarises the research presented and identifies areas for further investigation. 


\section{Chapter 2}

\section{Related Work}

WSNs can be considered part of the Internet of Things (IoT) [30] and continue to benefit from active research projects and regular publication of surveys targeting specific topics. Recently, statistical, probabilistic, artificial intelligence, and machine learning methods for event detection have been briefly surveyed [39] yet the majority of WSN surveys in the last five years have focused on areas unrelated to rare events. One exception [40] does consider event sensing as it relates to energy efficient routing with an emphasis on how the criticality of an event informs protocol design. Table 2.1 lists ten of the most frequently cited WSN survey papers published since January 1, 2010 with their area of interest.

Few algorithms address both sensing coverage and explicit maintenance of network topology to facilitate forwarding and/or routing; one that does is $\mathrm{A} 3 \mathrm{Cov}$ [41], a topology construction algorithm with a non-localized and inexpensive way of producing a connected backbone. Once a connected topology is established, $A 3 C o v$ adds extra nodes to provide a partial coverage solution. A3Cov is favourably compared to an area-based collaborative sleeping protocol for WSNs (ACOS) [42], yet it does not attempt to provide perfect area coverage, rendering it unsuitable for critical event sensing. Further, the $A 3 \mathrm{Cov}$ algorithm introduces cases in which a node may choose to sleep despite doing so compromising connectivity in a section of the network. A timeout based second opportunity process is required to mitigate this problem but does not remove it entirely, adversely impacting notification delay.

The literature suggests it is more usual to consider sensing coverage and forwarding as separate issues. Section 2.1 enumerates common strategies for rare event sensing and surveys existing research including energy harvesting initia- 
tives. Section 2.2 details related work on coverage preservation, while Section 2.3 examines research on forwarding techniques.

Table 2.1: Most Cited WSN Surveys Since January 2010

\begin{aligned} \hline Survey & Area of Interest \\ \hline$[43] &$ Localisation \\ ${[44] } &$ Simulators \\ ${[45] } &$ Key management \\ ${[46] } &$ Heterogeneous clustering algorithms \\ ${[47] } &$ Security \\ ${[48] } &$ IP based WSNs \\ ${[49] } &$ Sensing coverage \\ ${[50] } &$ Industrial automation \\ ${[51] } &$ Security \\ ${[52] } &$ Security \end{aligned}

\subsection{Event Sensing Strategies}

This thesis is interested in WSN research that either specifically targets rare event sensing, considers work on rare events to be related, or documents ways the presented work could be applied to rare event sensing. Issues generic to all WSNs, including but not limited to security [53], software distribution [54], and radio interference [55] are deemed out of scope. Similarly, event sensing techniques unrelated to the rarity of the event in question are not considered relevant. Table 2.2 illustrates the relative volume of surveyed work for the following event sensing strategies:

Collaboration In large multi-hop sensing networks, the energy cost of long-distance communication is significant. If sensing nodes work locally to collaboratively remove false positive event detections, network lifetime can be extended without adversely impacting detection probability. Collaboration can also take the form of nodes with discrete capabilities working together to more reliably detect rare events.

Duty Cycling Shutting the entire sensing node down periodically is the ultimate energy saving technique. When nodes synchronise their sleep sched- 
ules, predictable levels of detection probability and delay can be maintained whilst extending the life of the network.

Component Deactivation It is not always necessary for all WSN node components need to be constantly energised. Deactivating power hungry devices, particularly radio transceivers, saves energy and leads to longer network life-spans, extending the period in which a high detection probability can be maintained.

Over Population Densely deploying sensing nodes such that they introduce redundancy in the network enables local collaboration and synchronised duty cycling in addition to an increased tolerance to individual node failure.

Message Suppression When a number of sensing nodes detect the same rare event, their detection messages may be regarded as duplicates of each other. Suppressing these duplicates reduces network traffic and saves energy in doing so.

Burst Aware Protocols When a rare event is detected by multiple sensors, a burst of network traffic is generated calling for protocols designed to handle the resulting media contention without introducing unnecessary detection delay.

Always On Whilst not suitable for all deployment scenarios, the simplest strategy for maximising detection probability and minimising detection delay is to permanently energise all nodes in the network.

Energy Harvesting The challenge of maximising network longevity and detection probability, and minimising detection delay can be moderated by harvesting environmental energy to augment or replace batteries in sensing nodes.

Diversity in the characteristics of rare events prevents any one sensing strategy from becoming a panacea. Further, even given a well defined event classification, utilizing a single strategy may be insufficient to minimise occurrences of undetected events whilst maximizing the operational life of the sensing network. This section introduces event sensing strategies with examples of existing work examining them and surveys to what extent each strategy is represented in the literature. 
Table 2.2: Volume of Surveyed WSN Research on Rare Events

\begin{tabular}{lc}
\hline Sensing Strategy & Papers Surveyed \\
\hline Collaboration & 17 \\
Duty Cycling & 15 \\
Component Deactivation & 14 \\
Over Population & 8 \\
Message Suppression & 7 \\
Burst Aware Protocols & 7 \\
Always On & 7 \\
Energy Harvesting & 6
\end{tabular}

Table 2.3 summarises surveyed event sensing strategies, indicating their typical impact on detection probability and detection delay. The following subsections describe each strategy in more detail. 


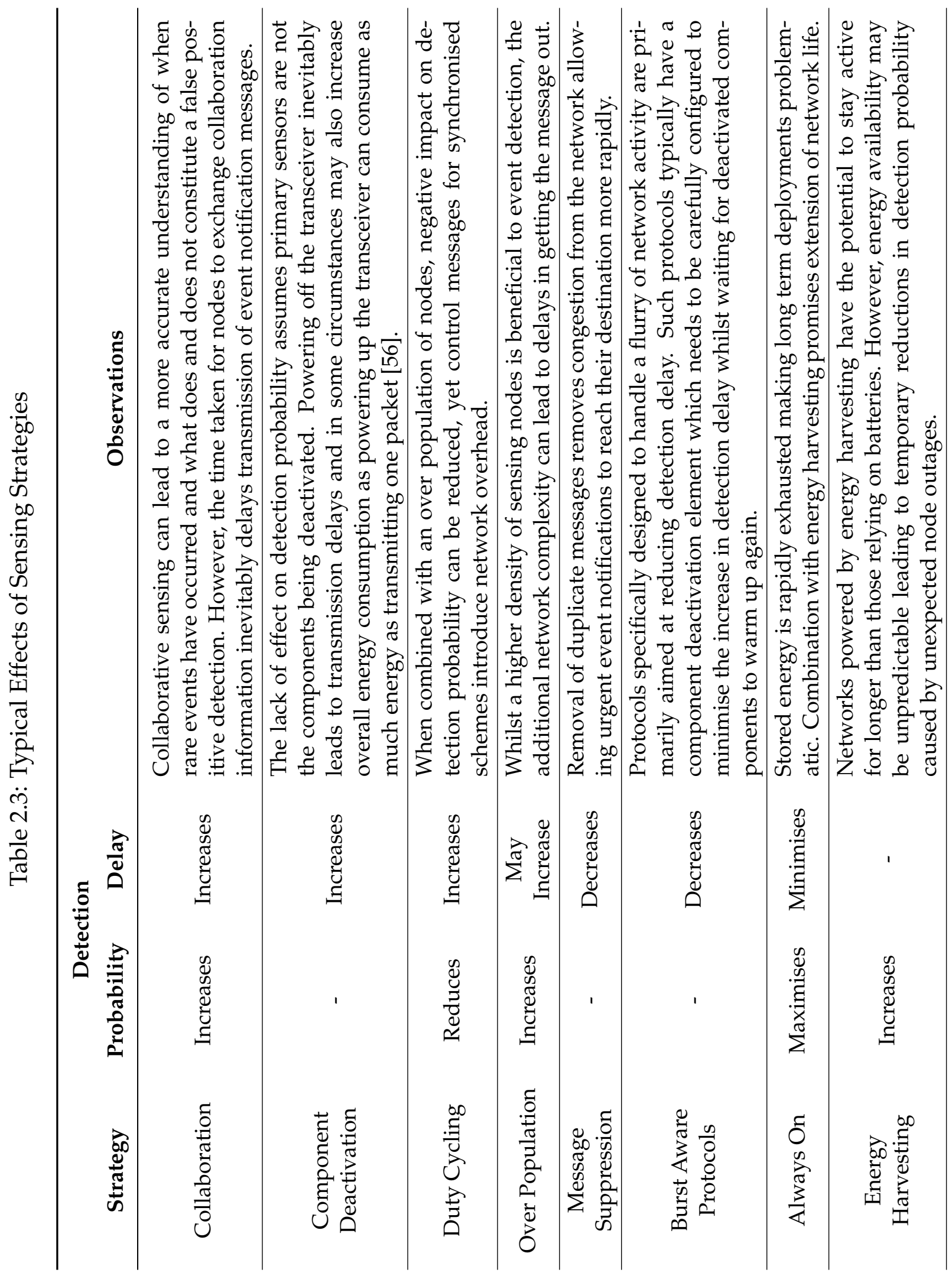




\subsubsection{Collaboration}

In large multi-hop networks, the cost of propagating event detection messages from originating nodes to the base station can be significant. If adjacent nodes collaborate prior to initiating the expensive long distance communication, a group decision is made on whether or not sufficient collective sensing evidence exists to support publication of an event detection message. A survey of cooperative event detection algorithms [57] claims distributed evaluation exhibits the best energy efficiency and highest detection accuracy when compared to local and centralised approaches.

In a recently proposed probabilistic event monitoring scheme (PEMS) for sparse networks [58], sensing nodes detecting an event collaborate to select those whose observations are probabilistically significant. Nodes collaboratively selected for event monitoring then adjust the network topology for improved energy efficiency by assigning responsibility for data forwarding to a single clusterhead.

A system capable of being trained to recognise application specific event types [59] has been field-tested for construction site intrusion detection with 100 nodes. Transmitting sensed data to a base station for analysis is eschewed in favour of collaboration between sensing nodes to determine if an event has occurred. Each node has multiple sensors and extracts feature vectors from raw data. Adjacent nodes share their feature vectors; if and only if they aggregate to match trained or statistically defined event vectors will a detection message be published.

Rather than a simple threshold breach, more sophisticated event signatures can be used for distributed detection of transient complex events. In [60] nodes are assumed to be stationary, location aware and uniformly distributed, with a path existing between each pair of nodes. The sensor network is partitioned into equal size cells. Nodes within a cell rotate leadership responsibilities including intra-cell communication where a time stamped weighted average value for the cell is shared with adjacent cells. For a network represented by $p \times q$ cells, an event signature is an $r \times s$ matrix where $r<p$ and $s<q$. To detect an event, leadership nodes "overlay" the event signature on the nodes in their cell; if a match is found, the event is considered to have been detected and an appropriate message is forwarded to the base station.

Distributed Bayesian algorithms for detection of sensing faults in WSNs tasked with the binary detection of environmental events [61] have been shown through theoretical analysis and simulation to correct 85-96 percent of faults even when as 
many as 10 percent of the nodes are faulty. [62] present related simulation work whilst also evaluating Neyman-Pearson approaches and more closely considering the energy efficiency of their algorithms.

[63] identify that clustering nodes and assigning a clusterhead that takes responsibility for forwarding detection messages from the cluster to a base station may save energy. The authors propose a distributed, randomised clustering algorithm and extend it to generate a hierarchy of clusterheads. Energy efficiency is observed to improve as the number of levels in the hierarchy increase.

Real-time event detection services are provided in Data Service Middleware (DSWare) [64] by collaborative correlation of sensing observations based on event characteristics enabling in-network differentiation between event occurrences and false alarms. Data semantic based confidence functions are supported to determine the relative importance of sub-events and capture historical patterns. If detection rates are low, partial detections of critical events are reported.

Military field tests of a distributed target classification system based on collaborative signal processing [65] proved successful. Objects in the sensor field generate time varying spatial signatures from multiple sensors, and a moving object is a peak in the signature field that moves over time. Tracking the object is equivalent to tracking the peak. Distributed tracking is achieved by dividing the sensor field into cells within which a node is designated the manager, responsible for inter-cell coordination and intra-cell communication. In collaborative detection, classification, and tracking of moving targets: (1) cells near potential target trajectories are alerted and nodes in these cells collaborate to sense a target; (2) when a target is detected, the cell becomes active and tracking is initiated if the target is of the required type; (3) estimates of the target's current location and velocity are used to estimate potential future locations of the target; (4) as the target approaches the cell boundary, cells on the estimated trajectory are alerted and the process repeats.

The Role Alternating Coverage Preserving Coordinated Sleep Algorithm (RACP) [27] and the Equitable Sleep Coverage Algorithm for Rare Geospatial Occurrences (ESCARGO) [1] collaboratively modify the duty-cycle (Section 2.1.2) of an over population (Section 2.1.4) of sensing nodes to reduce network-wide energy consumption whilst maintaining sensing coverage.

Energy efficiency through energy awareness of cooperating sensor nodes is the focus of another surveillance system [66] aimed at military deployments. Trade offs between energy and surveillance efficiency are made by adjusting the 
sensitivity of the system. On initialization, nodes discover their neighbours and elect sentry nodes who monitor the environment for intruders, while the remaining nodes enter a low power mode. If an intrusion is detected by a sentry, it wakes up the other nodes and they collaborate to track the intruder. When the intruder leaves the sensing area, the system resets, alternate sentries are selected, and the process continues.

Collaborative fuzzy logic schemes for event detection have been proposed $[67,68,69]$ and surveyed [70] though specific applications to rare events have yet to be documented. One challenge fuzzy logic algorithms face is that they typically require large rule-bases, the storage and distribution of which can prove problematic for the limited resource devices typically used in WSNs. Less specific machine learning techniques have recently been proposed for collaborative detection of definitively rare events, namely leaks in water distribution pipelines [71].

Works detailed in other sections of this section that also feature collaborative event sensing are: $[72,73,74,18,5]$.

\subsubsection{Duty Cycling}

In many event sensing scenarios, an always on strategy (Section 2.1.7) fails to meet longevity requirements beyond a few days or weeks. For extended use, regularly powering nodes down (putting them into sleep mode) improves operational life, potentially at the expense of guaranteed event detection.

Maximizing the detection probability for transient events and minimizing the detection delay for persistent events are fundamental to acceptance of duty cycling as an appropriate strategy for event-driven WSNs. [75] characterise the trade-off between system lifetime and detection performance; an algorithm to collaboratively determine when nodes should wake up is proposed and a favourable comparison is made to random sleep scheduling.

Recent research [12] specifically targeting rare events proposes a probabilistic duty cycle in sensor medium access control (PDC-SMAC) algorithm for infrequent events in a military scenario intended to reduce the energy cost of 'ineffective sensing' - periods where a node is energised but there is nothing to sense. Sleep scheduling [76] promises bounded detection delay while maximizing network lifetime. [17] introduce a scheme where nodes enter and exit sleep mode in a coordinated fashion ensuring sensing coverage rotates over the network, each 
node being energised at regular intervals. For persistent events, this scheme introduces an increase in detection delay, with a reduction in detection probability being inevitable for transient events. In scenarios where network longevity takes priority, schemes of this nature may be acceptable.

For situations where the WSN is responsible for alerting humans in the sensing area, such as forestry workers when a fire breaks out or miners when a gas leak is detected, detection delay is considered the critical factor as the complex multi-hop nature of disseminating information through a large network can be adversely impacted by intermediary nodes adopting sub-optimal duty cycles. A level-by-level offset schedule based duty cycle pattern [77] has been proposed that restricts the upper bound of detection delay to be $3 D+2 L$ where $D$ is the hop count to a central node and $L$ is the duty cycle sleep time. The scheme proposed in [77] adopts a two phase alarm broadcast following detection of the rare (the authors use the term 'critical') event. In the initial phase an event event notification is sent to a central node which initiates the second phase where the alarm is broadcast to all other nodes.

Adaptive control of the duty cycle [78] for energy harvesting WSNs (Section 2.1.8) allows sensing nodes to respond to changes in the environment. Such schemes have been shown to perform better than techniques that require a priori knowledge of available energy. This combination of techniques typifies successful rare event sensing research where two or more approaches compliment each other. Duty cycling and energy harvesting are individually advantageous techniques that when combined provide even greater benefit.

Full node duty cycling is also considered in the following papers detailed in other sections of this section: [79, 80, 81, 66, 27, 82, 83]

\subsubsection{Component Deactivation}

An alternative to full node duty cycling (Section 2.1.2) is powering down components when they are not required. The component of WSN nodes that uses the most energy is the wireless transceiver (Table 2.4). Transmit and receive costs are inevitable, but energy is wasted by media collisions and associated retries. Idle listening, where the transceiver is powered up but receives no packets, is another significant waste of energy. Deactivation of components can be application controlled or initiated by other layers, most notably MAC where the transceiver is often allowed to sleep when there is nothing for it to listen for or receive. 
AIMRP, an Address-light Integrated MAC and Routing Protocol [84] is specifically aimed at rare event detection scenarios. A randomised power saving mode allows nodes to de-activate their transceivers independently of one another. Using simulations, AIMRP has been shown to compare favourably to the more generic sensor MAC (S-MAC) [85] for event detection applications. S-MAC also supports transceiver deactivation, virtual clusters of nodes perform localised sleep synchronization. Energy consumed by AIMRP and S-MAC are compared for selected values of $\tau$, the maximum permissible end-to-end latency for event reports, $\tau$ being equivalent to the maximum allowable detection delay; Simulation and analysis of AIMRP shows it consumes less energy than an analysis of S-MAC suggests it would for values of $\tau$ between $0.015 \mathrm{~s}$ and $0.05 \mathrm{~s}$.

At the application level, a military intrusion system "Line in the sand" (LITS) [18] makes the distinction between passive and active sensing. Passive sensors are ones that measure analogue properties of the intruder such as magnetic, thermal and acoustic characteristics, while active sensors are those that determine the intruders' range, velocity and direction of travel by how the target modifies, reflects or scatters a signal transmitted by the sensor. The active sensor in LITS is pulse Doppler, while a magnetometer is used as a passive sensor. Detection of an intruder by the cooperatively low powered passive sensor triggers initialization of the active sensors. In LITS the active and passive sensors are on different nodes which collaborate, with the passive nodes detecting the intrusion and the active nodes tracking it.

A source-initiated or sink-initiate wakeup radio (WUR) based medium access control (GWR-MAC) [86] aims to reduce idle listening and by doing so improve energy efficiency in short range communication networks such as wireless sensor and body area networks. Favourable analytical comparisons are made against conventional duty-cycled (Section 2.1.2) MAC Protocols suggesting GWR-MAC would be useful and energy efficient for sensing low frequency events requiring a reasonably low detection delay.

The TRafficAadaptive Medium Access protocol (TRAMA) [87] is a time slotted MAC protocol with a distributed election scheme. TRAMA guarantees collision free transmission and allows nodes to power down their transceivers when they are not transmitting or required to receive. Additional MAC protocols that deactivate the transceiver for short periods are described in Section 2.1.6.

Messages used to control a sensing network are typically modestly sized and sent in small batches at high frequency; those sent in response to event detection 
tend to be much larger, sent in higher volumes but far less frequently. Deploying two radios on each sensing node [88], a low power device for control traffic and a higher power component for event data, allows the more energy hungry device to be powered down almost all the time.

Component deactivation is also considered in the following papers detailed elsewhere in this section: $[89,25,24,16,66,90,5,91]$

Table 2.4: Current Draw for WSN Components

\begin{tabular}{lcc}
\hline & Active & Sleep \\
\hline Texas Instruments CC2420 Transceiver $^{1}$ & $18.8 \mathrm{~mA}$ & $1 \mu \mathrm{A}$ \\
Texas Instruments MSP430 Microcontroller $^{1}$ & $1.8 \mathrm{~mA}$ & $5.1 \mu \mathrm{A}$ \\
Analog Devices ADXL345 3-Axis Accelerometer $^{2}$ & $40 \mu \mathrm{A}$ & $0.1 \mu \mathrm{A}$ \\
Panasonic EKMB123112 Passive Infrared Sensor $^{3}$ & $2 \mu \mathrm{A}$ & - \\
\hline${ }^{1}$ From www.memsic.com & & \\
${ }^{2}$ From www.analog.com & & \\
${ }^{3}$ From pewa.panasonic.com & &
\end{tabular}

\subsubsection{Over Population / Node Redundancy}

In a network with sufficient sensing coverage when all nodes are energised, duty cycling (Section 2.1.2) increases network longevity, but reduces coverage. Over populating the sensing area allows nodes to synchronise their duty cycle so sensing coverage is maintained when some subset of nodes are powered down. Installing redundant nodes where each primary sensing node has a partner in close proximity allows optimal coverage to be maintained and can provide some tolerance to faults.

SenSlide [92], a landslide prediction system, is a WSN hybrid of event detection and data capture. Energy-aware routing protocols are utilised to avoid individual nodes becoming drained of power prematurely but a level of fault tolerance is principally achieved by deploying redundant nodes.

For ephemeral events where a less than perfect detection probability is unacceptable, duty cycling algorithms can preserve coverage when an over population of nodes is deployed. A coverage preserving role alternating algorithm [27] enables such a deployment to extend the period during which initial sensing 
coverage is preserved by allowing nodes to enter sleep state if a minimal subset of neighbouring nodes have agreed to collaboratively take responsibility for the sleeping node's sensing area. When the sleeping node wakes up, sponsoring neighbours are now free to enter sleep mode if they can agree on a sponsoring contract with a group of their own neighbours.

Other works surveyed that rely on, or consider an over population of sensing nodes are: $[1,75,80,17,82,18,83]$

\subsubsection{Message Suppression and Data Aggregation}

When a rare event is sensed by multiple nodes in close physical proximity, they generate messages that can be considered duplicates of one another; delivery of a subset of these messages may be sufficient to confirm event occurrence [93,94].

CC-MAC [95], composed of an event MAC (E-MAC) and a network MAC (N$\mathrm{MAC})$, exploits the spatial correlation of messages generated by sensing nodes to suppress duplicates from nodes in close physical proximity. In a given correlation region, a single node is chosen as being representative of its correlation neighbours; only messages originating at the representative node are transmitted to the network sink, all other messages are suppressed.

Sift [96] is a slotted MAC protocol designed to deal with both the bursty nature of a large number of nodes wishing to transmit at the same time (Section 2.1.6) and de-duplication of the message queue. In removing some percentage of duplicate messages, Sift aims for the collision free delivery of $R$ messages from $N$ nodes where $R<N$. On successful delivery of $R$ messages, the remaining $N-R$ messages are suppressed.

Message aggregation techniques for data-centric routing [97] include suppression of duplicates to reduce energy usage by minimising transmission volumes. In dense networks, greedy aggregation has been shown [98] to be more efficient than opportunistic aggregation schemes. Sliding window skylines [99] have been also proposed as a suitable technique for message suppression in a system for forest fire detection [100] .

\subsubsection{Burst Aware Protocols}

Rare events can trigger a tsunami of messages in the sensor network, all of which would like to be transmitted at the same time leading to channel contention, an 
increased probability of packet collision and the potential for data loss and delay.

The MAC layer defined by the IEEE 802.15.4 standard [101] and used extensively in WSN devices has two modes of operation. In beaconed mode, at least one device acts as a personal area network (PAN) coordinator and noncoordinator devices must wait to transmit in contention free time slots. If beaconing is not employed, an un-slotted carrier sense multiple access with collision avoidance (CSMA/CA) algorithm based on listening to the physical medium is used, collisions being avoided by invoking a random exponential back-off algorithm. Beaconed or not, IEEE 802.15.4 MAC is designed to minimise energy usage in the nodes, not maximise delivery rates. In extremely bursty conditions, an alternate MAC may be required.

A geographical cross-layer asynchronous sender-oriented MAC protocol [102] has recently been proposed to facilitate opportunistic routing in low duty-cycle wireless sensor networks (Section 2.1.2) that may experience bursty traffic patterns and unpredictable changes in network topology. This protocols objective is to maximize network lifespan while guaranteeing packet delivery with acceptable end-to-end delays.

Reliable Bursty Convergecast (RBC) [103] is a MAC protocol designed specifically to handle bursts of data in multi-hop networks that converge on a limited number of sinks. Using data traces taken from [18] an implementation of RBC based on B-MAC [90] is shown experimentally to perform better than the default TinyOS [104] radio stack where $100 \%$ packet delivery is not required.

S-MAC [5] is an earlier MAC protocol for ad-hoc deployments of battery operated sensor nodes that remain inactive for extended periods but become suddenly active when an event is detected. S-MAC values energy conservation and self configuration over per-node fairness and latency. Virtual clusters of nodes adopting common sleep schedules reduce control overhead and message passing techniques can reduce contention latency for applications undertaking innetwork data processing.

A Receiver-Initiated asynchronous duty cycle MAC protocol for dynamic traffic loads (RI-MAC) [24] is a transceiver deactivation (Section 2.1.3) protocol shown to outperform contemporaries when faced with bursty message flows. Dutycycle Scheduling based on Residual energy (DSP) and Duty-cycle Scheduling based on Prospective increase in residual energy (DSR) [89] are protocols derived from RI-MAC that determine their deactivation schedules based on residual or prospective increases in harvested energy (Section 2.1.8). 
A MAC protocol designed to cope with the burst of traffic that follows detection of a rare event is Demand Wakeup MAC (DW-MAC) [25]; sleeping transceivers are woken on demand yet data transmissions are guaranteed not to collide at receiving nodes. DW-MAC is a synchronised duty cycle MAC protocol that assumes accurate clock synchronisation by. Each cycle is divided into three periods: Sync, Data and Sleep. DW-MAC wakes up nodes on demand during the Sleep period of a cycle in order to transmit or receive a packet.

Sift [96] is a MAC specifically for event sensing networks, its principles being similar to those found in rare event WSNs powered by energy harvested from the event itself [105] where bursts of data must be dealt with before the limited available energy is consumed.

\subsubsection{Always On}

Leaving event sensing nodes powered on at all times may be necessary if missing an event occurrence is unacceptable, notification of the event needs to be as fast as possible or the network deployment timescale is sufficiently short that battery depletion is unlikely. Estimating the longevity of always on sensing networks is problematic. In a study of a large intrusion detection system [106] it is suggested that mistakes as large as an order of magnitude are routinely made. Nevertheless, adopting an always on strategy remains an appropriate course of action in certain circumstances.

Structural engineers monitoring vibrations require rich data at high sample rates yet bandwidth limitations in WSNs made up of more than a handful of nodes make reliable delivery of such a data deluge impractical. Wisden [107] observes that a single triple axis accelerometer generating 16-bit samples at $100 \mathrm{~Hz}$ requires $4.8 \mathrm{Kbps}$ and suggests delivery of data relating to interesting events (rather than a continuous time series) is sufficient for analysis of structural vibration. Even taking this into consideration and allowing for data compression before transmission, in such an environment sensor nodes are busy almost all the time.

Monitoring events on an active volcano with small low-power WSN devices [108] was achieved by leaving the sensing nodes energised at all times. The short deployment (three weeks), volume of events detected (230), their ephemerality (less than 60 seconds each) and transient nature made an always on strategy acceptable. Similarly, a system for surveillance of temporary museum exhibits [109] stays energised at all times to maximise detection probability; extending network 
life is of little concern as batteries can provide sufficient power for an entire exhibition.

Permanently powering all WSN nodes is a technique adopted in a military intrusion detection system [18] and is referenced when considering coverage maintenance in low duty cycled WSNs [27].

\subsubsection{Energy Harvesting}

WSN research has traditionally focused on minimizing energy consumption in battery powered sensor nodes, balancing longevity against functionality. Increasingly, researchers are evaluating the efficacy of harvesting energy from a variety of environmental sources $[110,111]$.

Energy sources such as solar are reliable (the sun always comes out) but unpredictable (it may be obscured by clouds). For rare event sensing where the event may occur at a point where limited environmental energy is available, a Harvest-Store-Use architecture [111] coupled with multiple storage devices as used in Prometheus [112] and AmbiMax [113] would appear appropriate, yet neither Prometheus nor AmbiMax address rare event sensing directly, Prometheus being specifically aimed at periodic data capture.

A proposed adaptive scheduling scheme for cooperative energy harvesting networks [114] combines cooperative communications (Section 2.1.1) and energy harvesting in a scheduling scheme intended to maximizes packet delivery ratio with the aim of gaining acceptable detection probability without necessarily minimising detection delay. Cooperating nodes advertise their status as either 'active/on' or 'inactive/off' (a form of duty cycling (Section 2.1.2) but without component deactivation (Section 2.1.3)) based on their harvested energy levels. When a node detects an event and wishes to transmit an notification message, if a cooperating node is currently marked as 'active', the source node will forward the message via it's cooperating neighbour; otherwise the source nodes attempts to transmit on its own. Simulation results indicate the scheme would provide similar performance to a state-of-the-art alternative [115], but does not require threshold parameter optimization.

An adaptive scheme for duty cycling (Section 2.1.2) [78] in energy harvesting WSNs does not attempt to model the energy source allowing usage in situations where a prior knowledge of the source is unavailable. The scheme's computational efficiency and adaptability to near depletion scenarios prevent node 
outages whilst tunable stability restricts variance in individual node duty cycles. Similarly, ODMAC [116], an on-demand MAC specifically aimed at energy harvesting WSNs supports individual duty-cycles for nodes with different energy profiles but does not consider its application to rare event sensing.

DSR and DSP [89] (cf: Section 2.1.6) propose dynamic duty cycle scheduling for energy harvesting WSNs based on RI-MAC [24]. DSR allows sensor nodes to adjust their duty cycle based on their remaining energy store. DSP estimates future energy harvesting opportunities and aggressively adjusts duty cycles proportionately. Reductions in end-to-end delay are demonstrated for DSR and DSP over unmodified RI-MAC for $N \times N$ grids, when $N$ is in the range $4 m$ to $8 m$, with $20 \mathrm{~m}$ between each node and a transmission range of $30 \mathrm{~m}$.

A survey of energy harvesting for structural health monitoring (SHM) sensor networks [117] did not restrict itself to rare events but recent research [105, 118] addresses SHM via WSNs powered by energy extracted from the rare event itself, namely an earthquake. The focus is on a MAC protocol that is capable of handling both the bursty nature of the messages generated by the event (Section 2.1.6) and the limited energy extracted from the earthquake via frequency tuned piezoelectric vibration energy harvesters.

A detailed survey of power management in energy harvesting WSNs [119] considers event monitoring as a deployment example. Particular attention is paid to detection delay making a distinction between the delay introduced by the MAC layer and energy aware routing protocols.

\subsubsection{Classification of Existing Work}

Table 2.5 summarises work discussed in this section highlighting which sensing strategies are featured in deployed applications, MAC protocols and supporting technologies. 
Table 2.5: WSN Research on Rare Event Sensing

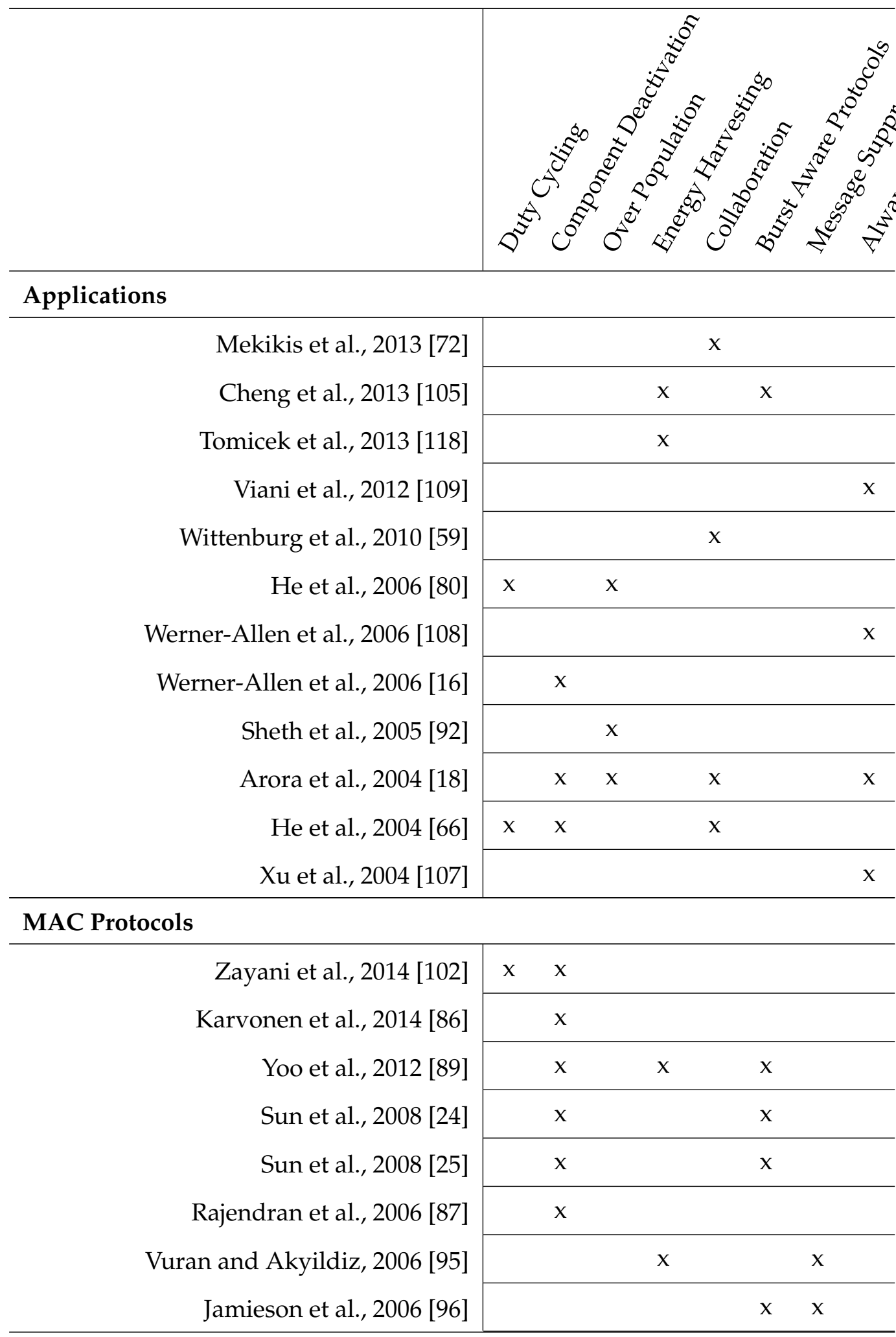

Continued on next page... 
Table 2.5 - Continued from previous page

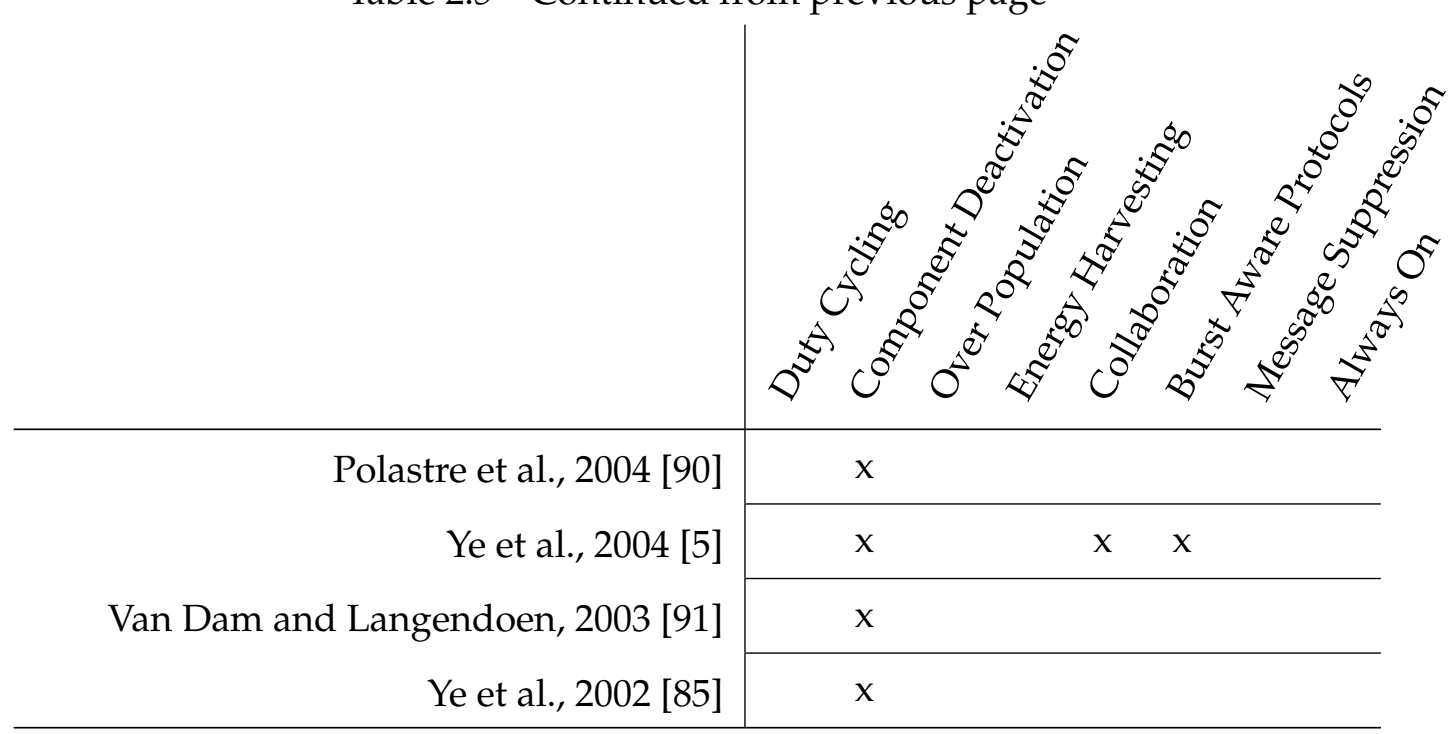

\section{Supporting Technologies}

Ammar and Reynolds, 2015 [114]

Tang et al., 2015 [120]

Misra et al., 2015 [12]

Das and Misra, 2015 [58]

Harrison et al., 2015 [1]

Rashid et al., 2014 [71]

Kavitha and Lalitha, 2014 [76]

Yang et al., 2013 [94]

Zhu et al., 2012 [75]

Milic, 2012 [74]

Alam et al., 2012 [73]

Alam et al., 2012 [77]

Kang et al., 2012 [79]

Thuc and Insoo, 2011 [68]

Wittenburg et al., 2010 [59]

Pripužić et al., 2008 [100]

Vigorito et al., 2007 [78]

Martincic and Schwiebert, 2006 [60]

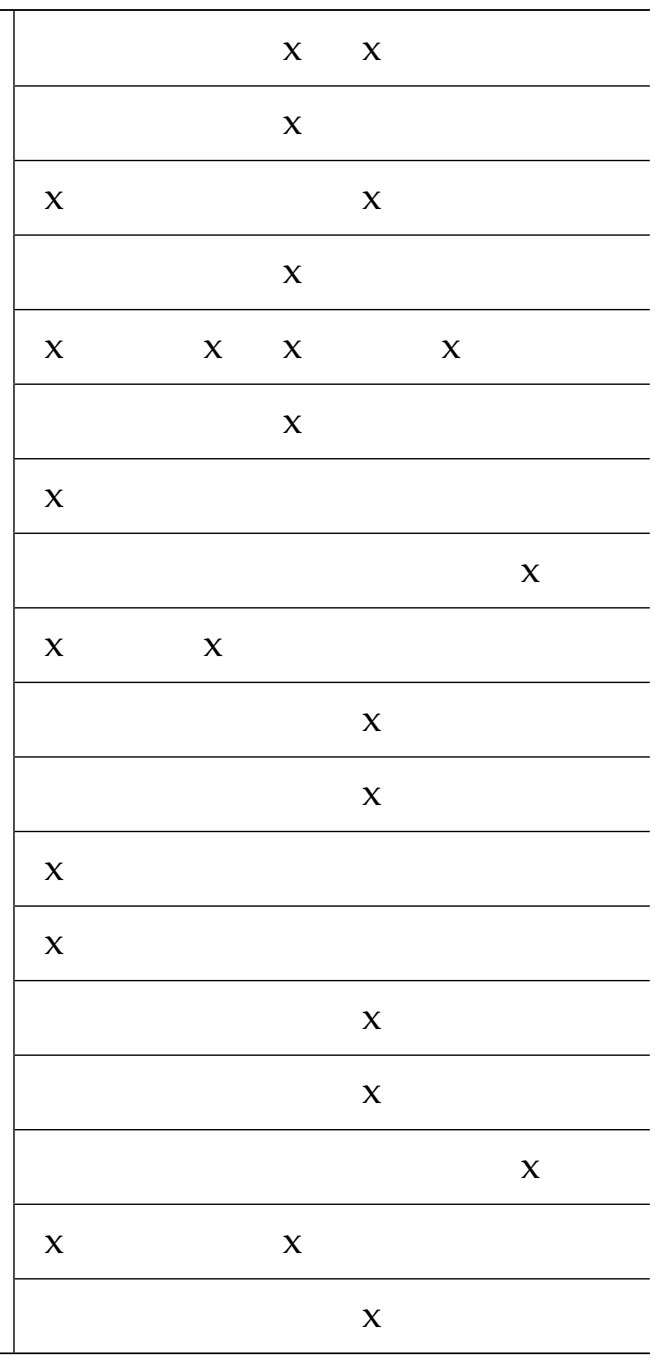

Continued on next page... 
Table 2.5 - Continued from previous page

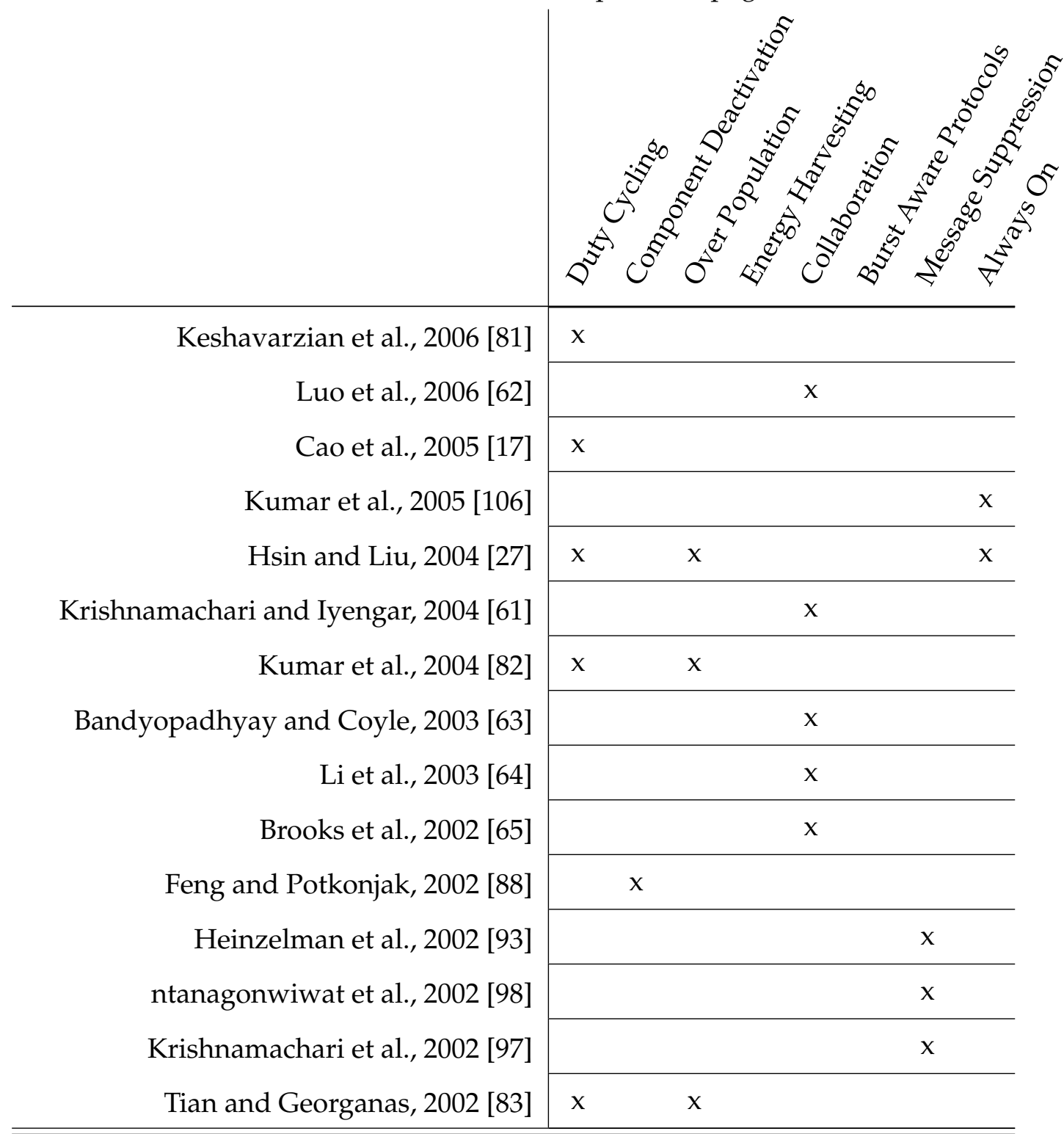

\subsubsection{Discussion}

WSNs can be classified by their data delivery profile as being continuous, eventdriven, observer-initiated (query-based) or a hybrid [23]. Based on the literature, it appears continuous, periodic sensing is the model that has been of most interest to WSN researchers, though event-sensing is increasing in popularity. The most 
widely cited ${ }^{1}$ survey on WSN research [121] briefly mentions event sensing and query based approaches, but the majority of the technologies and systems it surveys target periodic sensing deployments. A more recent survey [122] gives more space to event sensing and a survey of the state of the art of WSN programming techniques published within the last three years [123] features the application of event-triggered distributed processing.

Rare events are characterised by domain specific low occurrence probabilities, but selecting appropriate detection and propagation techniques relies on an understating of a number of other attributes:

Frequency While the probability of a rare event occurring in a given time period may be low, over an extended time period occurrences may become regarded as frequent. For example, the probability of an earthquake hitting New Zealand in the next given sixty seconds is low, yet the country experiences several hundred earthquakes a year.

Randomness To what extent do the events occur in a partially predictable fashion? In industrial settings there may be a trigger event such as certain piece of machinery being switched on that makes the rare event possible, but not necessarily probable.

Ephemerality How long a rare event lasts can inform strategies for dealing with it. If an event typically lasts for $n$ time units, is it acceptable to detect in the $n$th time unit? To what extent is it better to detect it in the 1st time unit?

Transitory Nature After an event occurs, is there any evidence it happened? If there is, is it acceptable to detect the effect rather than the event? A fracture in a water main can be sensed either as a physical change at the location of the break (potentially problematic for a long pipe) or as a drop in water pressure beyond the break.

Connectedness Once an event has occurred, does that imply something about the next occurrence? Can the magnitude or timing of subsequent events be predicted? Measuring the ephemerality of events as they occur could inform a sleep-scheduling algorithm that starts by assuming events are instantaneous but learns that the event always last (i.e. are detectable) for

\footnotetext{
${ }^{1}$ Citations as of October 7, 2015 - Google Scholar: 14,097, Microsoft Academic Search: 4,249.
} 
some period of time, hence the sleep cycle can be modified to be slightly less than that period.

Criticality How important is it that an event be detected? Once detected, how quickly must a notification message be propagated to interested parties? This may be related to event frequency, it may be acceptable to miss frequently occurring less critical events simply because it is a certainty another one will occur in the near future.

Network Lifetime How long must this network be active? Is the event so rare that once detected the network is permitted to effectively destroy itself by using all its energy to propagate the notification message.

To detect a rare event at a given location, at least one node within sensing range of that location must be operational when the event occurs. Similarly, once the event has been detected, sufficient nodes must be active such that a low latency route exists for forwarding event notification messages to the network sink. Whether powered by batteries or energy harvested from the environment, WSNs deployed to detect and report rare events are constrained by the twin requirements of maximising detection probability and minimising detection delay whilst working with a limited energy source. The event sensing strategies surveyed in this paper address these constraints, either individually or in complimentary combinations.

Two themes are highlighted in Table 2.5. Firstly, applications deployed for extended use, i.e. ones that do not adopt an always on strategy (Section 2.1.7), rarely rely on just one of the strategies presented here. Secondly, almost all MAC protocols for rare event sensing make use of component deactivation to the extend that they regularly turn off the wireless transceiver.

The predominance of multi-strategy real-world deployments is reflected in the number of citations of the surveyed papers. A the time of writing, the two most widely cited articles ${ }^{1}$ included in this section $[93,85]$ focus on single strategies: message suppression (Section 2.1.5) and component deactivation (Section 2.1.3) respectively. If the individual strategies are considered the building blocks of an efficient and successful WSN, which ones to use and how to combine them are fundamental considerations when developing real-world systems.

\footnotetext{
${ }^{1}$ Citations as of October 7, 2015 - Google Scholar: [93] 8,864 [85] 5,544. Microsoft Academic Search: [93] 1,991 [85] 2,222.
} 
In situations where event occurrence cannot be detected at an individual node or by a collaborative evaluation by neighbouring nodes, sensed data can be transmitted to a base station for consolidated event determination. A simulated mine safety system [124] takes this approach to detect gas leaks, water seepage and areas of high oxygen concentration that could provide refuge for mine workers in an emergency. Whilst the system as a whole detects rare events, the WSN nodes themselves perform periodic data capture only.

In addition to sensing geospatial phenomenon, malicious disruption of the WSN itself can be considered a rare event. [125] propose a decentralised three phase rules based algorithm for intrusion detection focusing on multiple attacks strategies including message delay, alteration and repetition. [126] and [127] describe the application of intrusion detection mechanisms to WSNs whilst [128] consider attacks on sensor-actuators in hostile environment to be rare events. Similarly, fault detection and tolerance in WSNs deployed to detect critical rare events $[129,130,131]$ are of significant importance.

Beyond dedicated WSNs, rare geospatial events can be monitored by collaborative use of personal electronic devices. Further, some classes of rare events can be predicted by off-line analysis of data captured from multiple heterogeneous sensor networks not originally deployed in a detection role.

Community Sensing [132] is an area of research investigating the use of the built-in sensors and communication capabilities of smart phones to detect and monitor rare events. The iShake App [133] and others [134] aim to assist in the detection of earthquakes and similar rare events with mobile sensing devices. Feeds from social networking sites have also been evaluated for their real-time "social sensing" potential [135]. 


\subsection{Coverage and Connectivity Algorithms}

All WSNs deployed for rare event detection have two related responsibilities: They must maintain sensing coverage so events are captured and ensure network connectivity so sensed data can be transmitted to base stations or fusion centers. Comprehensive surveys have been made of WSN coverage and connectivity [136, $137,138,139]$ with significant attention given to energy efficiency.

However, a recent coverage \& connectivity survey [140] did not identify energy harvesting as an area of current or future research. Similarly, a recent survey [111] did not find any rare event sensing application taking advantage of energy harvesting. A study of schedule-based asynchronous duty cycling in WSNs [141] did not consider event sensing and restricted itself to battery power. One paper that does address energy harvesting [78] adopts full node duty cycling and does not address rare event sensing specifically, yet does consider the implications of duty cycle variance over time as it relates to event sensing with the focus being on energy neutrality and duty cycle invariance between nodes.

Optimal coverage analysis dates back to a proof of the minimum number of circles required to cover a set [142] with more recent work specifically considering WSN deployment of sensors assumed to have circular sensing areas [143, 144, $145,146]$. Irregular sensing areas have also been considered [147, 148] as has energy efficient area coverage for sensors with adjustable ranges [149] yet circular or disk coverage remains most prevalent in the literature.

Energy efficient schemes for maintaining sensing coverage have been proposed [150, 26, 151] alongside coverage aware self-scheduling duty-cycling algorithms [152]. However, algorithms that address coverage and connectivity, including deployment schemes tuned to optimise both [153], are of most interest as maintaining one without the other is unlikely to be of much practical use.

Proof of an important relationship between coverage and connectivity is given in [154], described in Section 2.2.2:

Under the condition that $R_{c} \geq 2 R_{s}$, a sensor network only needs to be configured to guarantee coverage in order to satisfy both coverage and connectivity.

where $R_{c}$ is the connectivity (transmission) range and $R_{s}$ is the sensing range of all nodes in the network. Note this relationship does not hold for unequal transmission or sensing ranges. Table 2.6 shows transmission and sensing ranges of components found in commercial WSN devices. 
Table 2.6: Range of WSN Components

\begin{tabular}{lc}
\hline & Range (m) \\
\hline Texas Instruments CC2420 Transceiver ${ }^{1}$ & 100 \\
Analog Devices ADXL345 3-Axis Accelerometer & 0 \\
Panasonic EKMB123112 Passive Infrared Sensor $^{2}$ & 10 \\
\hline${ }^{1}$ From pharos.ece.utexas.edu/wiki & \\
${ }^{2}$ From pewa.panasonic.com &
\end{tabular}

The remainder of this section will examine schemes for preservation of coverage and connectivity. Section 2.2.9 summarises the algorithms evaluated.

\subsubsection{Span: Energy-efficient Topology Maintenance}

Span [155] is not aimed at WSNs and, as such, does not consider coverage, yet it does provide a useful reference point for connectivity research. Spans aims to reduce energy consumption in multi-hop ad hoc wireless networks like WSNs without dimishing capacity or connectivity. The basic principle being that in shared channel wireless networks with an over population of nodes (Section 2.1.4) only a subset of those nodes need to be energized at any time to forward traffic for active connections. Span is a distributed algorithm, nodes making local decisions on whether to power down or join a forwarding backbone as a coordinator.

Span's randomized algorithm rotates coordination responsibilities over time whilst maintaining capacity and connectivity, as the ratio of sleeping to active nodes increases, system-wide energy consumption decreases and network lifetime is improved. Importantly, Span is not simply concerned with connectivity, the authors note that a connected network does not necessarily preserve throughput. This insight influences Span's coordinator eligibility rule, as shown:

Coordinator eligibility rule: A non-coordinator node should become a coordinator if it discovers, using only information gathered from local broadcast messages, that two of its neighbors cannot reach each other either directly or via one or two coordinators.

Conversely, a coordinator should withdraw if every pair of its neighbours can reach each other directly or via one or more other coordinators. Coordinators periodically check if they need to continue as such. To rotate coordination responsibilities fairly, after a node has been a coordinator for a period of time based 
on it's remaining stored energy, it marks itself as tentative if it could withdraw. If after a grace period determined by the size of the network the coordinator can still withdraw, it does so. Tentative nodes continue to act as coordinators, but are dropped from neighbour nodes calculations of their own coordinator eligibility.

As the time a node retains its coordinator responsibilities is related to its energy reserves (the more energy it has the longer it will be willing to act as a coordinator) network lifetime is optimised as nodes with restricted energy reserves act as coordinators less frequently. Figure 2.1 shows network lifetime result from a simulation undertaken by the authors comparing Span with varying node densities to IEEE 802.11 with and without power saving mechanism (PSM). Figures 2.2a and $2.2 \mathrm{~b}$ compare how much time nodes spend in coordinator role based on initial stored energy.

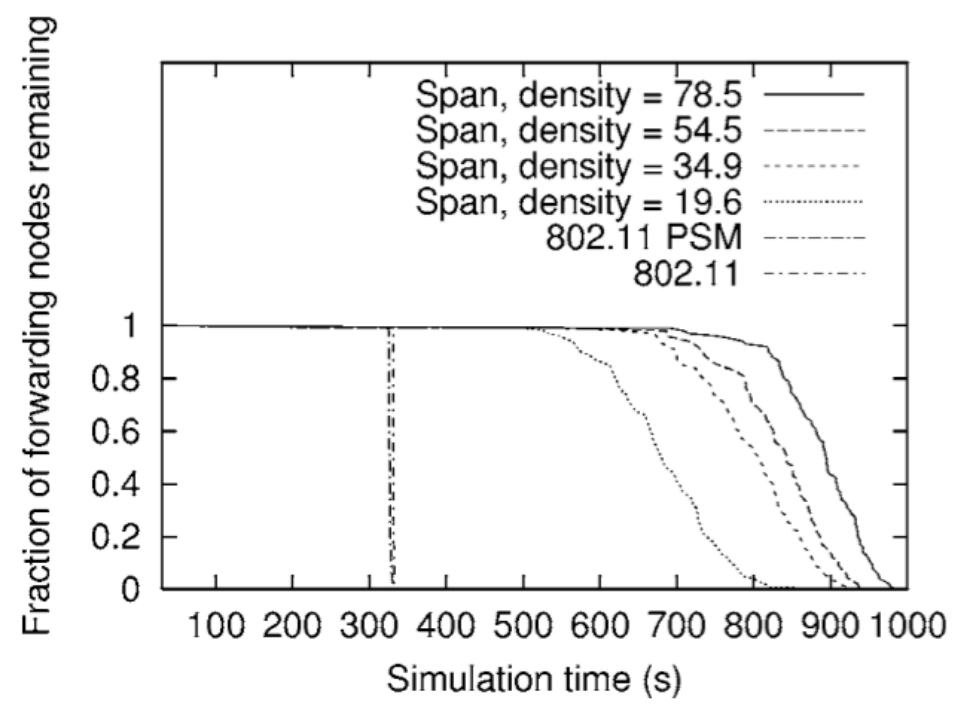

Figure 2.1: Span: Fraction of nodes remaining as a function of simulation time. With Span, nodes remain alive for significantly longer periods of time. [155] 


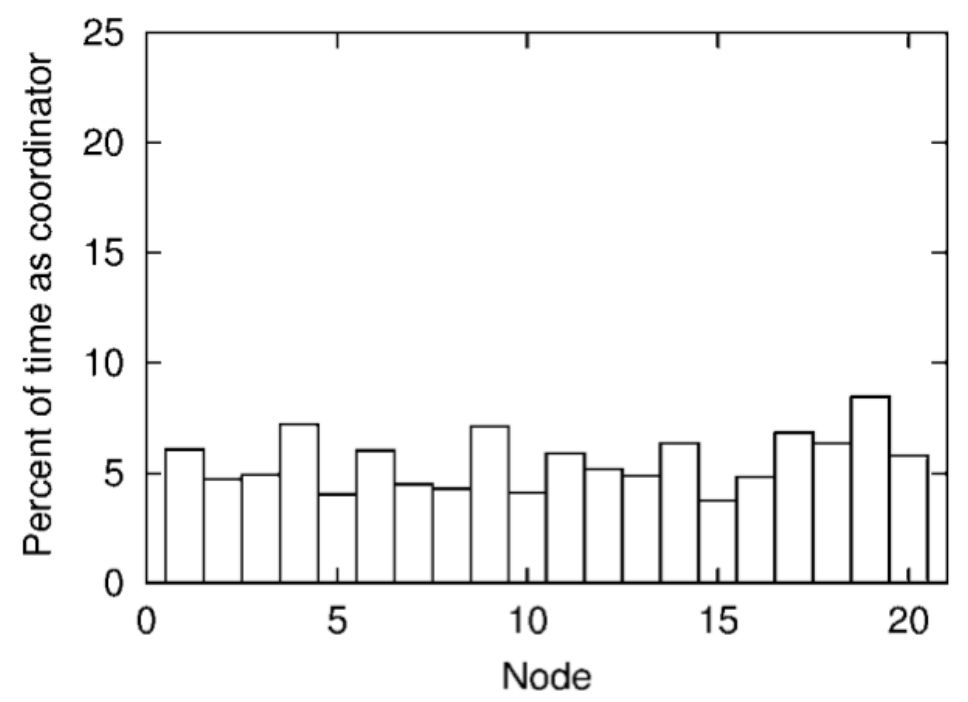

(a)

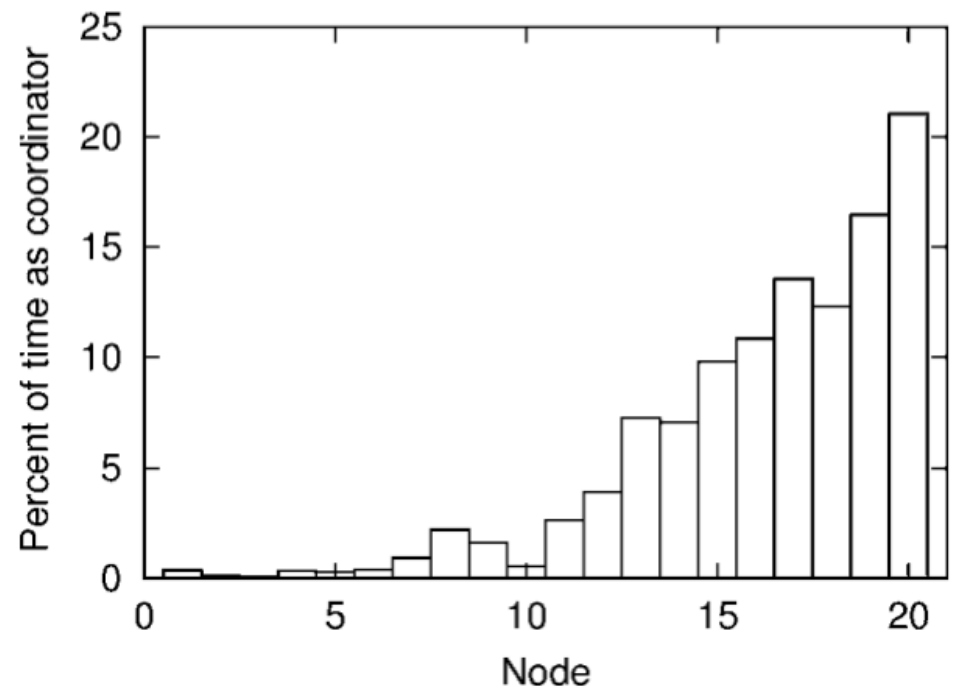

(b)

Figure 2.2: Span: Percentage of time each node in a 20 node, $100 \mathrm{~m}$ by $100 \mathrm{~m}$ network spent as a coordinator during 7200 s of simulation. In (a), each node starts with 10,000 J of energy showing Span rotates coordinators equally among all the nodes. In (b), each node starts with $2000+400 \mathrm{i}$ J of energy, where $\mathrm{i}$ is the node ID showing Span is more likely to elect coordinators with more energy. [155] 


\subsubsection{CCP: Integrated Coverage and Connectivity Configuration}

CCP [154] is a configuration protocol guaranteeing application defined degrees of sensing coverage and network connectivity, the work arguably being most significant for its geometric analysis of the relationship between connectivity and coverage in networks made up of nodes with uniform sensing and transmission ranges. For sensing range $R_{s}$ and communication range $R_{c}$ the authors show that:

- Theorem 1 For a set of sensors that at least 1-cover a convex region, the communication graph is connected if $R_{c} \geq 2 R_{s}$.

- Theorem 2 A set of nodes that $k$-cover a convex region forms a $k$-connected communication graph if $R_{c} \geq 2 R_{s}$

- Theorem 3 or a set of sensors that $k$-cover a convex region, the interior connectivity is $2 k$ if $R_{c} \geq 2 R_{s}$.

- Theoroem 4 A convex region $A$ is $k$-covered by a set of sensors if (1) there exist in $A$ intersection points between sensors or between sensors and $A$ 's boundary; (2) all intersection points between any sensors are at least $k$ covered; and (3) all intersections points between any sensor and $A$ 's boundary are at least $k$-covered

Theorem 4 is the $k$-coverage eligibility rule, an example of 1 -coverage eligibility being given in Figure 4.2a, the node with the highlighted sensing area is eligible to enter sleep mode as its sensing area is completely covered by neighboring nodes.

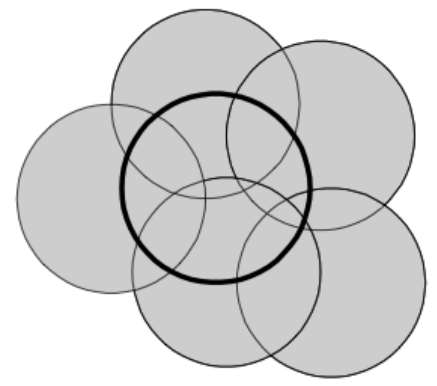

Figure 2.3: An example of 1-coverage eligibility [154]

CCP guarantees coverage and connectivity only if $R_{c} \geq 2 R_{s}$. When communication range is less than twice sensing range, incorporating Span (Section 2.2.1) allows connectivity to be maintained. The eligibility rules now become: 
- Eligibility rule for inactive nodes: An inactive node will be eligible to become active if it is eligible according to the eligibility rule of SPAN or CCP.

- Eligibility rule for active nodes: An active node will withdraw if it satisfies the eligibility rule of neither SPAN nor CCP.

The authors ran simulations of 20 source and sink nodes, all of which start with 5000 Joules of energy, each source node sending CBR traffic at 3Kbps. Deployment densities of 200, 250 and 300 are used for the remaining nodes in the experiments. With each density, the nodes are randomly distributed in a $400 \mathrm{~m}$ by $400 \mathrm{~m}$ network field and each of them starts with an initial energy selected randomly within the range from $200 \mathrm{~J}$ to $300 \mathrm{~J}$. The ratio of communication and sensing range is fixed at 2.5. Figure 2.4 and Figure 2.5 show system coverage and communication lifetime of SPAN+CCP and original network with all nodes on, respectively.

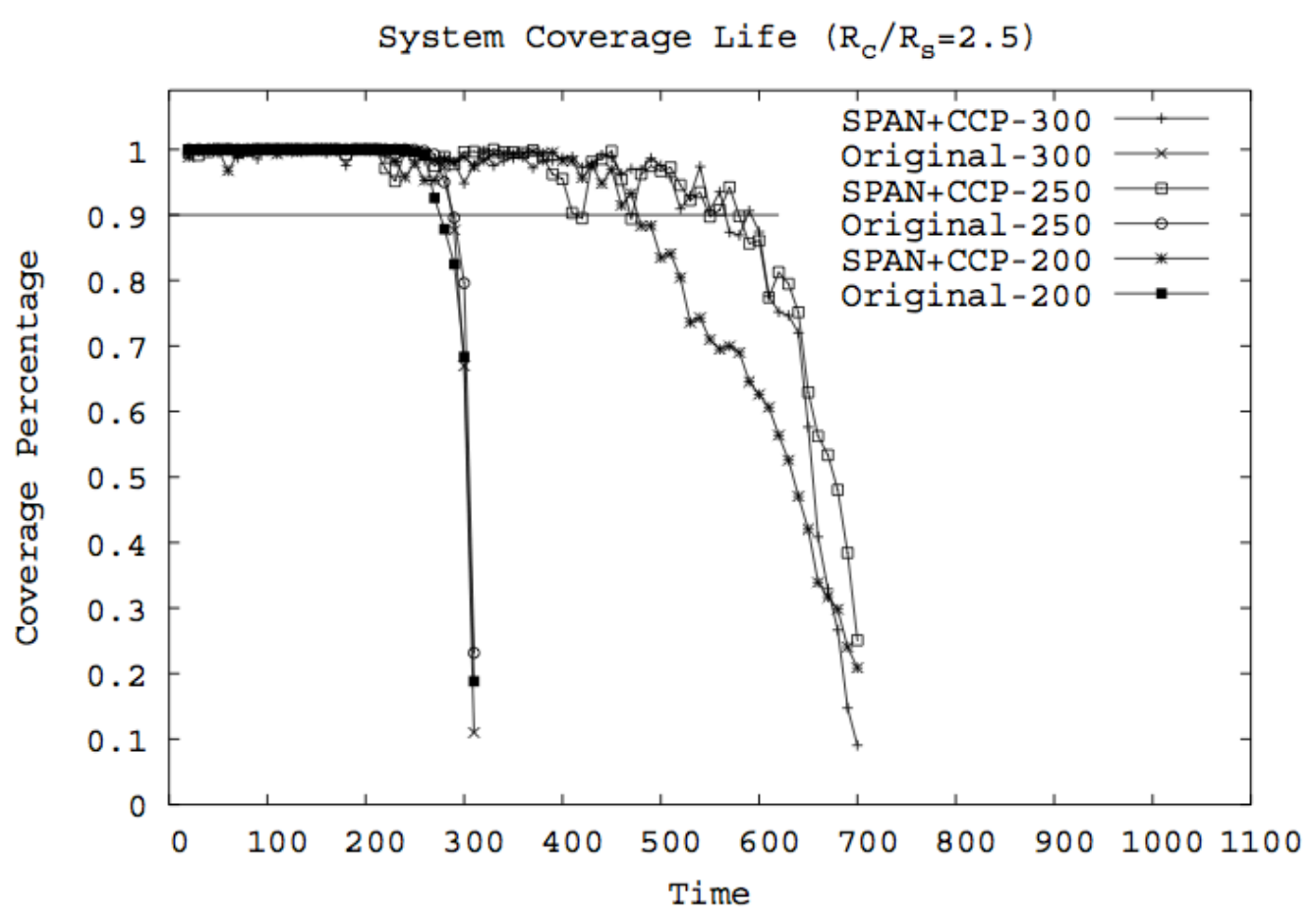

Figure 2.4: CCP: Coverage [154]

Whilst $100 \%$ connectivity is maintained for around $80 \%$ longer with CCP+SPAN compared to the original, always on (Section 2.1.7) network, initial coverage is not preserved beyond the point at which the original network looses coverage due to node death. Whilst some level of coverage is preserved for as much as twice as 


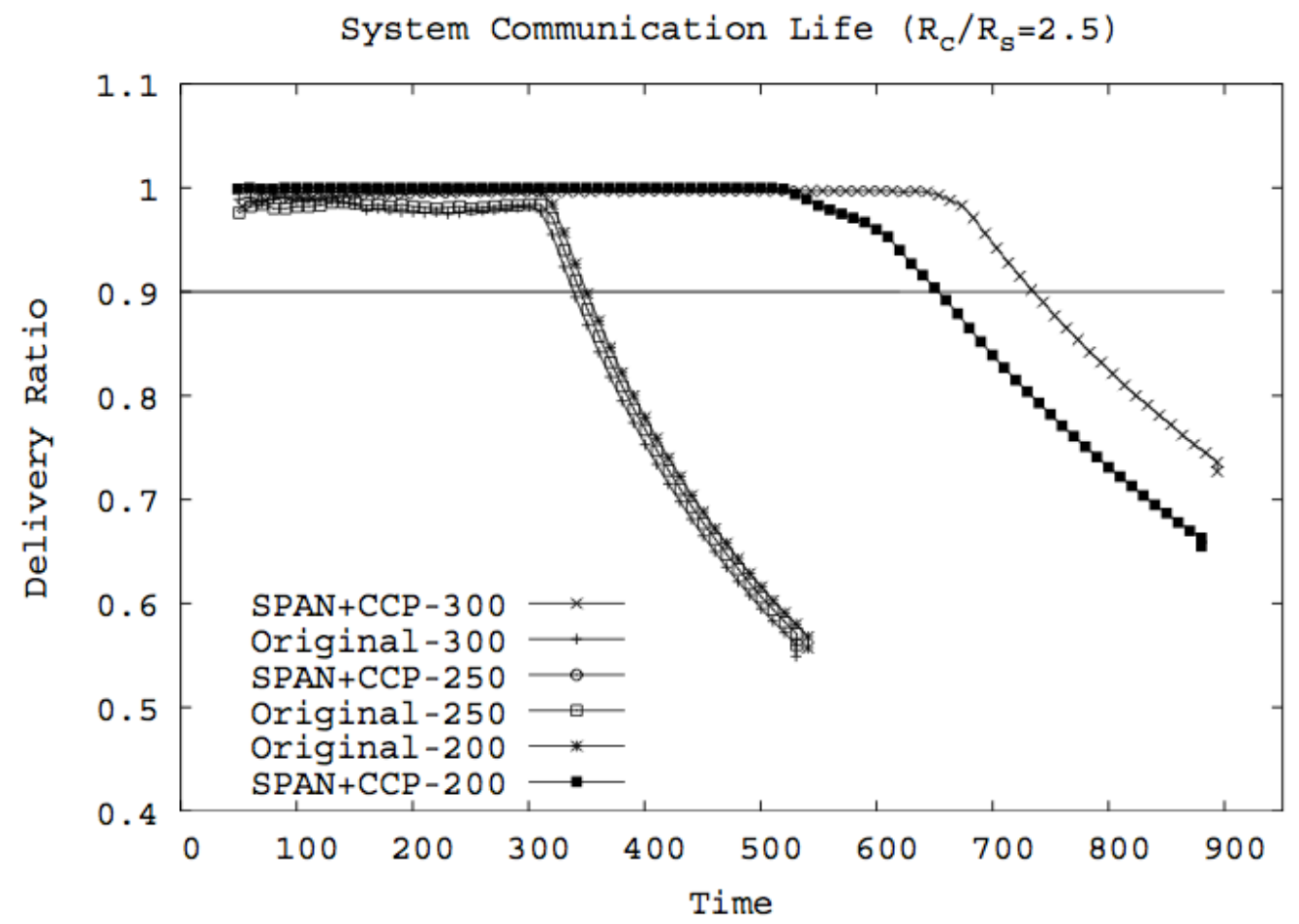

Figure 2.5: CCP: Connectivity [154]

long as the original network, CCP would appear a poor choice if retention of initial coverage were required.

\subsubsection{RACP: Role Alternating Coverage Preservation}

RACP [27] is a role alternating protocol for coverage preservation in networks where sensing and communication ranges are identical: $R_{c}=R_{s}$. Whilst RACP differs from CCP (Section 2.2.2) in its reliance on an simplified and arguably unrealistic relationship between sensing and communication ranges, the two protocols adopt similar theories on geometric determination of sleep eligibility, though the RACP authors, unlike their CCP colleagues, do not disclose details on the algorithm adopted.

Simulations are run to compare RACP to random sleep schedules and the coverage algorithm proposed in [83] (denoted CA). Figure 2.6 shows how the two algorithms compare for coverage in a square field of a given size $(L \times L \mathrm{~m})$ and known deployment density $(\lambda)$ for two values of sensing/communication range. Whilst RACP fares slightly better than CA with a sensing/communication range $(r)$ of 10 , when $r$ is set to 7, RACP provides little benefit as both algorithms suffer from simultaneous death of nodes located close to the sensing area boundary. 


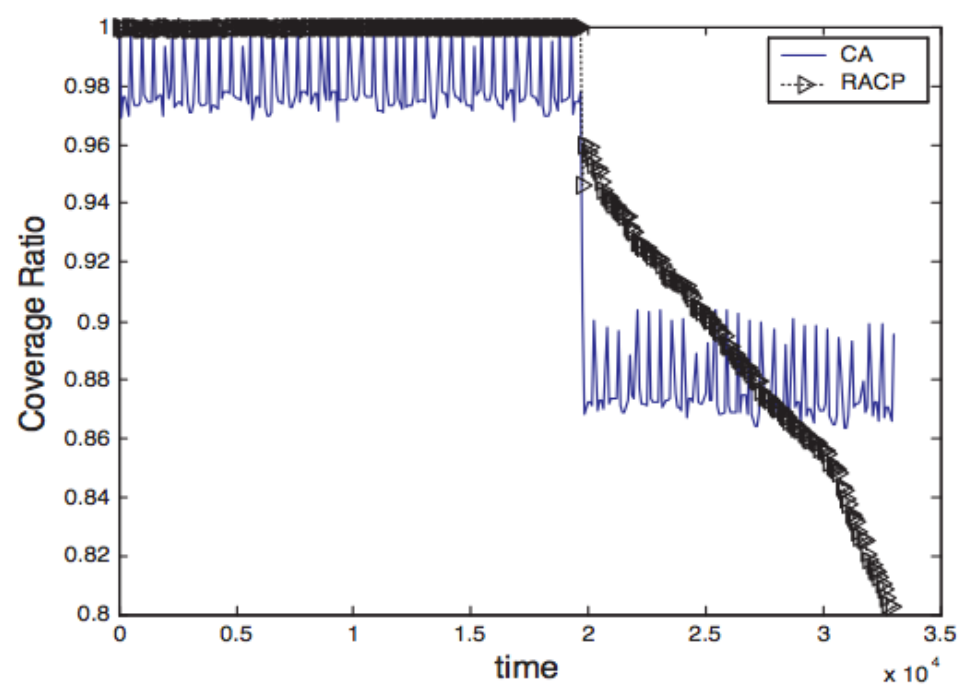

(a) $r=7$

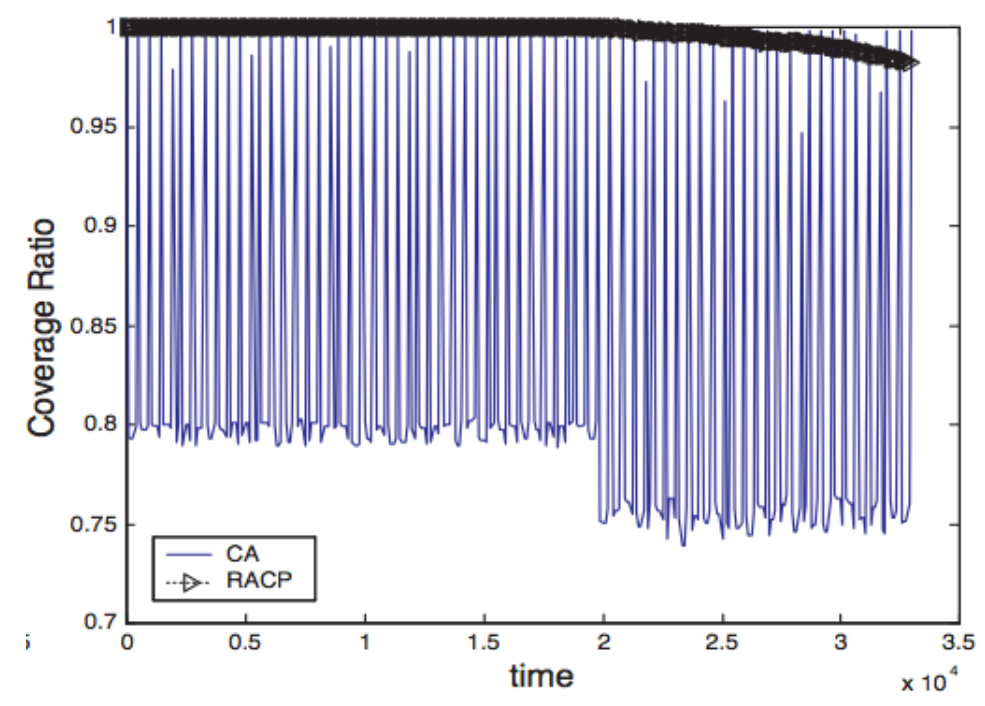

(b) $r=10$

Figure 2.6: RACP: Coverage ratio when $L=50, \lambda=0.06$ [27]

\subsubsection{OGDC: Optimal Geographical Density Control}

OGDC [156] maintains sensing coverage and network connectivity by keeping a minimum number of WSN nodes in active mode. In a similar manner to CCP (Section 2.2.2), the authors prove that when communication range is at least twice as large as sensing range, complete coverage of a convex sensing area implies connectivity amongst the set of active nodes. OGDC is fully localized and maintains coverage and connectivity regardless of the relationship between the radio range and the sensing range. 
Simulations show that OGDC outperforms contemporary algorithms [157, 154,158 ] with respect to the number of working nodes needed and network lifetime (with up to $50 \%$ improvement) but does not achieve the coverage demonstrated by the algorithm with the best result.

Simulations use the energy model from [158], where the power consumption ratio for transmitting, idle listening and sleeping is 20:4:0.01. One unit of energy is defined as that required for a node to remain idle for 1 second. Each node has a sensing range of 10 meters. Transmission range is at least twice the sensing range, set to $20 \mathrm{~m}$.

Coverage calculations are somewhat unique in that after dividing a $50 \times 50 \mathrm{~m}^{2}$ sensing area into 45 hexagons, as shown in Figure 2.7, the area is considered covered if the each hexagon is covered, hence $100 \%$ coverage is achevied if at least 45 sensors operate in each round, one for each cell. Beyond coverage, $\alpha$ lifetime, defined as the total time during which at least $\alpha$ portion of the total area is covered by at least one node, is measured and favourable comparison made to alternate algorithms as shown in Figure 2.9.

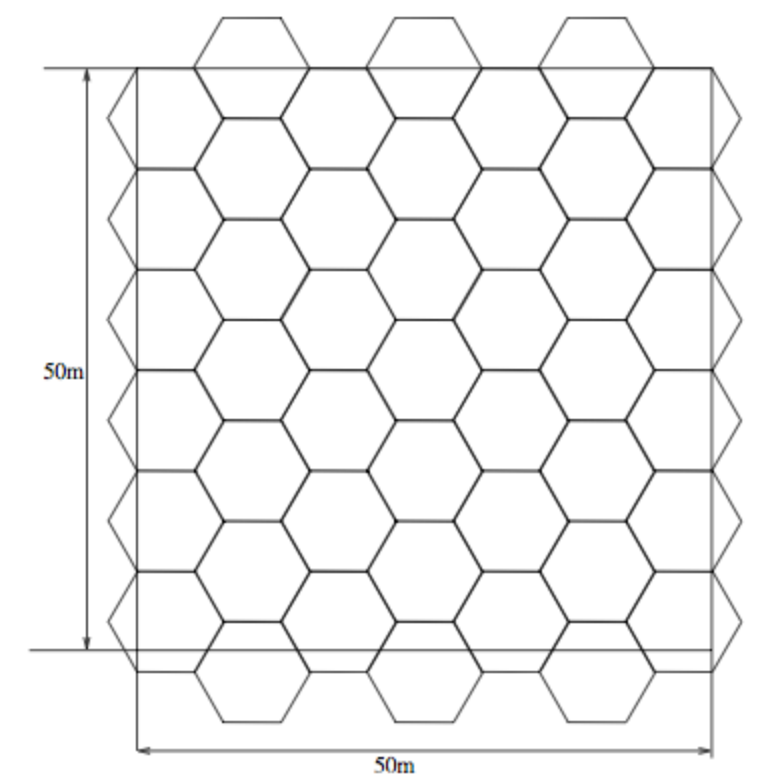

Figure 2.7: OGDC: 45 hexagons required to cover a $50 \mathrm{~m}^{2}$ square sensing area [156] 


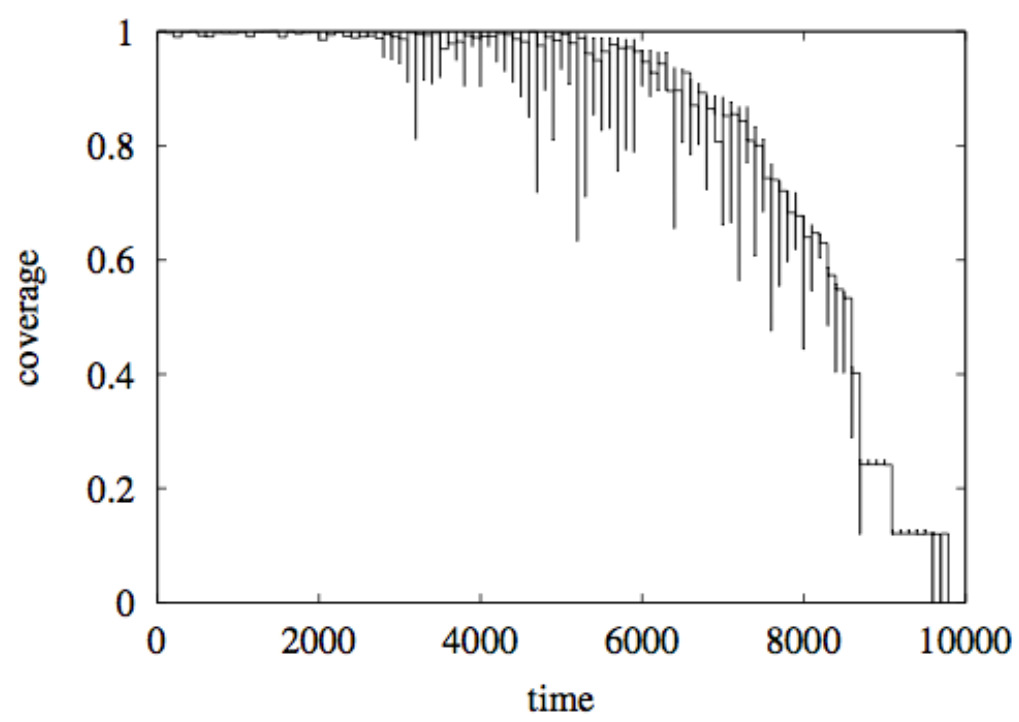

Figure 2.8: OGDC: Sensing coverage, 300 nodes in $50 \mathrm{~m}^{2}$ square sensing area [156]

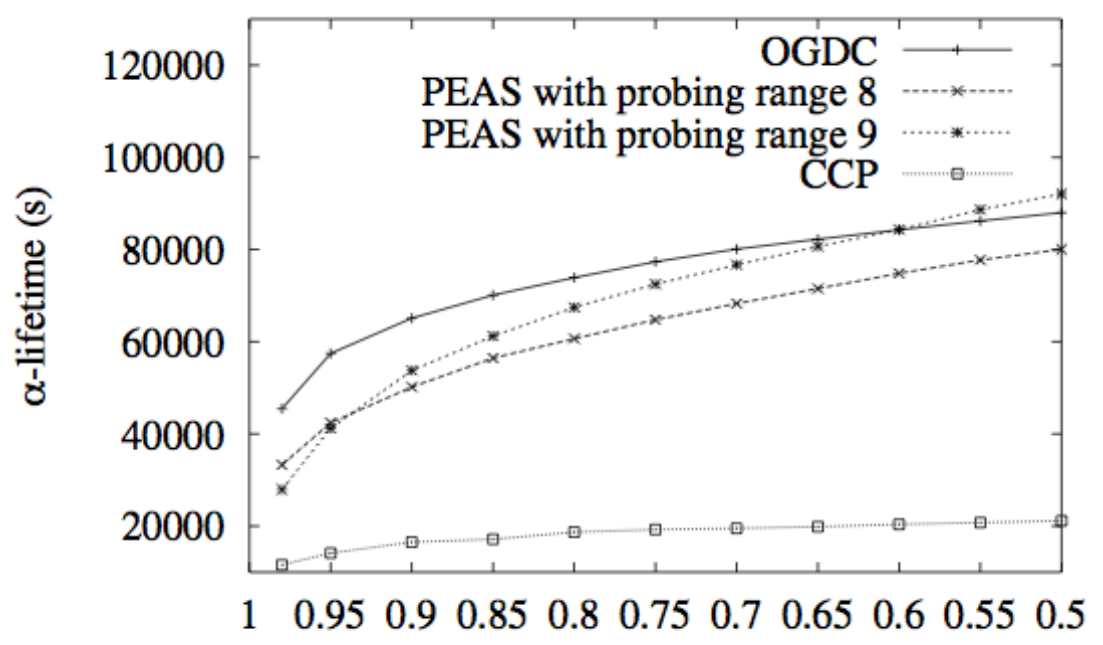

$\alpha$

Figure 2.9: OGDG: $\alpha$-lifetime against $\alpha$ [156]

\subsubsection{Sleep+PC: Distributed Coverage and Connectivity}

A duty cycling algorithm (Sleep) and a transceiver power controlling extension to it (Sleep+PC) [159] show a rare consideration of component deactivation (Section 2.1.3). Unlike like earlier works, less rigid assumptions are made about the relationship between sensing and communication ranges. Sleep is a distributed protocol for determining and adjusting $k$-coverage and $k$-connectivity in a WSN. Sleep+PC adds the concept of adjusting transceiver power instead of duty cycling 
the whole node.

The Sleep and Sleep+PC protocols are compared via simulation against a naive protocol where all sensors are always active, and the CCP+SPAN protocol [154] discussed in Section 2.2.2. Figure 2.10 \& Figure 2.11 indicate Sleep and Sleep $+\mathrm{PC}$ achieve similarly optimised $k$-coverage no more than twice the target where as the compared algorithms exhibit significantly sub-optimal $k$-coverage three or four times higher. Figure 2.11 indicates Sleep+PC performs better than the other algorithms with a $k$-connectivity effectively stabilised at the target value when Sleep on its own varies significantly over time averaging approximately four times the target value. CCP+SPAN is shown to be little better than the naive case with $k$-connectivity an order of magnitude higher than the target.

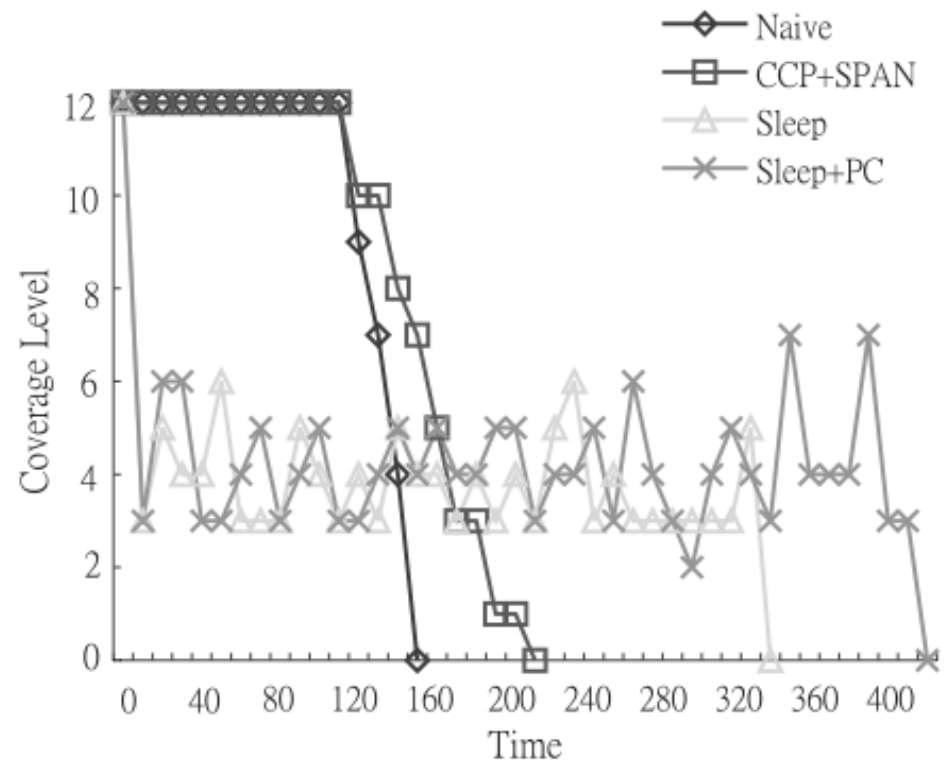

Figure 2.10: Sleep+PC: Coverage; target $k$-coverage $=3$, target $k$-connectivity $=2$ [159] 


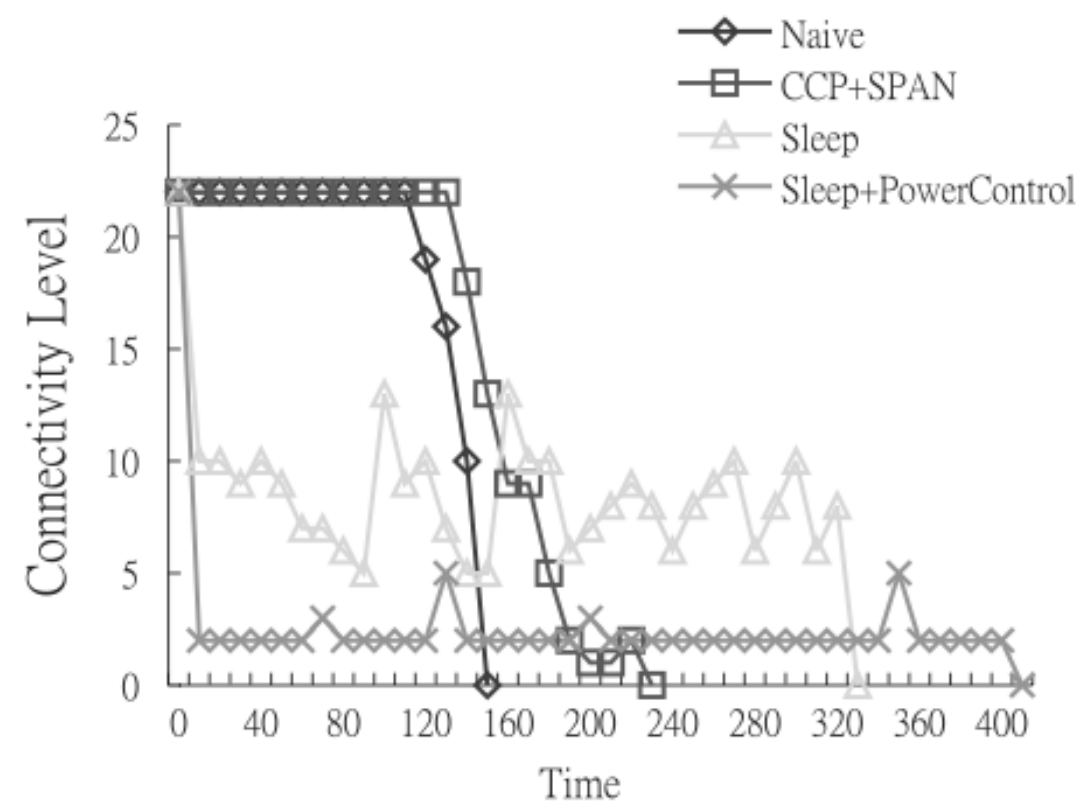

Figure 2.11: Sleep+PC: Connectivity; target $k$-coverage $=3$, target $k$-connectivity $=2$ [159]

\subsubsection{DLM: Distributed Lifetime Maximisation}

DLM [160] is a polynomial time distributed algorithm for maximising network lifetime to within no less than $O(\log n \times \log n B)$ of the maximum possible lifetime where $n$ is the number of nodes in the system and $B$ is an upper bound of the initial energy of each sensor. Unusually, DLM does not need to know node locations, instead it relies on an understanding of the distances between neighbour nodes and their sensing ranges. Time is assumed to be tightly synchronised and in every slot, the algorithm first assigns a weight to each node related to the fraction of its initial energy that has been used thus far. Then, in a distributed manner, it finds an $O(\log n)$ minimum weight sensor cover, which it activates in the slot.

Emphasis is given to the distinction between the arbitrary polygon that bounds the area to be monitored and the target field which includes the portion of node sensing areas that extend beyond the monitoring area. Deliberate placement of nodes outside both the monitoring area and the target field is required, introducing a third boundary, that of the node distribution area as illustrated in Figure 2.12. The target field is the area defined by the closure of the union of the sensing ranges of all the internal sensors and is assumed to subsumes the monitoring 
area.

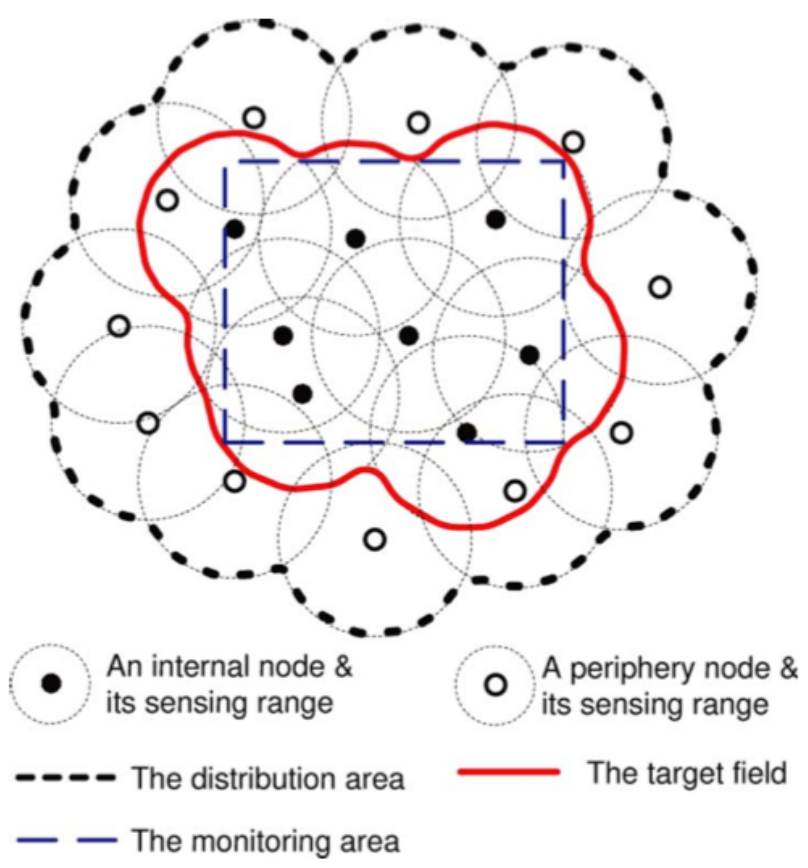

Figure 2.12: DLM: Target field in a small WSN [160]

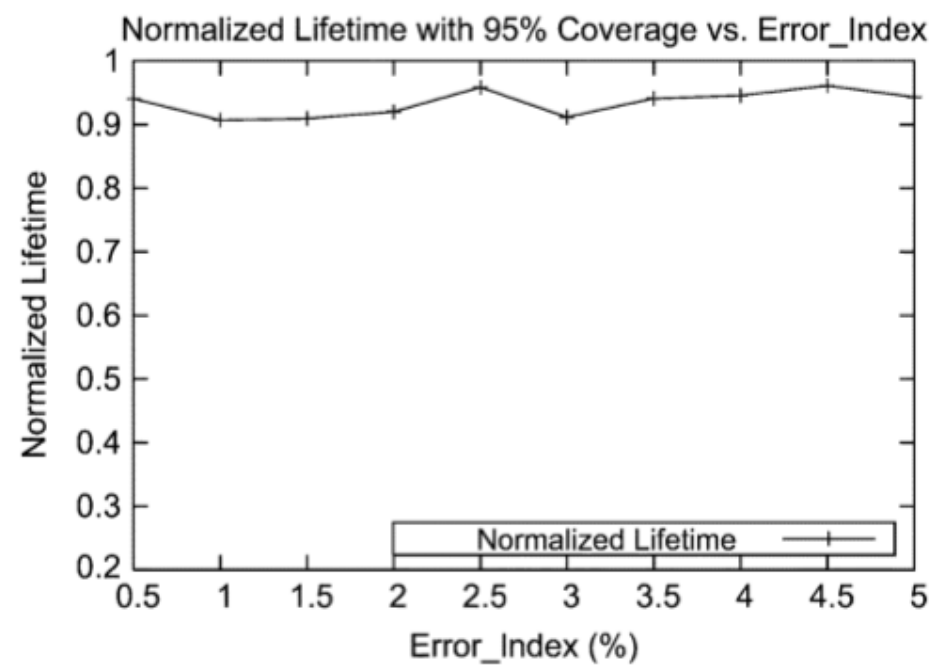

Figure 2.13: DLM: Normalized lifetime versus error index where $n=5$ and $B=$ $15[160]$

A challenge faced by DLM lies in it's assumption that nodes have an accurate record of the distances between pairs of adjacent nodes. In practice, this is unlikely to be the case and will prevent DLM from providing coverage guarantees, 
yet DLM's motivation is network longevity, not deterministic $k$-coverage. Figure 2.13 shows the effect of random errors in assessed inter-node distances.

\subsubsection{CAS: Optimized Event Detection in Low Duty-cycled Net- works}

CAS [75] is a fully localised cooperative scheme for scheduling event sensor node duty cycles without relying on location information. The related concepts of $d e-$ tection probability and detection delay are considered and CAS is shown, though simulation, to reduce detection delay by as much as $31 \%$ and increase detection probability by $25 \%$ when compared to a random independent scheme. The fact that no comparison is made to existing connected coverage algorithms is disappointing, but nevertheless, CAS has some interesting aspects, notably its nonreliance on location data and its focus on event sensing. Assumptions made by the algorithm are:

- Binary detection model. Each sensor has a sensing range. An event is reliably detected by an active sensor if its distance to the sensor is less than the sensing range.

- Time synchronisation. A mechanism for loose time synchronization should be available.

- Stationary events. After an event has occurred, it remains at the location where it happened. For events caused by moving objects, if the velocity of objects is slow, the approach may still apply.

- Inter-node distances are known. Every sensor is aware of the distance to each of its neighbors with no errors.

The CAS algorithm is:

Before the sensor network starts detecting events, CAS is executed to schedule sensor wakeups at the initialization stage. After CAS finishes, each sensor has determined its wakeup and enters the detecting stage, in which the sensor is alternatively in active mode and sleep mode. In each cycle, a sensor wakes up once at the wakeup time determined at the initialization stage and detects any potential event within its sensing vicinity. 
To prevent neighbour sensors waking up at the same time and duplicating each others effort, they must cooperate. CAS introduces a configuration parameter, the Cooperative Range (CR). Neighbours beyond CR, though within communication range are no considered a cooperative neighbour and do not influence a given node's sleep decisions - coordination message received from nodes further away than CR are ignored.

Simulations are run in a square field of side $300 \mathrm{~m}$, randomly populated with 3,600 sensing nodes (25 nodes per $\mathrm{m}^{2}$ ) each having a sensing range $\left(R_{s}\right)$ of $8 \mathrm{~m}$ and a communication range $\left(R_{c}\right)$ of $20 \mathrm{~m}$. Figure 2.14 shows detection delay and Figure 2.15 shows detection probability over time.

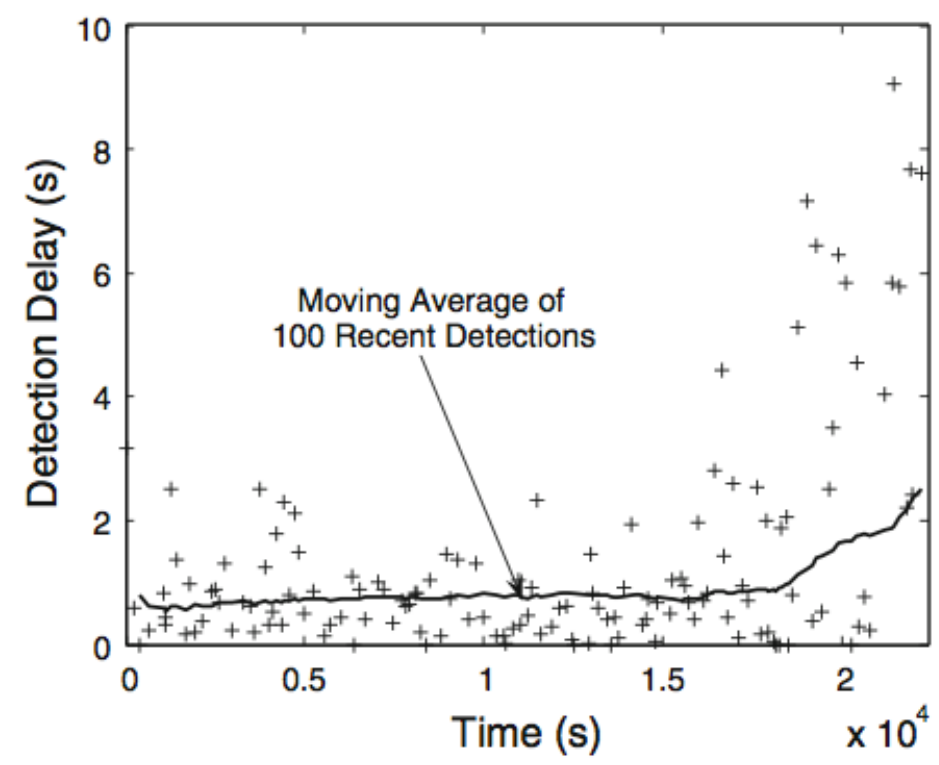

Figure 2.14: CAS: Detection delay, $10 \%$ cycle, $R_{s}=8 \mathrm{~m}, R_{c}=20 \mathrm{~m}, C R=1.2 R_{s}$ [75]

Whilst CAS exhibits a number of novel attributes, most noticeably the lack of reliance on accurate location data, its inability to maintain sensing coverage (as indicated by the lower than 100\% detection percentage in Figure 2.15) makes it an unsuitable starting point for an algorithm positioning coverage as a prime quality metric. 


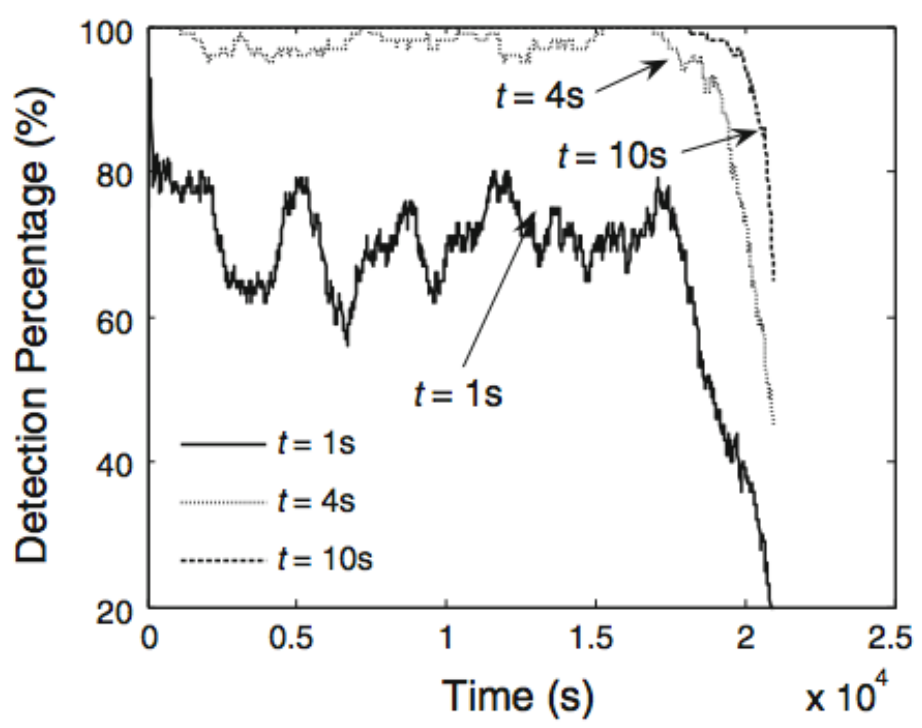

Figure 2.15: CAS: Detection probability, $10 \%$ cycle, $R_{s}=8 \mathrm{~m}, R_{c}=20 \mathrm{~m}, C R=1.2 R_{s}$ [75]

\subsubsection{Other Algorithms}

Maintenance of a less stringent Quality of Service (QoS) metric than required for rare event sensing (preserving a minimum number of targets that can be covered by the network over a 24-hour period) has been achieved by adjusting sensing ranges to reduce energy consumption during periods of negligible or no energy harvesting [161]. This approach, whilst ensuring the network exhibits acceptable but degraded coverage, does not address the peculiar requirements of time critical rare event sensing where initial coverage and instantaneous connectivity must be maintained at all times.

Energy harvesting is a fundamental input to Linear Programing (LP) and Maximum Utility Algorithm (MUA) based solutions to determine sleep scheduling and maintain sensing coverage introduced as solutions to the Maximum Lifetime with Energy Harvesting node (MLCEH) problem [162]. The MUA solution realises network lifetimes $25 \%$ shorter than LP, but is less computationally intensive.

Wireless rechargeable sensor networks (WRSNs) distribute power from energy rich sources to energy poor sensing nodes, with distribution by Radio Frequency (RF) signals [163] being viable only in ultra low-power applications. Recent work [164] has shown planned deployment methods that take into consider- 
ation the physical characteristics of the recharging method can be more efficient than those that do not. Opportunistic Energy Trading (OET) where energy is transferred from an energy harvesting WSN to an adjacent, non-harvesting WSN has been proposed [165] and a simplistic form simulated, though it remains to be shown what effect OET would have on coverage generally and in rare event networks specifically.

Though not concerned with the rarity or criticality of events, a global power management approach for energy harvesting sensor nodes [166] proposes joint duty-cycle optimisation and transmission power control to work within the constraints of a limited and time-varying energy source. Simulations are performed using a battery state-of-charge model with distinct consumption settings for sensing, transmitting and forwarding operations. Significant advantages over previous schemes are demonstrated but sensing coverage is not evaluated.

A mechanism for extending the operational life of a sensing network through application specific ultra low-power overlays has recently been proposed [167]. An energy harvesting secondary network runs alongside the mostly sleeping primary. When an event is detected by low-power sensors in the secondary network, wake-up radio technology is used to start the primary network nodes which activate energy hungry sensors to confirm event occurrence. In the examined scenario, the primary network relays live video of intruders having been notified of movement by passive infrared sensors in the secondary. A potential issue for rare and critical event detection could be the increase in notification delay introduced by the warm-up-time of the primary network.

\subsubsection{Discussion}

The algorithms evaluated in the preceding sections, summarised in Table 2.7, provide valuable insight into the relationship between sensing coverage and network connectivity, propose a variety of approaches to the calculation of coverage, and present schemes that practical implementations can be based on. Those that consider event detection either do not specifically consider rare events or caveat their event definition to the extent that those under consideration could be considered less than rare. Little attention is given to component deactivation, most algorithms concentrating exclusively on full node duty-cycling. Most significantly, all evaluated algorithms assume battery power, none of them giving any consideration to energy harvesting. 
Table 2.7: Coverage \& Connectivity Algorithms

\begin{tabular}{|c|c|c|c|c|c|c|c|}
\hline & $\operatorname{Span}^{1}$ & $\mathrm{CCP}^{1}$ & $\mathbf{R A C P}^{1}$ & OGDC $^{1}$ & Sleep $+\mathrm{PC}^{1}$ & DLM $^{1}$ & CAS $^{1}$ \\
\hline Year & 2002 & 2003 & 2004 & 2005 & 2007 & 2011 & 2012 \\
\hline Citations $^{2}$ & 2,625 & 1,198 & 295 & 1,431 & 105 & 62 & 9 \\
\hline Connectivity & $\checkmark$ & $\checkmark$ & & $\checkmark$ & $\checkmark$ & $\boldsymbol{\sim}^{9}$ & \\
\hline Coverage & & $\boldsymbol{v}^{3}$ & $\checkmark$ & $\checkmark$ & $\boldsymbol{\nu}^{6}$ & $\boldsymbol{\checkmark}^{10}$ & $\boldsymbol{\sim}^{12}$ \\
\hline$R_{c} \geq 2 R_{s}$ & & $\checkmark$ & & $\checkmark$ & $\checkmark$ & $\checkmark$ & $\boldsymbol{\sim}^{13}$ \\
\hline$R_{c}<2 R_{s}$ & & $\checkmark$ & $\boldsymbol{\swarrow}^{4}$ & $\checkmark$ & $\checkmark$ & & \\
\hline \multicolumn{8}{|l|}{ Energy Harvesting } \\
\hline Rare Events & & & & & $\nu^{7}$ & & $\boldsymbol{\sim}^{14}$ \\
\hline Node Duty Cycling & $\checkmark$ & $\checkmark$ & $\checkmark$ & $\checkmark$ & $\checkmark$ & $\checkmark$ & \\
\hline Component Deactivation & & & & & $\boldsymbol{\sim}^{8}$ & & $\checkmark$ \\
\hline Random Placement & $\checkmark$ & $\checkmark$ & $\checkmark$ & & $\checkmark$ & $\checkmark$ & $\checkmark$ \\
\hline Deterministic Placement & & & & $\boldsymbol{\nu}$ & & & \\
\hline Location Ignorant & & & & & & $\boldsymbol{\sim}^{11}$ & $\boldsymbol{\sim}^{15}$ \\
\hline Non-uniform Sensing & & & & $\boldsymbol{\nu}^{5}$ & & & \\
\hline
\end{tabular}

${ }^{1}$ Sections 2.2.1 to 2.2.7

2 As indicated by Google Scholar, May 1, 2014

${ }^{3}$ Initial coverage not preserved beyond always-on node death

${ }^{4}$ Strictly, $R_{c}=R_{s}$

${ }^{5}$ Considered in text, but simulations assume uniform sensing ranges

${ }^{6}$ Does not preserve initial coverage

${ }^{7}$ Not necessarily rare

${ }^{8}$ Transceiver power reduction, not deactivation

${ }^{9}$ Energy model only considers sensing

${ }^{10}$ Does not guarantee k-coverage where $k \geq 1$

${ }^{11}$ Requires accurate clock synchronisation

${ }^{12}$ Acceptable detection probability requires unreasonably high node density

${ }^{13}$ Strictly, $R_{c}>2 R_{s}$

${ }^{14}$ Assumes non-transitory events

${ }^{15}$ Requires approximate clock synchronisation 


\subsection{Geographic Forwarding Algorithms}

Opportunistic forwarding algorithms for ad-hoc wireless networks have long been studied [168, 169, 170] yet the literature indicates no algorithm specifically targeting networks exhibiting perfect area coverage has previously been proposed.

Routing protocols in wireless networks can be classified as either proactive (constantly maintain route tables), reactive (determine routes only when needed) or a hybrid [171]. Proactive protocols typically maintain routing tables by sending and receiving topology messages, available routes between any two nodes being determined in advance of data transmission. Reactive protocols do not periodically share network topology but send out routing probes when data transmission to a particular destination is required, forwarding the data messages once the route has been determined.

An orthogonal category, position based routing [172], uses an understanding of the location of network elements to inform routing decisions which can be used to complement the others or work autonomously. For critical event sensing, routing protocols where the first post-event packet to reach the sink does not contain the sensed data can be regarded as sub-optimal, having too great an impact on detection delay.

In networks where network topology is constantly changing, either through node mobility [173] or duty cycling [174, 175], opportunistic, broadcast forwarding algorithms have been shown to provide better throughput than unicast routing protocols $[176,177]$.

Geographic (location-aware) forwarding has proved efficient when information on the network topology is unavailable, but nodes are aware of their own location and those of some or all of the other nodes in the network [178]. In the simplest case, nodes are aware only of their own location and those of the network sinks.

Existing opportunistic forwarding algorithms have a number of common themes: collision avoidance, energy conservation, and hole avoidance; examples of each are discussed in the following sections. Section 2.3.4 summarises the effect of each theme on detection probability and delay.

Whilst routing protocols typically use periodically broadcast control packets (beacons) to co-ordinate data packet transmission, beacon-less forwarding protocols are simpler and better suited for networks subject to topology changes. 
Beacon-less forwarding protocols vary in implementation detail but tend to follow one of the two patterns described in Algorithm 1 and Algorithm 2.

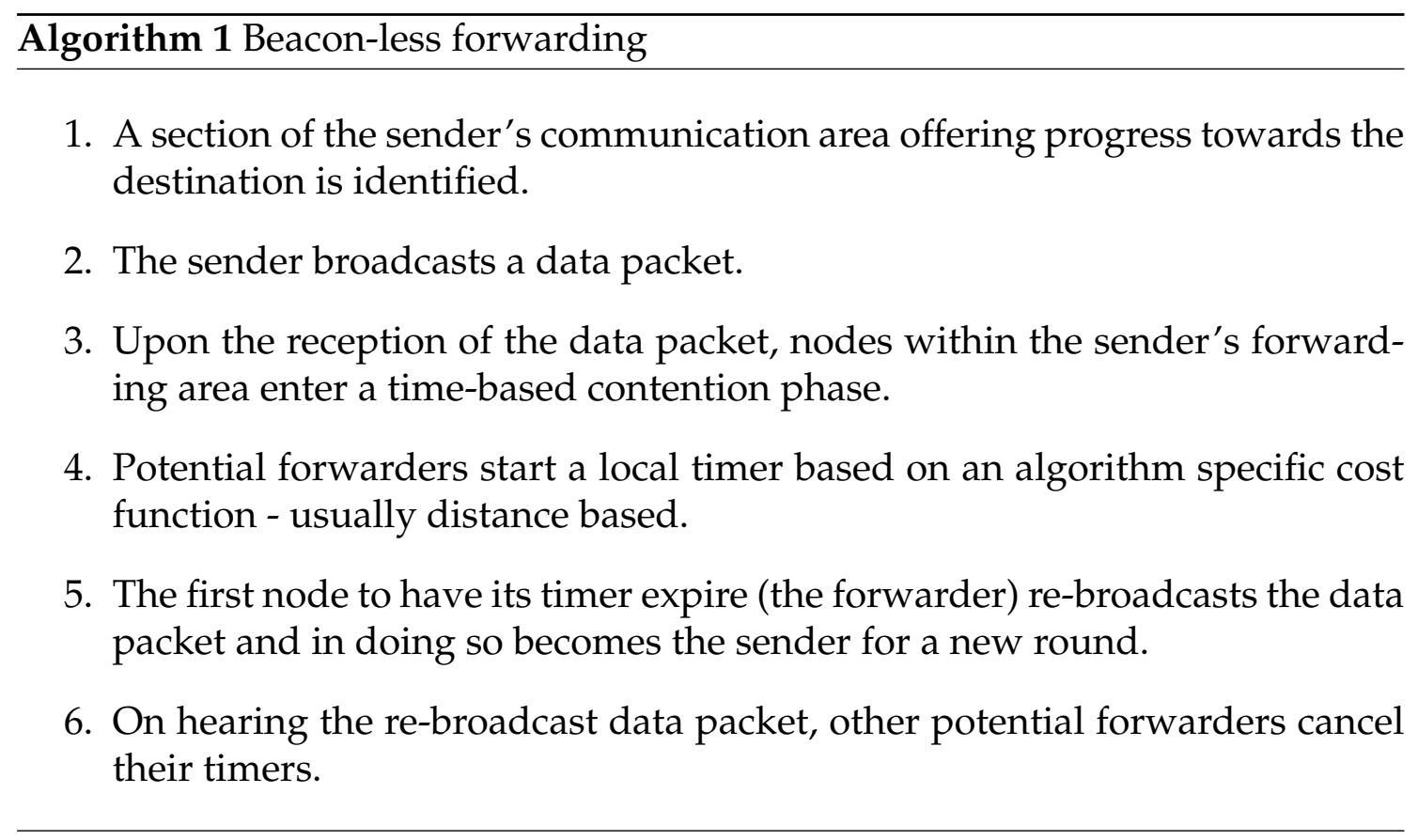

\subsubsection{Collision Avoidance}

Avoiding collisions is usually desirable as it reduces occurrences of dropped packets and avoids energy draining re-transmissions. An established ContentionBased Forwarding scheme (CBF) [179] is a classic implementation of Algorithm 1 that gives forwarding nodes in more advantageous locations priority, with nodes that are less well-positioned suppressing their forwarding transmissions. Suppression is achieved by introducing distance relative forwarding delays, the cumulative effect of which is to increase end-to-end transmission times.

The Beacon-Less Routing algorithm (BLR) [180] makes use of location information to reduce routing overhead without the need to periodically broadcast beacons. BLR selects forwarding nodes in a similar manner to CBF by employing a dynamic forwarding delay where only the most advantageously positioned nodes forward packets, all others suppressing (not forwarding) the packet when they overhear it being forwarded by a neighbour.

A more complex Beacon-less On-demand Strategy (BOSS) [181] is a variant of Algorithm 2 that uses multiple small messages to locally coordinate selection 


\section{Algorithm 2 Beacon-less forwarding with RTS/CTS}

1. A section of the sender's communication area offering progress towards the destination is identified.

2. The sender first broadcasts a small request-to-send (RTS) message.

3. Upon the reception of the RTS, nodes within the sender's forwarding area enter a time-based contention phase.

4. Potential forwarders start a local timer based on an algorithm specific cost function - usually distance based.

5. The first node to have its timer expire (the forwarder) transmits a clear-tosend (CTS) message.

6. On receipt of the CTS, other potential forwarders cancel their timers and the sender broadcasts a data packet.

7. When the forwarder receives the data packet it becomes the sender for a new round.

of the next forwarding node. However, the extreme caution taken to avoid collisions results in higher packet counts and increased latency that adversely impact detection delay.

Cost and Collision Minimizing Routing (CCMR) [182] is a combined MAC and forwarding scheme where the cost function of Algorithm 1 is based on locally calculated and generic cost metrics used to assign time slots to potential forwarders.

CoopGeo [183] includes a MAC-PHY cross-layer based on the centralized geographic approach in [184] where source-forwarder and forwarder-destination distances are used to select the most appropriate forwarders.

\subsubsection{Energy Conservation}

Minimising energy use is of significant importance to WSNs where power sources are finite and/or limited and network longevity is key. However, sensing critical rare events changes priorities to the extent that high but short duration forwarding costs become acceptable as they are incurred infrequently but deliver significant benefit.

The well established Geographic Random Forwarding (GeRaF) algorithm [185] 
and its multi-sink variant M-GeRaF [186], quite reasonably trade latency against energy usage, but for critical events minimal latency is key and energy consumption during the rare transmissions is of little significance compared to the energy involved in keeping the network connected by maintaining transceiver power.

An integrated MAC/ Routing protocol, MACRO [187] exploits the ability many WSN devices have to to transmit at discrete power levels and assumes energy savings when transmitting at lower levels. MACRO effectively implements Algorithm 1 but the cost function is based on a weighted progress factor, representing the progress towards the destination per unit of transmitted power. It should be noted that on the commercially available devices used throughout this thesis, MACRO's assumption that transmitting at lower power levels reduces consumed energy is entirely erroneous - as demonstrated experimentally in Chapter 3.

Implicit Geographic Forwarding (IGF) [188], though primarily aimed at mobile nodes, can be utilised in networks where topology changes due to collaborative sleep-scheduling rather than node mobility. IGF utilises a lazy binding technique where the locations of potential forwarding nodes are not known in advance of the sender wanting to broadcast a data packet. Essentially an implementation of Algorithm 2, IGF uses a distance based cost function in a forwarding region spanning a $30 \mathrm{deg}$ arc from the forwarding node towards the destination.

Opportunistic Routing in Wireless sensor networks (ORW) [189] specifically targets energy consumption as a key metric in duty-cycled networks. Packets are addressed to groups of potential receivers and forwarded by the one that successfully receives the packet when exiting sleep state. Propagation delay and energy consumption are reduced by significant margins when compared to alternate algorithms by utilizing all neighbors as potential forwarders, yet the remaining notification delay may prove too high for critical events.

A more recent opportunistic routing algorithm for asynchronous WSNs [190] assumes energy saving full-node duty cycling along the transmission path; transmission delays introduced by this otherwise sensible strategy may prove unacceptable for critical event sensing.

Opportunistic Flooding (OF) [191] is primarily intended for data dissemination across an entire network, but along with earlier flooding algorithms [192, 193] could be used for critical rare event forwarding as the additional cost of total network dissemination (as apposed to forwarding direct to a sink) is negligible given the rarity of the events in question. OF achieves reduced flooding delay and energy consumption over alternate algorithms, but focuses on low duty cy- 
cled networks not necessarily able to guarantee event detection.

\subsubsection{Hole Avoidance}

Algorithms for avoiding holes [194, 195, 196] attempt to route around geographical areas where the network is not connected. For rare event sensing, perfect event detection demands the sensing area be covered with active sensors, ensuring communication range is at least twice sensing range to achieve a connected network [154] with no holes.

\subsubsection{Discussion}

As when considering event sensing strategies in Section 2.1, forwarding priorities must be evaluated against their effect on detection probability and detection delay. Table 2.8 summarises typical effects of the forwarding priorities discussed in this section.

Many of the algorithms listed in this chapter use propagation speed as a comparison metric between themselves and others with similar priorities, but none prioritise delivery speed in and of itself. Whilst no previous work has focused on the unique situation introduced when sensing critical rare events, namely the need to minimize detection delay at all costs, collision avoidance algorithms that do not de-activate forwarding nodes promise the least impact on latency. 
Table 2.8: Typical Effects of Forwarding Priorities

\begin{tabular}{|c|c|c|c|}
\hline \multicolumn{4}{|c|}{ Detection } \\
\hline Priority & Probability & Delay & Observations \\
\hline $\begin{array}{l}\text { Collision } \\
\text { Avoidance }\end{array}$ & - & Increases & $\begin{array}{l}\text { Transport layer collision avoidance in- } \\
\text { evitably introduces forwarding delays } \\
\text { caused by nodes listening for transmis- } \\
\text { sions from neighbours better placed to } \\
\text { greedily forward packets. For realistic } \\
\text { node placements, forwarding delays in- } \\
\text { crease proportionally to the number of } \\
\text { hops required. }\end{array}$ \\
\hline $\begin{array}{c}\text { Energy } \\
\text { Conservation }\end{array}$ & $\begin{array}{c}\text { Can } \\
\text { Decrease }\end{array}$ & Increases & $\begin{array}{l}\text { Reducing energy consumption typically } \\
\text { involves turning nodes off for extended } \\
\text { periods. This not only has a detrimen- } \\
\text { tal effect on forwarding delay, but if im- } \\
\text { plemented by putting the entire node into } \\
\text { sleep mode is incompatible with the goal } \\
\text { of guaranteeing event detection. }\end{array}$ \\
\hline $\begin{array}{c}\text { Hole } \\
\text { Avoidance }\end{array}$ & - & $\begin{array}{c}\text { Can } \\
\text { Increase }\end{array}$ & $\begin{array}{l}\text { For perfectly covered networks the over- } \\
\text { head of hole detection is unnecessary. } \\
\text { Maintaining details on hole locations can } \\
\text { also be problematic in networks experienc- } \\
\text { ing frequent topology changes. }\end{array}$ \\
\hline
\end{tabular}

\subsection{Summary}

Whilst a significant amount of research has been undertaken on WSNs in general, the literature shows that issues other than event sensing have received the most attention. Further, within the body of work that focuses on event sensing, the criticality and rarity of the event is rarely considered.

The literature surveys summarised in these chapters make it clear that the combination of sensing rare events of critical importance with modest specification devices powered by small-scale energy harvesting has not previously been investigated. However, there is much to be learned from the surveyed literature; the algorithms presented in Chapter 4, Chapter 5, and Chapter 6 all draw inspiration from the work described in this chapter.

Specifically, Chapter 4 presents a collaborative duty cycling algorithm to main- 
tain coverage in an over populated sensing area. Chapter 5 introduces an opportunistic forwarding algorithm with embedded message suppression. The algorithm presented in Chapter 6 makes use of collaboration, duty cycling, over population, and message suppression. All three algorithms are targeted at always on, energy harvesting WSNs.

In Chapter 3, commercially available WSN devices are examined to determine their power consumption profiles in order to establish realistic simulation environments for the three proposed algorithms. 


\section{Chapter 3}

\section{Power Consumption}

Energy consumption is a preoccupation for Wireless Sensor Network (WSN) researchers whether the devices of interest are battery powered, harvest energy from the environment, or utilise some hybrid of the two. Put simply, the more energy the devices use, the less useful they become over time. When simulating WSNs to evaluate potential advances in sleep scheduling algorithms, low-power listening protocols, and opportunistic forwarding schemes to name just a few areas of active research, having a realistic model of how the devices under test consume energy is essential.

This chapter presents an assessment of the power consumed by the devices subsequent chapters use for simulated and live deployments of the algorithms they present. A power consumption model is generated from this evidence and an energy harvesting model driven by real solar radiation data is constructed. In addition, observations are made on how the results of the assessment have the potential to invalidate established areas of WSN research.

\subsection{Evaluation Setup}

A simple transmitter/receiver application was developed in NescC \& TinyOS [104] and cross compiled for the two commercially available low-power WSN devices that will be used in subsequent chapters, viz. the TelosB compatible Advanticsys MTM-CM5000-MSP (Advanticsys CM5000) [197] and the Unicomp UCMote Mini (UCMote Mini) [198]. Note that whilst only the Advanticsys CM5000 is simulated, both devices are used in the physical deployment described in Chapter 6. 
On initialisation or reset the transmitter waits a couple of seconds before energising its transceiver then waits a few more seconds before starting a $25 \mathrm{~ms}$ self-restarting timer to repeatedly broadcast an unencrypted 14 byte packet made up of standard TinyOS / IEEE 802.15.4 headers and a 2 byte payload. The receiver also waits a couple of seconds before energising its transceiver. On receipt of a packet broadcast by the transmitter, the receiver does nothing beyond assigning the value of the 2 byte payload to a program variable.

Both WSN devices and all measuring equipment were powered on for an hour before readings were taken to ensure everything was functioning at normal operating temperatures. The room temperature was $25.2^{\circ} \mathrm{C}$. For resistor shunt evaluations, the cable used was assessed to have a resistance of $1 \mathrm{~m} \Omega$ and the resistor plus cable was measured at $10.011 \Omega$ making the resistor $10.01 \Omega$.

\subsection{Measuring Current Draw}

To create an accurate energy model of a WSN device, it is fundamentally important to have measurements of how much current is drawn by the devices being modelled when the transceiver is switched off, when it is transmitting a packet, when it is receiving a packet, and when energised but neither transmitting nor receiving; this last state being know as idle listening [85].

Researchers have traditionally used Ohm's Law $(I=V / R)$ and a simple shunt resistor circuit to measure current draw, but on a number of occasions research colleagues have expressed an opinion that this technique is perhaps too simple to produce accurate results, even suggested the technique had been "discredited". To ascertain the accuracy of these claims, four additional measurement techniques where trialed to calculate the current draw when an Advanticsys CM5000 is transmitting and receiving a 14 byte packet:

Ammeter: Easiest method (Figure 3.1a); simply insert in the circuit and note the measured current. Ammeter was an Agilent U3402A digital multimeter.

Voltage drop across resistor shunt: The traditional method (Figure 3.1b); if voltage drop is measured with an oscilloscope, timed traces can be captured to see how long devices stay in a particular state. Ohm's Law allows direct calculation of the drawn current if the voltage and resistance are known. Voltmeter used was an Agilent DSOX2024A oscilloscope. 
Voltage before and after resistor shunt: Instead of measuring voltage drop across the resistor, the voltage before and after the resistor are measured (as shown in Figure 3.1c) using the Agilent U3402A digital multimeter and the Agilent DSOX2024A oscilloscope. The Ohm's Law calculation becomes $I=$ $\left(V_{1}-V_{2}\right) / R$.

Hall-effect sensor: The shunt resistor is replaced with a Honeywell CSLW6B40M, an open-loop current sensor with a sensitivity of $25.500 \mathrm{mV} / \mathrm{A}$ over a $\pm 40 \mathrm{~mA}$ range; see Figure 3.1d. The manufacturer's datasheet quotes resistance of the hall-effect sensor as $120 \Omega$ but when measured, the value was $87.067 \Omega$ giving a sensor plus wire resistance of $87.068 \Omega$. Hall-effect sensor evaluation was undertaken with automatic and manual calibration.

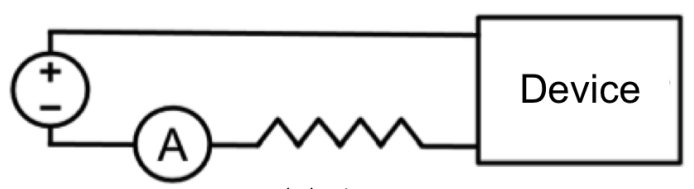

(a) Ammeter

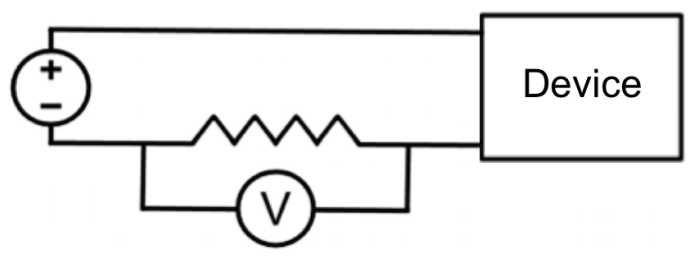

(b) Resistor shunt voltage drop

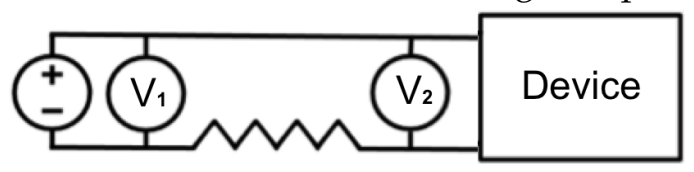

(c) Dual voltmeter/scope,

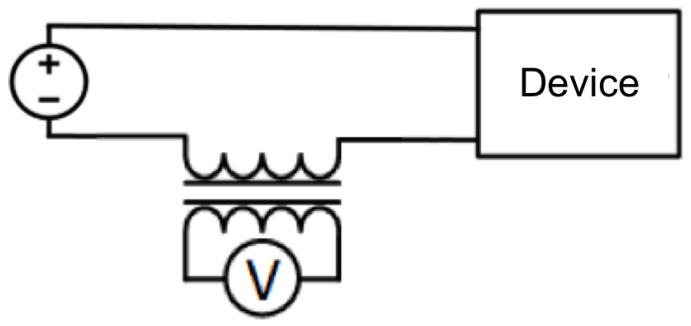

(d) Hall-effect sensor

Figure 3.1: Current draw measurement circuits 
Table 3.1: Current Draw by Measurement Technique, Advanticsys CM5000

\begin{tabular}{lcc} 
Technique & Transmit & Receive \\
\hline Manufacturer's Datasheet & $18.8 \mathrm{~mA}$ & $17.4 \mathrm{~mA}$ \\
Ammeter & $19.090 \mathrm{~mA}$ & $19.069 \mathrm{~mA}$ \\
Resistor Shunt Voltage Drop & $19.116 \mathrm{~mA}$ & $19.216 \mathrm{~mA}$ \\
Pre and Post Shunt Voltage & $19.031 \mathrm{~mA}$ & $19.306 \mathrm{~mA}$ \\
Hall-effect - Auto Calibration & $19.461 \mathrm{~mA}$ & $19.510 \mathrm{~mA}$ \\
Hall-effect - Manual Calibration & $18.986 \mathrm{~mA}$ & $18.934 \mathrm{~mA}$ \\
\hline
\end{tabular}

Results of these evaluations shown in Table 3.1 clearly indicate that there is little difference between measurement techniques, with no more than $2.5 \%$ variation in the results ${ }^{1}$.

\subsection{Transceiver Warm Up}

It can be tempting to assume energising the transceiver in a WSN device occurs instantaneously and for no energy cost; the voltage drop trace in Figure 3.2a would appear to support this theory. However, if the sample rate on the oscilloscope is increased by three orders of magnitude it becomes clear that it takes a finite time for the transceiver to become available, and during that time the energy consumed is approximately equivalent to the energy required to transmit a 14 byte packet. Point A in Figure 3.2b shows the timer kicking in, the transceiver not being ready for use until point $B$.

\subsection{Idle Listen vs Transmit \& Receive}

Manufacturer's datasheets can be misleading; the one for our Advanticsys CM5000 devices does not give a figure for idle listening, i.e. when the transceiver is energized, but neither transmitting nor receiving. A comprehensive paper on energy models for WSN devices [199] gives the "idle" power consumption of the CC2420 transceiver as $426 \mu \mathrm{A}$. While the accuracy of this value cannot be determined, our measurements show that when embedded in the Advanticsys CM5000, the entire device consumes approximately $19 \mathrm{~mA}$ when idle listening. From Figure 3.3a it is

\footnotetext{
${ }^{1}$ Measurements undertake with the assistance of Daniel Burmester and Tim Exley
} 


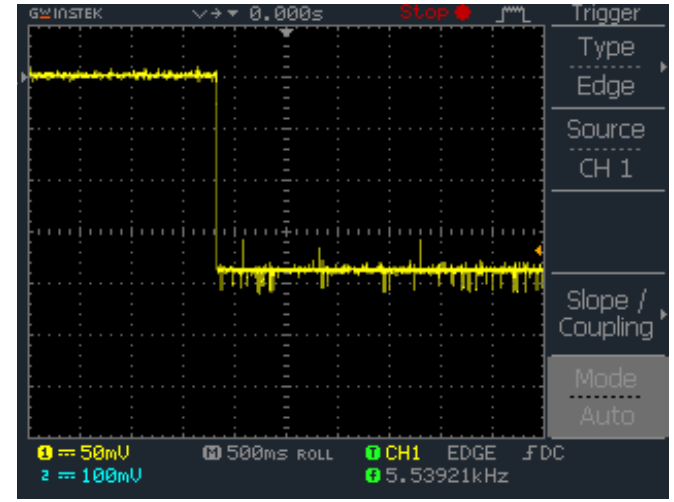

(a) 500 ms sampling

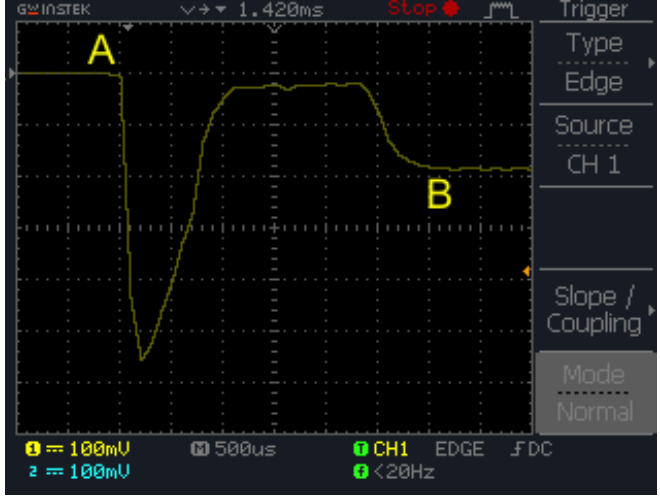

(b) $500 \mu$ s sampling.

Figure 3.2: Voltage drop across resistor shunt for timer based activation of the CC2420 transceiver in an MTM-CM5000-CSP.

clear that whilst receiving a packet increases the energy consumption of an Advanticsys CM5000 device by around 10\%, it only does so for approximately $1 \mathrm{~ms}$ and the device then returns to its idle listen current draw until the next packet is received. In the test system, packets are sent once every $25 \mathrm{~ms}$ meaning increased energy consumption of reception is experienced just $4 \%$ of the time. Hence, the total additional cost of receiving packets over simple listening for them, in this scenario, is only $0.4 \%$. Figure $3.3 \mathrm{~b}$ shows the additional cost of receiving packets on the UCMote Mini is even lower.

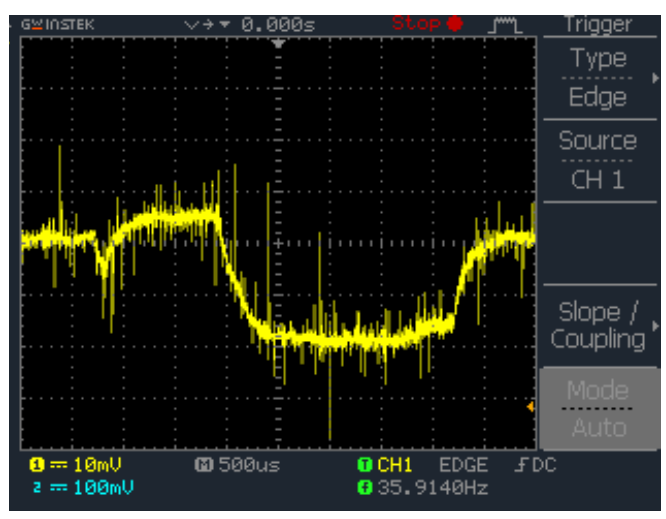

(a) MTM-CM5000-CSP

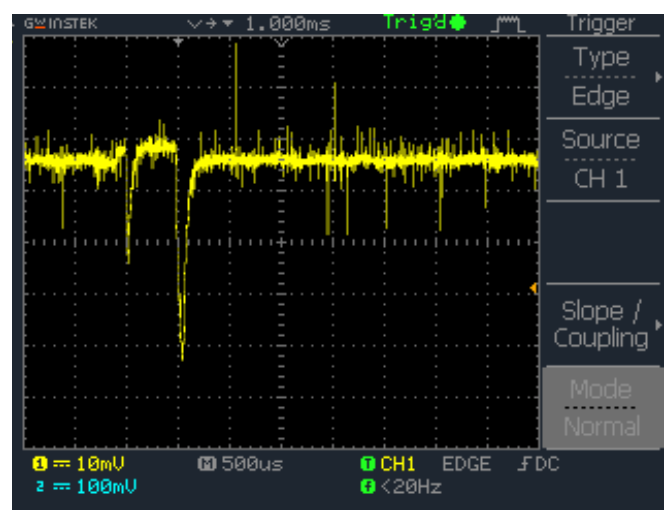

(b) UCmote Mini

Figure 3.3: Voltage drop across resistor shunt receiving a 14 byte packet. 


\subsection{Effect of Reducing Transmit Power}

Figure 3.4 shows the resister shunt voltage drop charts for the same Advanticsys CM5000 sending a 14 byte packet at maximum and minimum transmit power. Whilst the communication range is significantly different in each case, the energy consumed by the device is clearly unchanged.

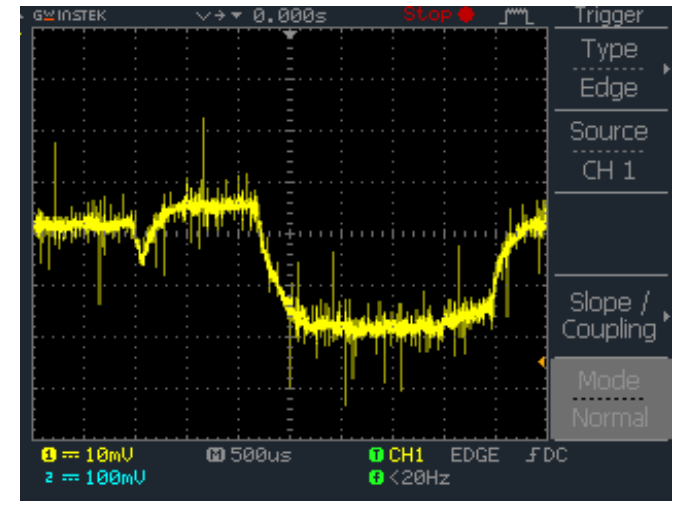

(a) Maximum transmit power

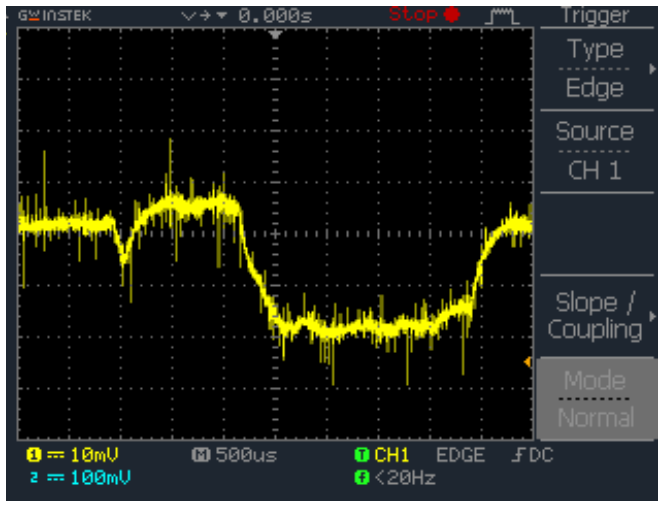

(b) Minimum transmit power

Figure 3.4: Voltage drop across resistor shunt sending 14 byte packet, MTMCM5000-CSP with CC2420 transceiver.

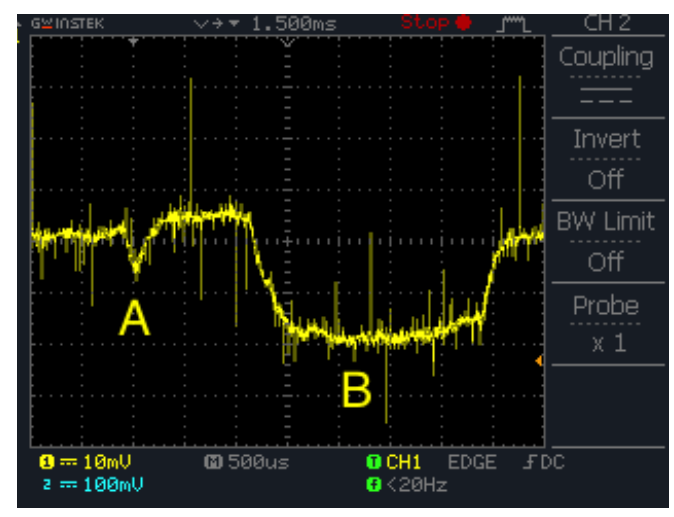

(a) MTM-CM5000-CSP / CC2420

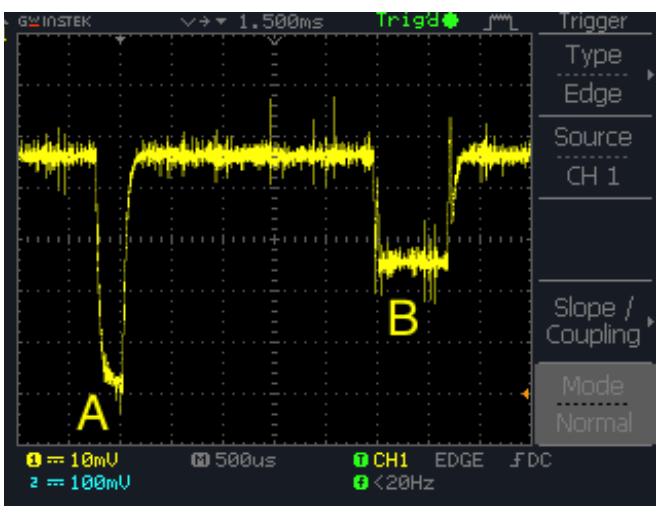

(b) UCMote Mini / ATMega128RFA1

Figure 3.5: Voltage drop across resistor shunt responding to a timer (point A) and sending 14 byte packet (point B) at default (maximum) transmit power.

\subsection{Device Differences}

Although small low-power WSN devices have similar aims and conform to internationally recognised communication standards, they vary significantly in their 
power consumption profiles. Manufacturer's choices of micro-controller, memory module, and radio transceiver create devices that vary significantly from one another in implementation, if not intent. Figure 3.5 clearly shows the differences between the devices we evaluated when sending a packet.

The UCMote Mini has an idle listen current draw of $13 \mathrm{~mA}$, which is $30 \%$ lower than the Advanticsys CM5000, Conversely, the UCMote Mini uses significantly more energy handling the timer interrupt (point A on Figs. 3.5b and 3.5a). When transmitting the packet (point B on Figs. 3.5b and 3.5a) the UCMote Mini draws as much additional energy over its idle listen consumption, but it does so for less than one third the time the Advanticsys CM5000 is consuming extra energy above its idle listen state.

\subsection{Power Consumption and Energy Harvesting Model for Finite State Simulations}

Energy available to sensing nodes is modelled as a linear charge store. As the nodes consume energy, charge is removed from the store; when harvesting energy during daylight hours, charge is added up to a maximum capacity. In practice, dual capacitor storage systems are required to facilitate simultaneous charge and discharge of the storage system [200].

When the charge held by a node is entirely depleted, it shuts down and the energy model waits until the store achieves $10 \%$ of initial charge before waking the node up to resume its sensing responsibilities. The model does not consider practical storage issues such as supercapacitor leakage and self-discharge, or cycle exhaustion in rechargeable batteries. When nodes change state a message is sent to the energy model informing it of the current drawn by the previous state and how long the node spent in that state. State specific current draw figures and minimum state elapsed times for the QualNet 5.2 [201] simulated motes are shown in Table 3.3, receive and transmit current draw figures coming from direct measurement by resistor shunt as show in Table 3.1 .

Historic average incident radiation data for $41^{\circ} 19^{\prime} 24^{\prime \prime} \mathrm{S}, 174^{\circ} 46^{\prime} 12^{\prime \prime} \mathrm{E}$ (Wellington, New Zealand) were obtained from public records [202] and a solar panel selected from commonly available components [203] with a form factor slightly larger than the $81.90 \mathrm{~mm} \times 32.50 \mathrm{~mm}$ Advanticsys CM5000 motes used in the study. 
Charge available for storage in a given period $s$ seconds starting at time $T$ is:

$$
Q=\sum_{t=T}^{T+s} M I N\left\{I_{p},\left(\frac{\lambda_{t} \times l_{p} \times w_{p} \times \eta_{p}}{V_{p}}\right)\right\} \times \eta_{c}
$$

where $\lambda_{t}$ is the incident radiation at time $t, \eta_{p}$ and $\eta_{c}$ are the efficiencies of the solar panel and charging circuit respectively, $I_{p}$ and $V_{p}$ are peak current and peak voltage of the solar panel of length $l_{p}$ and width $w_{p}$ respectively. Solar panel specifications are as shown in Table 3.2.

Table 3.2: Solar Panel Specifications

\begin{tabular}{ccccc}
\hline Model & Efficiency & Peak V & Peak C & Dimensions \\
\hline SZGD6161 & $16.5 \%$ & $2.2 \mathrm{~V}$ & $92 \mathrm{~mA}$ & $61 \mathrm{~mm} \times 61 \mathrm{~mm}$ \\
\hline
\end{tabular}

Table 3.3: Advanticsys CM5000 Simulation Parameters

\begin{tabular}{lr}
\hline Current Draw (mA) & \\
Sleep $^{1}$ & 0.0001 \\
Idle Listen & 18.400 \\
Receive & 19.216 \\
Transmit & 19.116 \\
Transceiver Warm Up & 18.400 \\
\hline Minimum Elapsed Times (ms) & \\
Send & 2.5 \\
Receive & 2.5 \\
Transceiver Warm Up & 3.0 \\
\hline
\end{tabular}

${ }^{1}$ From manufacturers specification sheet [197] 


\subsection{Impact on Existing Research}

Transmission Power Control (TPC) schemes for WSNs have been of interest for some time with many active research projects [204]. In 2014, Transmission Power Control-based Opportunistic Routing (TCOR) was proposed [205]. Amongst TCOR's assumptions is that transmitting at a low power level consumes less energy than transmitting at higher power. Similarly, MACRO [187], one of the geographic forwarding mechanisms discussed in Section 2.3.2, makes the assumption that reducing transmit power uses less energy. Yet for the devices examined here, this is clearly not the case.

A wider reading of the literature suggests formulating energy models can be error prone, a number of myths having emerged in stated or implicit assumptions about how WSN devices work. As the most power hungry component, most of these invalid assumptions concern the radio transceiver. In this chapter, two commercially available WSN devices were examined and it was observed that a number of frequently repeated WSN energy consumption assumptions are invalid for those devices.

It should be noted that references to research relying on these "busted myths" are given solely to demonstrate that had that research been undertaken on the devices used here, one or more basic assumptions would have been invalid. The original authors, however, may have had other devices in mind where their assumptions would indeed hold. Nonetheless, a taxonomy of inaccuracies to avoid when adopting WSN energy models for simulationscan be identified:

Sending a packet is not the most energy hungry operation: Keeping a device's transceiver energised for idle listening is far more expensive.

Turning on the transceiver is not instantaneous: There is a significant warm up time that can consume as much energy as sending a packet.

Decreasing transmit power does not use less energy: If the transceiver is already energized, it draws the same current regardless of transmit power.

Generic energy consumption models do not fit all devices: Significant differences in energy consumption are observed between similar devices from different manufacturers.

It should also be noted that whilst reducing transmit power does not save energy at the individual device level, the corresponding reduction in transmission 
range may realise reduced energy consumption across the extended network if doing so reduces unnecessary processing and re-transmission of overheard packets. However, as we have shown, idle listening dominates energy consumption in WSN devices so reducing transmission range may not save a significant amount of energy. In this and all WSN scenarios, it is clear that realistic, device-specific energy models are fundamental to meaningful simulations.

\subsection{Summary}

It has been shown here that evaluating current draw via voltage drop across a resistor shunt is just as effective a measurement technique as more complex methods. Further, a list of inaccuracies to avoid when adopting WSN energy models for simulations has been identified in Section 3.8. Most significantly for algorithms presented in subsequent chapters, evidence based power consumption and energy harvesting model have been created.

Measurements taken during the assessment inform the algorithms presented in subsequent chapters by demonstrating that sending data at high transmit powers does not consume any additional energy compared to sending the same data at low transmit powers. This insight is of particular significance to SOFIE, the rapid forwarding algorithm presented in Chapter 5, insofar as SOFIE broadcasts all data at maximum transmit power to gain maximum propagation distance; this approach being show here to place SOFIE at no disadvantage to competing algorithms that chose to broadcast at lower transmit powers. 


\section{Chapter 4}

\section{Equitable Sleep Coverage}

Time sensitive geospatial occurrences, rare events that are both ephemeral and transitory, pose a challenge to wireless sensor networks (WSNs) deployed for their detection. Maximising detection probability (likelihood the event is detected) and minimising detection delay (time taken for notification to reach a network sink) implies sufficient nodes need to be active at all times to both maintain initial sensing coverage and provide a route to the network sink delayed only by transmission overhead. Energy saving pauses during transmission such as those implemented in media access control (MAC) protocols featuring receiver initiated transceiver de-activation [24] or demand wake-up [25] could be regarded as having too great an impact on detection delay. In this study, detection delay is minimised by having an always-on, always connected communication route to the network sink at the cost of significant energy consumption through idle listening [2].

When full coverage is ensured, the connectivity comes naturally if we assume that the communication range is at least twice the sensing range [154]. The emergence of low power wide area network (LPWAN) technologies may help ensure connectivity, but bandwidth is very low and further study is needed to evaluate their ability to meet detection delay requirements [206].

Establishing and maintaining sensing coverage in WSNs for rare events can be regarded as ensuring every point in the sensing area is covered by at least one sensor node at all times whilst maintaining energy efficiency [26]. WSNs with a sufficiently dense overpopulation of sensing nodes can self-organise such that a given node is in a position to power down if a subset of its neighbours are willing to take temporary responsibility for its sensing area. In battery powered WSNs, 
this duty-cycling extends the operational lifetime of a subset of deployed nodes and in doing so can both extend the period during which the network maintains its initial coverage and the period during which there is at least some coverage [27].

This chapter introduces the Equitable Sleep Coverage Algorithm for Rare Geospatial Occurrences (ESCARGO) for unreliable network protocols designed to simultaneously address both coverage and connectivity requirements for critical rare event sensing. The efficacy of the algorithm is assessed through simulation and live deployment in randomly placed and optimally distributed networks of identical sensing nodes with both uniform and non-uniform sensing ranges, powered by batteries as well as energy harvesting. Further analysis is undertaken to determine charging efficiency required to maintain rare event sensing coverage in connected networks powered by energy harvested using photovoltaic technology. Small form-factor solar cells are chosen to both minimise costs and match the dimensions of commercially available sensing nodes.

\subsection{Coverage Preservation Algorithm}

We assume a two dimensional rectangular distribution of location and sensing area aware nodes. Non uniform sensing ranges [145] and arbitrary polygonal sensing areas [207] have been studied and ESCARGO can be modified for either case without compromising its efficacy; for the majority of this study, individual node sensing areas are assumed identical and exactly circular, and the node communication range is at least twice that of the sensing range to preserve network connectivity [154]. Underlying network protocol reliability is not assumed.

\subsubsection{Overview}

Nodes are in one of four states: sponsored where sensing responsibility is delegated to at least one other node, sponsoring where they are taking responsibility for the sensing area of one or more neighbouring nodes, seeking where they are actively attempting to find neighbouring nodes willing to sponsor them, and passive where they are un-sponsored, not seeking sponsorship, and are not sponsoring any of their neighbours. A state transition diagram for the algorithm is shown in Figure 4.1. 


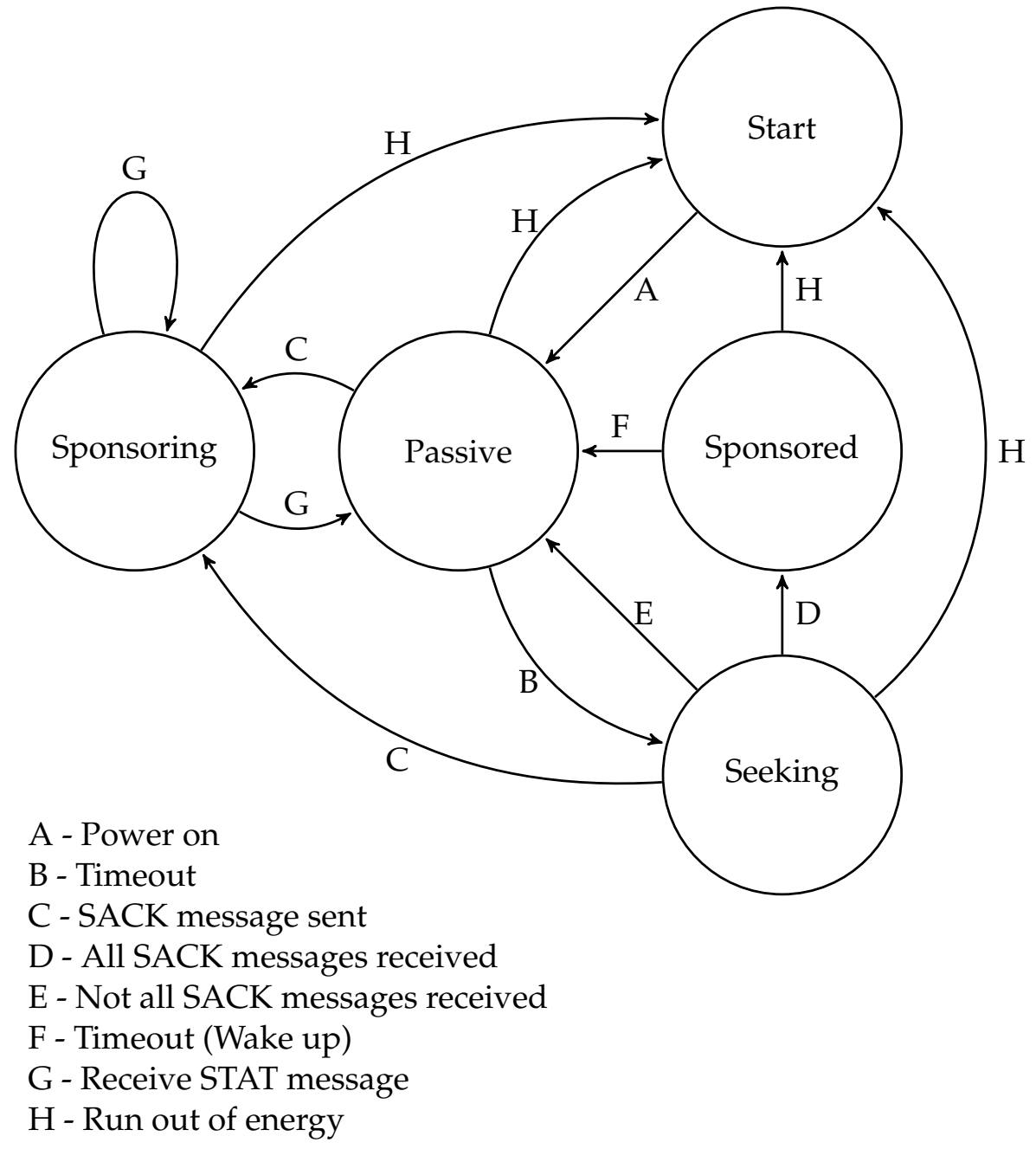

Figure 4.1: ESCARGO State Transitions

All nodes start simultaneously and enter the passive state, broadcasting periodic STATus (STAT) messages containing their position and cumulative runtime statistics. STAT messages are broadcast at random intervals between $200 \mathrm{~ms}$ and $300 \mathrm{~ms}$ for a random period between $2 s$ and $3 s$. During this start-up period nodes listen for STAT messages from other nodes and determine who their sensing neighbours are. Two nodes are deemed to be sensing neighbours if they are separated by a distance less than or equal to their common sensing range. Pseudocode for passive state is given in Algorithm 3.

A node is eligible to enter sponsored state if and only if it determines its sensing area is fully contained by the union set of some combination of its passive, seeking or sponsoring neighbours' sensing areas. Combinations of neighbour- 


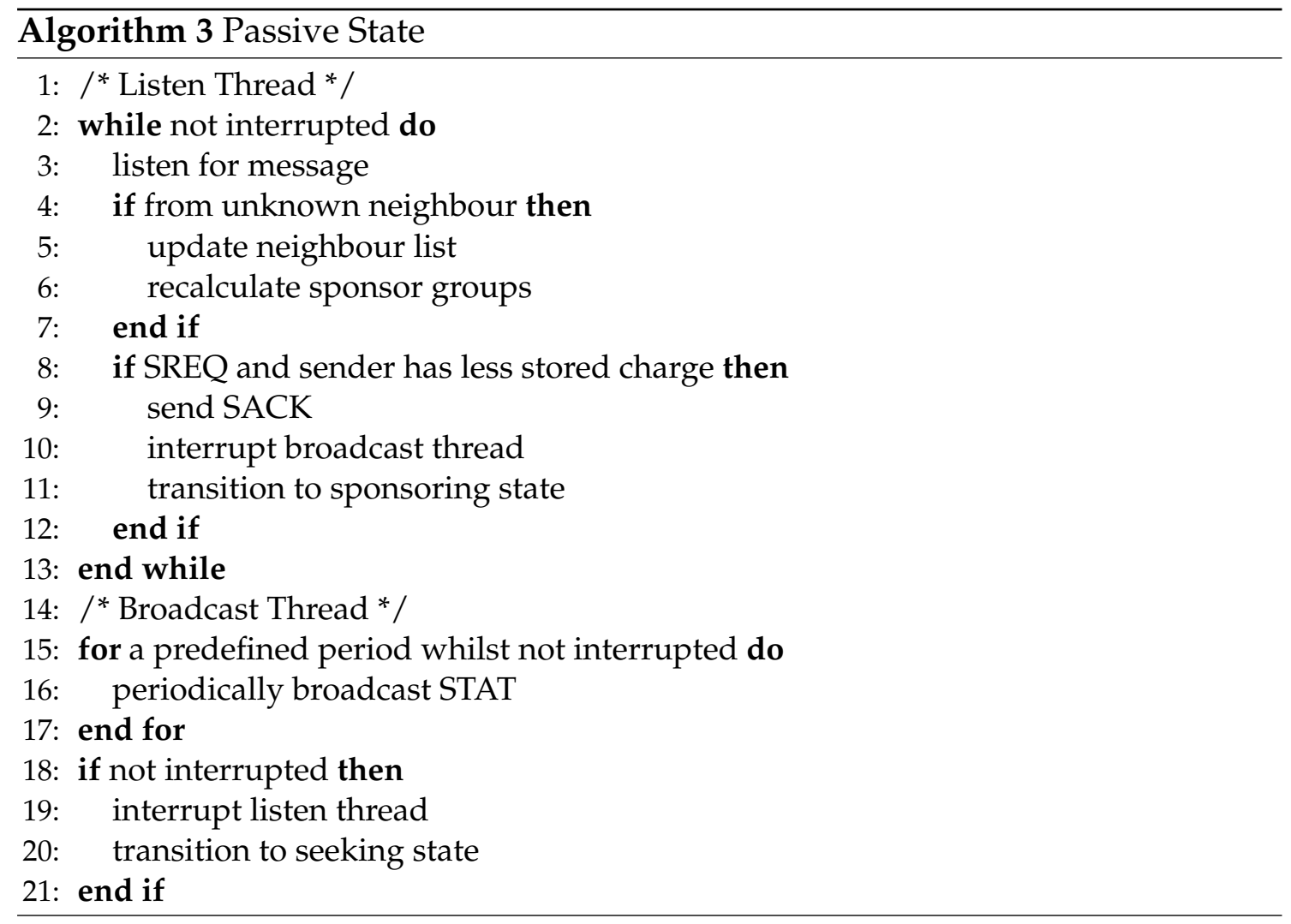

ing nodes that could sponsor a given node are known as sponsor groups. Sponsor group membership is determined by geometric calculations taking into consideration the boundary of the sensing area as detailed in Section 4.1.2. Nodes adjust their sponsor group lists each time a STAT from a previously unknown sensing neighbour is received.

Nodes that deem themselves eligible for sponsorship cycle through their sponsor groups in a round-robin fashion, seeking sponsors starting with the group after the previous sponsor group. For each sponsor group selected, a Sponsor REQuest (SREQ) message is sent to each node in the group. Nodes in receipt of an SREQ add the requesting node to their sponsored list and return a Sponsorship ACKnowledgement (SACK) message. Once a node has agreed to be a sponsor it will no longer attempt to gain its own sponsors until it is notified by the nodes it is sponsoring that they no longer require assistance. Pseudocode for the seeking state is given in Algorithm 8.

When a seeking node wishing to be sponsored has received a SACK from each of the nodes it sent an SREQ to, it enters sponsored state for a predetermined period. In sponsored state, nodes enter a low energy mode where all sensors and the radio transceiver are powered down. 


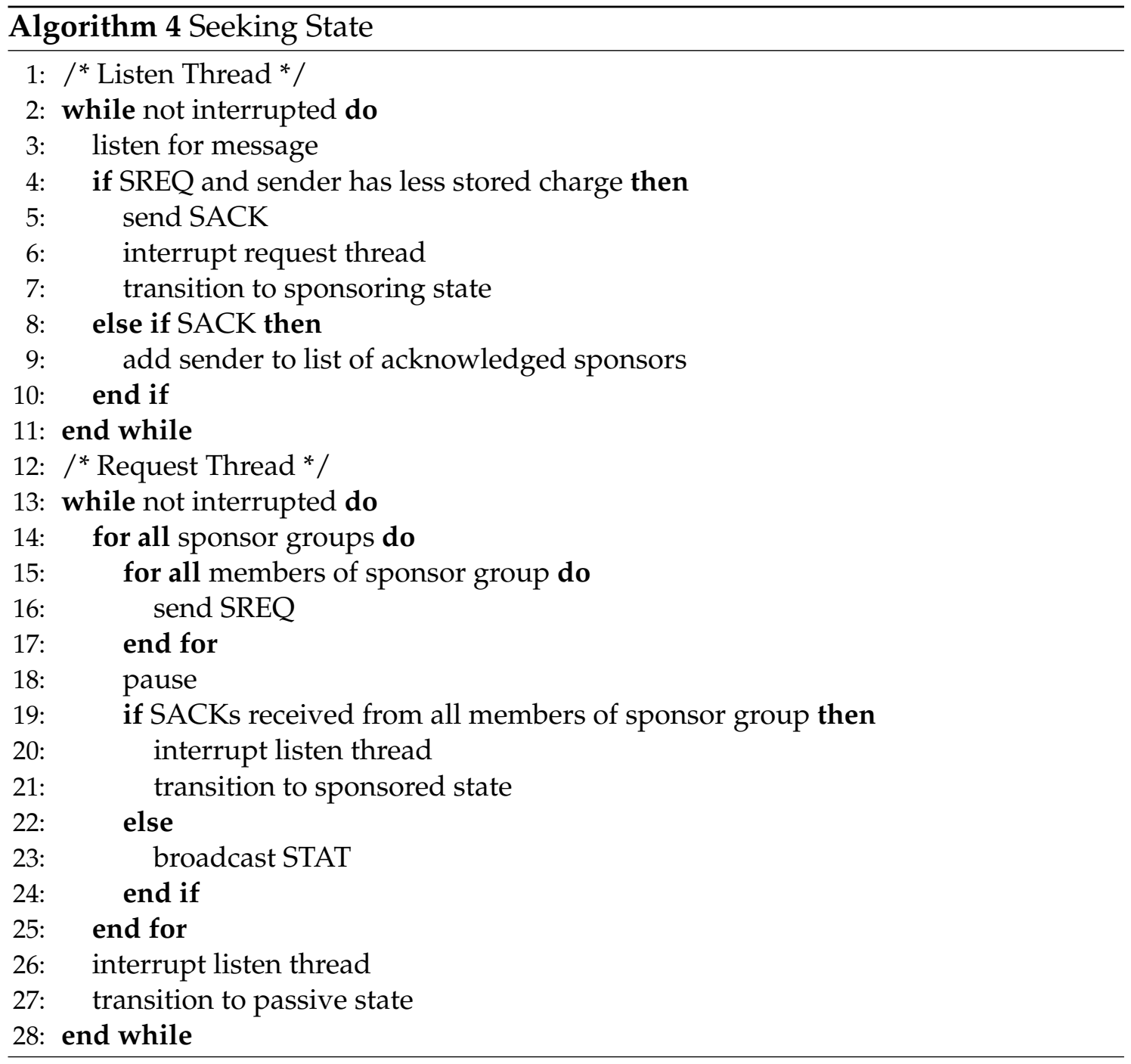

Pseudocode for sponsored state is trivial so not shown.

If fewer SACKs are received than SREQs sent, the requesting node reverts to passive state and broadcasts STATs (cf: Algorithm 3). Neighbouring nodes that had already agreed to be sponsors remove the requesting node from their sponsored lists on receipt of any of these STATs. On wake-up from a period of sponsorship, nodes similarly broadcast STATs and their sponsoring nodes adjust their sponsored lists accordingly. Pseudocode for sponsoring state is given in Algorithm 5.

SREQs contain the stored charge of the requesting node. Potential sponsor nodes will respond to the SREQ with a SACK if and only if the sponsoring node has more stored charge than the requester. Whilst waiting for SACKs, a seeking node that receives an SREQ from a node with less stored charge than itself will 


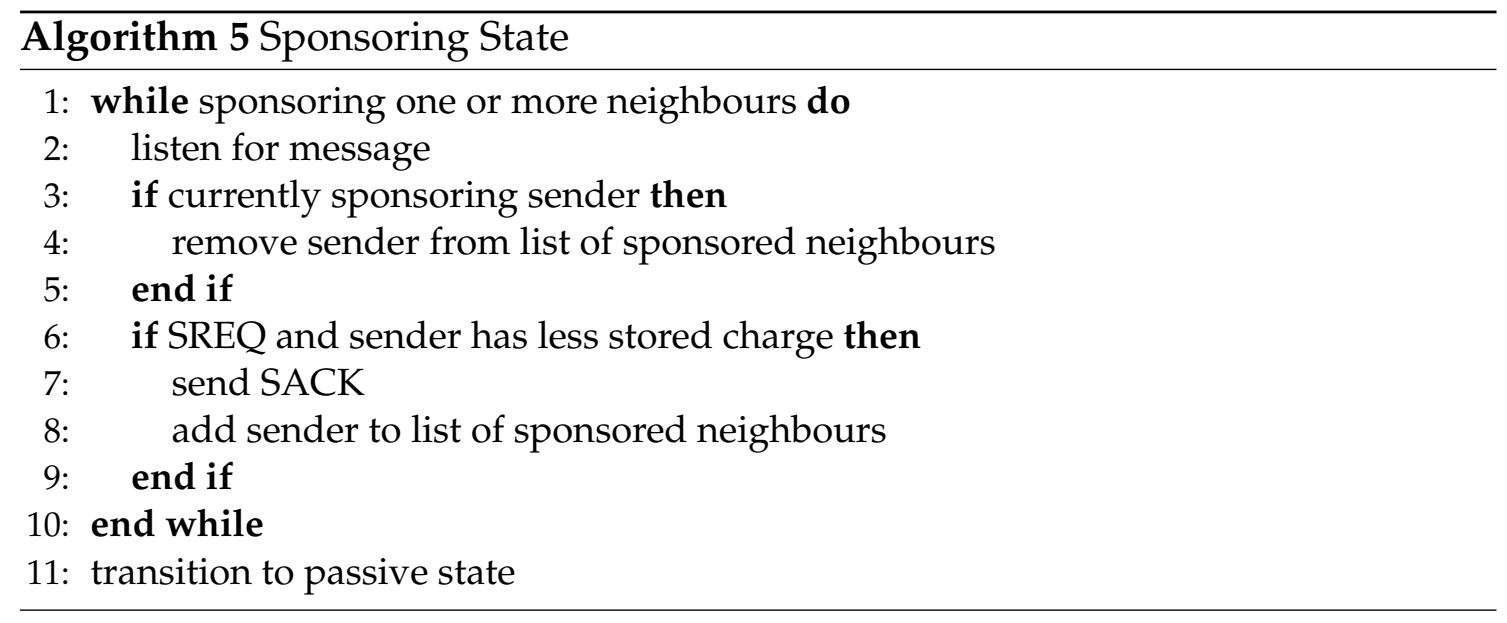

cease waiting for any remaining inbound SACKs, send a SACK of its own to the requesting node and enter sponsoring state.

\subsubsection{Sleep Eligibility}

When a node goes to sleep it takes no part in the sensing activities or communication links of the WSN until it wakes up again. To maintain sensing coverage in the WSN, the entire sensing area a sleeping node was responsible for must be covered by one or more neighbouring nodes. Only when a node is confident its sensing area is covered by a set of awake neighbours does it become sleep eligible.

Wang et al. [154] performed geometric analysis of the relationship between connectivity and coverage in networks made up of nodes with uniform sensing and transmission ranges.

Theorem $1 A$ convex region $A$ is $K$-covered by a set of neighbours if: (1) there exist in A intersection points between neighbours or between neighbours and A's boundary; (2) all intersection points between neighbours are at least $K$-covered; and (3) all intersection points between any neighbour and A's boundary are at least $k$-covered.

This is the $k$-coverage eligibility rule. ESCARGO only requires nodes to exhibit 1-coverage eligibility, an example being given in Figure 4.2a, where the node with the highlighted sensing area is eligible to enter sleep mode as its sensing area is completely covered by neighbouring nodes. Note that the node with sensing area marked $A$, whilst overlapping slightly with the sensing area of the eligible node (shown by the darkest circle) is not required to act as a sponsor for the eligible node. 


\subsubsection{Node Placement}

This paper considers random node distributions and an ideal, planned placement of nodes based on a repeating and overlapping pattern of three nodes. The three nodes' sensing area perimeters, being of equal length, intersect the vertices of an equilateral triangle of side $2 \sqrt{3} R_{s}$ where $R_{s}$ is the node sensing range, and intersect with each other at the midpoint of the triangle's sides, as shown in Figure $4.2 \mathrm{~b}$. This placement being identical to one where each node is separated by $\sqrt{3} R_{s}$ for each neighbour [208].

When a third node is added at the intersection of the three existing nodes sensing ranges, the new node is 1-coverage eligible with a single sponsor group made up of the original three nodes, as illustrated in Figure 4.2c. Repeating this pattern using existing nodes as members of new groups leads to a uniform coverage where each node is at least 2-coverage eligible, having no less than two sponsor groups.

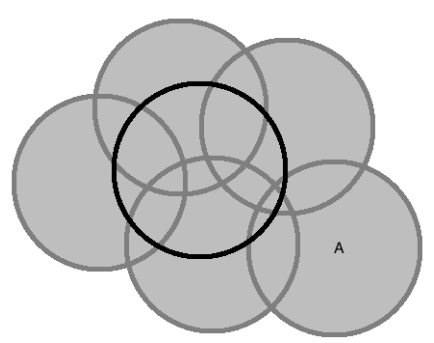

(a)

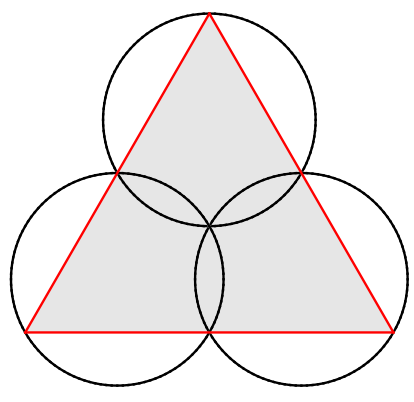

(b)

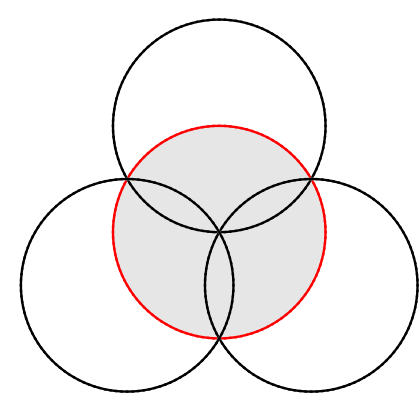

(c)

Figure 4.2: (a) Example of 1-coverage eligibility in random distribution; node with sensing area marked $A$ is not required to provide coverage for the node with sensing area bounded by the darkest circle. (b) Node placement based on equilateral triangle of side $2 \sqrt{3} R_{s}$ where $R_{s}$ is the node sensing range. (c) Node placed at the intersection of existing nodes becomes 1-coverage eligible.

Sponsor group membership for nodes whose sensing area and those of its immediate neighbours are entirely contained within the network sensing area is illustrated by the example placement shown in Figure 4.3a. Node 2 is the only one with sponsor groups, shown in Table 4.1.

Note that a number of other potential sponsor groups exist, but they form redundant super sets of the three minimal sponsor groups. E.g. the potential sponsor group containing nodes $1,6,7$, and 4 can be simplified by a sponsor group containing nodes 1,6 , and 7. The placement in Figure 4.3a is sub optimal 
as six of the seven nodes have no sponsor groups and will be unable to power down by entering sponsored state as less that $100 \%$ of their sensing areas are covered by other nodes.

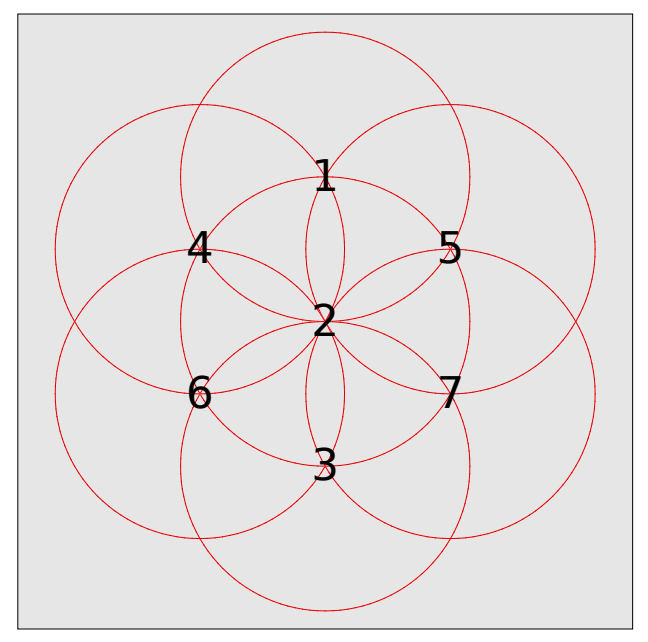

(a) 7 nodes

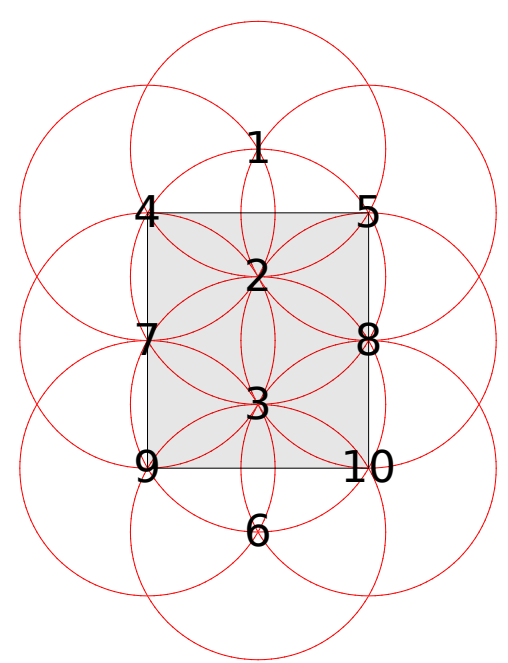

(b) 10 nodes

Figure 4.3: Placement of nodes relative to sensing area, node identifiers shown, sensing area shaded.

Table 4.1: Sponsor Groups for Nodes in Figure 4.3a

\begin{tabular}{cl}
\hline Node ID & Sponsor Groups \\
\hline 2 & {$[1,6,7][3,4,5][4,5,6,7]$} \\
\hline
\end{tabular}

Figure $4.3 \mathrm{~b}$ shows a placement of ten nodes over a small sensing area. Only nodes 2 and 3 are within the bounds of the sensing area, the remaining nodes being located outside the sensing area or on the boundary itself. In this configuration, all nodes have at least one sponsor group as those on or beyond the sensing area boundary do not need their entire sensing area sponsored, just the subsection coincident with the network sensing area. Node 4 can be sponsored either by Node 2 alone, or by a combination of nodes 1 and 7. Sponsor groups for nodes in Figure $4.3 \mathrm{~b}$ are shown in Table 4.2.

In random distributions, it is difficult to predict how many nodes will be at least 1-covered. However, for sufficiently dense distributions the probability of each node having at least one sponsor group approaches 1. Figure 4.4 charts the ratio of nodes with at least one sponsor group against density as a multiplier of that found in an ideal, planned placement exemplified by the one in Figure 4.3b; 
Table 4.2: Sponsor Groups for Nodes in Figure 4.3b

\begin{tabular}{cl}
\hline Node ID & Sponsor Groups \\
\hline 1 & {$[4,5][2]$} \\
2 & {$[4,5,7,8][1,3,5,7][1,3,4,8][1,7,8][3,4,5]$} \\
3 & {$[2,6,7,10][2,6,8,9][7,8,9,10][6,7,8][2,9,10]$} \\
4 & {$[1,7][2]$} \\
5 & {$[1,8][2]$} \\
6 & {$[9,10][3]$} \\
7 & {$[2,3][2,9][3,4]$} \\
8 & {$[2,10][2,3][3,5]$} \\
9 & {$[6,7][3]$} \\
10 & {$[6,8][3]$} \\
\hline
\end{tabular}

a density of at least two-and-a-half times the ideal placement being required to allow the possibility of all nodes being at least 1-covered.

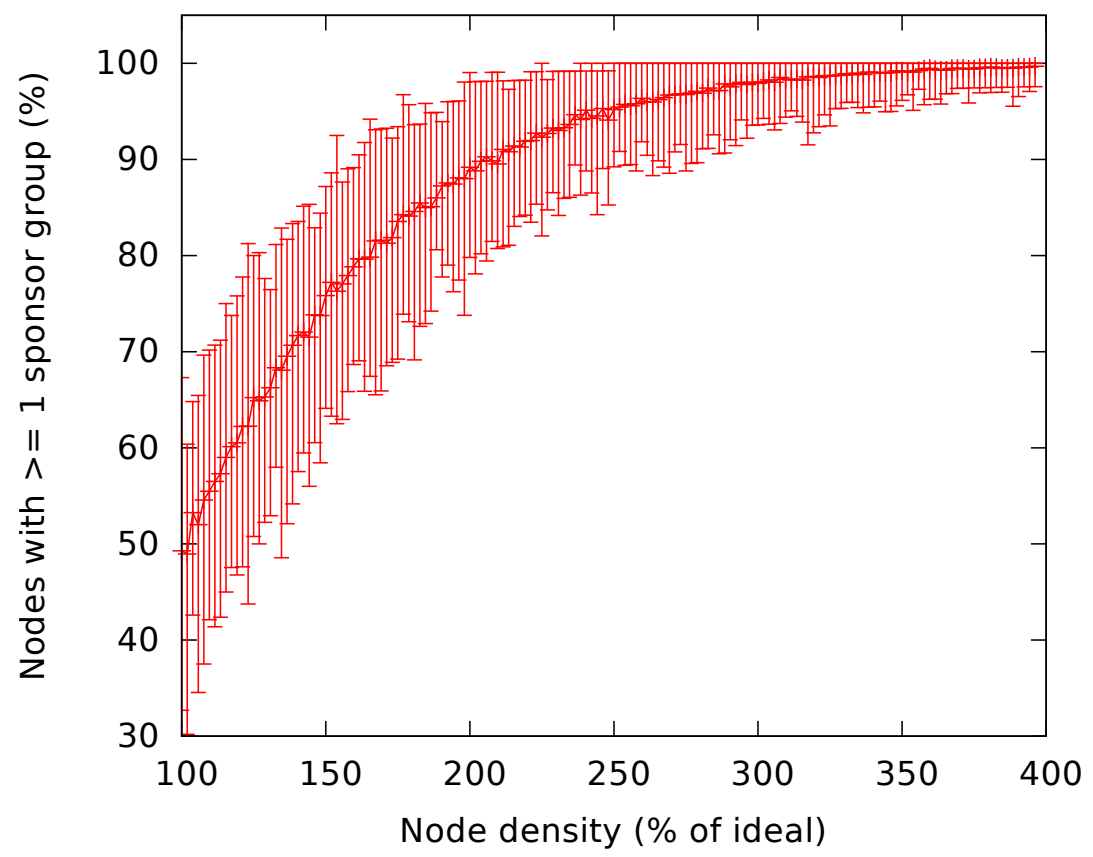

Figure 4.4: Nodes with at least one sponsor group by deployment density. 


\subsection{Evaluation}

In the ESCARGO simulation undertaken, changes in node state that could lead to an alteration in coverage trigger a recalculation over the network sensing area. Nodes entering sponsored state do not impact coverage. Nodes running out of energy whilst acting as a sponsor will decrease coverage but if a currently sleeping node runs out of energy, coverage will be preserved as its sensing area is being covered by other sponsor nodes. Two metrics are of interest, namely, coverage and stored charge.

\subsubsection{Energy Model \& Simulation Parameters}

ESCARGO is implemented in QualNet 5.2 [201] with all nodes configured as IEEE 802.15.4 compliant motes running as non-beaconing full-function devices with CSMA/CA parameters as shown in Table 4.3. Power consumption and solar energy harvesting is modeled as described in Section 3.7.

Table 4.3: IEEE 802.15.4 CSMA/CA Parameters

\begin{tabular}{ll}
\hline Min Backoff Exponent & 3 \\
Max Backoff Exponent & 6 \\
Max CSMA Backoffs & 4 \\
Max Frame Retries & 3 \\
Turnaround Time & 12 symbol periods \\
Unit Backoff Period & 20 symbol periods \\
ACK Wait Duration & 650 symbol periods \\
\hline
\end{tabular}

ESCARGO requires transmission range to be at least twice sensing range; the antenna height and transmission power of the Advanticsys CM5000 motes used in the simulation have been set to achieve a radio range of $94.8 \mathrm{~m}$. Sensing range is set to $40 \mathrm{~m}$. An initial charge of $4800 \mathrm{mAh}$ is given to each node, equivalent to two Duracell NiMH AA rechargeable batteries [209].

All simulations start at 00:00 on January 1 . Note that the solar radiation data are for the southern hemisphere, as such, the simulations start approximately one month before the height of summer. 


\subsubsection{Algorithm Efficacy}

Using the 10-node planned placement shown in Figure 4.3b, a full calendar year of operation was simulated for four operational scenarios:

1. Battery only - no energy harvesting, no synchronised sleep scheduling.

2. Battery power with ESCARGO.

3. Energy harvesting replaces battery power but ESCARGO is disabled.

4. ESCARGO and energy harvesting combined.

Mean stored charge for all nodes during the first month of operation is shown in Figure 4.5.

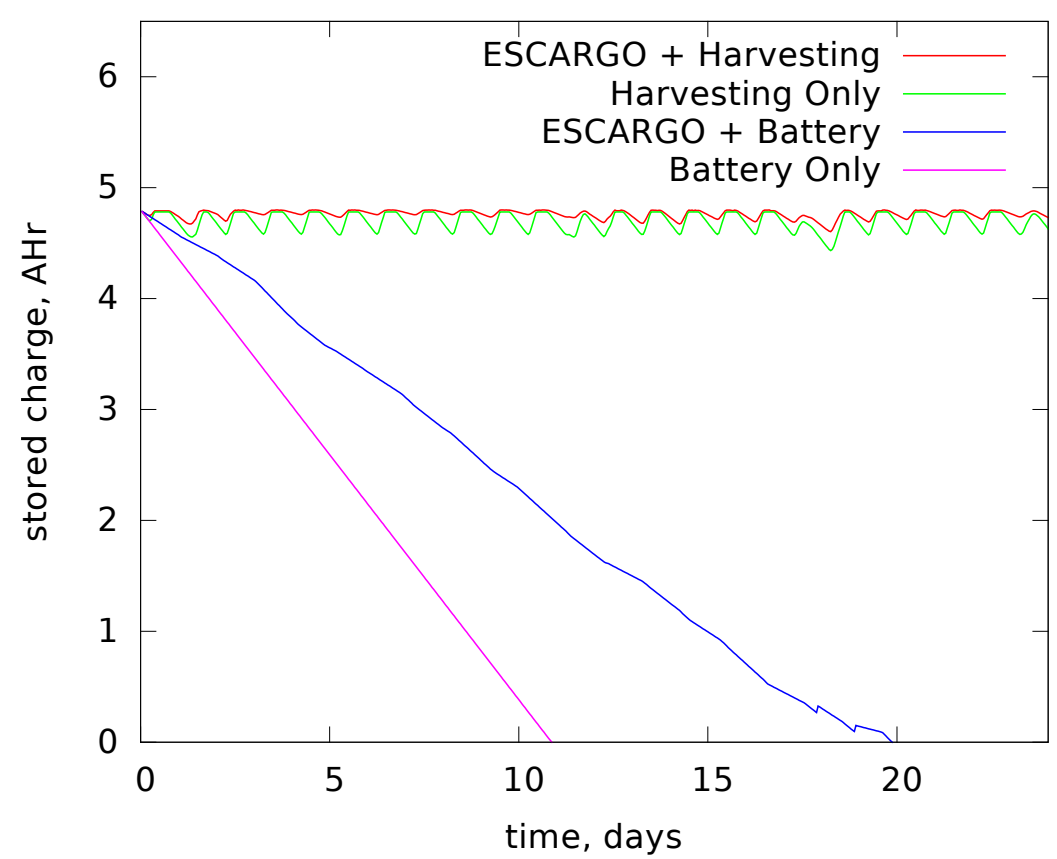

Figure 4.5: Mean stored charge during first month of operation.

On battery power alone, stored charge depletes entirely in around 11 days at which point all coverage is lost. Adding ESCARGO to battery power extends network lifetime significantly. However, original coverage is not maintained for the entire period. As nodes' stored charge become exhausted, they die and coverage is compromised. Figure 4.6 charts the percentage of original coverage plus percentage of sleeping and expired nodes over the same time period as Figure 4.5. 


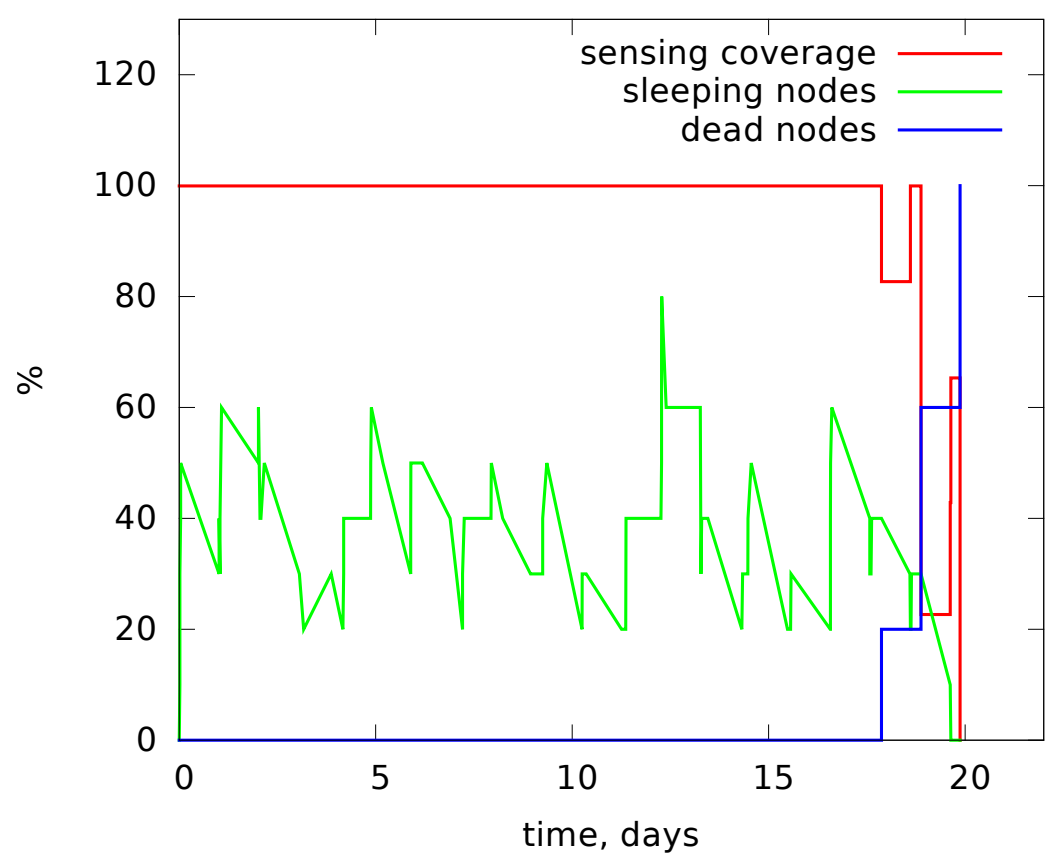

Figure 4.6: Sensing coverage \& node state, battery power with ESCARGO.

Original coverage is maintained until around day 17 when the first nodes die. Coverage is restored briefly as sponsored nodes return to passive state then tails of quickly as further nodes die.

During the first month of operation (the height of summer) adding ESCARGO to energy harvesting maintains a higher mean stored charge, as shown in Figure 4.5 , but the practical benefits are negligible as more energy is available for harvesting than can be stored and used. During the winter months, when less energy is available, a harvesting only solution exhibits a sharp degradation in average stored charge as more energy is used each day than can be replenished. Combining ESCARGO with energy harvesting results in improved mean stored charge. Figure 4.7 shows mean stored charge for both scenarios.

For the harvesting only network in Figure 4.7, no nodes have the opportunity to sleep and coverage is compromised whenever stored charge is exhausted. Coverage is restored when dead nodes re-activate after harvesting additional energy, only to be compromised again on subsequent exhaustion. Coverage oscillates between $100 \%$ and $0 \%$ as all nodes in the network die and re-activate simultaneously. For the harvesting and ESCARGO network in Figure 4.7, no nodes die, a high proportion of nodes are asleep at any given time, and initial sensing coverage is maintained indefinitely. 


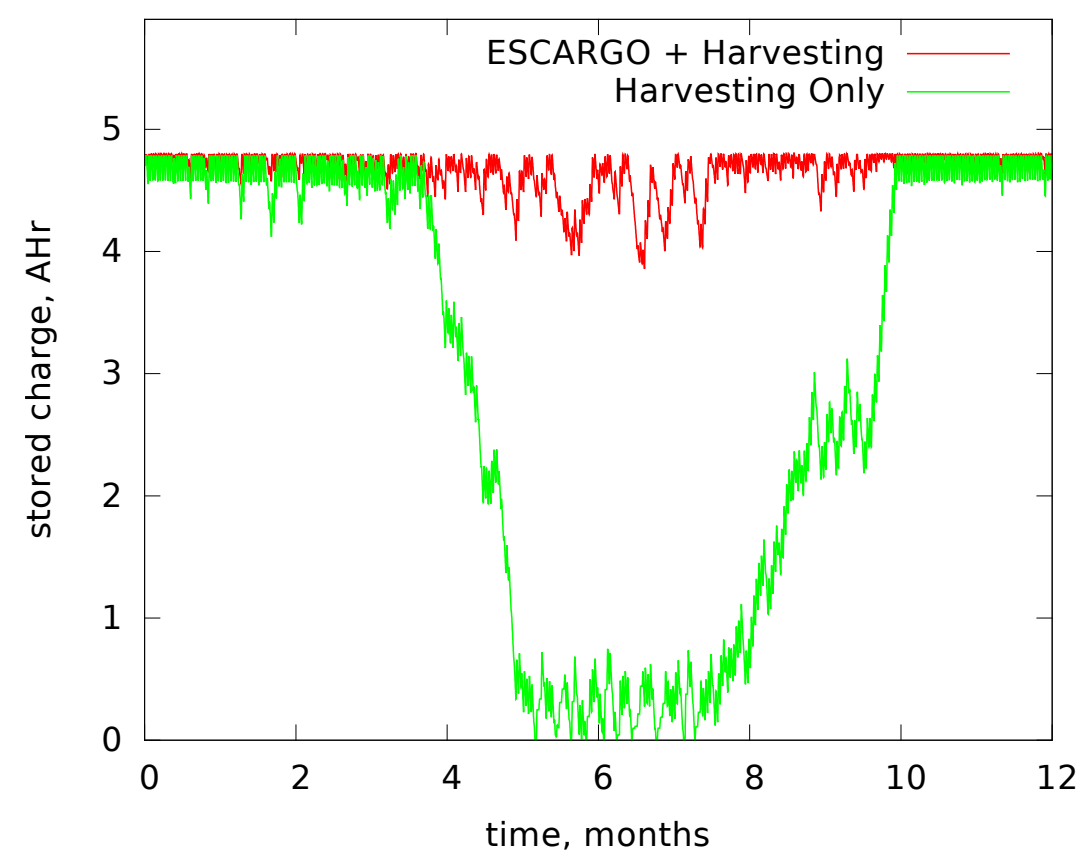

Figure 4.7: Mean stored charge over one calendar year, 70\% efficient charging.

\subsubsection{Required Charging Efficiency}

Khosropour et al. [29] propose a low power charging circuit with $90 \%$ efficiency and reference other works representing similar systems with charging efficiencies of $67 \%$ [210], 70\% [211], and 86\% [212]. Running simulations based on the planned 10-node distribution in Figure $4.3 \mathrm{~b}$ and varying the charging efficiency $\eta_{c}$ in Eqn. (3.1) show a charging efficiency of $55 \%$ is sufficient to maintain mean stored charge close to $50 \%$ of original. Figure 4.8 shows mean stored charge over a full calendar year for charging efficiencies between $50 \%$ and $70 \%$.

For planned placements, ESCARGO assures equitable discharge and re-charge for all nodes in the WSN, hence any charging efficiency that realises a non-zero average stored charge across all nodes will ensure $100 \%$ of original coverage is maintained. Figure 4.8 shows a charging efficiency of $50 \%$ is insufficient to maintain sensing coverage throughout the year. Simulations configured for this charging efficiency exhibit multiple occasions where a node runs out of energy but coverage is maintained as the node in question was being sponsored at the time, whilst on one occasion stored charge in the network drops to zero and all nodes die, coverage only being restored when a sufficient number of nodes have harvested additional energy. The point where coverage is compromised coincides with the point in Figure 4.8 where average stored charge drops to zero. 


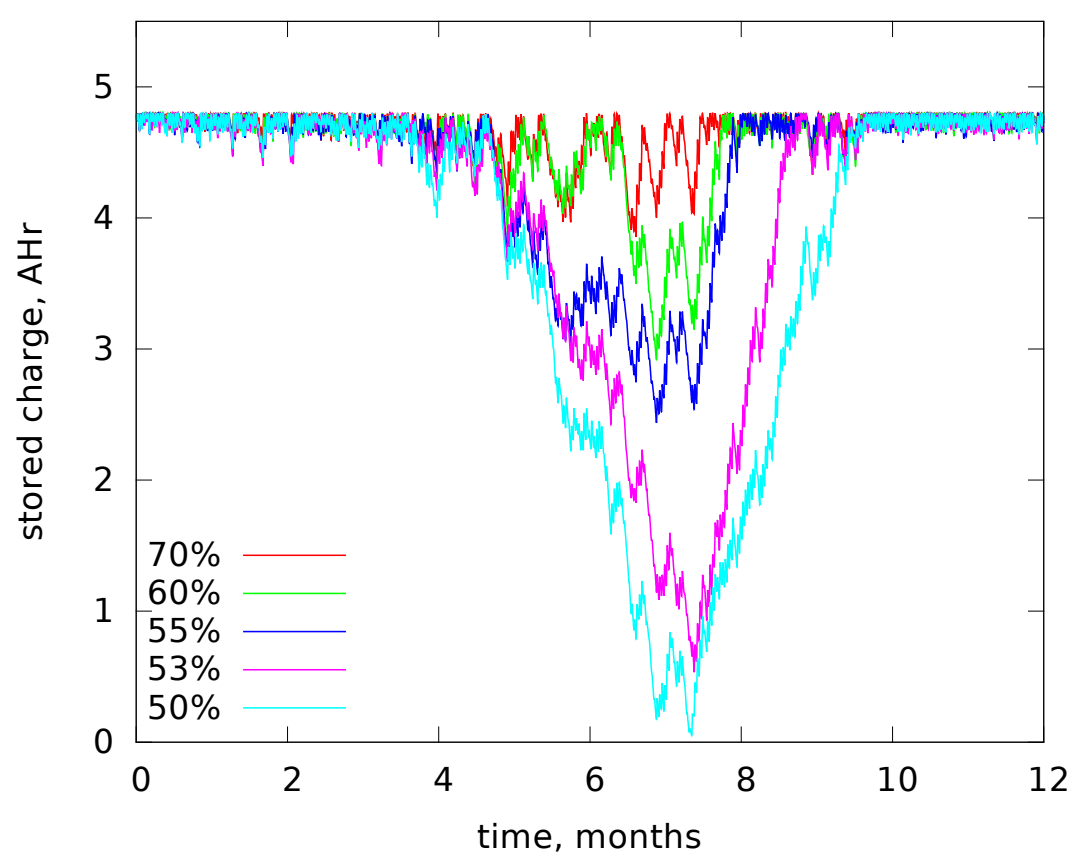

Figure 4.8: Mean stored charge over time by charging efficiency.

\subsubsection{Comparative Study}

RACP [27], discussed in Section 2.2.3, has similar aims to ESCARGO. Both algorithms alternate sponsoring duties between nodes in a non-time synchronised, collaborative fashion to extend the operational life of deployed WSNs. However, RACP makes no attempt to equalise node sleep times and in certain deployment configurations some nodes become hot spots where they find themselves taking an unequal share of the sponsorship responsibility, leading to premature energy exhaustion and a reduction in sensing coverage.

This implementation of RACP differs in one significant respect to the published algorithm; sensing area boundary awareness has been incorporated. Without this addition, nodes at the outer edges of the network never get the opportunity to sleep, resulting in an ever shrinking coverage area as nodes exhaust their energy reserves. As RACP is not designed for energy harvesting networks, comparison with ESCARGO is undertaken using a battery energy model.

Random placements of thirty eight nodes with $R_{s}=7$ in a $25 \mathrm{~m} \times 25 \mathrm{~m}$ sensing area (as shown in Figure 4.9a) where generated, those not providing all nodes with at least one sponsor group were rejected until one hundred random placements were available. Simulations with distinct random seeds were executed one hundred times for each placement, first with ESCARGO, then with RACP, then 


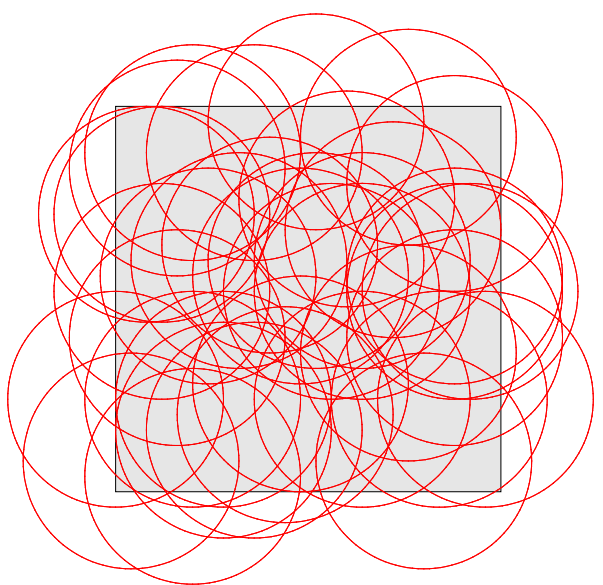

(a)

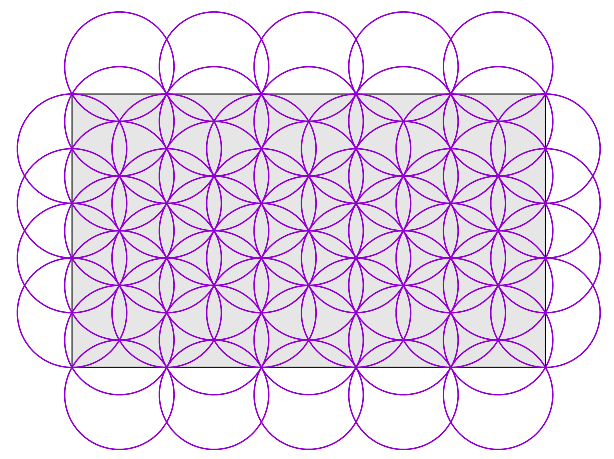

(b)

Figure 4.9: Placements for comparative study and evaluation of physical implementation: (a) 38 nodes, random, $R_{s}=7$; sensing areas shaded. (b) 59 nodes, planned, $R_{s}=40$.

with neither algorithm active. The metric of interest being the time at which $100 \%$ initial (perfect) coverage is lost.

Figure 4.10 charts the mean time perfect sensing coverage was lost by scenario, clearly showing RACP performs slightly less well than doing nothing at all; the additional energy cost of sending and receiving coordination messages draining the resources of nodes that found themselves hot spots and, as such, unable to sleep very often, if at all. Figure 4.10 also shows ESCARGO extends the time the network can provide perfect coverage by as much as $50 \%$.

ESCARGO outperforms RACP by such a wide margin because RACP is unable to equalise node sleep times. Figure 4.11a charts the sleep time from one simulation run for the 38 node random placement in Figure 4.9a for RACP; several nodes are able to asleep up to almost half the time, others hardly ever. Perfect coverage is lost as soon as the node allowed to sleep least often exhausts its energy supplies and shuts down. The last node to run out of energy, doing so, many hours after the first.

Figure $4.11 \mathrm{~b}$ charts the same simulation configuration for ESCARGO; all 38 nodes sleep for a similar amount of time, narrowing the gap between node deaths, and preserving perfect coverage far longer than RACP. It should be noted that the extent to which ESACRGO maintains perfect coverage beyond RACP is directly proportional to the minimum sleep percentages nodes exhibit when running each algorithm, in this instance, around 30\%. 


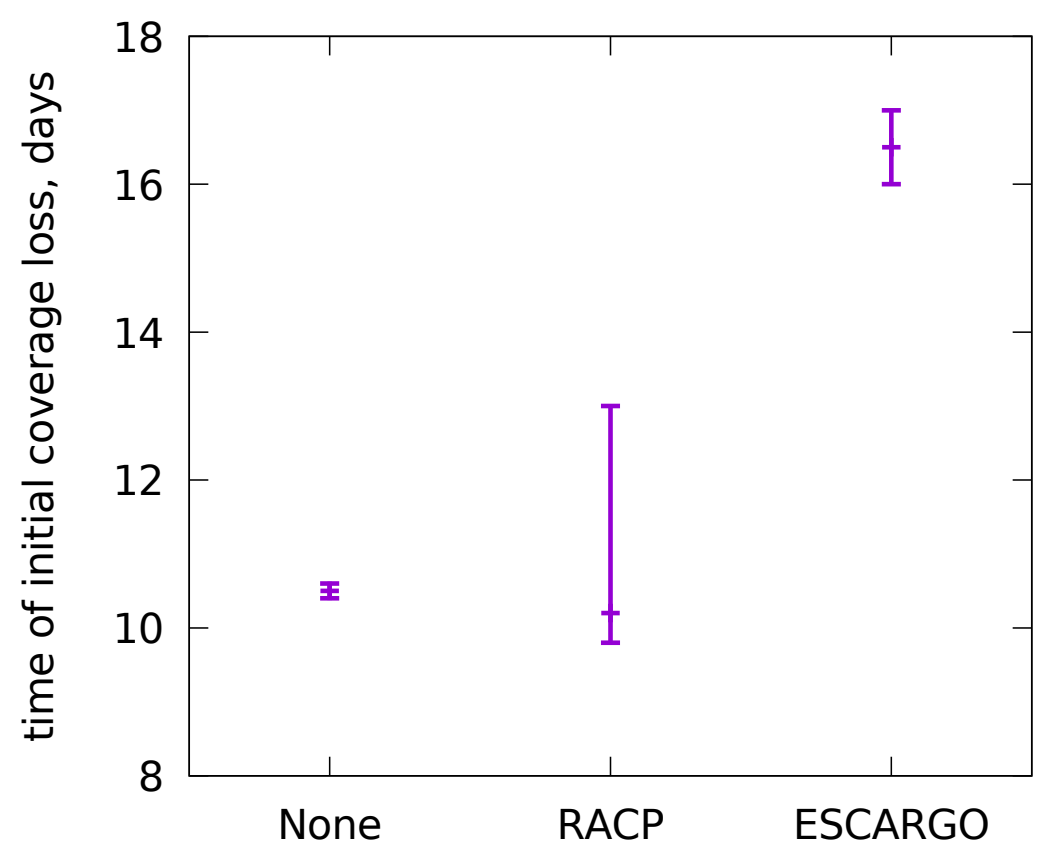

Figure 4.10: Time of initial coverage loss by algorithm. Minimum, mean maximum from 100 randomly seeded simulations. 38 nodes, random placement (Figure 4.9 a), battery power.

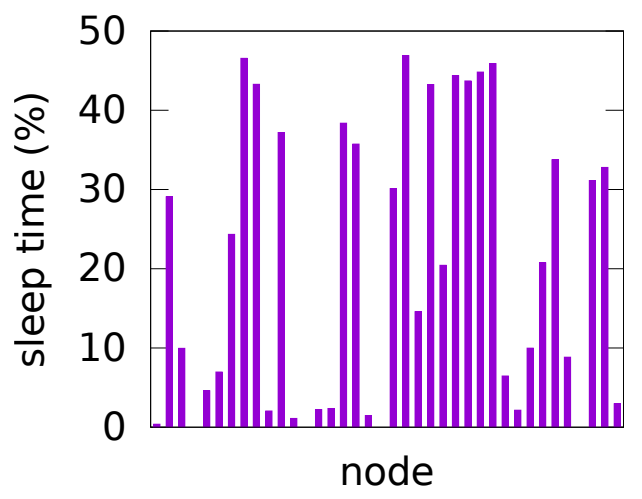

(a) RACP

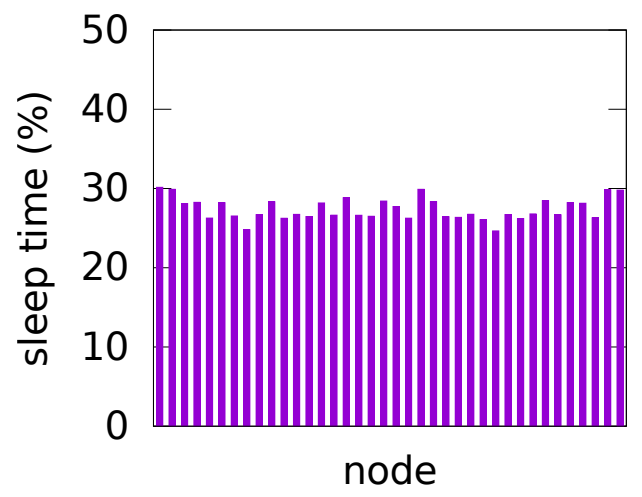

(b) ESCARGO

Figure 4.11: Percentage sleep time by algorithm; 38 nodes, random placement (Figure 4.9a), battery power. 


\subsubsection{Non-Uniform Sensing Ranges}

In many situations sensing ranges are arbitrarily assigned and are to a large extend notional. The Advanticsys CM5000 devices used in this study have on-board temperature, humidity, and light sensors, but they are point sensors to the extent that they measure attributes of the environment at their location. It is convenient, however, to accept that the temperature at point $\mathrm{A}$ is a reasonable indicator of the temperature at point $\mathrm{B}$ if the distance $\mathrm{AB}$ is small. That small distance can re regarded as the sensing range of the devices, $R_{s}$.

To accommodate sensing devices with non-uniform sensing ranges, ESCARGO can be trivially modified to include device sensing ranges in STAT messages; the distinct values for $R_{s}$ are then used in the geometric calculations employed to determine which nodes are neighbours and sponsor group membership, c.f. Section 4.1.2, and note that Theorem 1 is independent of sensing range and clearly holds for both uniform as well as non-uniform sensing ranges.

Figure 4.12 a shows a 52 node dense random deployment where nodes have a variety of sensing ranges, the largest being twice that of the smallest. Figure $4.12 \mathrm{~b}$ shows stored charge for all nodes in this configuration over one calendar year. Comparison to Figure 4.7 indicates ESCARGO is just as effective for non-uniform sensing ranges as it is in the uniform case.

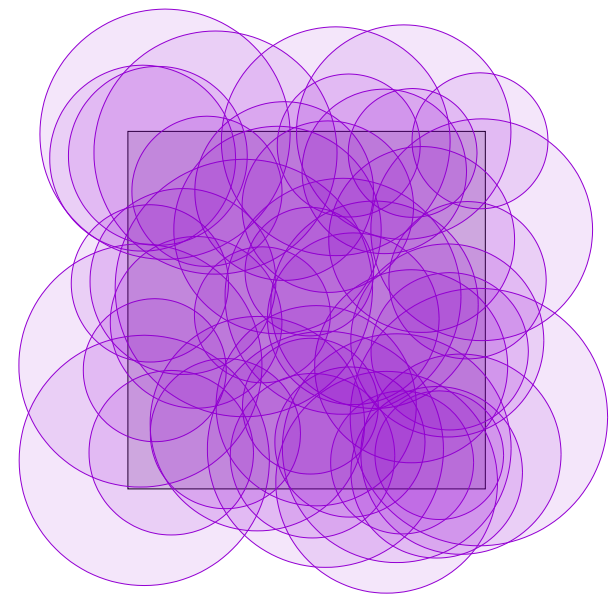

(a)

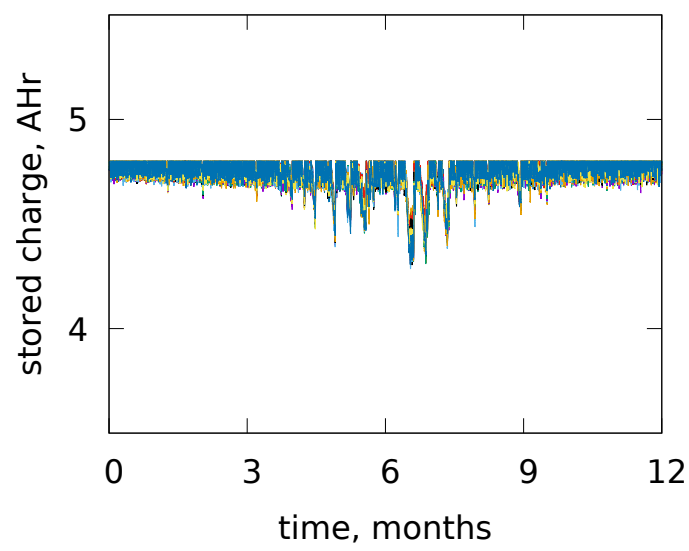

(b)

Figure 4.12: Random distribution of 52 nodes in a 100x100m sensing area, $R_{s}$ in the range $18 \mathrm{~m}$ to $36 \mathrm{~m}, R_{c}=80 \mathrm{~m}$ : (a) Node distribution (b) Stored charge over one calendar year, $53 \%$ charging efficiency. 


\subsubsection{Implementation \& Simulated Energy Harvesting}

A TinyOS/NesC [104] implementation of ESCARGO for the Advanticsys CM5000 motes modeled in the finite state simulations in Sections 4.2.2, 4.2.3 \& 4.2.4 was produced, and a testbed capable of supporting up to sixty nodes constructed. The testbed consists of a single Linux desktop computer with sixty five USB ports made available through a hub-and-spoke arrangement of six, thirteen port USB hubs. The CM5000 motes are powered by their USB connection and, if built in debug node, send logging information to the server via the USB cable. Figure 4.13 shows an aerial view of the test bed.

On start-up, nodes request individual and system wide configuration from a base station. Configuration messages received in response to this request include specification of the solar cell to use, charging efficiency to adopt, geographic coordinates, and a time synchronisation token. Note that whilst the test bed requires time synchronisation, ESCARGO itself does not. Nodes do not enter the initial passive phase of ESCARGO until they have received their configuration.

Once configured and ESCARGO activated, each time a node changes state it sends to the base station a message containing the percentage (\%) of time it has spent asleep and an indication of the stored charge remaining. If a mote reboots for some reason, the last indication of stored charge will be sent back to it by the base station enabling it to carry on as if it were able to calculate the stored charge.

Figure 4.14 plots sleep time for each node in the initial hour of a test using the 38 randomly placed nodes in Figure 4.9a; over time, the sleep time converges to approximately $30 \%$, entirely consistent with the results seen in Figure $4.11 \mathrm{~b}$. Figure 4.15 charts stored charge over the same time period, during which no energy is available for harvesting; the downward trend is consistent across all nodes, sections where the plots appear parallel to the $\mathrm{x}$-axis are periods when the node in question was in sponsored state, consuming almost no energy. Figure 4.16 shows the sleep time for a node that has restarted for some reason rapidly converging back to the network mean value.

Energy harvesting is simulated in the test bed by periodically broadcasting a message containing an indication of the currently incident tilted radiation. On receipt of such a message, test bed motes store the radiation value for rapidly scheduled calculations of the stored charge using Eqn. (3.1). Included in the incident radiation message is a time multiplier indicating how much faster than real time the device broadcasting the messages is changing the radiation values. 


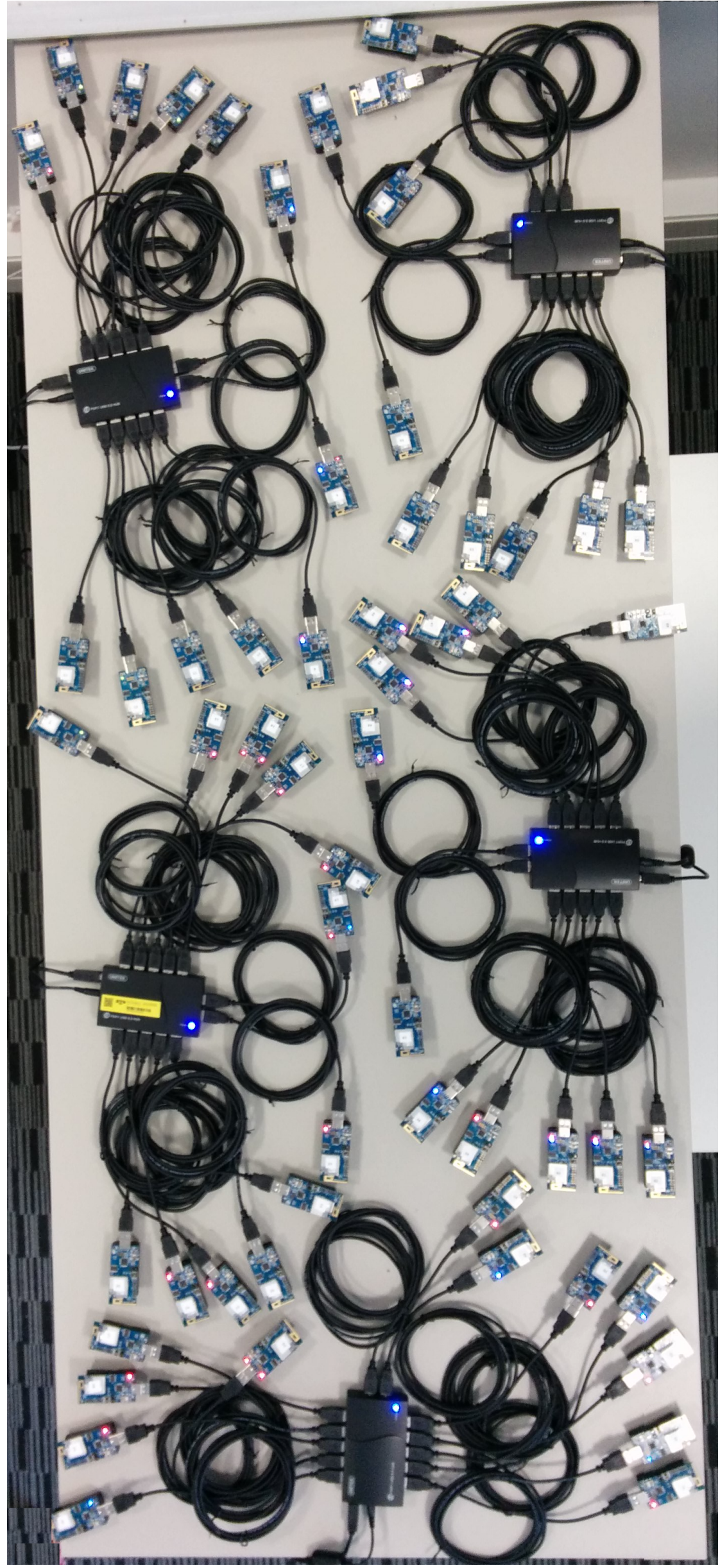

Figure 4.13: Test environment with 59 nodes from Figure 4.9b 


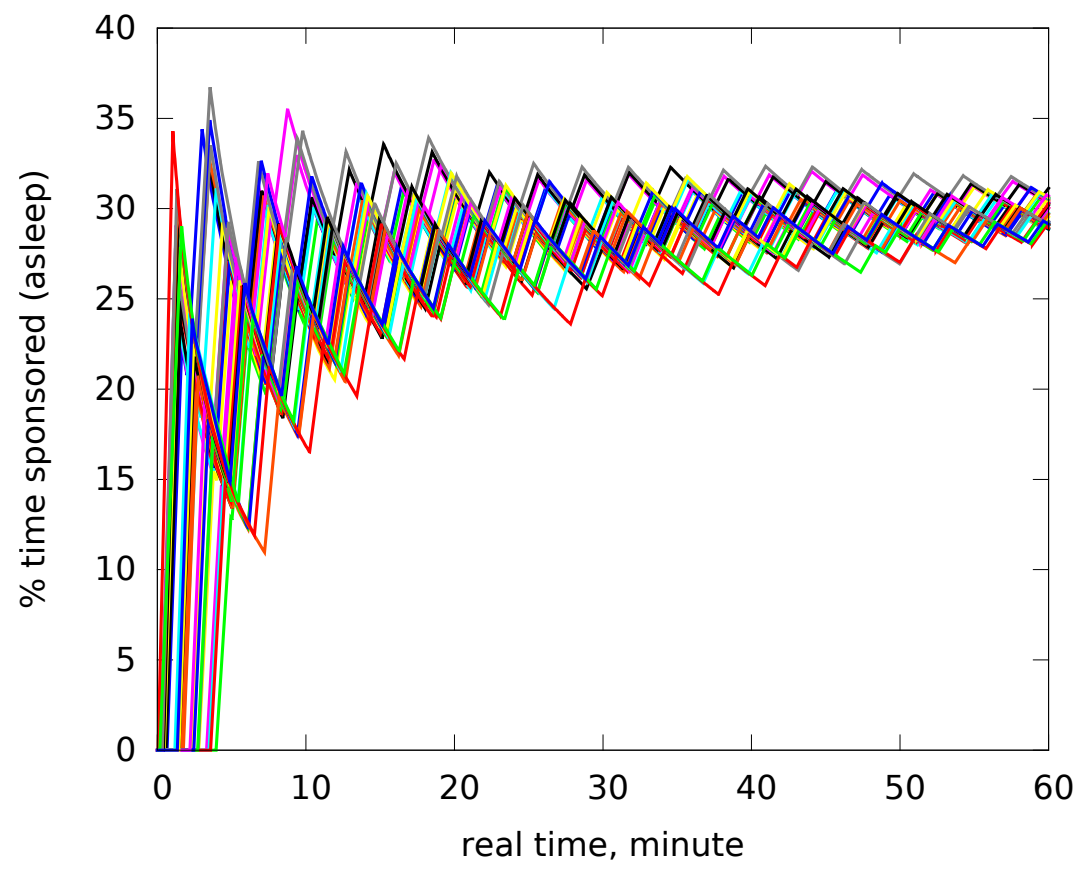

Figure 4.14: Implementation: Sleep times converging rapidly after initialisation.

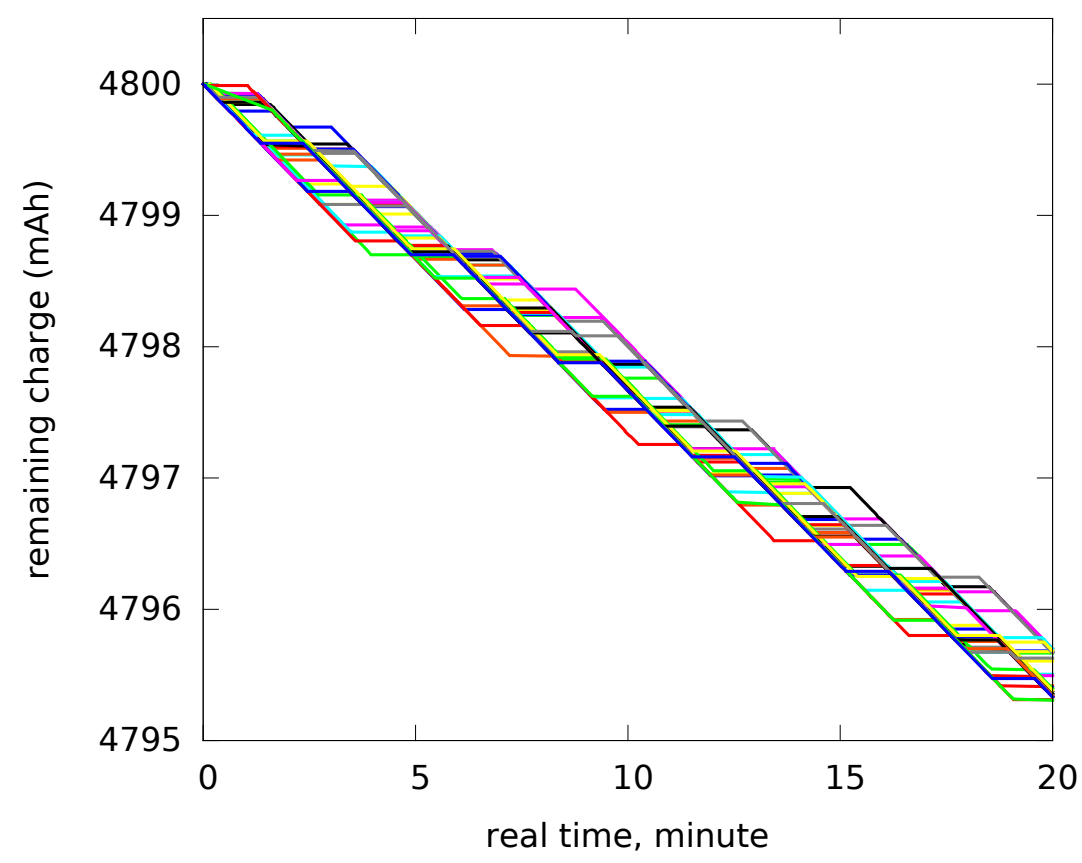

Figure 4.15: Stored charge is equalised in initial non harvesting period; horizontal sections indicate sponsored (sleeping) nodes. 


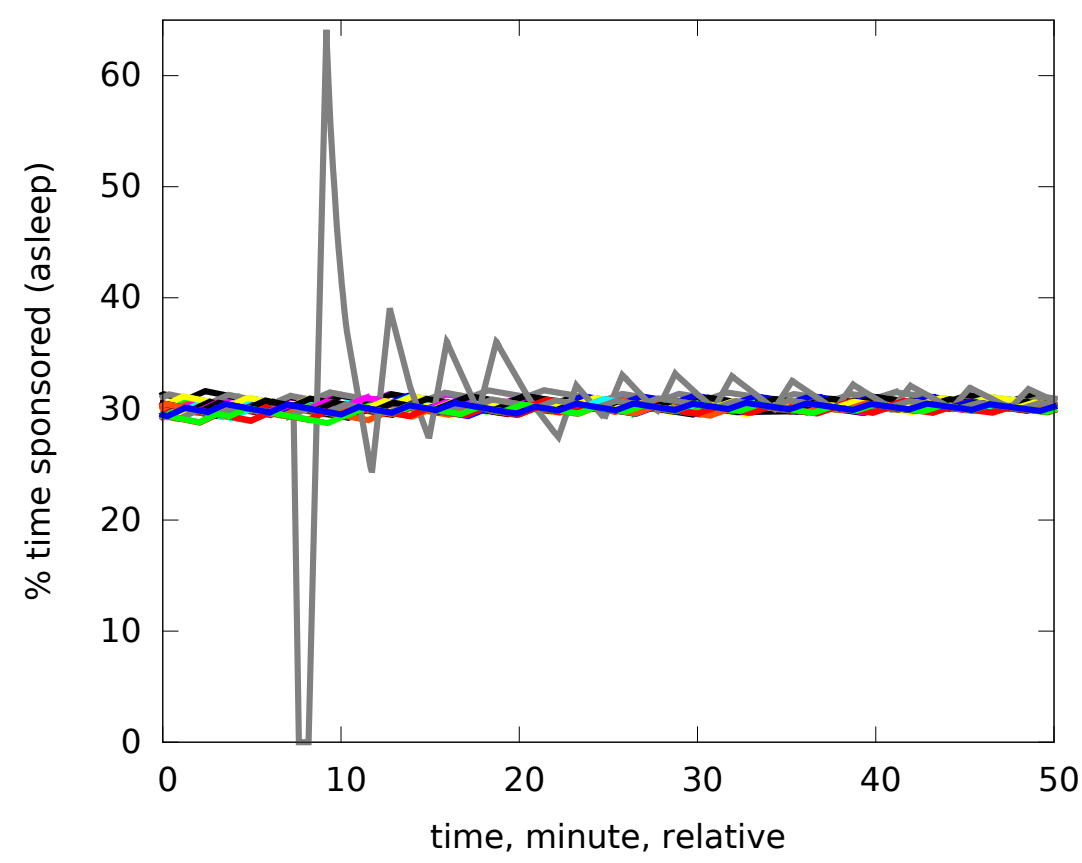

Figure 4.16: If a node restarts, its sleep time rapidly converges back to the mean after staying awake long enough to ensure any nodes it was sponsoring have woken up.

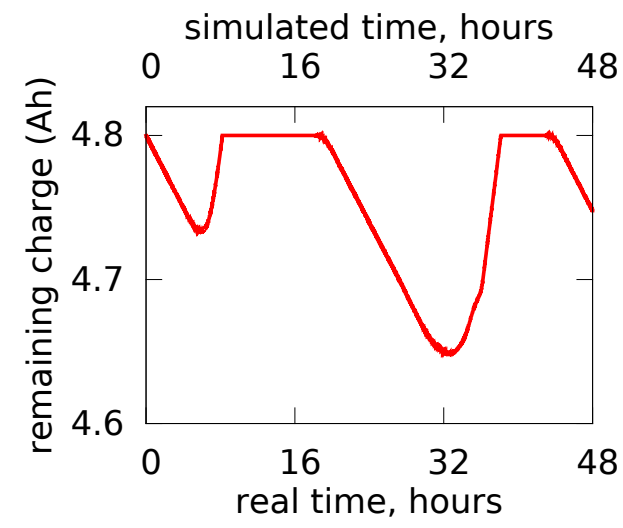

(a)

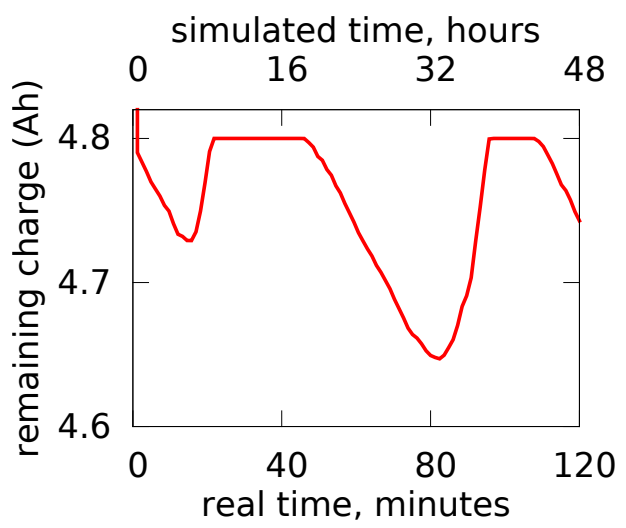

(b)

Figure 4.17: Stored charge over first 48 hours of operation: (a) Real-time, no multiplier. (b) Multiplier $=24$, one day simulated in one hour. 
This mechanism allows the test bed to consume a years worth of solar radiation in a little over twenty four hours if the multiplier is set to 360 .

Figure 4.17a shows mean stored charge for the 10-node placement in Figure $4.3 \mathrm{~b}$ over a 48 -hour time period, starting 00:00, January 1, where the multiplier is set to 1, i.e. simulated time matches real time. As dawn breaks, energy becomes available for harvesting and stored charge increases until it hits the maximum storage capacity (4800 mAh) where it stays until the Sun starts to set. Stored charge drops again until the Sun comes up on January 2 when it rises once more to the maximum storable value. Note that as the simulated energy harvesting starts at midnight, the first local minima in the chart is less extreme than the second as each mote was active without the possibility of harvesting additional energy for several more hours. Similarly, the local plateaus are of different length as the motes reach full charge later in the day on January 2.

Figure 4.17a shows mean stored charge for the same 10-node placement from Figure $4.3 \mathrm{~b}$, this time with a multiplier of 24 , allowing the same two days worth of radiation to be consumed in two hours. Fewer data points are captured, yet the curves follow the same trends with local maxima and minima of identical value occurring in the same places.

A multiplier of 360 consumes a full year of radiation in one day; Figure 4.18 charts stored charge for the 38 randomly placed nodes in Figure 4.9a in the test bed configured in this fashion. From the start of the year until around the end April, nodes are able to completely replenish their charge during daylight hours. As winter approaches, stored charge decreases steadily until the spring when an increasing surplus of energy becomes available, maximum stored charge being restored around mid September.

\subsubsection{Simulation and Implementation Equivalence}

Whilst the implementation and simulation code bases share algorithmic components, the Qualnet and TinyOS runtime environments have no common features so care must be taken implementing environment specific versions of ESCARGO.

Figure $4.11 \mathrm{~b}$ shows percentage sleeptime for a network of simulated ESCARGO nodes, the mean sleeptime clearly being in the order of $28 \%$. Similarly, Figure 4.14 shows sleeptime for a network of physical ESCARGO nodes converging on a value in the order of $28 \%$. 


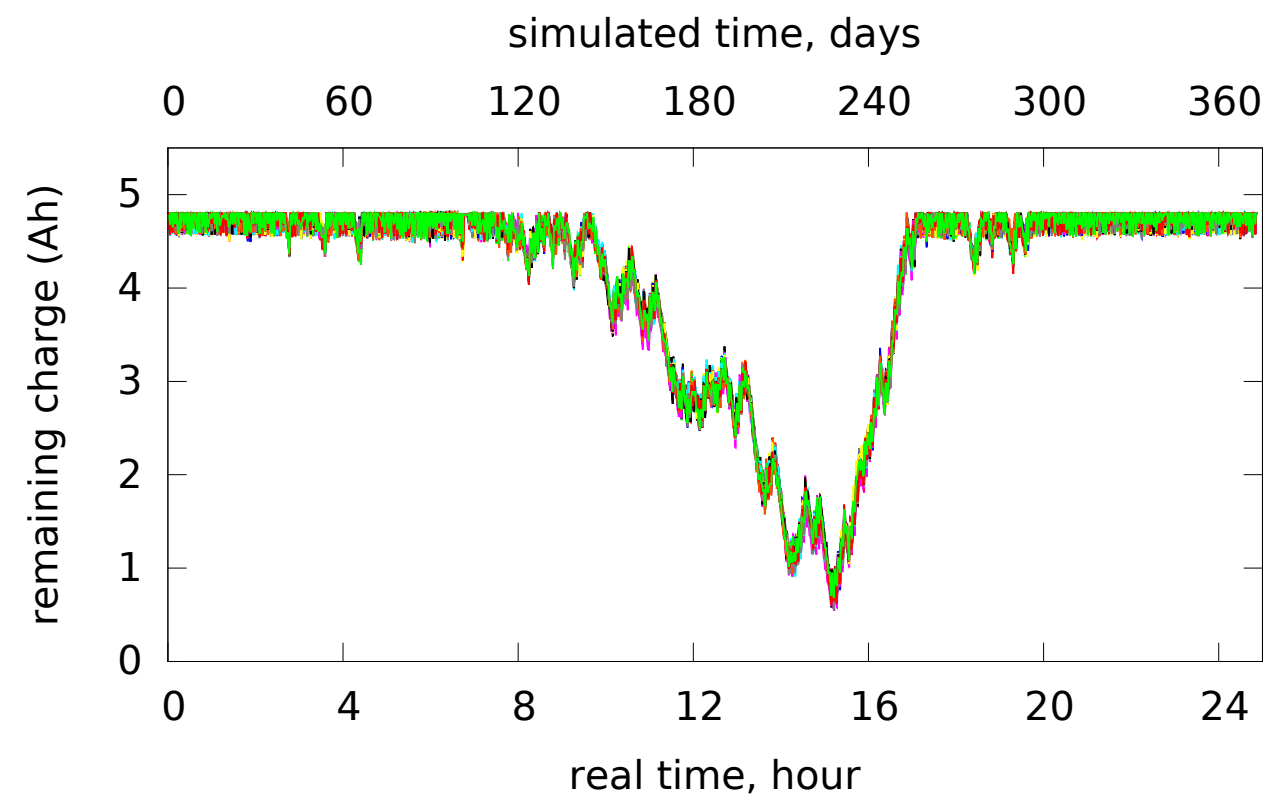

Figure 4.18: Stored charge over one simulated calendar year, 38 nodes, random placement (Figure 4.9a), 70\% charging efficiency.

\subsection{Summary}

Evaluation of the sleep scheduling algorithm ESCARGO, in non-time-synchronized rare event monitoring WSNs powered by energy harvested from small formfactor solar panels, indicates initial sensing coverage in low density planned node placements can be maintained indefinitely with charging efficiencies within the capability of existing power management systems. ESCARGO is also shown capable of approximately doubling the lifetime of the same WSN when nodes are battery-powered and to outperform an alternate algorithm with related aims.

ESCARGO is further shown to be effective in sufficiently dense random distributions where 1-coverage is guaranteed for all nodes. However, random distributions give rise to situations where a fraction of nodes have a large number of sponsor groups, space for storing which may not be available on the modest capability devices this thesis is targeting. 


\section{Chapter 5}

\section{Swift Opportunistic Forwarding}

To minimise detection delay, forwarding protocols must minimise algorithmic delays. The simplest mechanism to achieve this is to re-broadcast event notifications immediately, where in practice this immediacy is bounded only by the modest processing power of the sensing devices, and the standards based CSMA/CA algorithms they use.

Assume a two dimensional distribution of location aware wireless sensor nodes, each of which knows the location of all network sinks. Sufficient nodes are placed within the sensing field to ensure perfect area coverage. Node sensing ranges $\left(R_{s}\right)$ and communication ranges $\left(R_{c}\right)$ are assumed identical and exactly circular, with $R_{c} \geq 2 R_{s}$ to preserve network connectivity [154]. All nodes within sensing range of an event are assumed to simultaneously detect it.

\subsection{General Approach}

When a node detects an event, an Event NOTIFication (ENOTIF) message is broadcast containing the following information where the size of all fields are situational:

Sender ID (globally unique)

Notification ID (unique within the sender)

Sender Coordinates

Forwarder Coordinates (= sender coordinates)

Payload Length

Payload (if any) 
Small packets are more likely to reach their destination [213] so choosing appropriate field sizes can have a significant impact on detection delay; event notifications that fail to reach the sink at the first attempt have to be resent. If a high location accuracy is necessary, a high precision, possibly three dimensional coordinate system will be required; such coordinates require more space in the event notification (message) than a less precise, two dimensional system would demand.

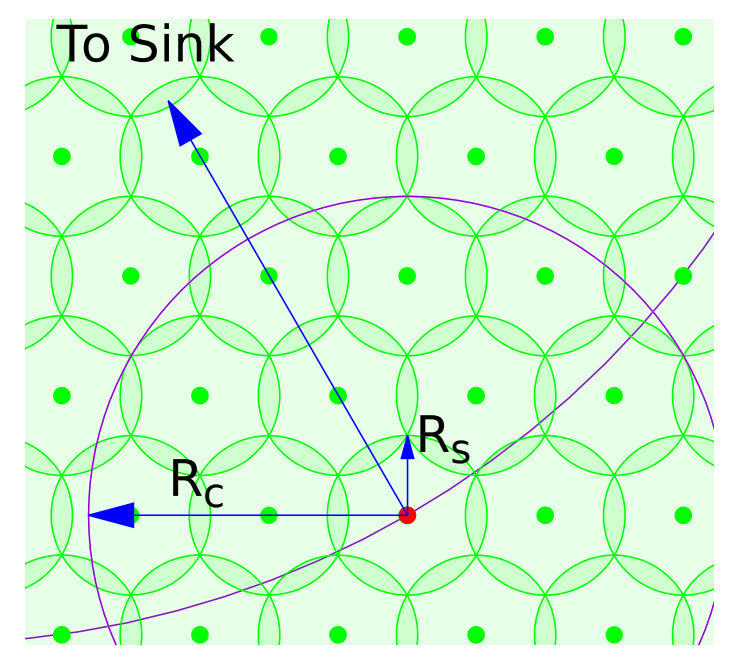

(a) $R_{c}=4 R_{s}$

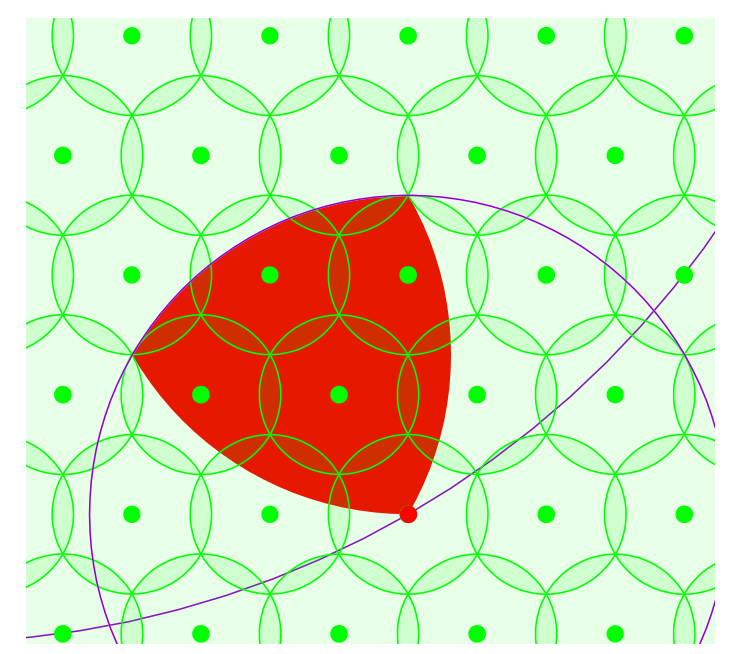

(c) Reuleaux Triangle

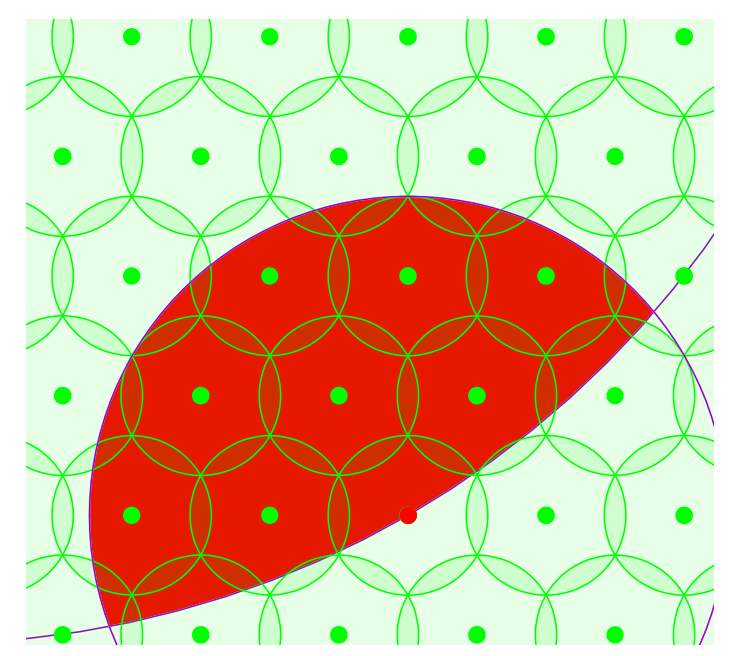

(b) Naive / MFA

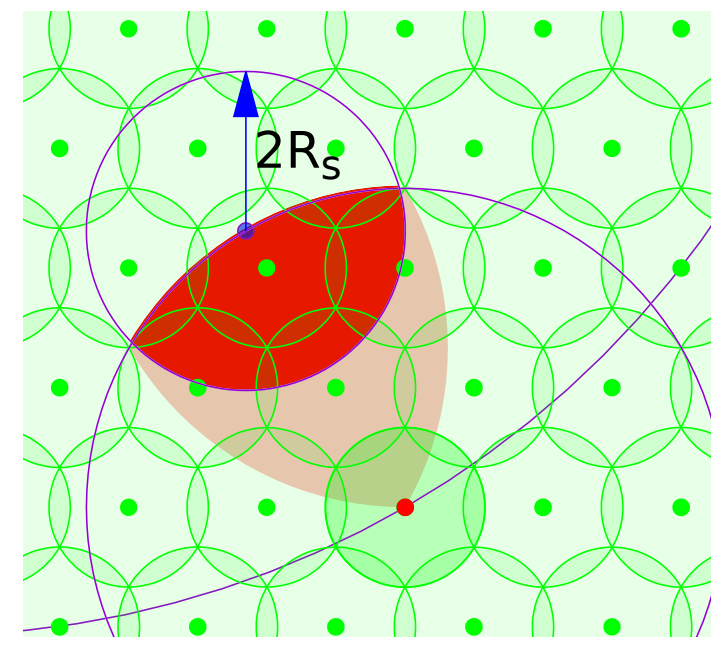

(d) SOFIE's Clipped Reuleaux Triangle

Figure 5.1: Comparison of forwarding regions.

Nodes within $R_{c}$ that successfully receive the message replace the forwarder coordinates with their own and immediately re-broadcast if they are located within the forwarding area highlighted as the darkest area in Figure 5.1d. This forwarding area being the intersection of a Reuleaux Triangle of radius $R_{c}$ oriented to- 
wards the sink with a vertex at the location of the previous forwarding node, and a disk of radius $2 R_{s}$ located at the ideal forwarding position, the point on a direct line from the previous forwarding node to the sink, at a distance of $R_{c}$ away from the forwarding node. Forwarding areas are based on Reuleaux Triangles as a compromise between Maximum Communication Area (MCA) and $60^{\circ}$ Radian Area (DRA), where Maximum Forwarding Area (MFA) is deemed the naive approach [214].

Re-broadcasts occur immediately, increasing the possibility of media contention that will be dealt with by the medium access control (MAC), the forwarding path for any given notification is therefore non-deterministic; Figure 5.2 illustrates the difference between this scheme and greedy forwarding [169] employed by CBF and BOSS.

On receipt of an ENOTIF, the sink, after a short delay $\left(T_{\text {eack }}\right)$ broadcasts an Event $\underline{A C K n o w l e d e g m e n t ~(E A C K) ~ c o n t a i n i n g ~ a l l ~ t h e ~ f i e l d s ~ i n ~ t h e ~ E N O T I F ~ e x c e p t ~}$ the payload and its length, with the forwarder coordinates replaced by those of the sink. $T_{\text {eack }}$ exists to avoid contention between outgoing acknowledgements and incoming notifications. Notifications are implicitly more important than acknowledgments, and are given higher transmission priority.

On receipt of an EACK, nodes re-broadcast after replacing the previous forwarder's coordinates with their own if they are located within a forwarding region similar to the one shown as the darkest area in area in Figure 5.1d, but oriented towards the notification sender, the coordinates of which are implicitly in the acknowledgement as a result of being in the corresponding notification.

To facilitate message suppression (Section 5.2), event de-duplication (Section 5.4), and an optimisation in the provision of event reliability (Section 5.3), SOFIE requires nodes maintain a list of the messages it has previously seen. Practical implementations make this an appropriately sized circular buffer.

\subsection{Message Suppression \& Collision Avoidance}

ENOTIFs and EACKs are suppressed (not re-broadcast) if the potential forwarder node is located outside the forwarding region for the message in question. The geometry of the forwarding region changes with the ratio $R_{c} / R_{s}$. For values of $R_{c}$ at or slightly above $2 R_{s}$, forwarding areas approximate to the Reuleaux Triangle of radius $R_{c}$ with a vertex at the previous forwarding node. As $R_{c}$ increases 


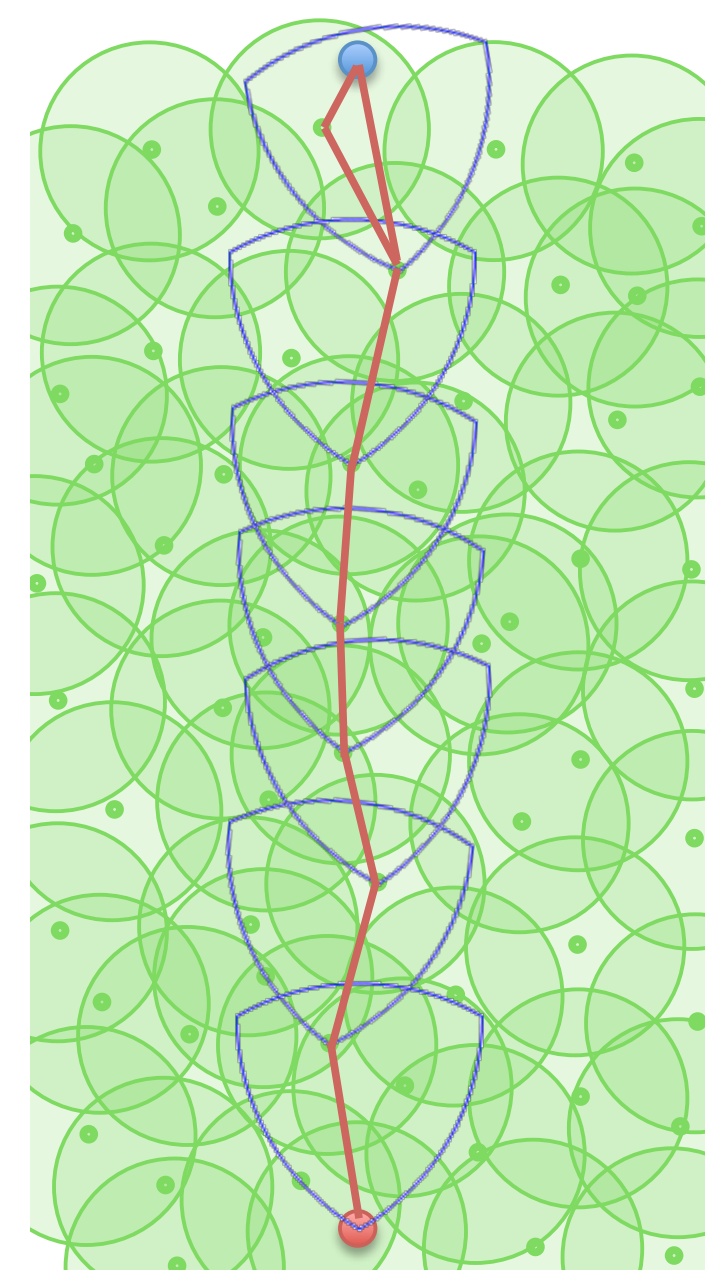

(a)

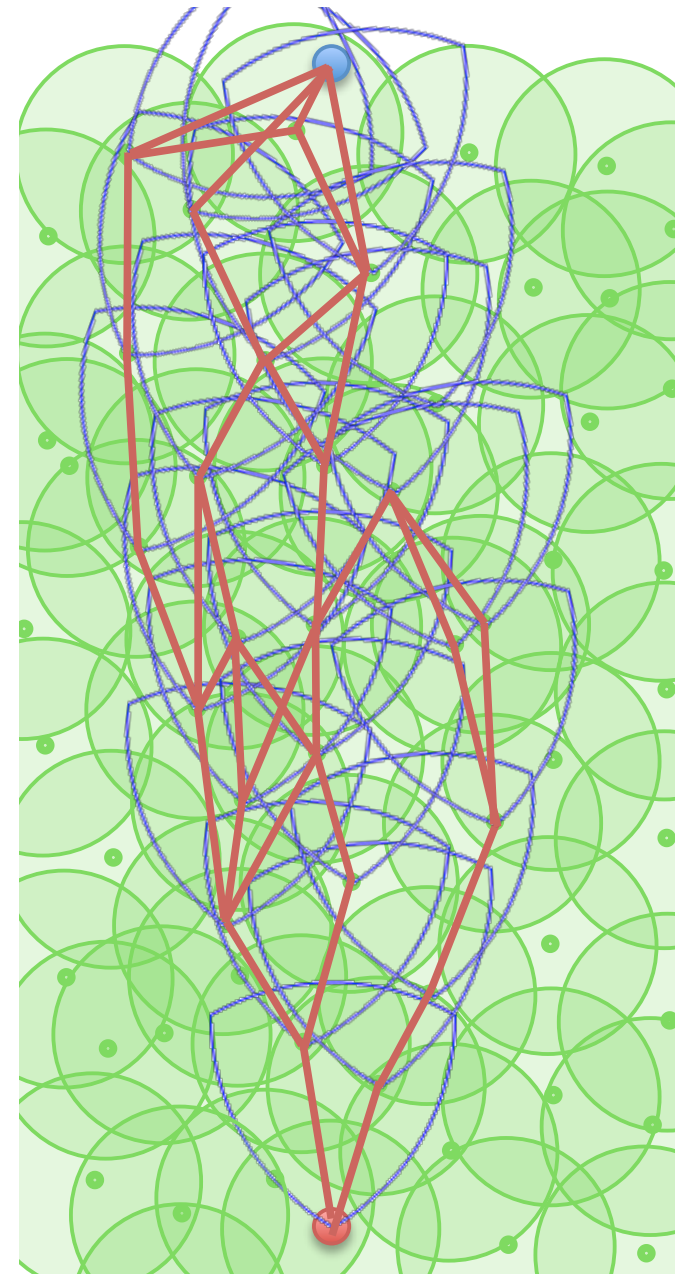

(b)

Figure 5.2: Possible paths for (a) greedy (CBF/BOSS) forwarding; (b) nondeterministic (SOFIE) forwarding. $R_{c}=2 R_{s}$.

for a fixed $R_{s}$, forwarding regions become an increasingly small sub section of the Reuleaux Triangle until $R_{c} / R_{s}>4$ when the forwarding region is simply described by the intersection of the disc of radius $R_{c}$ centered on the previous forwarding node and the disk of radius $2 R_{s}$ centred on the ideal forwarding position, as show in Figure 5.1d.

Other than the delay sinks introduce when broadcasting an EACK, described in Section 5.1, SOFIE does not attempt to avoid collisions, leaving that task to the MAC, e.g. non-beaconed IEEE 802.15.4. When an event occurs, multiple nodes may detect it and simultaneously attempt to broadcast a notification. If the wireless channel is idle, which it is likely to be, given the infrequent nature of rare events, all nodes detecting the event will attempt to send a packet containing an 
ENOTIF. These signals will almost certainly interfere with each other causing the standard MAC (or modifications to it for time-critical events [215]) to make use of its carrier sense multiple access with collision avoidance (CSMA/CA) capabilities to mediate channel access by all competing nodes.

\subsection{Event Reliability}

For minimally effective event detection, only one notification needs to be received by the sink, and all subsequent notifications for the same event are redundant. However, for more sophisticated analysis after the initial notification, such as event location estimation through multilateration, multiple notifications may need to be successfully delivered, though not necessarily all of them. SOFIE assumes individual nodes are unaware of which neighbouring nodes sensed the same event. For the scenario considered here, an event is regarded as having been reliably delivered if at least one notification is received.

After sending a notification upon sensing an event, the notification will be resent if an acknowledgement is not received within a pre-defined short time frame $T_{\text {retry }}$ defined in Equation 5.1:

$$
T_{\text {retry }}=T_{a c k}+\frac{D_{\text {sink }}}{R_{c}} \times T_{\text {hop }}
$$

where $D_{\text {sink }}$ is the distance from the sensing node to the nearest sink and $T_{\text {hop }}$ represents an estimate of the mean time taken for a notification to travel a single hop on its route to the sink.

Setting $T_{\text {hop }}$ too low can result in notification re-sends when an acknowledgement for the original notification has already been broadcast but not yet received. To mitigate this possibility, if a re-sent notification is received by a node that has already forwarded the corresponding acknowledgement, the acknowledgement is re-broadcast and the re-sent notification is suppressed. 


\subsection{Event De-Duplication}

If an event occurs in the intersection of multiple sensing areas, each node generates an ENOTIF, unaware that that its neighbours have done likewise. Whilst the IEEE 802.15.4 CSMA/CA MAC will mediate channel access and each node will get the opportunity to broadcast an ENOTIF, forwarding nodes and ultimately the network sink will receive notification of the same event multiple times. If this is undesirable, an information fusion technique [216] can be used to minimise the number of duplicate event notifications reaching the sink.

SOFIE uses a simple time-based heuristic to determine if a newly received ENOTIF is likely to be a duplicate of one previously forwarded. The configuration parameter $T_{d u p}$ is set to a deployment specific short time and on receipt of an ENOTIF not previously seen, SOFIE iterates over the ENOTIFs seen within $T_{d u p}$. If all of these ENOTIFs originated from a node less than $2 R_{s}$ away from the most recently received ENOTIF, the new ENOTIF is regarded as a duplicate and will not be forwarded. To convince the sending node that the new ENOTIF was forwarded all the way to the network sink, and prevent it resending the ENOTIF, SOFIE generates and broadcasts the appropriate EACK.

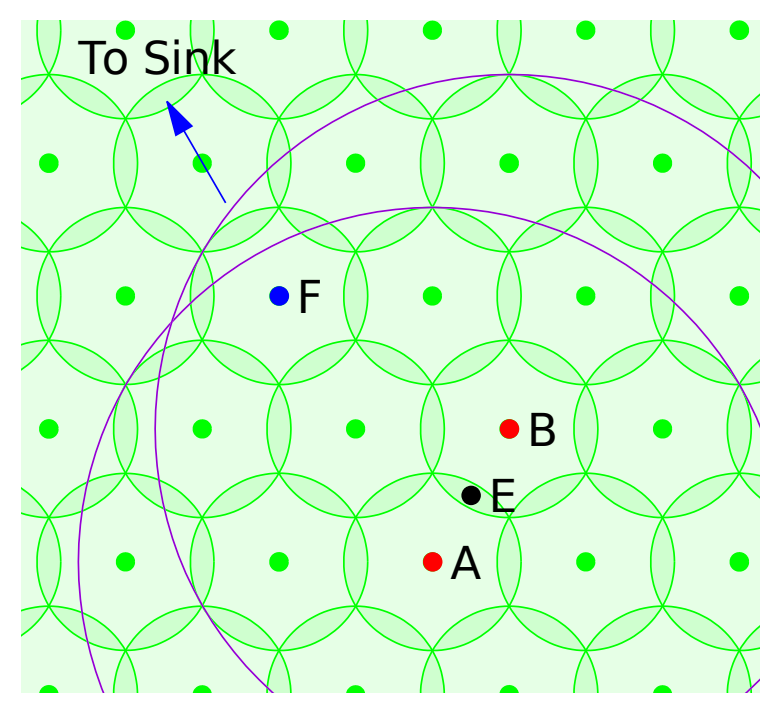

Figure 5.3: SOFIE event de-duplication: A and B detect event at E, F is in forwarding region of both $\mathrm{A} \& \mathrm{~B}$. Green disks represent $R_{s}$, blue disks indicate $R_{c}$ of A \& B. 
For the deployment shown in Figure 5.3, though the order in which devices gain access to the channel is non-deterministic, event de-duplication might proceed as follows:

1. Event occurs at location $E$

2. Nodes $A \& B$ generate ENOTIFs and submit for broadcast

3. $B$ backs-off, allowing $A$ to transmit $E N O T I F_{A}$

4. Node $F$ receives $E N O T I F_{A}$ and submits for re-broadcast

5. $F$ backs-off, allowing $B$ to transmit $E N O T I F_{B}$

6. $F$ receives $E N O T I F_{B}$

7. $F$ transmits $E N O T I F_{A}$

8. $F$ evaluates $E N O T I F_{B}$ against notifications received in the last $T_{d u p}$

9. $F$ identifies $A \& B$ as having intersecting sensing areas and hence $E N O T I F_{B}$ as a containing a duplicate of the event data in $E N O T I F_{A}$

10. $F$ broadcasts $E A C K_{B}$

11. $B$ receives $E A C K_{B}$

12. Network sink eventually receives $E N O T I F_{A}$ and broadcasts $E A C K_{A}$

13. A eventually receives $A C K_{A}$ 


\subsection{SOFIE Pseudo Code}

Pseudo code for SOFIE is shown in Algorithm 6.

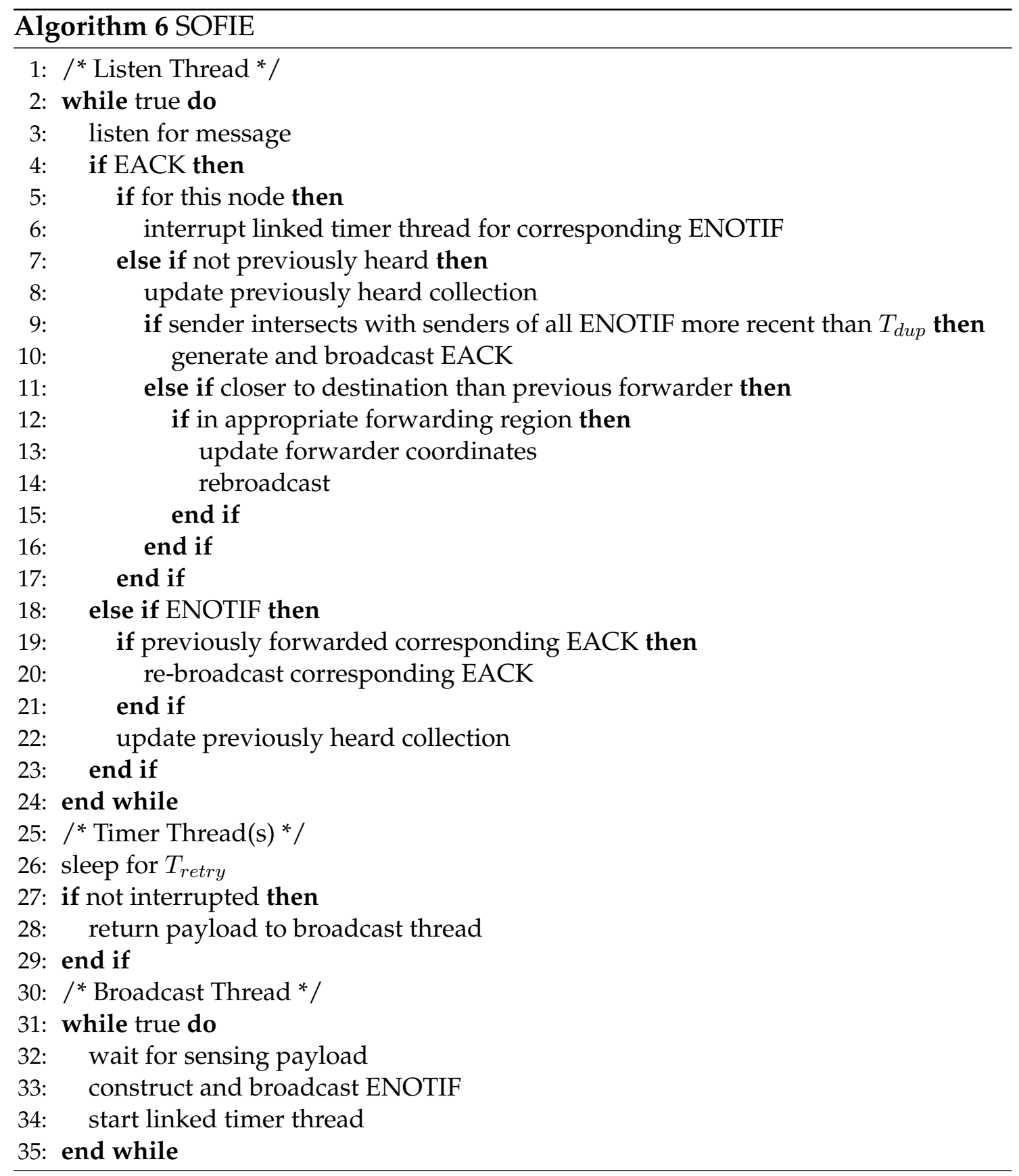




\subsection{De-Duplication Evaluation}

SOFIE is implemented in QualNet 5.2 with all nodes based on Advanticsys CM5000 IEEE 802.15.4 compliant motes configured as non-beaconing full-function devices with CSMA/CA parameters as shown in Table 4.3 from Chapter 4.

Planned deployments of increasing density are generated from the unitary density in Figure 5.4a to twenty times that density. As density increases each node has more extensive intersections with its neighbours sensing areas as indicated by the increasingly dark areas in Figure 5.4b, Figure 5.4c, and Figure 5.4d.

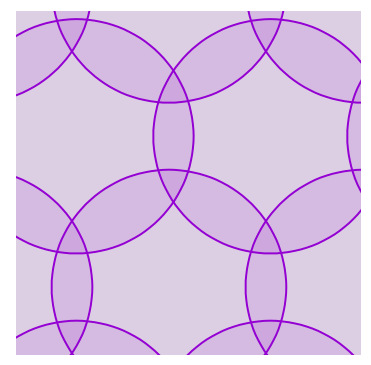

(a)

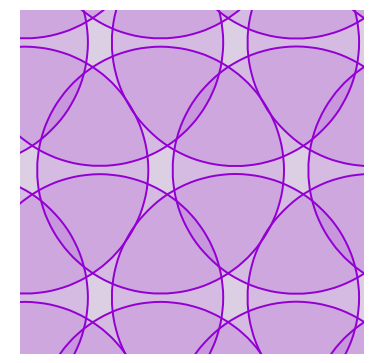

(b)

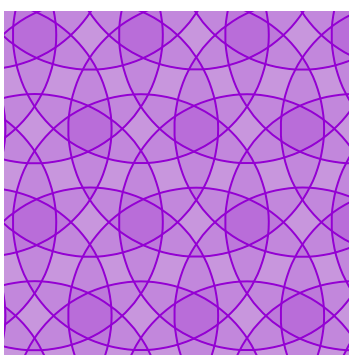

(c)

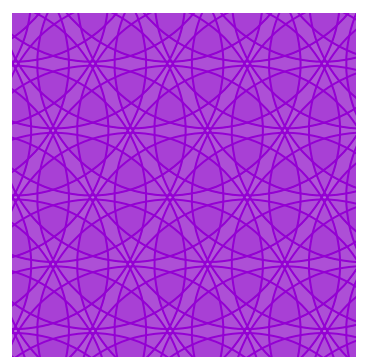

(d)

Figure 5.4: Deployment density: (a) unitary (b) twice as dense (c) three times as dense $(d)$ seven times as dense.

For each deployment density, 500 events are generated 10 simulated seconds apart from each other at random locations within a $200 \mathrm{~m}^{2}$ square sensing area. In all simulations, $T_{a c k}=60 \mathrm{~ms}, T_{\text {hop }}=80 \mathrm{~ms}, T_{\text {dup }}=100 \mathrm{~ms}$ - each simulation being run with 100 distinct random seeds. Each simulation is run with SOFIE deduplication enabled and again with it disabled to determine the efficacy of the mechanism. The following metrics are of interest:

Delay Improvement: Percent decrease (or increase) in event notification delay realised, by enabling de-duplication.

PHY Receive Errors: Percent of signals received with error in the IEEE 802.15.4 PHYsical layer.

Event De-Duplications: Percent of ENOTIFs not forwarded because a duplicate had been detected.

Figure 5.5 charts the mean values across all 100 simulations for each. PHY receive errors increase with density, as do event de-duplications, up to a point. However, even with SOFIE de-duplicating around 30\% of received ENOTIFs, the benefit derived in terms of an decrease in delivery delay is negligible; never rising above 
$0.1 \%$. De-duplications show no further increase beyond the point where the deployment density is such that some proportion of nodes detecting the event have to back-off sufficiently often that their ENOTIFs reach forwarding nodes more than $T_{d u p}$ after the first ENOTIF from at least one neighbouring node.

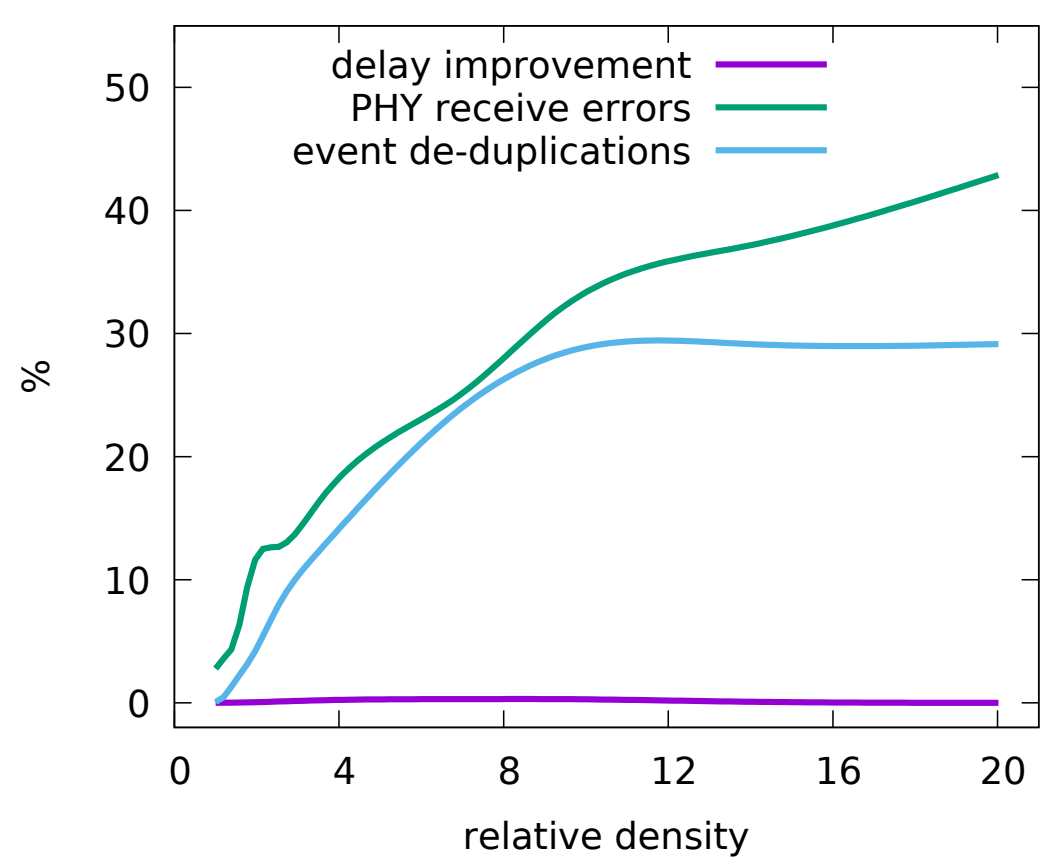

Figure 5.5: Delay improvement, PHY receive errors, \& event de-duplications, $T_{d u p}=100 \mathrm{~ms}$. Spline interpolation with 100 samples.

\subsection{Comparative Evaluation}

CBF and BOSS are also implemented in QualNet 5.2 with all nodes based on Advanticsys CM5000 IEEE 802.15.4 compliant motes configured as non-beaconing full-function devices with CSMA/CA parameters as shown in Table 4.3.

Nodes are deployed in a $400 \mathrm{~m} \times 400 \mathrm{~m}$ perfectly flat sensing area. A sink is placed at one corner of the sensing area. Coordinate fields in all messages are 32 bits, all others are 16 bits with the exception of the payload which varies by evaluation. A total of 109 sensing events occur at pre-determined random locations throughout the sensing area at pre-determined random times over a simulated six hour period. In all cases, $100 \%$ of the events are detected by at least one node and all notifications are successfully received by the network sink. 
Notification delay, the mean elapsed time for the first event notification message to reach the network sink, is the only metric of interest. Hops taken, transmission errors, energy consumed whilst transmitting and receiving, and the proportion of event notifications received at the first attempt, i.e. those received without requiring a re-transmission, are examined to reason about changes in notification delay, but are not of prime interest.

CBF uses an area suppression scheme based on a forwarding area restricted to a Reuleaux Triangle as shown in Figure 5.1c and exposes a single tunable parameter, $T_{c b f}$, representing the maximum time a forwarding node waits before forwarding a received message. For a given forwarding node, CBF waits $R_{c} / D_{\text {prev }} \times T_{c b f}$, where $D_{\text {prev }}$ is the node's euclidean distance from the previous forwarder, before re-broadcasting the message. If whilst waiting to re-broadcast, the node receives a re-broadcast of the same message from a node closer to the sink, the waiting node suppresses the message by not re-broadcasting it.

BOSS nodes also suppress messages based on receiving re-broadcasts from more advantageously placed nodes whilst waiting to forward the same message. The suppression algorithm is based on Discrete Dynamic Forwarding Delay (DDFD), itself based on a weighted banding of the naive forwarding area shown in Figure 5.1b, where the number of bands are configurable, as the Number of Sub Areas (NSA), and the maximum suppression delay, represented here as $T_{\text {boss }}$.

Whilst CBF and SOFIE have a single in-bound message type, BOSS is predicated on research that shows small wireless transmissions are more likely to be error free than large transmissions, and hence uses three types of light-weight coordination messages to avoid re-broadcast of heavy-weight data packets. BOSS's primary motivation is to avoid collisions and consume minimal bandwidth, this being achieved at the expense of extended propagation delays. This clearly contradicts the goal of achieving low latency detection delay of rare event data.

In all evaluations, $T_{a c k}=60 \mathrm{~ms}, T_{\text {hop }}=80 \mathrm{~ms}, T_{c b f}=T_{b o s s}=45 \mathrm{~ms}$, and BOSS $N S A=10$. Each simulation is repeated with 100 distinct random seeds that affect occurrence of errors in the simulated physical layer and one element of DDFD in BOSS.

With $R_{c} / R_{s}=2.2$ for fixed $R_{s}$, hexagon based planned node layouts of increasing density are generated, starting with the ideal placement shown in Figure 5.6a where sensing area overlap is minimised. Similarly, random placements of increasing node density and perfect area coverage are generated, their minimum density necessarily being higher than the planned layouts. Figure 5.6b 
shows a random placement of density 0.0011 nodes $/ \mathrm{m}^{2}$.

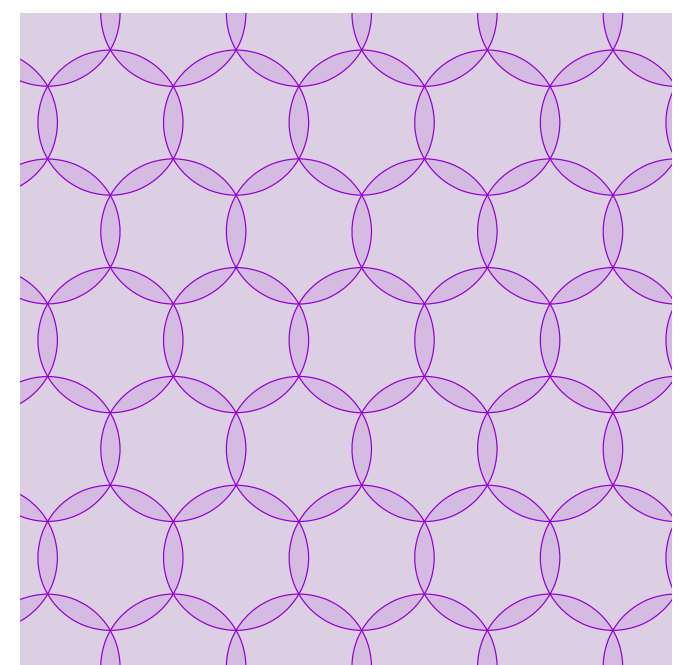

(a) ideal (hexagonal)

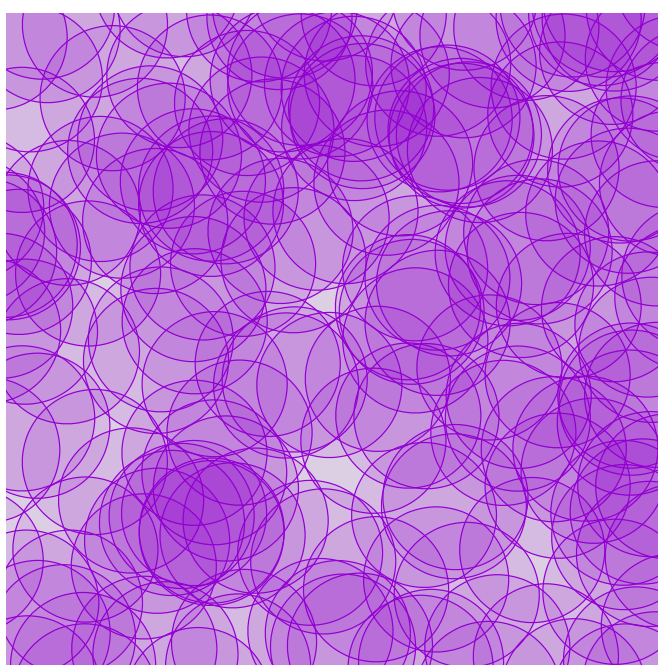

(b) random (Poisson point distribution)

Figure 5.6: Sensing node placements providing 100\% sensing area coverage. $R_{s}$ indicated by circles, overlap is shaded proportionally. $R_{c}$ not shown.

\subsubsection{Delivery Ratio}

CBF and BOSS do not guarantee data delivery even in a network known to be connected. Figure 5.7a and Figure 5.7b show SOFIE achieves perfect event notification delivery for the planned and random placements, described in Section 5.7 and shown in Figure 5.6. To add an equivalent guarantee of event notification to CBF and BOSS, evaluations described in Section 5.7.2 and Section 5.7.3 are undertaken after adding Automatic Repeat Request (ARQ) functionality to the application layer. This ARQ implementation is functionally equivalent to SOFIE's aggressive notification resend algorithm for event reliability described in Section 5.3 . 


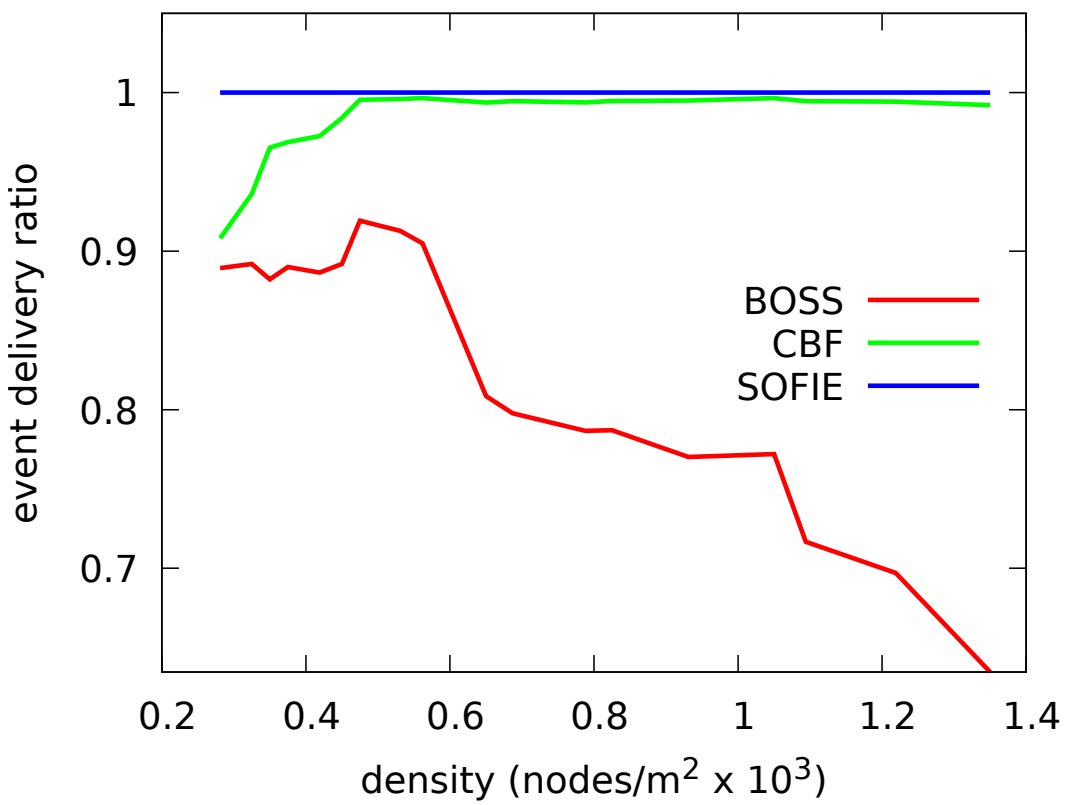

(a) ideal placement

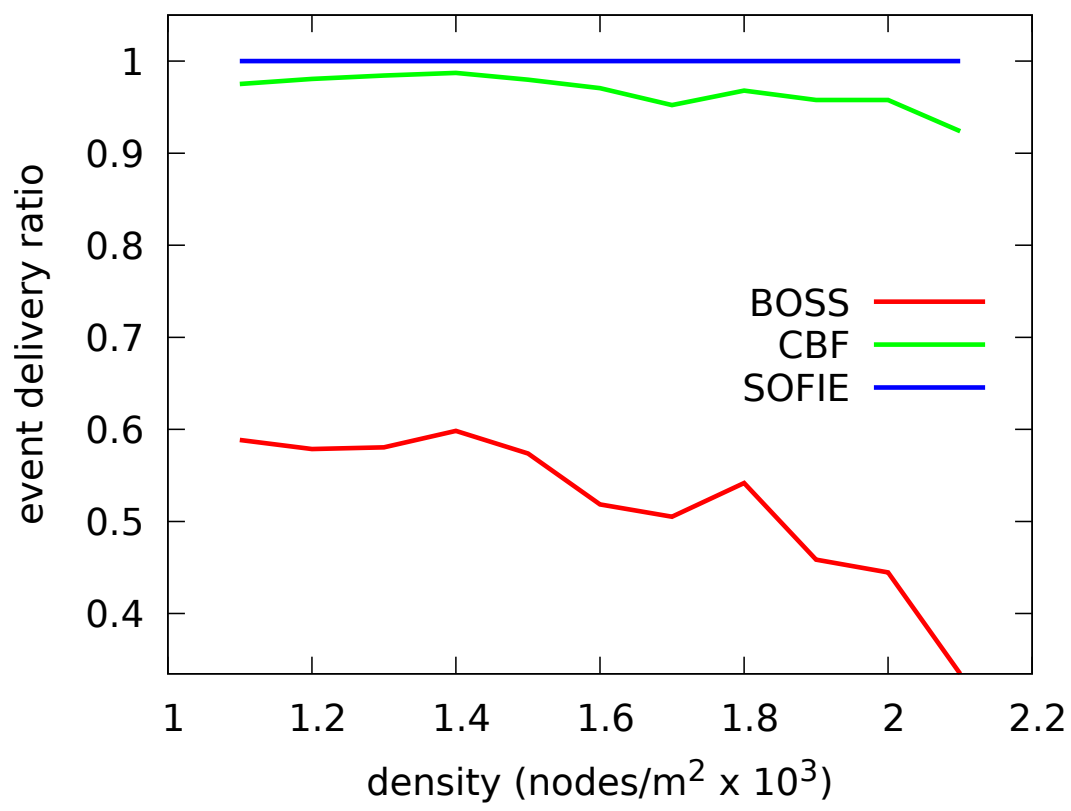

(b) random placement

Figure 5.7: Delivery ratio by node density. 


\subsubsection{Fixed Topology}

Figure 5.8a and Figure 5.8b show SOFIE maintains a lower event notification delay for 64 byte payloads in all circumstances. For planned placements, SOFIE experiences more receive errors than CBF or BOSS as their more cautious forwarding strategies are, at least in part, designed to avoid collisions. Figure 5.9a shows a clear distinction between receive errors, especially at low node densities.

At higher node densities, and particularly in random distributions, the difference in receive errors is less significant, as shown in Figure 5.9b. Energy consumed in transmit and receive states, Figure 5.10a and Figure 5.10b, is higher for CBF and BOSS than for SOFIE. This is a side-effect of the aggressive application layer ARQ modification described in Section 5.7.1 that results in a high incidence of re-sends by originating CBF and BOSS nodes, and the resulting increase in transmitted packets inevitably increasing the energy cost.

Whilst BOSS successfully achieves the lowest error rates, as it was designed to do, it does so at the expense of increased notification delay and by doing so, disqualifies itself as a suitable forwarding protocol for critical events. The following sections will, therefore, compare only SOFIE and CBF.

\subsubsection{Variable Topology}

Extending the operational life of a network deployed to sense critical rare events can be achieved by (a) over populating the sensing area with more nodes than are minimally necessary for perfect coverage and (b) collaboratively duty cycling the nodes so energy utilisation is equalised whilst sensing coverage is preserved [1]. As nodes collaboratively power on and off, the network topology changes making this scenario an ideal candidate for opportunistic forwarding.

Here, an overpopulation of 240 nodes are randomly placed in the sensing area, from which 48 subsets of 140 nodes are pre-selected such that each subset provides perfect area coverage. Individual nodes can be in zero or more subsets. Every 12 seconds a new subset is activated; nodes in the new subset that are not currently energized are powered up, and the 100 nodes not in the new subset are powered down. Note that a given node may be in both the previously active subset and the new one, in which case it remains energized. Nodes also remain energized if they are currently waiting for an acknowledgement of a previously sent event notification; once the acknowledgement is received, the node then powers down unless it is now in the current active subset. Attention is drawn to the rela- 


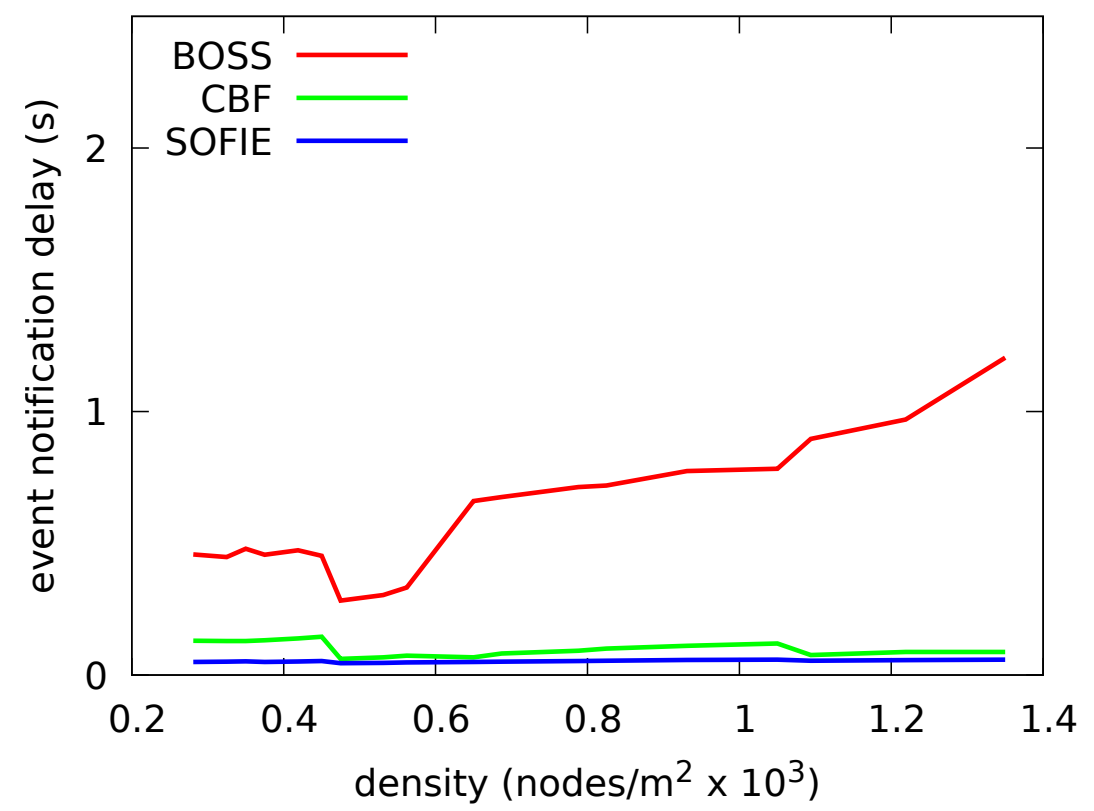

(a) planned, hexagonal placements

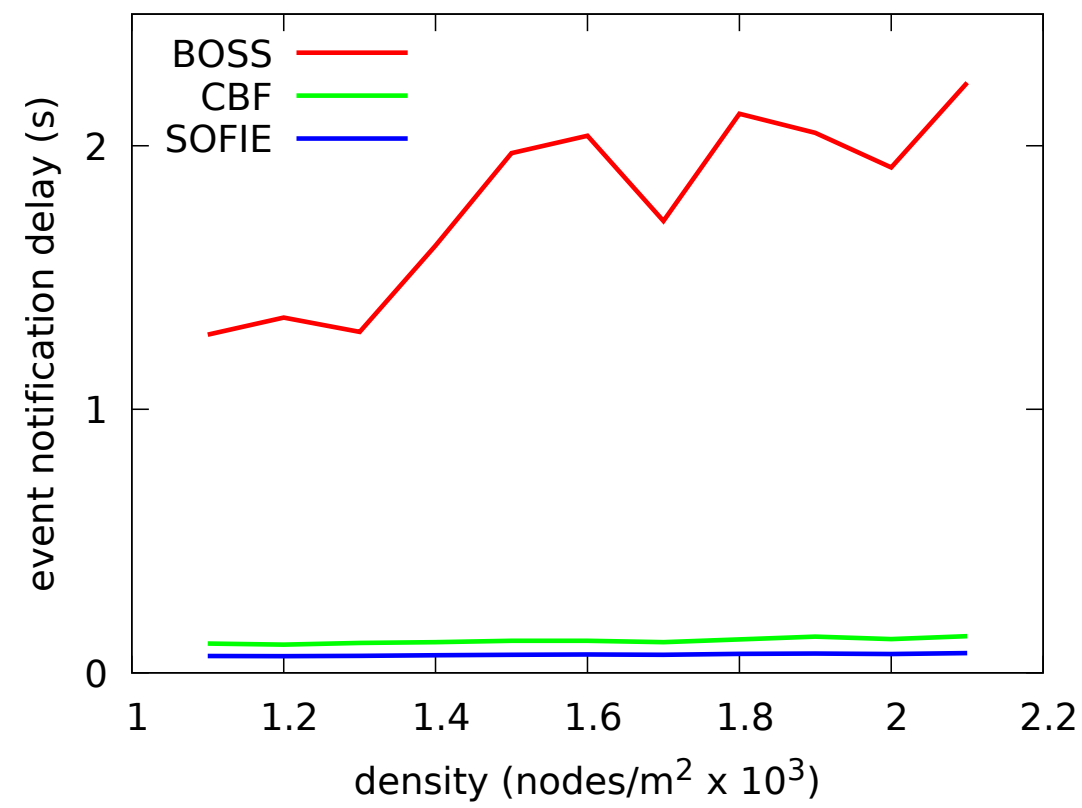

(b) Poisson distributed random placements

Figure 5.8: Notification Delay for 64 byte payloads in placements of increasing node density. 


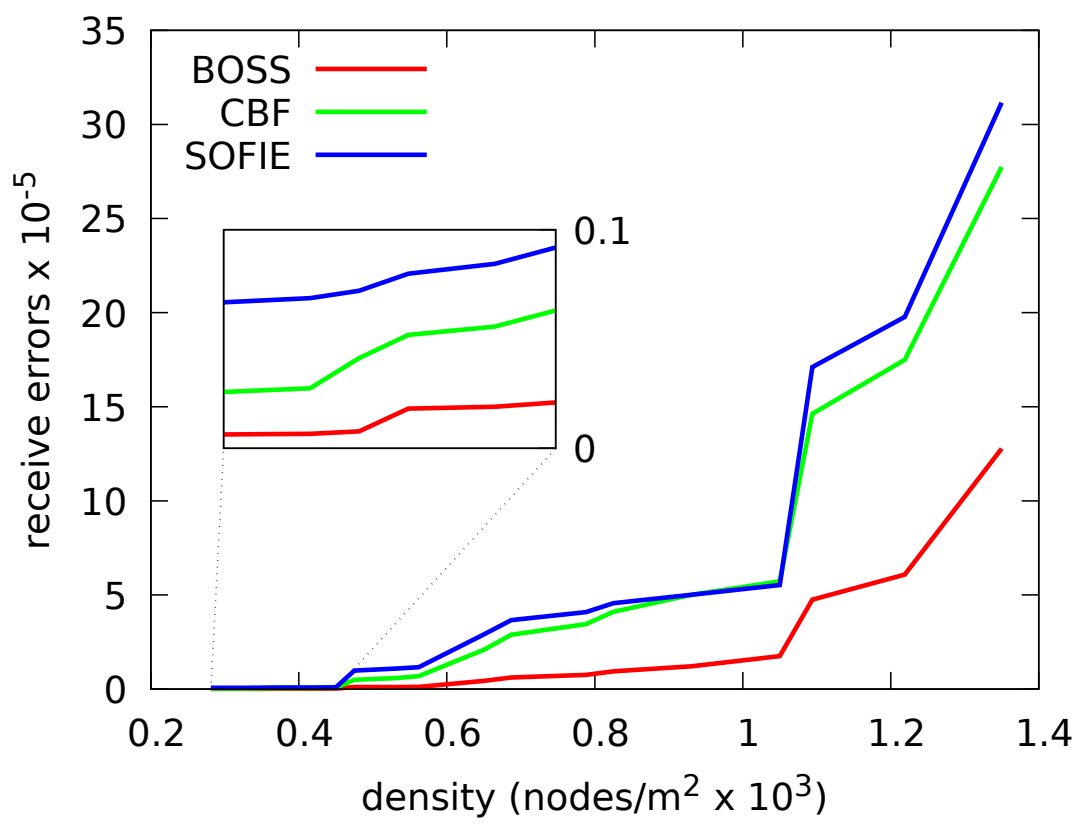

(a) planned, hexagonal placements

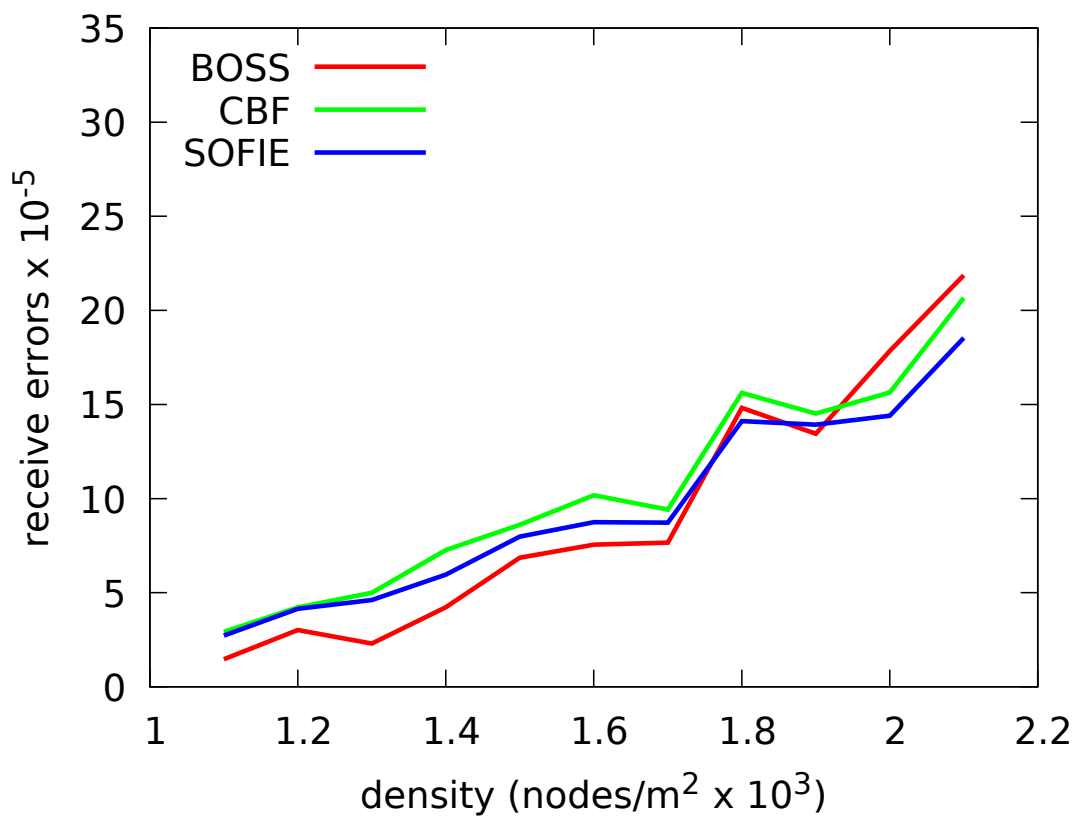

(b) Poisson distributed random placements

Figure 5.9: Receive Errors for 64 byte payloads in placements of increasing node density. 


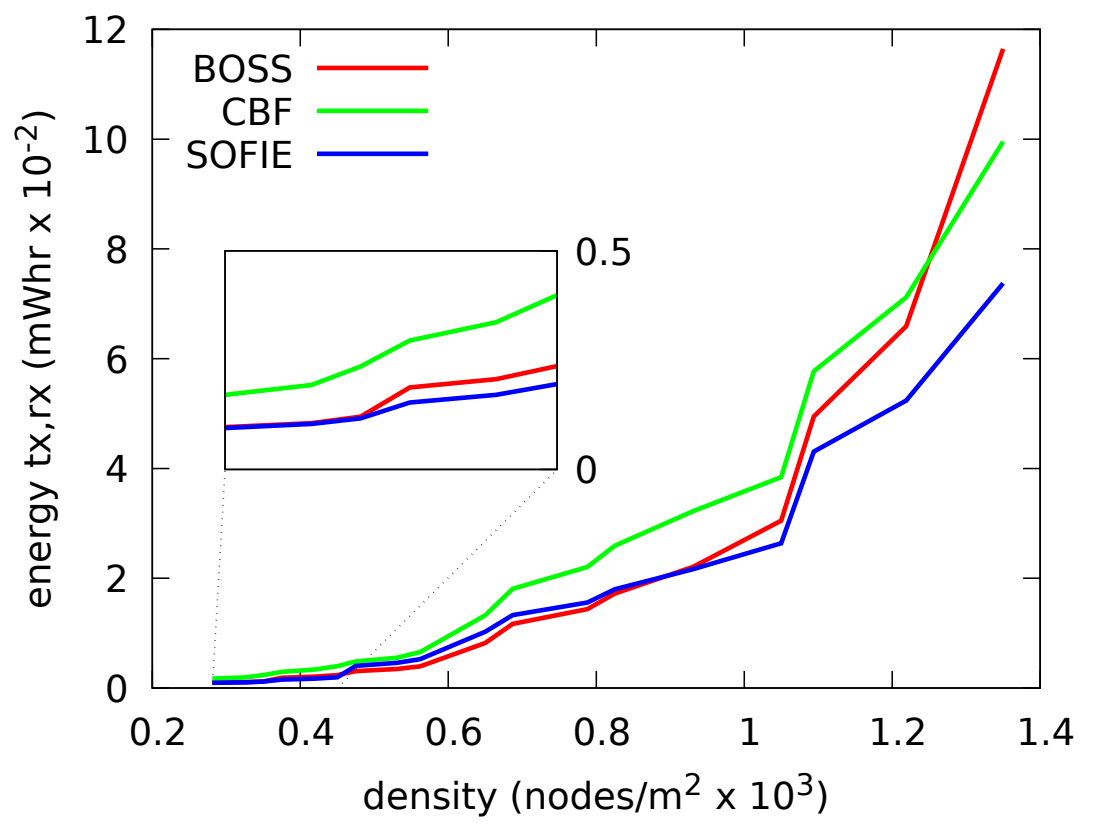

(a) planned, hexagonal placements

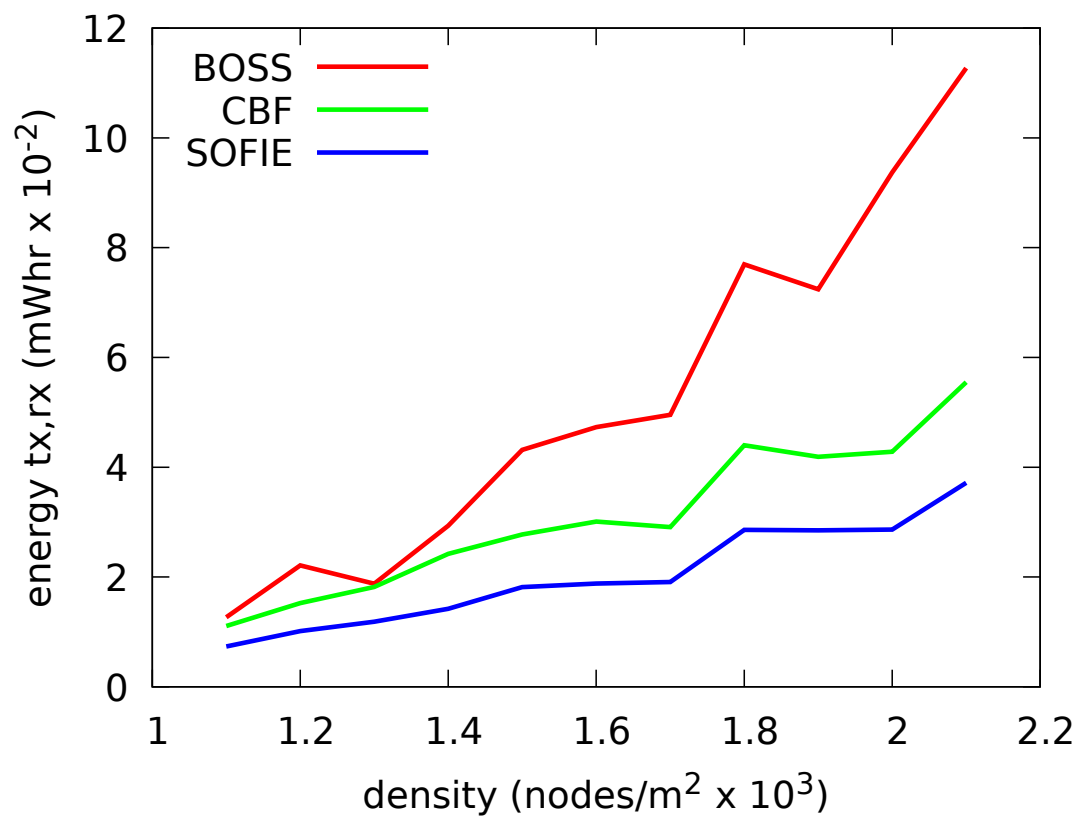

(b) Poisson distributed random placements

Figure 5.10: Energy Consumed in non idle transceiver states for 64 byte payloads in placements of increasing node density. 
tive frequency of duty cycling operations compared to event occurrences; during each 6-hour simulation, nodes duty cycle 1,800 times whereas only 109 events occur.

Evaluations undertaken in Section 5.7.2 used a 64 byte sensing data payload, which is more than half the maximum payload capacity for a single unencrypted IEEE 802.15.4 packet. Packet size is known to affect error rates in wireless networks [213]; in certain sensing scenarios receipt of notification message with a zero length payload may be sufficient to indicate an event has occurred. Here, payloads of 0 bytes and 32 bytes are used.

Figure 5.11a and 5.11b show notification delay for increasing $R_{c} / R_{s}$ for payloads of 0 bytes and 32 bytes respectively. $R_{c} / R_{s}$ was modified by increasing $R_{c}$ for fixed $R_{s}$, the effect of which was to reduce the number of hops for each notification, as shown in Figure 5.12a and 5.12b. SOFIE maintains its notification delay advantage over $\mathrm{CBF}$, the difference being most significant for the most efficient, lower values of $R_{c} / R_{s}$.

\subsubsection{Optimum ratio of $R_{c}$ to $R_{s}$}

In Section 5.7.3, $R_{c}$ was increased while keeping $R_{s}$ constant. On the other hand, when $R_{s}$ is decreased while keeping $R_{c}$ constant, the ratio change is the same but the effect on CBF and SOFIE is noticeably different. In this simulation, $R_{s}$ starts at exactly $\frac{1}{2} R_{c}$ and decreases until it is $\frac{1}{8} R_{c}$ for payload lengths between 0 bytes and 32 bytes. To maintain perfect area coverage, as $R_{s}$ is reduced, node density increases.

Figure 5.13a and Figure 5.13b show that for $R_{c} / R_{s}=2$, SOFIE achieves lower notification delays than CBF, and continues to show this advantage as $R_{s}$ reduces. However, as $R_{c}$ approaches $3 R_{s}$, the advantage is lost. Regardless of the sensing payload size, SOFIE will eventually exhibit a higher notification delay than CBF especially at large $R_{c} / R_{s}$ ratios; this occurs when $R_{c} \approx 6 R_{s}$.

The distance based suppression algorithm in CBF leaves it susceptible to sharp changes in notification delay in planned placement networks as the density oscillates around values that leave the best placed next forwarding node close to the ideal position; the ideal forwarding position being one that allows CBF to select a very short suppression delay leading to minimal notification delay. 


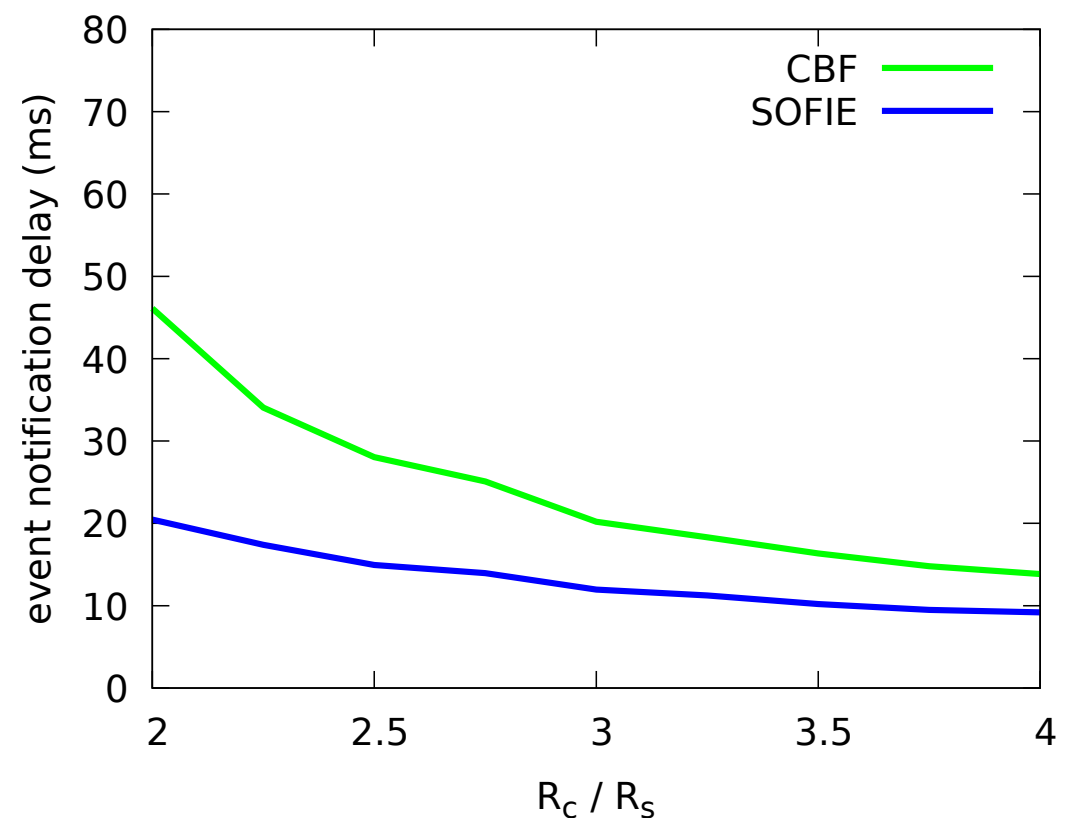

(a) 0 byte payload

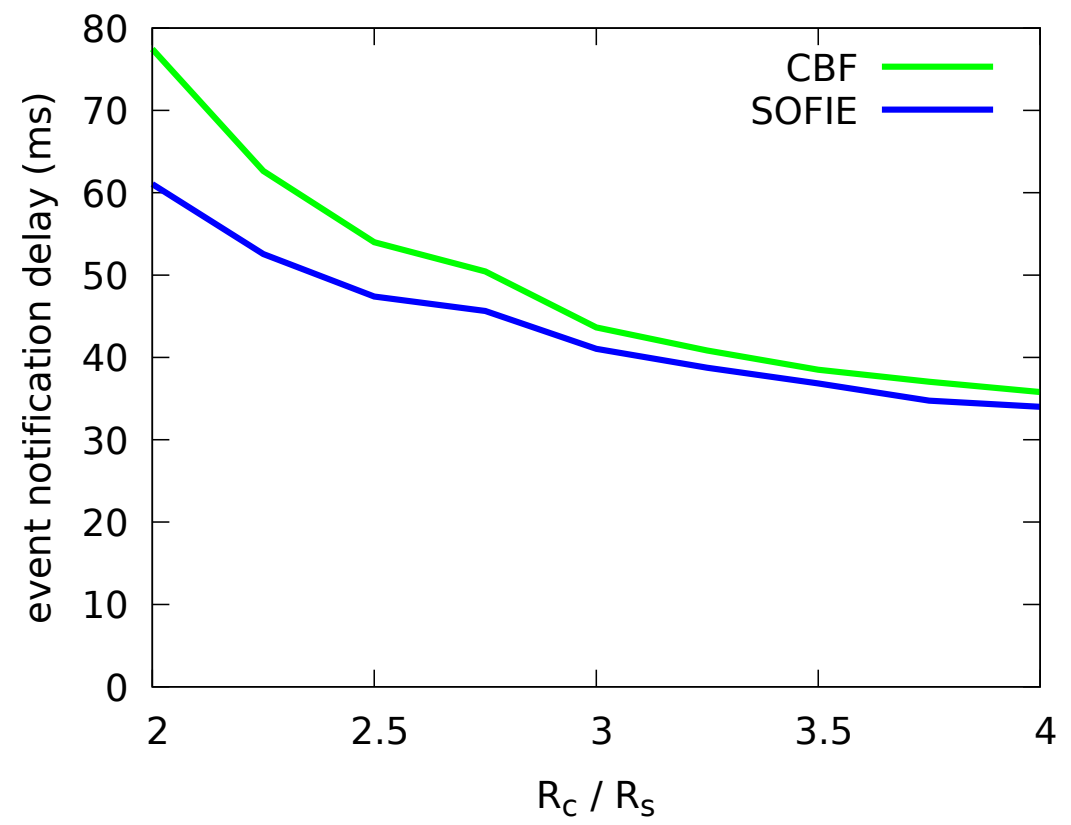

(b) 32 byte payload

Figure 5.11: Notification delay for coverage preserving, sleep-scheduled network with active node density of 0.00088 nodes $/ \mathrm{m}^{2}$ by increasing $R_{c}$ for fixed $R_{s}$. 


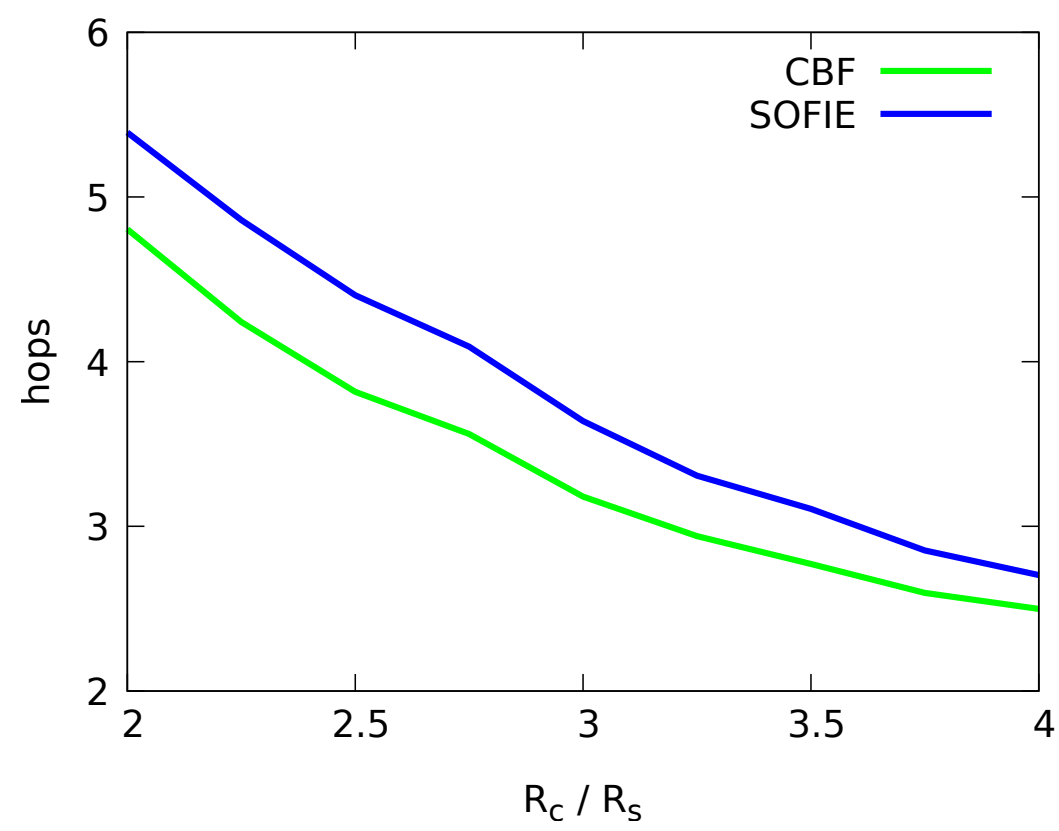

(a) 0 byte payload

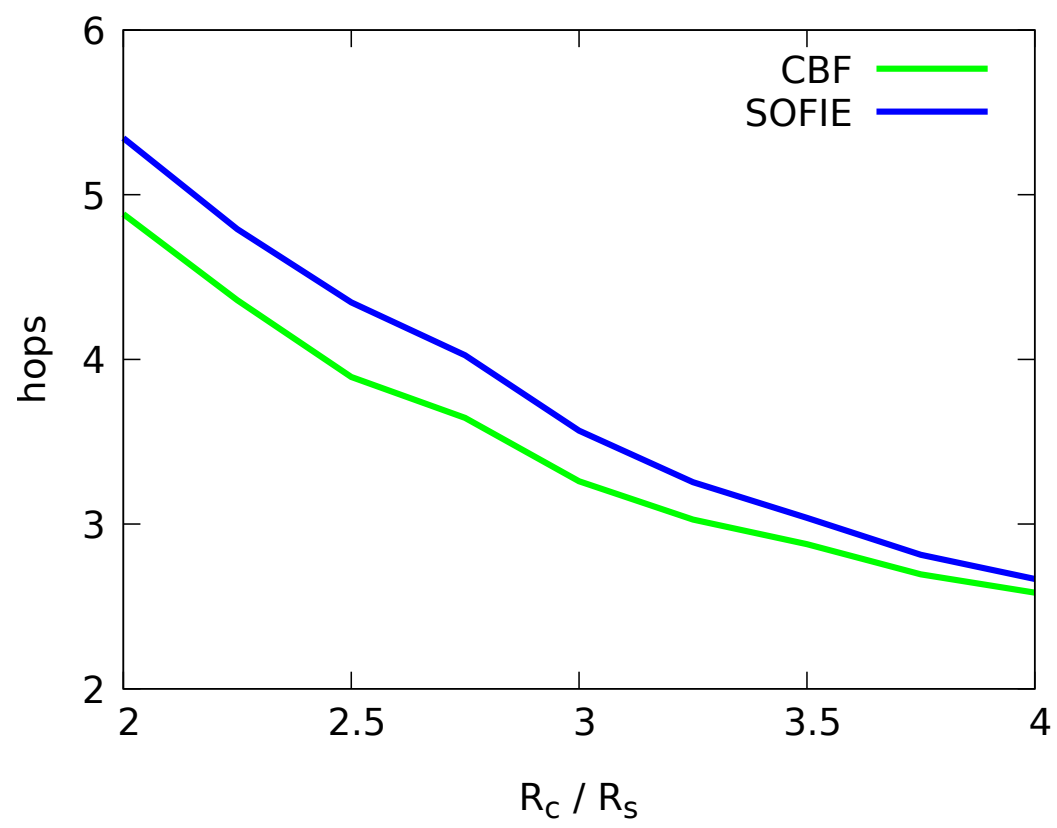

(b) 32 byte payload

Figure 5.12: Hops taken for coverage preserving, sleep-scheduled network with active node density of 0.00088 nodes $/ m^{2}$ by increasing $R_{c}$ for fixed $R_{s}$. 


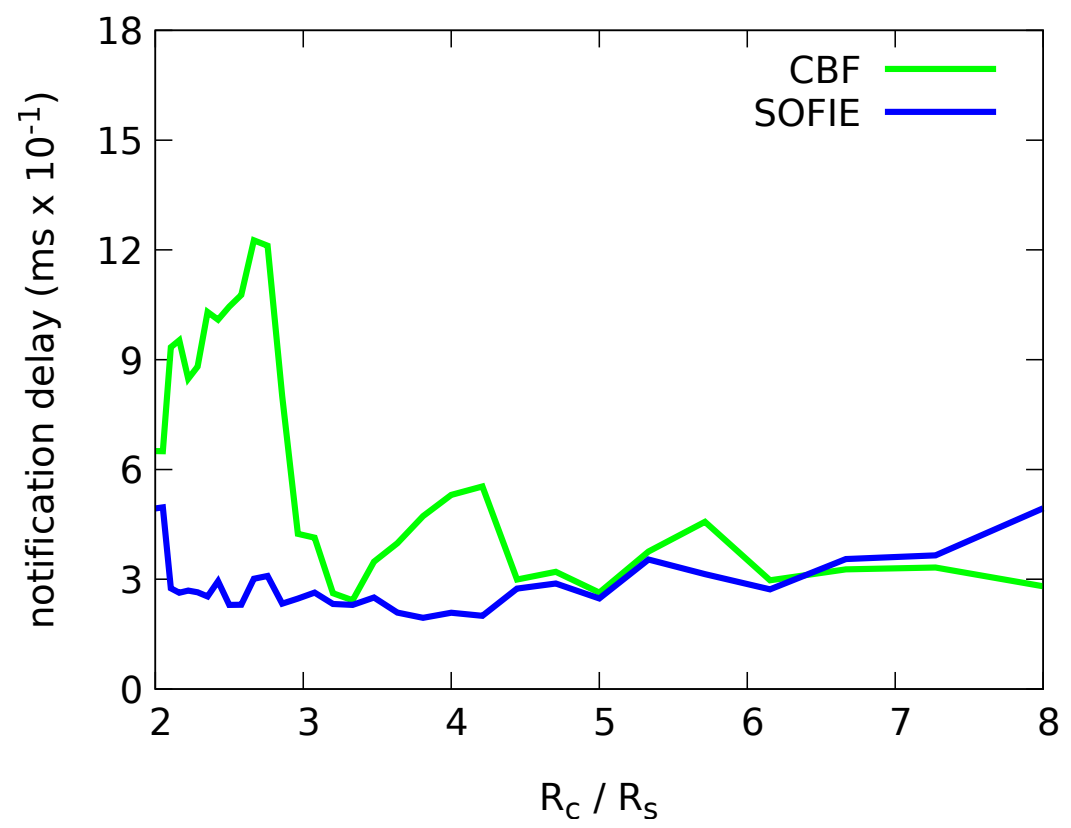

(a) 0 byte payload

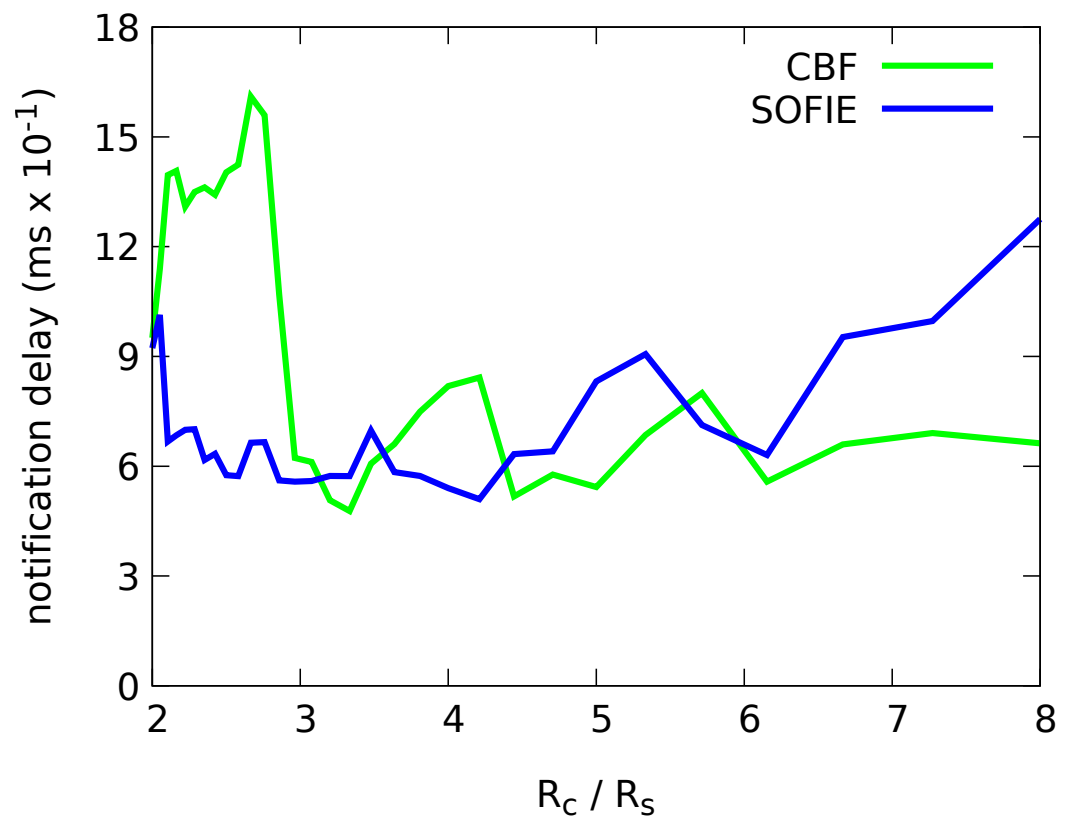

(b) 32 byte payload

Figure 5.13: Notification delay for planned placements with decreasing $R_{s}$ for fixed $R_{c}$. 


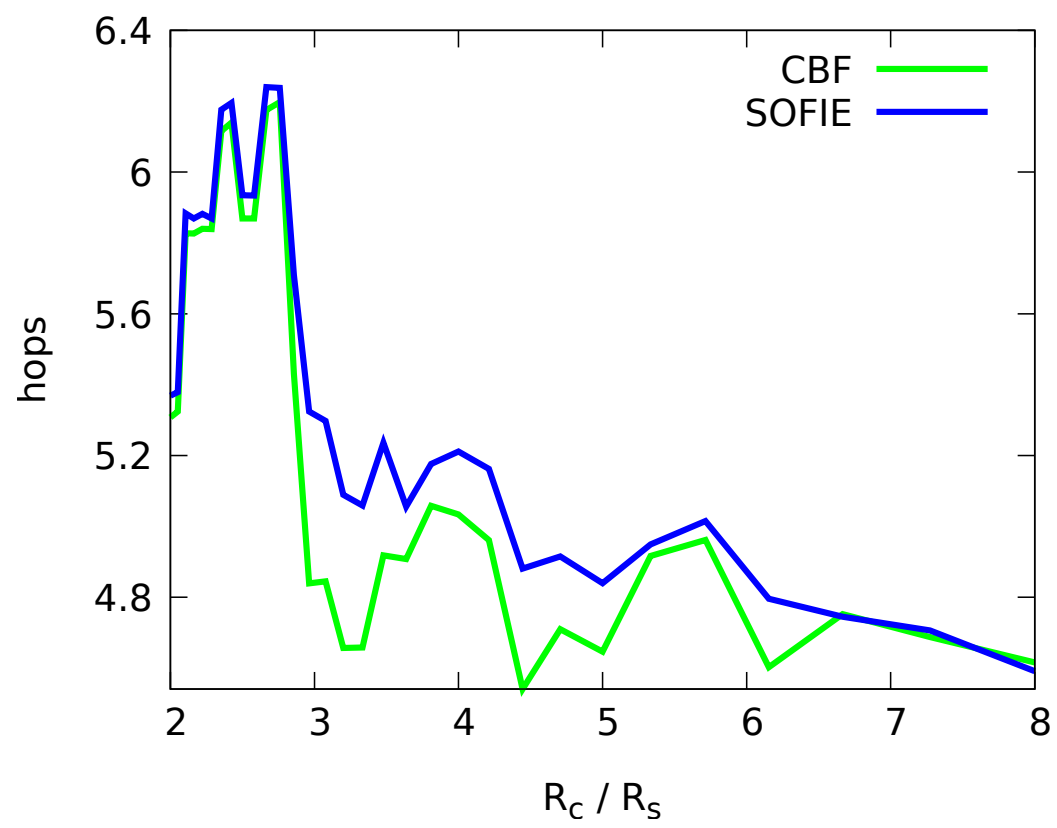

(a) 0 byte payload

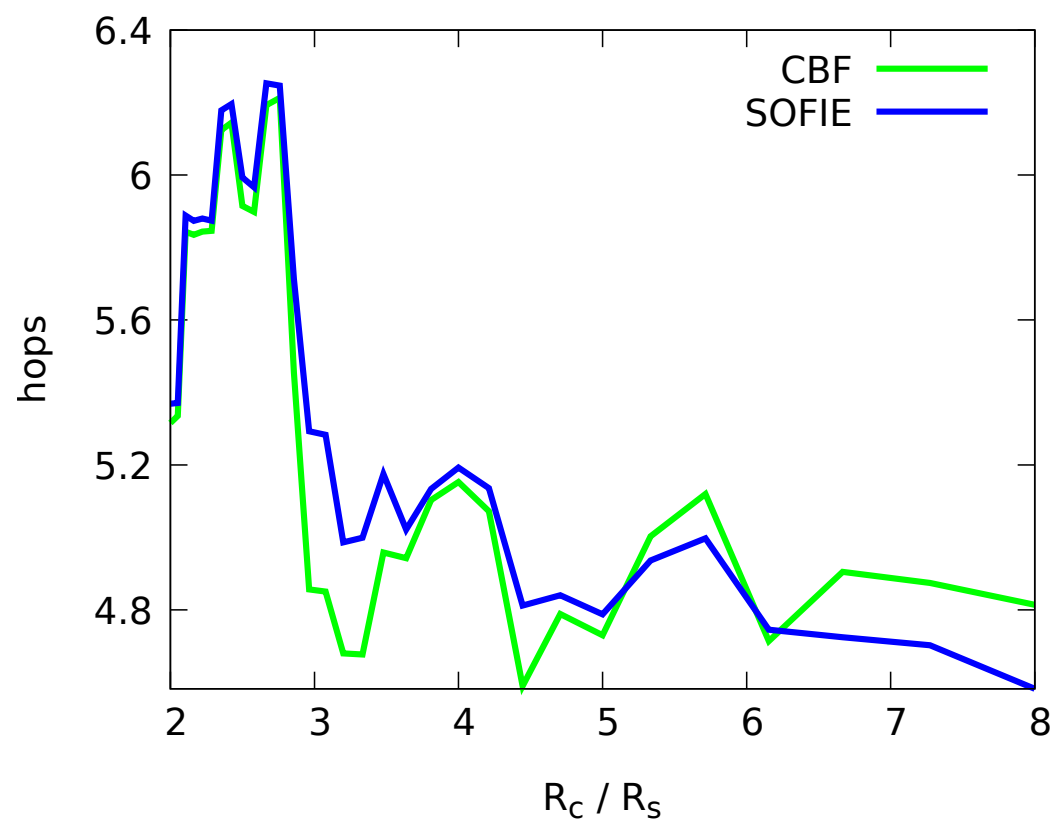

(b) 32 byte payload

Figure 5.14: Hops taken for planned placements with decreasing $R_{s}$ for fixed $R_{c}$. 
SOFIE forwards event notifications on receipt and is consequently less susceptible to changes in node density whilst maintaining a broadly equivalent hop count, an indication that the routes taken by forwarded messages are similar regardless of forwarding algorithm, as show in Fig. 5.14a and Figure 5.14b. However, as density increases, fewer event notifications reach the sink at the first attempt leading to increased re-transmissions and increased notification delay.

\subsection{Summary}

In perfectly covered wireless sensor networks, SOFIE achieves at least a $50 \%$ lower event propagation delay than two general purpose geographic forwarding algorithms when node communication range is between two and three times sensing range, regardless of node density and sensing payload size.

At low node densities, most easily achieved by planned placements, this advantage is gained at the cost of a greater channel contention leading to higher transmission (receive) errors; however, at higher node densities, those typified by random distributions, the transmission error ratio between SOFIE and the best compared algorithm narrows. Further, SOFIE maintains a lower event propagation delay in duty cycling networks where changes in topology caused by nodes entering and exiting sleep state occur frequently when compared to the event occurrence rate.

Differences in energy consumption during transmit and receive operations are observed between the algorithms under test. However, as the target scenario requires all active nodes have their transceivers energized at all times, the cost of idle listening incurred by all the compared algorithms will dwarf that of transmit and receive, rendering any observed differences inconsequential.

As deployments become increasingly dense, information fusion via event deduplication successfully removes an increasing volume of notification messages, but the impact on mean notification delay is minimal suggesting the strategy is of little practical use. 


\section{Chapter 6}

\section{Coverage Preservation with Rapid Forwarding}

This chapter introduces an algorithm for Coverage Preservation with Rapid Forwarding (CPRF) in event sensing WSNs exhibiting perfect area coverage, defined as a network where every point in the sensing field is within sensing range of at least one energised node. CPRF is concerned with three things, viz. coverage maintenance, speed of delivery for event notifications messages, and surviving extended periods of low energy harvesting. Whilst different in design, CPRF embraces the coverage and connectivity aims of the Equitable Sleep Coverage Algorithm for Rare Geospatial Occurrences (ESCARGO) described in Chapter 4, and incorporates the algorithm for swift Opportunistic Forwarding of Infrequent Events (SOFIE) from Chapter 5. The efficacy of the integrated algorithm is assessed through simulation, laboratory tests on commercially available IEEE 802.15.4 WSN devices, and live deployment in sparse, uniformly distributed networks of energy harvesting devices.

Of particular interest is ensuring the WSN node that sleeps least, sleeps for as long as possible. Alternatively, if the devices being used are capable of monitoring their reserves of stored charge, the node that experiences the lowest level of stored charge over time retains as much stored charge as possible. Algorithms that deliver a high least sleep or high least charge allow battery powered WSNs to provide a higher degree of sensing coverage for a longer time. Similarly, in energy harvesting networks, a high least sleep percentage allows the WSN to survive periods where little energy is available for harvesting. The node exhibiting the least sleep or least charge is the one that will exhaust its energy supply 
first. When this node runs out of energy, sensing coverage is lost.

No previous work has focused on the unique situation sensing critical rare events introduces, namely the need to minimize detection delay at all costs, yet collision avoidance algorithms that do not de-activate forwarding nodes promise the least impact on latency; CPRF is based, in part, on this observation.

\subsection{Summary of Related Work}

A distributed algorithm ensuring coverage in energy harvesting WSNs has recently been proposed by Yang and Chin [217]. Their Maximum Energy Protection (MEP) protocol addresses the same Distributed Maximum Lifetime Coverage with Energy Harvesting (DMLC-EH) problem as the algorithm presented here. MEP is aimed at continuous sensing of discrete targets at known locations, while our work is focused on event sensing where the location and timing of the event are not known in advance, and continuous area sensing is required. By Yang and Chin's definition, CPRF is a self-configuration protocol, yet in the scenario targeted here the suggested overhead of sending co-ordination messages is minimal compared to the energy lost through the constant idle listening required for instantaneous propagation of event notification messages.

While similar to this work for seeking to address coverage, the Role Alternating Coverage Preserving Coordinated Sleep Algorithm (RACP) [27] for battery powered WSNs specifically excluded connectivity from consideration. Furthermore, detectable events are assumed to occur in a finite set of known locations, with coverage of only these locations being required; our work demands the entire network sensing area be covered as events can occur anywhere within it. In addition, assessment of RACP's efficacy assumes error free transmission and focuses on dense random node distributions with few similarities to the sparse planned placements featured here. Although shown to extend the lifetime of battery-powered WSNs when compared to random sleep cycling and the coverage algorithm proposed by Tian and Georganas [83], RACP has recently been shown [1] to provide sub-optimal coverage preservation in energy harvesting networks when compared to ESCARGO, the coverage preserving algorithm CPRF takes inspiration from.

CCP [218] is a mature coverage preservation algorithm that in networks not conforming to the $R c \geq 2 R_{s}$ rule for connectivity being implied by coverage 
[154] can be integrated with SPAN [155] to re-instate connectivity guarantees. Whilst CCP's distributed sleep scheduling algorithm is elegant and simple to implement, the message exchange between neighbours it depends on is by its nature unreliable and sleep decisions are occasionally based on stale information relating to neighbouring node's state. As such, CCP cannot guarantee sensing coverage.

Few algorithms address both sensing coverage and explicit maintenance of network topology to facilitate forwarding and/or routing; two that do are A3Cov [41], and 3D-kCov-ComFor [219]. Of these two, only 3D-kCov-ComFor provides a built-in forwarding mechanism.

A3Cov is a topology construction algorithm with a non-localized and inexpensive way of producing a connected backbone. Once a connected topology is established, A3Cov adds extra nodes to provide a partial coverage solution. A3Cov is favourably compared to an area-based collaborative sleeping protocol for WSNs (ACOS) [42], yet it does not attempt to provide perfect area coverage, rendering it unsuitable for critical event sensing. Further, the A3Cov algorithm introduces cases in which a node may choose to sleep despite doing potentially compromising connectivity in a section of the network. A timeout based second opportunity process is required to mitigate this problem but does not remove it entirely, adversely impacting notification delay.

3D-kCov-ComFor combines a duty-cycling coverage algorithm and a composite geographic forwarding scheme suitable for three dimensional space. 3DkCov-ComFor requires significantly denser sensor deployments than many competing coverage preservation algorithms, but provides strong coverage guarantees. This ability to maintain sensing coverage comes at the expense of being unable to reduce the number of active nodes to the levels acheived by its competitors. In energy harvesting networks this may prove problematic as the more nodes that can sleep simultaneously, the more likely the network is to survive extended periods of low harvestable energy. This inability to deal with troughs in available energy leads to degradations in detection probability when coverage is lost, and in detection delay when forwarding routes are compromised. Further, 3D-kCov-ComFor restricts its forwarding region to such an extent that in all practical deployments detection delay is significantly degraded compared to algorithms that makes full use of the maximum available transceiver transmit range. 


\subsection{Algorithms Chosen for Comparison}

Only the recently proposed $3 \mathrm{D}-\mathrm{kCov}-\mathrm{ComFor}$ is a true competitor to CPRF, as such it will naturally be used in a comparative assessment. However, as the majority of the literature considers coverage and forwarding to be separate concerns, it is perhaps informative to combine best-of-breed algorithms from both areas and include them in the assessment. To that end, the well-established protocols CCP [218] and CBF [179] will be integrated to provide a benchmark against which 3D$\mathrm{kCov}$-ComFor and CPRF can be evaluated. For the purposes of this investigation, this composite algorithm will be referred to as CCP/CBF.

\subsection{Combined Algorithm}

We assume a two dimensional distribution of location aware wireless sensor nodes, each of which knows the location of all network sinks. Sufficient nodes are placed within the sensing field to ensure total area coverage. Node sensing ranges $\left(R_{s}\right)$ and communication ranges $\left(R_{c}\right)$ are assumed identical and exactly circular, with $R_{c}$ at least $2 R_{s}$ to preserve network connectivity [154]. Non uniform sensing ranges [145] and arbitrary polygonal sensing areas [207] have been studied and CPRF could be implemented for either case without compromising its efficacy. All active (non sleeping) nodes within sensing range of an event are assumed to simultaneously detect it.

To ensure event detection (i.e. guarantee sensing coverage is preserved at all times), and guarantee event detection messages are not only delivered but delivered with minimal delay, CPRF nodes must observe the following rules: (1) Only sleep when safe to do so. Assumptions must not be made about the state of neighbouring nodes. A neighbouring node cannot be assumed to remain awake based on the last message received from it that indicated it was awake. (2) Ensure all messages are delivered. Specifically, take no duty-cycling decisions unless all sent messages have been acknowledged. With these rules in mind, the remainder of this section describes the various aspects of the CPRF algorithm. 


\subsubsection{Rapid Forwarding}

CPRF derives its rapid forwarding capabilities from SOFIE [4], and algorithm containing mechanisms to ensure event notification messages are reliably delivered whilst suppressing duplicates. Collision avoidance is delegated to a carrier sense multiple access with collision avoidance (CSMA/CA) MAC protocol such as that defined by IEEE 802.15.4.

When a node detects an event, an Event NOTIFication (ENOTIF) message is generated. The network sink responds with an Event ACKnowledegment (EACK) on receipt of an ENOTIF. To avoid shutting nodes down before delivery of event sensing messages have been confirmed, CPRF needs to be aware of when an ENOTIF has been sent but the corresponding EACK has yet to be received.

\subsubsection{Sleep Eligibility}

When a node goes to sleep it takes no part in the sensing activities or communication links of the WSN until it wakes up again. To maintain sensing coverage throughout the network, the entire sensing area a sleeping node is responsible for must be covered by one or more neighbouring nodes. Only when a node is confident its sensing area is covered by a set of awake neighbours does it become sleep eligible.

Wang et al. [154] performed geometric analysis of the relationship between connectivity and coverage in networks made up of nodes with uniform sensing and transmission ranges; they state and prove (we use the term neighbour where they use sensor) that:

Theorem $2 A$ convex region $A$ is $k$-covered by a set of neighbours if (1) there exist in $A$ intersection points between neighbours or between neighbours and A's boundary; (2) all intersection points between neighbours are at least $k$-covered; and (3) all intersection points between any neighbour and $A^{\prime}$ s boundary are at least $k$-covered.

This is the intersections $k$-coverage eligibility rule, and CPRF requires nodes to guarantee 1-coverage before entering a sleep state. 


\subsubsection{Sponsor Group Selection}

This thesis principally considers planned node placements, the optimal configuration being based on a repeating and overlapping pattern of three nodes. The three nodes' sensing area perimeters, being of equal length, intersect the vertices of an equilateral triangle of side $2 \sqrt{3} R_{s}$ where $R_{s}$ is the node sensing range, and intersect with each other at the midpoint of the triangle's sides. This placement being identical to one where each node is separated by $\sqrt{3} R_{s}$ from each neighbour [208].

When a third node is added at the intersection of the three existing nodes sensing ranges, the new node is 1-coverage eligible with a single sponsor group made up of the original three nodes. Repeating this pattern using existing nodes as members of new groups leads to a uniform coverage where each node at least 1-coverage eligible.

A sponsor group is a non-redundant subset of a node's neighbours that collectively cover the sensing area of the given node. In the optimal (minimum deployment density) configuration, nodes located at least $R_{s}$ from the sensing area boundary will have five sponsor groups, illustrated in Figure 6.1. Nodes located close to the sensing area boundary have no less than two sponsor groups. Co-located nodes implicitly form one-member sponsor groups for each other.

To ensure successful operation on modest capability devices, CPRF restricts sponsor group size and the number of sponsor groups each nodes is required to maintain with the configurable parameters $S G_{\text {size }}$ and $S G_{\max }$ respectivley.
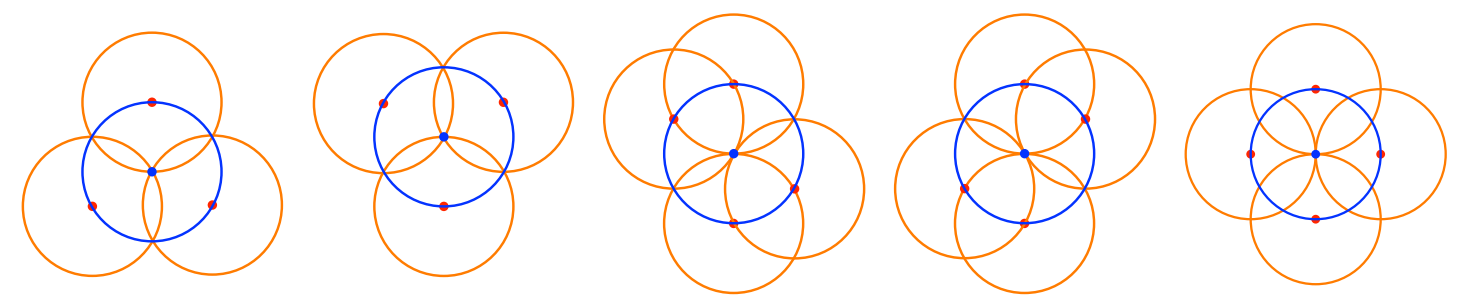

Figure 6.1: Unique, non-redundant sponsor groups for the central node, highlighted darkest. Other combinations of neighbours exist, but all are super-sets of one or more of those shown. Dots represent node locations, circles their sensing areas of radius $R_{s}$. 


\subsubsection{Neighbour Selection}

In sparse deployments each sensing node will have a small number of neighbours, with the neighbours of $A$ being all nodes having Euclidean distance of no more than $2 R_{s}$ from $A$. However, in dense deployments, modest capability devices may have insufficient RAM to store details of all their neighbours. Additionally, maintaining a large neighbour list increases the computation time required to generate all possible sponsor groups, cf. Section 6.3.3. CPRF mitigates these issues by restricting the number of neighbours each nodes is required to deal with to a configurable parameter, $N_{\max }$.

Simply restricting the number of neighbours can lead to an imbalance in the distribution of those neighbours around the node making the restriction. To counter this possibility, CPRF employs the following strategy: First, the sensing disk of node $A$ is repeatedly divided into $120^{\circ}$ segments until the set of three with the most even distribution of neighbour candidates is found. Second, neighbour candidates within the selected segments are ordered by how close the are to being $\frac{2}{3} R_{s}$ from $A$. Third, the next as-yet unselected best positioned node in each segment is chosen in turn until $N_{\max }$ neighbours have been selected.

\subsubsection{Metric Equality}

If coverage is to be preserved across a sleep scheduling WSN, a degree of fairness must be enforced to prevent advantageously positioned nodes from sleeping more often than their neighbours. However, CPRF's approach to sleep scheduling is not simply a fairness algorithm, the aim is to make sure the node that sleeps the least sleeps as much as possible, even if one or more of its neighbours have the opportunity to sleep significantly more often. Over time this tends to have the effect of equalising sleep times across the whole network.

CPRF nodes exchange a metric, the value of which they use to make sleep scheduling decisions, most notably the decision of whether or not to take responsibility for a neighbour's sensing area when asked to do so. For devices that can easily determine their reserves of stored energy this metric can represent the remaining stored charge; the simulations in Section 5.6 take this approach. The commercially available devices used for the live deployment described in Section 4.2.6 do not have the ability to determine their stored charge so we use the percentage of time they have been asleep as the exchanged metric. 
When asked to take responsibility for (sponsor) a neighbour's sensing area, a node will refuse to do so if the requester has a higher metric, i.e. it has more remaining charge or has slept for longer. However, if the sensing node receiving the request is already sponsoring one or more other neighbours, the request will be accepted as to refuse it gains nothing locally (the potential sponsor has to stay awake anyway) and adversely affects the requester by making it stay awake. Naturally, if the requesting node has a lower metric, the receiving node will always accept the sponsorship request.

\subsubsection{Sleep Scheduling}

Nodes are in one of four energised states: SPONSORED where sensing responsibility is delegated to at least one other node, ACTIVE where they are not sleep eligible and may be taking responsibility for the sensing area of one or more neighbouring nodes, SEEKING where they are sleep eligible and attempting to find neighbouring nodes willing to sponsor them, and DEFERRED where they would have transitioned to SPONSORED but are waiting for one or more EACKs. Figure 6.2 shows the algorithm's state transition diagram.

When nodes have sufficient energy to power on they enter ACTIVE state, broadcasting periodic STATus (STAT) messages containing their location, metric, how many neighbours they are current sponsoring and the longest time they would sponsor a node that requested sponsorship but had a lower metric (cf. Section 6.3.5). Nodes listen for STAT messages and determine who their sensing neighbours are. Two nodes are deemed to be sensing neighbours if separated by a Euclidean distance less than or equal to twice their common sensing range, $R_{s}$. Information in STATs received from neighbours is recorded and used to determine sleep eligibility.

A node is eligible to leave ACTIVE state if and only if it determines its sensing area is fully contained within the union set of some combination of its ACTIVE or SEEKING neighbours' sensing areas. Combinations of neighbouring nodes that could sponsor a given node are known as sponsor groups (cf. Section 6.3.2 and Section 6.3.3). Sponsor group membership is determined by geometric calculations taking into consideration the boundary of the sensing area. Nodes adjust their sponsor group lists each time a STAT from a previously unknown sensing neighbour is received. Pseudocode for ACTIVE state is shown in Algorithm 7. 


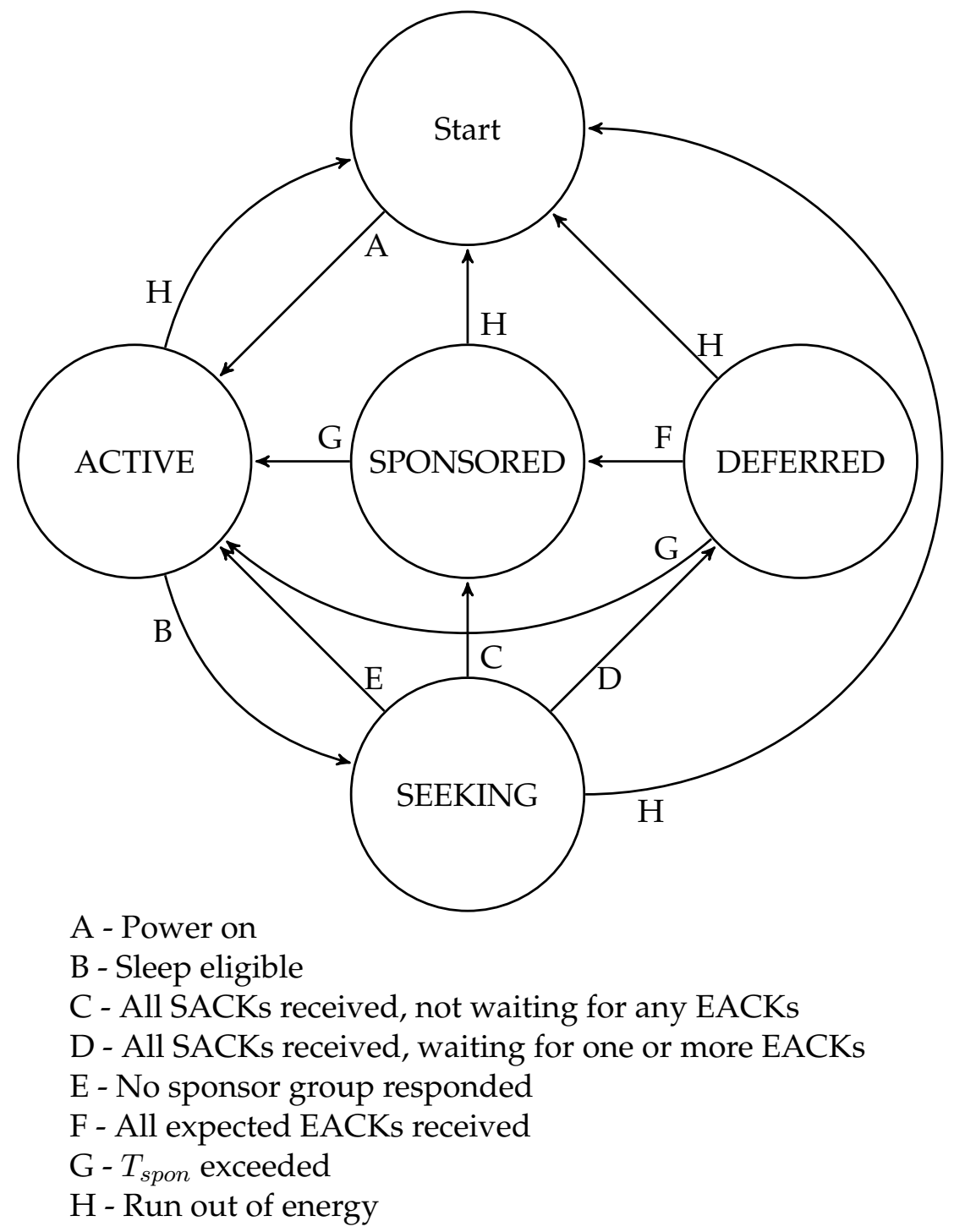

Figure 6.2: CPRF State Transitions

Nodes that deem themselves sleep eligible enter SEEKING state and cycle through the sponsor groups they believe are likely to sponsor them, starting with the one they deem most likely (cf. Section 6.3.2). For each sponsor group selected, a Sponsor REQuest (SREQ) message is broadcast containing identifiers of each neighbour in the sponsor group. Neighbours in receipt of an SREQ in which they are on the list of required sponsors, add the requesting node to their sponsored list and return a Sponsorship ACKnowledgement (SACK) message. Nodes that have agreed to be a sponsor continue to seek their own sponsors until they become aware (through receipt of additional messages) that they are no longer sleep eligible. 


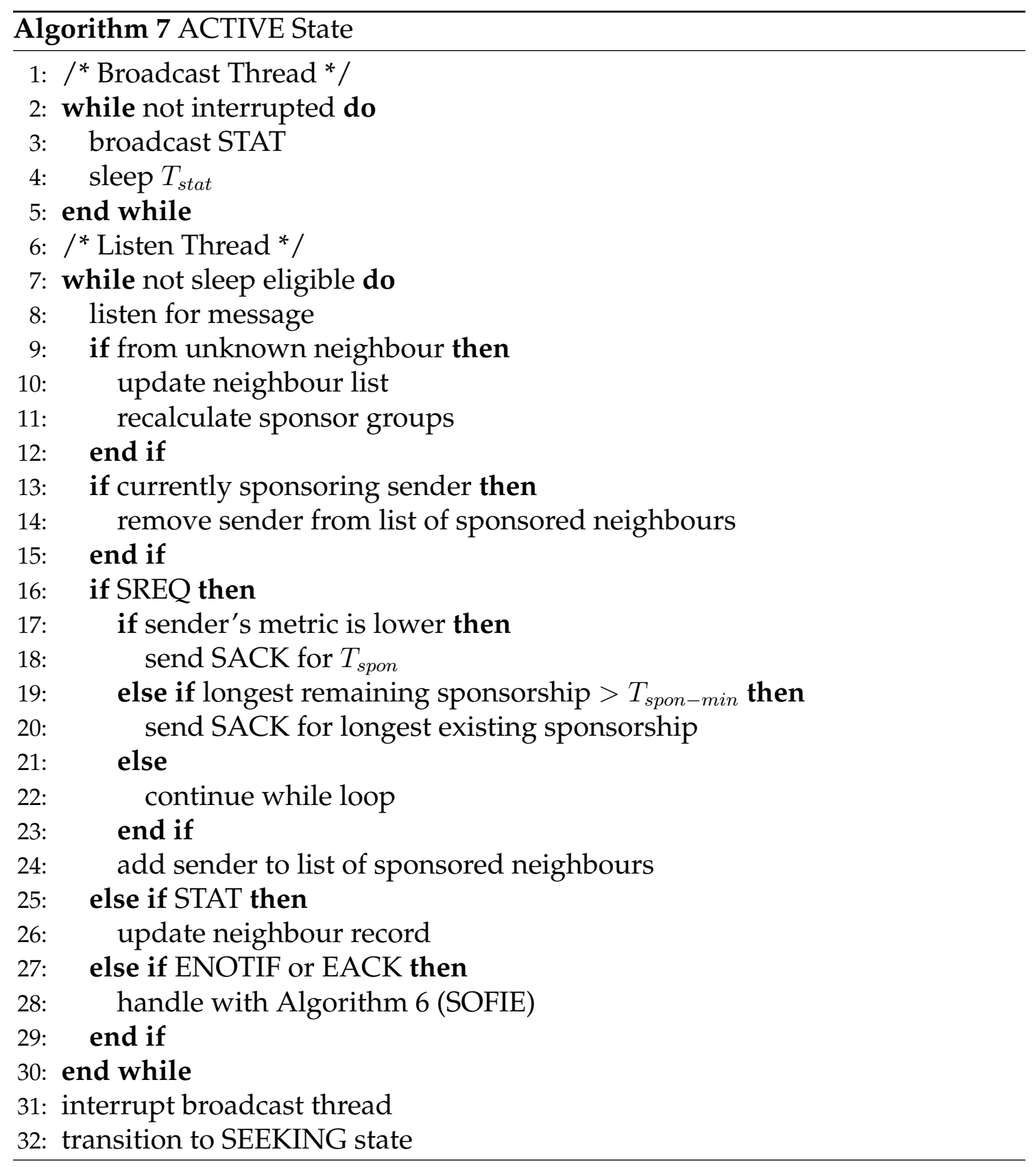


When a seeking node wishing to be sponsored has received a SACK from each of the nodes it broadcast the SREQ to, it enters SPONSORED state for a predetermined period, $T_{\text {spon }}$. In SPONSORED state, nodes enter a low energy mode where all sensors and the radio transceiver are powered down. SPONSORED state pseudocode is trivial and not shown.

If not all members of the requested sponsor group have replied with a SACK within a short time $T_{\text {sack }}$, nodes try the next most likely sponsor group. If all likely sponsor groups have been contacted with an SREQ but none have responded with sufficient SREQs, nodes transition to ACTIVE state. Pseudocode for SEEKING state is given in Algorithm 8.

If, on receipt of all expected SACKs, a node wishing to enter SPONSORED state has outstanding EACKs, that node will enter DEFERRED state for no longer than $T_{\text {spon. }}$. Whilst in DEFERRED state, if all expected EACKs are received after some elapsed time $T_{d e f}$, the node transitions to SPONSORED state for $T_{\text {spon }}-T_{\text {def }}$. If the node is still in DEFERRED state when $T_{\text {spon }}$ is exceeded, the node transitions to ACTIVE state. Pseudocode for DEFERRED state is given in Algorithm 9.

If fewer SACKs are received than expected, the requesting node reverts to ACTIVE state and periodically broadcasts STATs (cf. Algorithm 7). Neighbouring nodes that had already agreed to be sponsors remove the requesting node from their sponsored lists on receipt of any of these STATs. On wake-up from a period in SPONSORED state, nodes similarly enter ACTIVE state, their sponsoring nodes adjusting their sponsored lists accordingly on receipt of these STATs.

SREQs contain the metric (sleep \% or stored charge) of the requesting node (cf. Section 6.3.5). Potential sponsor nodes in ACTIVE or SEEKING state but not currently sponsoring any neighbours will respond to the SREQ with a SACK if and only if the sponsoring node has a higher metric than the requester. If the requesting neighbour's metric is higher, a SACK is returned for whatever time is left on the longest existing sponsorship, unless that time is less then $T_{\text {spon-min }}$ in which case the SREQ is ignored. 


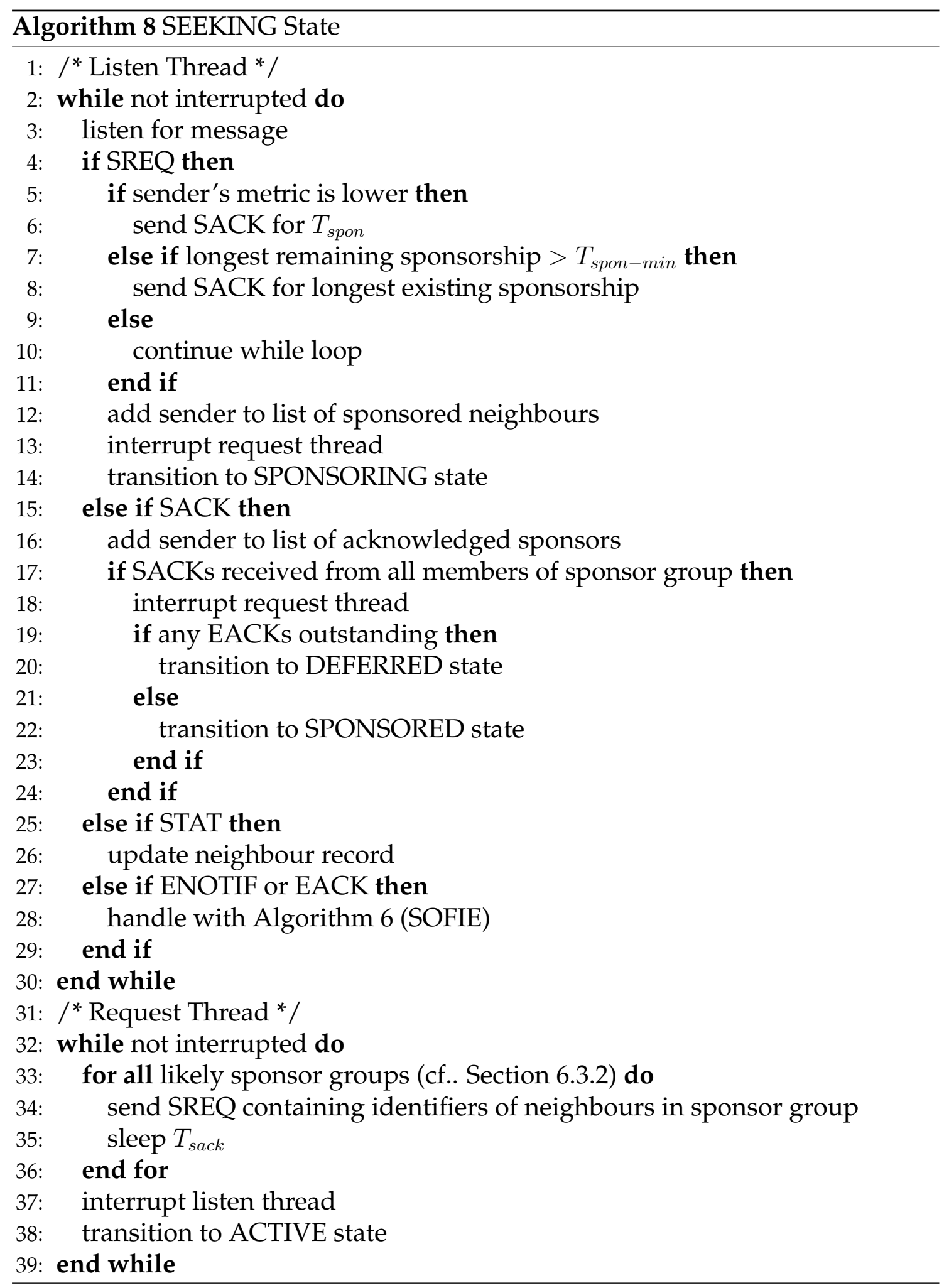




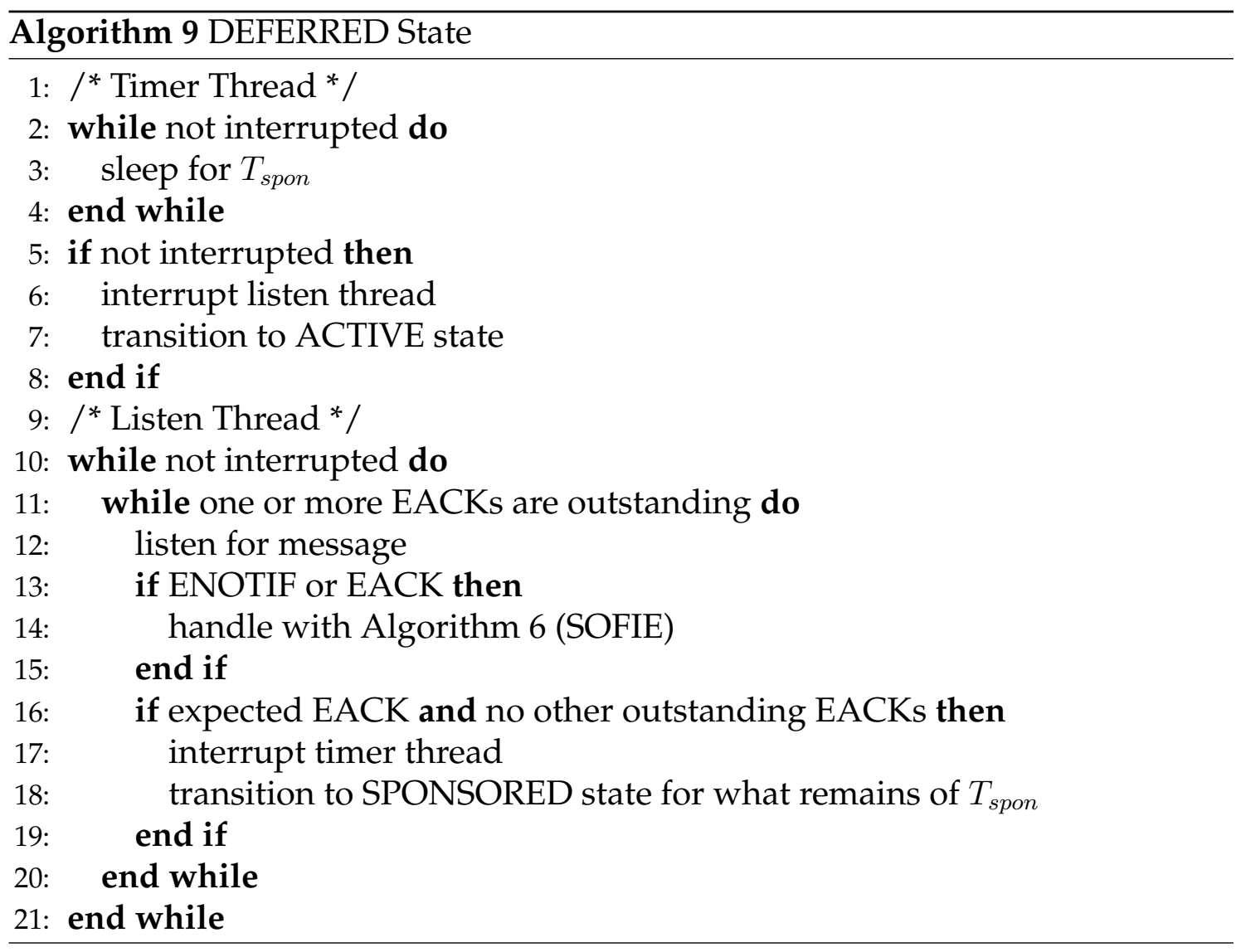




\subsubsection{The Sleep Eligibility "Daisy Chain"}

As CPRF allows nodes in SPONSORING state to seek sponsors of their own, a "daisy chain" effect arises in dense deployments where nodes in SPONSORING state continue to seek and find sponsors of their own. Table 6.1 shows an example of this situation.

Table 6.1: Sleep Eligibility - Daisy Chaining

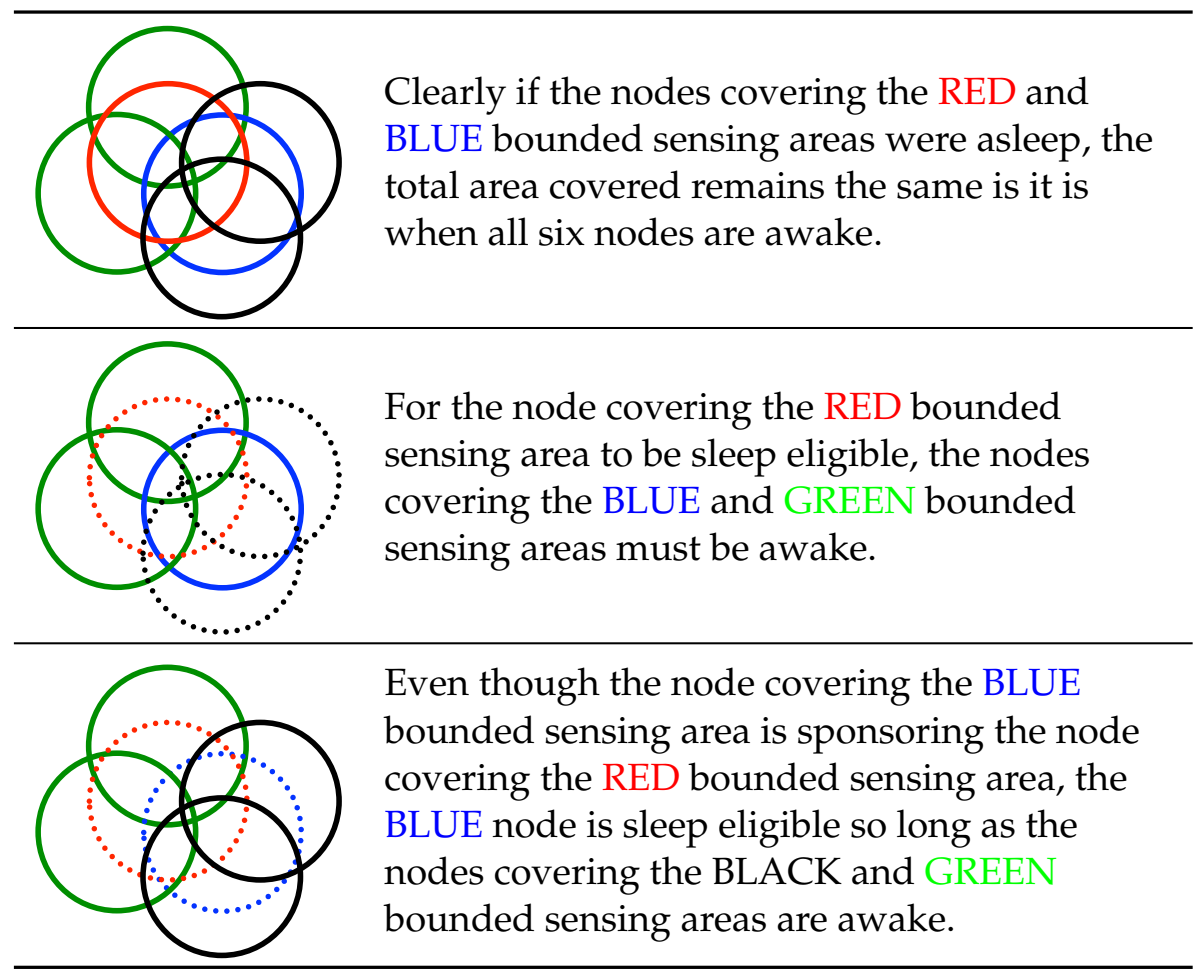

Dotted boundary indicates node is asleep 


\subsection{Evaluation}

Two deployment scenarios are used to evaluate CPRF through simulation and implementation on commercially available low power WSN devices: area coverage where an over population of nodes cover a regularly shaped polygon, and point coverage where a small number of nodes are co-located to sense events occurring on structural elements, in this case, excessive displacement of light columns.

CPRF, CCP /CBF, and 3DkCov-ComFor are implemented in QualNet 5.2 [201] with all nodes based on Advanticsys CM5000 IEEE 802.15.4 compliant motes configured as non-beaconing full-function devices with energy consumption profiles as shown in Table 3.3, CSMA/CA parameters as shown in Table 4.3, and algorithm settings as shown in Table 6.2.

Table 6.2: Algorithm Settings

\begin{tabular}{lll}
\hline CPRF & & \\
Maximum Time in Sponsored State & $T_{\text {spon }}$ & 30 minutes \\
Minimum Sponsoring Time & $T_{\text {spon-min }}$ & 30 seconds \\
Maximum Sponsor Group Size & $S G_{\text {size }}$ & 6 \\
Maximum Sponsor Groups per Node & $S G_{\text {max }}$ & 128 \\
Estimated One-hop Delay & $T_{\text {hop }}$ & $30 \mathrm{~ms}$ \\
EACK Delay & $T_{\text {eack }}$ & $60 \mathrm{~ms}$ \\
\hline CCP/CBF & & \\
Max Time in SLEEP State & $T_{\text {ccp }}$ & $30 \mathrm{mins}$ \\
Max Forwarding Delay & $T_{\text {cbf }}$ & $45 \mathrm{~ms}$ \\
\hline 3D-kCov-ComFor & & \\
Round Duration & $T_{\text {round }}$ & $30 \mathrm{mins}$ \\
Listening Time & $T_{\text {listen }}$ & 3 secs \\
\hline
\end{tabular}

\subsubsection{Sleep Eligibility}

As deployment densities increase for fixed sensing and communication ranges, more nodes become sleep eligible. CPRF allows nodes sponsoring others to enter SPONSORED state so long as doing so does not reduce sensing coverage. ESCARGO does no have this ability and in dense deployments will realise a lower proportion of sleeping nodes. 
In a $50 \mathrm{~m}^{2}$ sensing area with $R_{s}=20 \mathrm{~m}, R_{c}=44 \mathrm{~m}$ hexagonal deployments of increasing density from near perfect to heavily over populated are generated and allowed to duty cycle for a simulated 48 hours for both ESCARGO and CPRF. Figure 6.3 charts the mean number of sleeping nodes from 100 uniquely seeded simulations. CPRF clearly allows an increasing large number of nodes to sleep as density increases.

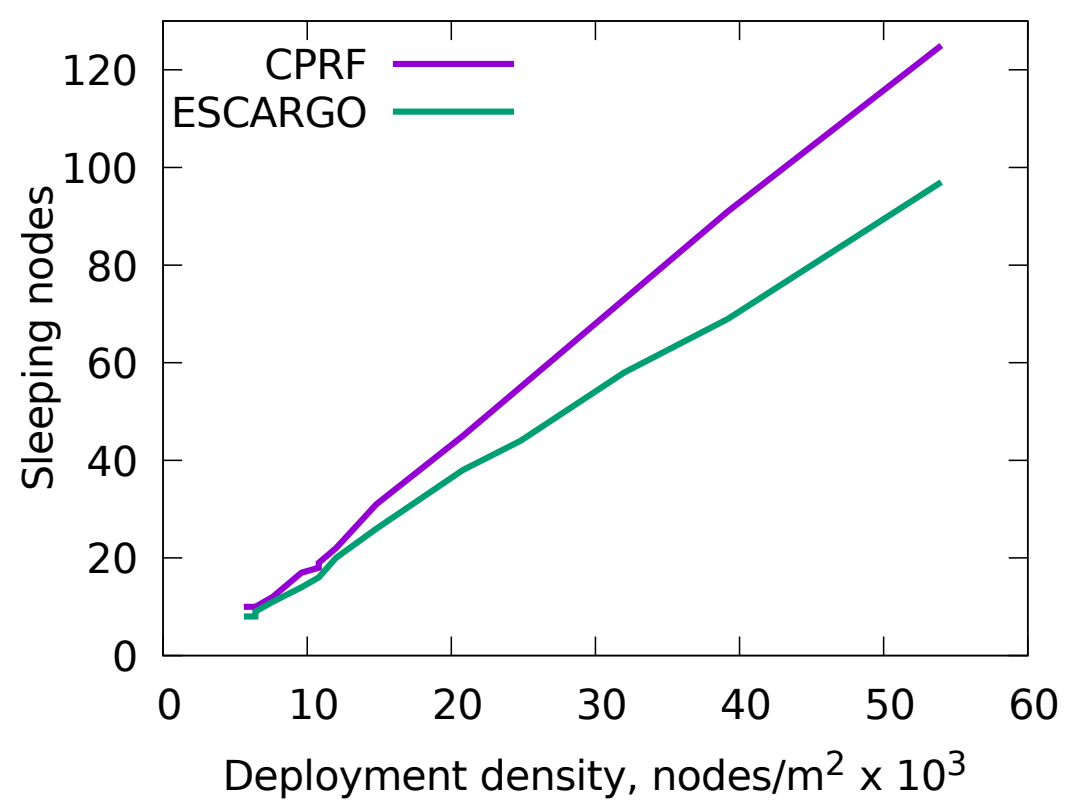

Figure 6.3: Sleeping nodes by increasing deployment density. $50 \mathrm{~m}^{2}$ square sensing area, increasing density from perfect to heavily over populated.

\subsubsection{Coverage Preservation}

Efficacy of the CPRF coverage preservation algorithm is assessed by comparison with $\mathrm{CCP} / \mathrm{CBF}$ and $3 \mathrm{D}-\mathrm{kCov}-\mathrm{ComFor}$. The $\mathrm{CCP}$ coverage preservation component of $\mathrm{CCP} / \mathrm{CBF}$ is a mature algorithm with similar aims to CPRF in terms of minimising the number of active sensing nodes whilst maintaining sensing coverage. CCP does not, however, have a mechanism for guaranteeing coverage or for ensuring nodes share sleep opportunities equitably. Similarly, 3D-kCov-ComFor has no mechanism for ensuring coverage is maintained, but it does enforce a rule that all nodes must sleep at least every third round.

In an energy harvesting WSN intended for uninterrupted continual deployment, it is inevitable that the node allowed to sleep the least will experience the 
most severe dips in stored energy. Equally, if care is not taken to ensure nodes transitioning to a sleeping state do not leave coverage gaps, critical events occurring in those uncovered areas will go undetected. Our comparative analysis therefore concentrates on each algorithm's ability to (a) maintain perfect sensing coverage, (b) maximise the sleep time of the node(s) worst placed to spend time asleep, and (c) allow all nodes to maintain sufficient reserves of stored charge in order not to "die" during periods of low harvestable energy.

Figure 6.4 charts coverage for a 116-node deployment at ten times the deployment density required for complete coverage of the sensing area.

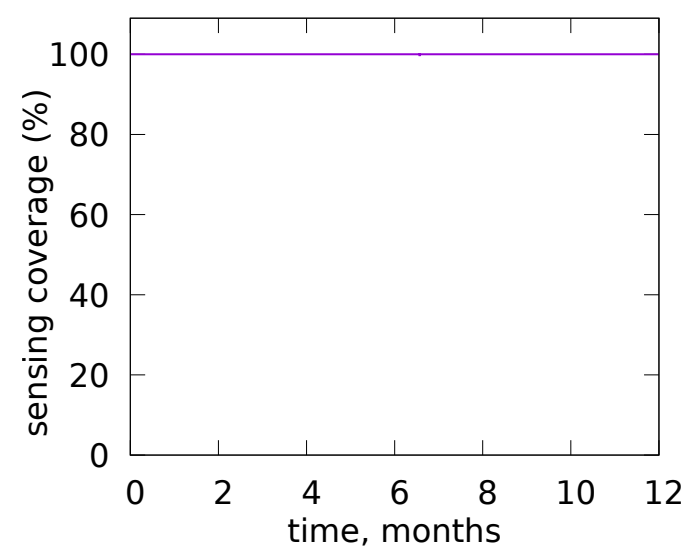

(a) 3D-kCov-ComFor

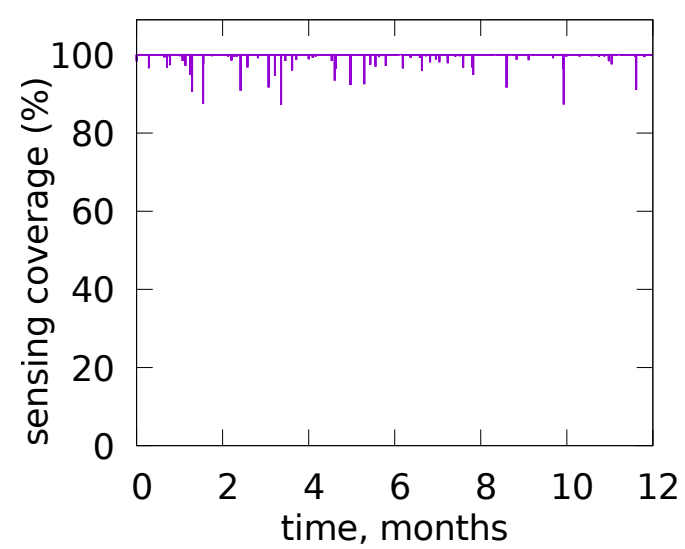

(b) $\mathrm{CCP} / \mathrm{CBF}$

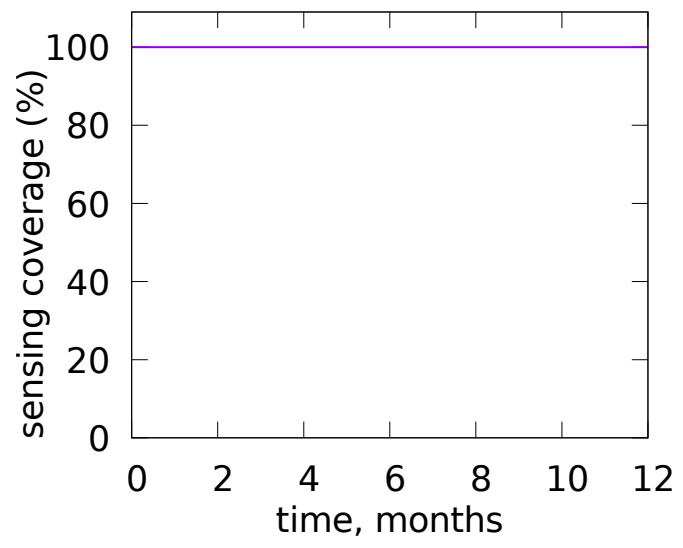

(c) CPRF

Figure 6.4: Sensing Coverage in Dense Deployment: 116 nodes, one year, ten times min deployment density, 50\% charging efficiency, CPRF metric $=$ charge.

Whilst CPRF and 3D-kCov-ComFor maintain perfect coverage throughout the simulated year, $\mathrm{CCP} / \mathrm{CBF}$ frequently looses coverage, occasionally by as much as $14.7 \%$. CCP/CBF is unable to guarantee coverage as its sleep scheduling algo- 
rithm is based on a local understanding of whether or not neighbours are awake. In these evaluations, $\mathrm{CCP} / \mathrm{CBF}$ performs an invalid sleep $0.4 \%$ of the time. $\frac{1}{4}$ of these invalid sleep result in losses of coverage ranging from $0.01 \%$ to $14.7 \%$.

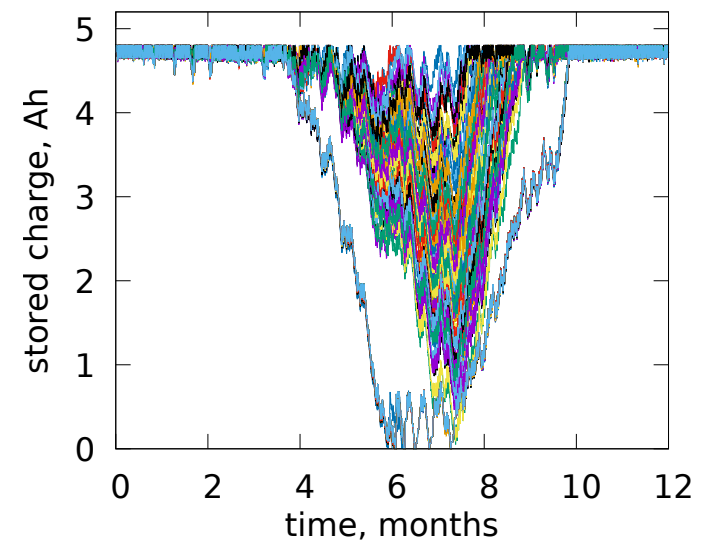

(a) 3D-kCov-ComFor

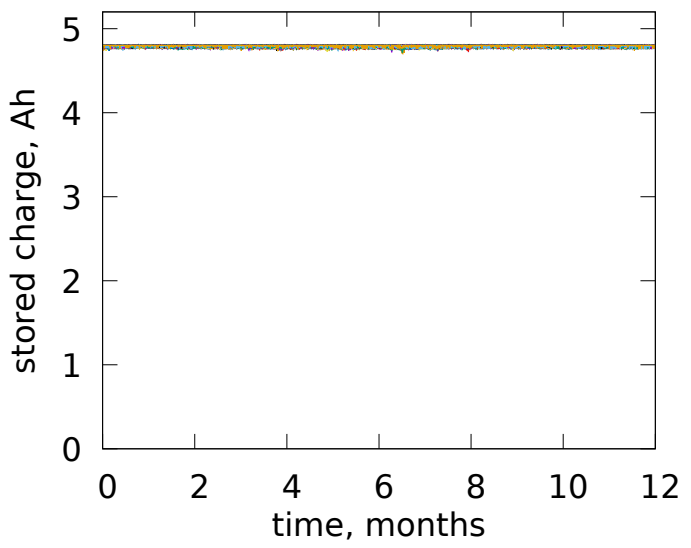

(b) $\mathrm{CCP} / \mathrm{CBF}$

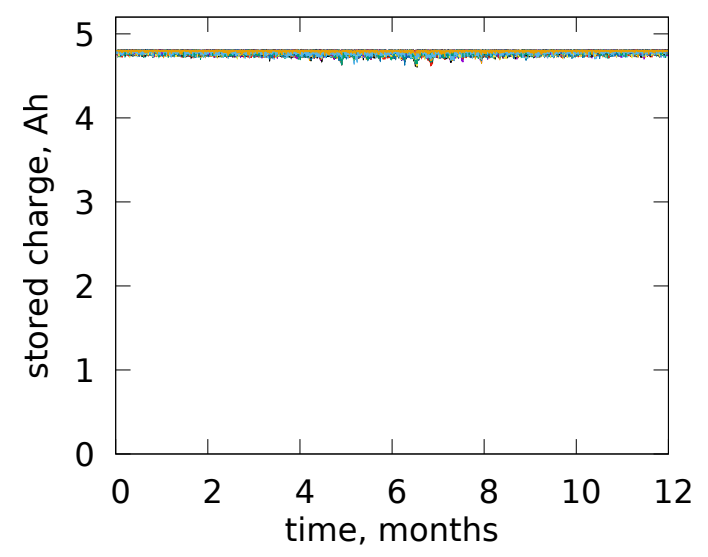

(c) CPRF

Figure 6.5: Stored Charge in Dense Deployment: 116 nodes, one year, ten times min deployment density, $50 \%$ charging efficiency, $\mathrm{CPRF}$ metric $=$ charge.

$\mathrm{CCP} / \mathrm{CBF}$ nodes check their sleep eligibility using Theorem 2 and an understanding of their neighbours sleep status based on previously received messages. $\mathrm{CCP}$ regularly broadcasts beacon messages, so rarely believes a neighbour is asleep when it is actually awake. Conversely, when a CCP/CBF node is about to go to sleep, it broadcasts a single "withdraw" message to that effect. As wireless communications are inherently unreliable, not all these sleep notification messages will be received by interested neighbours. Subsequent sleep eligibility assessments may be flawed as a neighbour believed to be awake may actually be asleep. 


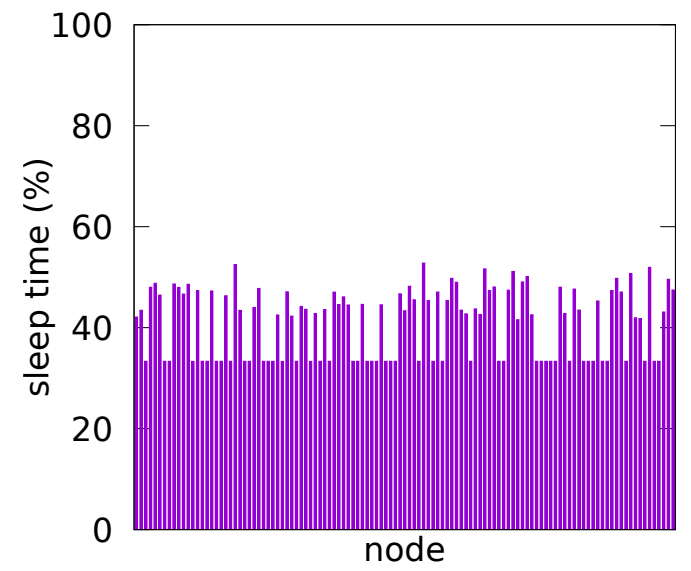

(a) 3D-kCov-ComFor

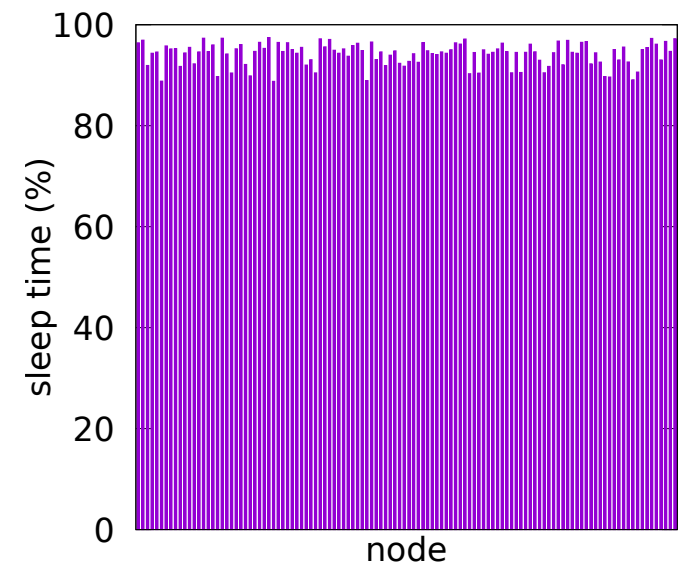

(b) $\mathrm{CCP} / \mathrm{CBF}$

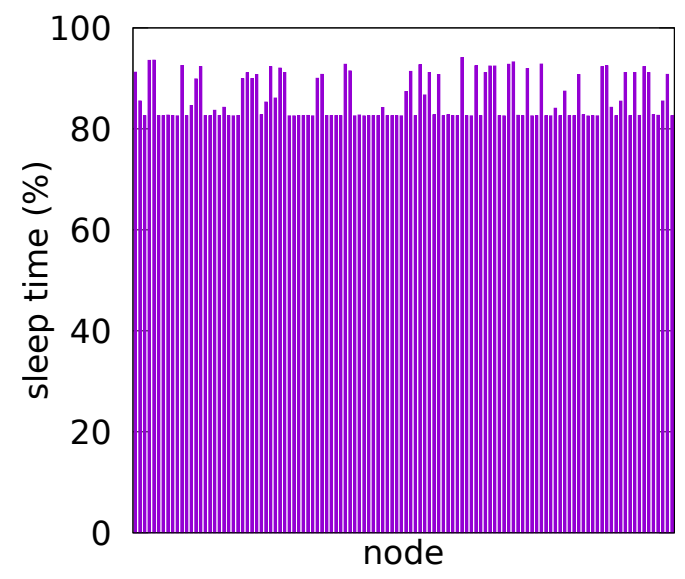

(c) CPRF

Figure 6.6: Sleeptime in Dense Deployment: 116 nodes, one year, ten times min deployment density, 50\% charging efficiency, $\mathrm{CPRF}$ metric $=$ charge.

CPRF nodes also store what they believe to be the sleeping state of their neighbours, but are able to guarantee coverage as they will not enter SPONSORED state (i.e. go to sleep) unless they have explicit acknowledgement from all members of the selected sponsor group that they are (a) actually awake and (b) willing to stay awake until the requesting node wakes up. Whilst the CPRF SACK is just as likely to go unreceived as a CCP "withdraw" message, CPRF takes a fail safe approach and will not allow a node to sleep unless all expected SACKs have been received.

In addition to guaranteeing coverage is maintained at all times, CPRF strives to give nodes equal opportunity to enter SPONSORED state, i.e. equal opportunity to go to sleep. CCP contains no logic to monitor sleep equality, relying 


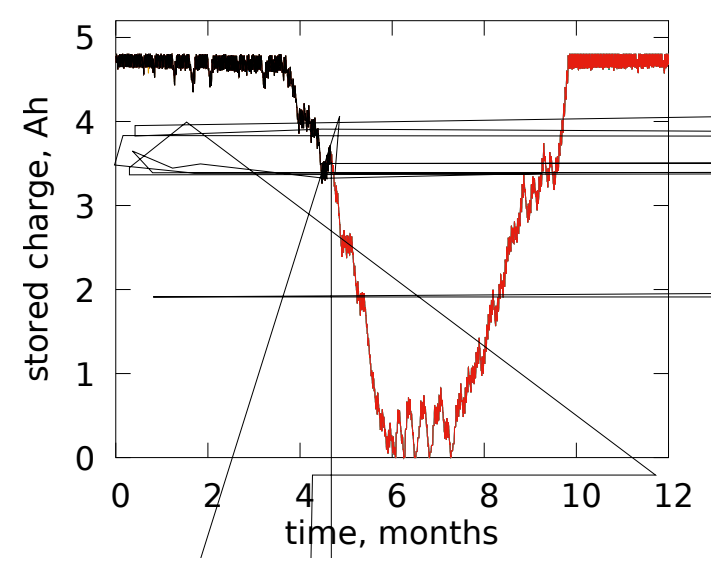

(a) 3D-kCov-ComFor

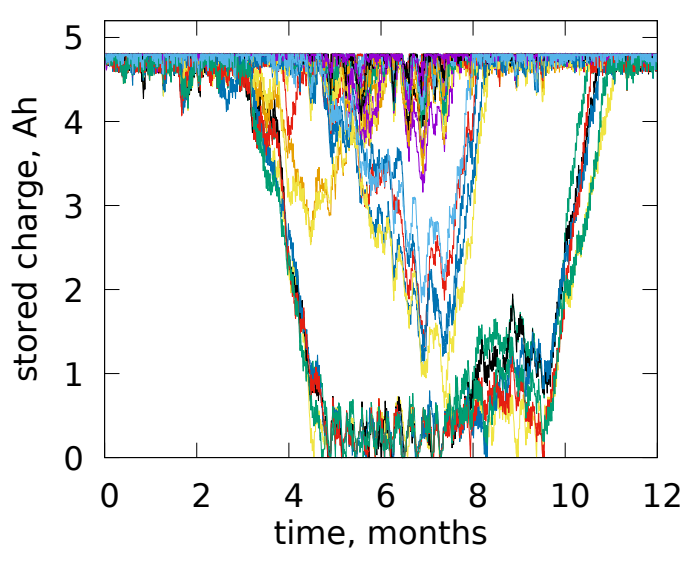

(b) $\mathrm{CCP} / \mathrm{CBF}$

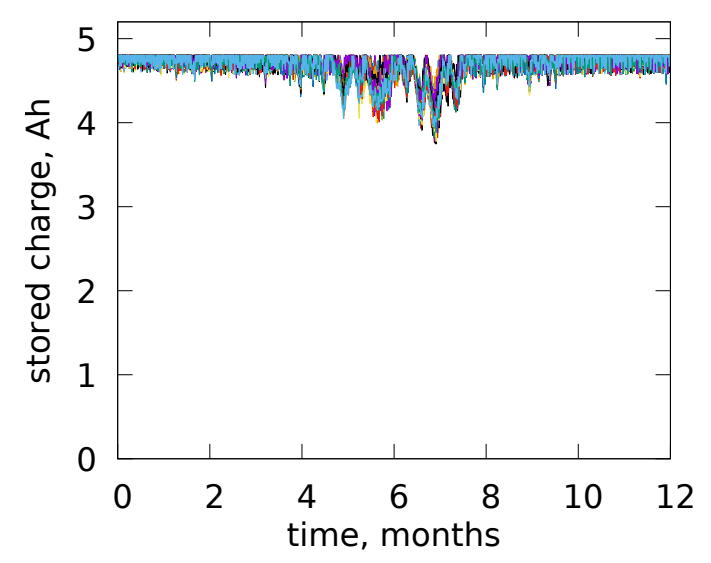

(c) CPRF

Figure 6.7: Stored Charge in Sparse Deployment: 116 nodes, one year, ten times min deployment density, 50\% charging efficiency, $\mathrm{CPRF}$ metric $=$ charge.

instead on randomised timer lengths to allow nodes in less favourable situations to enter a sleeping state. It should be noted that CCP was not designed for energy harvesting WSNs intended for continuous operation, rather its aim is to extend the operational life of battery powered WSNs by maximising the time during which some level of coverage is preserved; sleep equality therefore not being a necessary requirement.

In sparse deployments, some CCP/CBF nodes, particularly those located on or near the sensing area boundary, rarely find themselves sleep eligible; in CPRF terms they have few sponsor groups, often only one, but more significantly, their neighbours in that small number of sponsor groups have a comparatively large set of sponsor groups, many of which contain the poorly situated nodes themselves. 


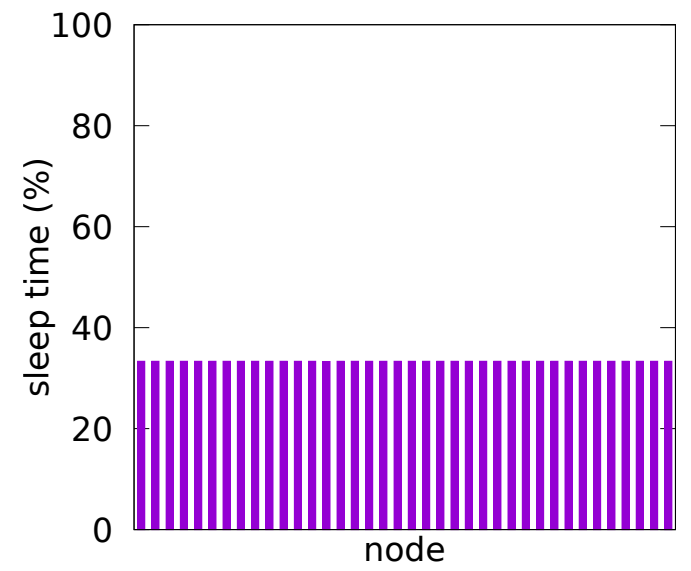

(a) 3D-kCov-ComFor

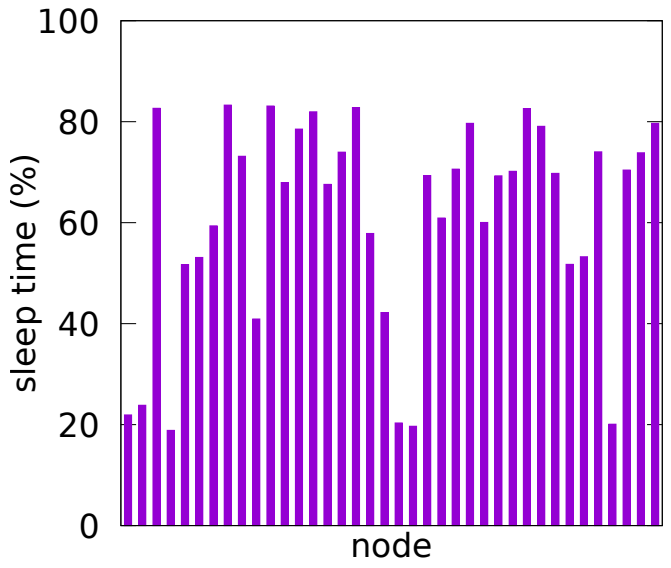

(b) $\mathrm{CCP} / \mathrm{CBF}$

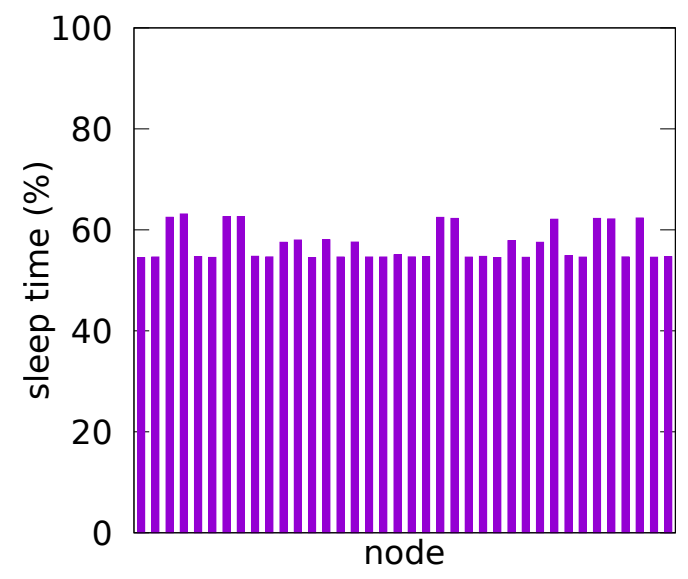

(c) CPRF

Figure 6.8: Sleeptime in Sparse Deployment: 116 nodes, one year, ten times min deployment density, 50\% charging efficiency, $\mathrm{CPRF}$ metric $=$ charge.

Figure 6.8 charts the sleep time for 38 nodes at minimum deployment density over a full calendar year. Whilst CPRF approximately equalises sleep time for all nodes regardless of their location in the sensing area, a significant number of CCP nodes spend considerably less time asleep than their neighbours. All 3DkCov-ComFor nodes spend exactly $\frac{1}{3}$ of their time asleep. As nodes never assess themselves to be sleep-eligible, this leads to the network constantly switching from $100 \%$ to $0 \%$ coverage every $2 T_{\text {round }}$ and back to $100 \%$ after $T_{\text {round }}$ as shown in Figure 6.9(a).

In energy harvesting WSNs, unequal sleep times can have a dramatic effect on the network's ability to maintain coverage and connectivity during periods when little energy is available for harvesting. Figures 6.7(a)(b)(c) chart stored charge in 


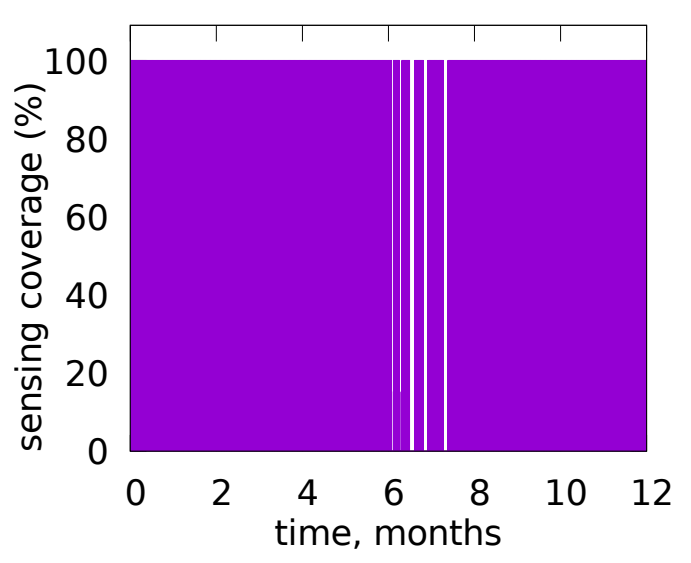

(a) 3D-kCov-ComFor

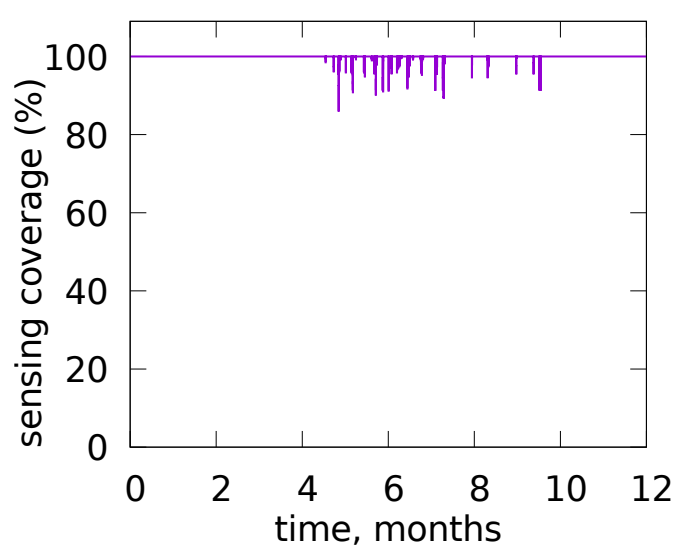

(b) $\mathrm{CCP} / \mathrm{CBF}$

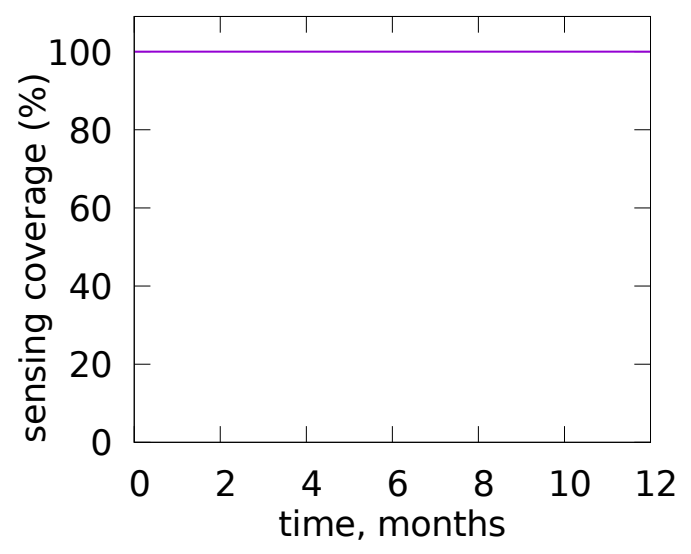

(c) CPRF

Figure 6.9: Sensing Coverage in Sparse Deployment: 116 nodes, one year, ten times min deployment density, 50\% charging efficiency, CPRF metric $=$ charge.

the network of 38 nodes at minimum deployment density over a full calendar year.

CPRF's ability to equalise sleep times gives all nodes the ability to easily replenish their stored charge from solar energy even during the short days of winter. 3D-kCov-ComFor nodes are time synchronised and sleep exactly the same amount of time on exactly the same schedule. Figure 6.7(a) clearly shows all nodes exhaust their reserves of stored charge at the same time and likewise recover simultaneously.

Whilst many CCP nodes survive the winter as easily as they would if running CPRF, those that infrequently find themselves sleep eligible experience a rapid decline in their stored charge as winter approaches. During the winter months the $\mathrm{CCP} / \mathrm{CBF}$ nodes that sleep the least repeatedly run out of energy and have to 


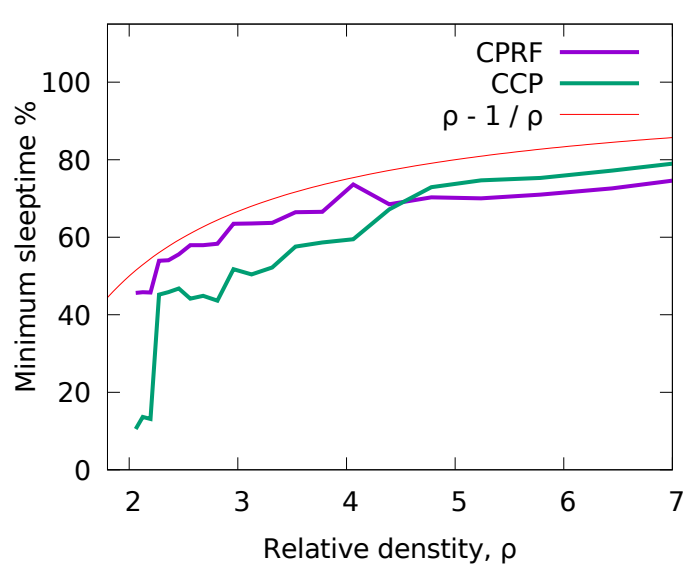

(a)

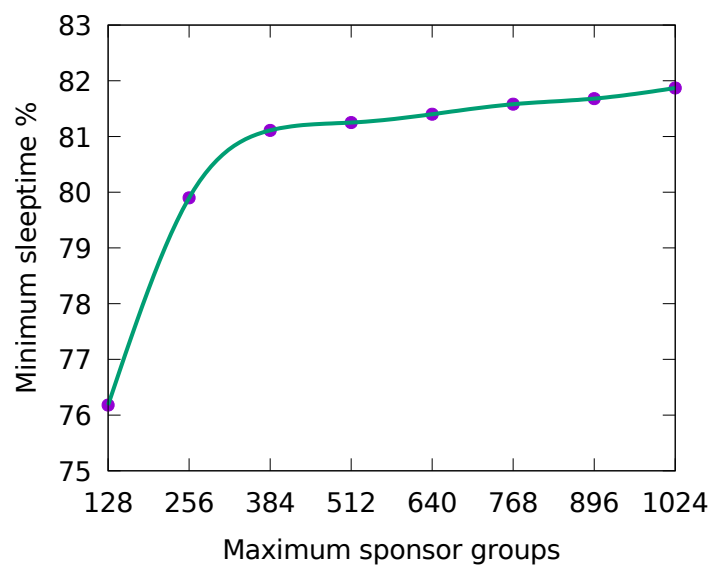

(b)

Figure 6.10: (a) Minimum sleeptime by increasing deployment density $\rho$, where $\rho=1$ is the minimum density required for complete sensing coverage, and $\rho=2$ is minimum density required for each node's sensing area to be 1-covered by its neighbours. (b) Minimum sleep time for CPRF by increasing $S G_{\max }$ for $\rho=4.86$.

wait until more has been harvested before they can re-join the sensor network.

As CCP/CBF nodes exhaust their reserves of stored charge their extended absences from the network force other nodes to stay awake more often, depleting their energy reserves more rapidly than if their neighbours were energised and able to share coverage responsibility.

At cost-effective low deployment densities, CPRF's focus on sleep time equality allows it to maintain a consistently higher minimum sleep time than that achieved by CCP. At higher deployment densities, the gap narrows, but CPRF maintains its advantage until the point where more sponsor groups exist than a CPRF node has the ability to record in core memory.

Figure 6.10(a) charts minimum sleep time against deployment density when CPRF is restricted to a maximum of 128 sponsor groups per node. Figure 6.10(b) shows how increasing the maximum number of sponsor groups each node can maintain $\left(S G_{\max }\right)$ for a given deployment density provides no significant increase in minimum sleep time beyond a certain point. If the evaluation in Figure 6.10(a) where re-run with $S G_{\max }$ set to a higher value, the cross-over point where CCP delivers a higher minimum asleep time than CPRF would occur at a higher deployment density.

In finite state simulations increasing $S G_{\max }$ is of little consequence, the additional memory and processing time required being of easy to accommodate on the sizeable machines on which the simulations are run. However, on limited 
resource WSN devices where RAM is extremely limited and computation cycles are both power hungry and slow, restricting the number of neighbours and the number of sponsor groups that must be kept track of becomes necessary. As a consequence, at high deployment densities, practical implementations of CCP will often provide a marginally better least sleep time, but they cannot guarantee coverage is maintained at all times.

To summarise this coverage preservation evaluation, we considered two scenarios:

Sparse Deployments CPRF maintains perfect sensing coverage, equalises sleep time at around 50\%, and all nodes retain sufficient stored charge to survive periods of low harvestable energy. $\mathrm{CPP} / \mathrm{CBF}$ is unable to equalise sleep times and consequently some nodes are unable to maintain sufficient stored charge to survive periods of low harvestable energy resulting in regular loses of sensing coverage. 3D-kCov-ComFor equalises sleep time at exactly

$\frac{1}{3}$ but looses sensing coverage entirely for the same proportion of time as all nodes sleep simultaneously. Increased levels of coverage loss are seen during periods of low harvestable energy.

Dense Deployments CPRF again maintains perfect sensing coverage equalises sleep time to the extent that the node that sleeps least spends over $80 \%$ of its time asleep. Nodes easily survive periods of low harvestable energy. $\mathrm{CBB} / \mathrm{CBF}$ does a marginally better job of equalising sleep times with the node that sleeps least spending over $90 \%$ of its time asleep. However, sensing coverage loses of up to $14 \%$ are regularly seen. $3 \mathrm{D}-\mathrm{kCov}-\mathrm{ComFor}$ also maintains perfect sensing coverage and equalises sleep times well but the nodes that sleep least are only asleep $30 \%$ of the time. In addition, many nodes exhaust their reserves of stored charge during periods of low harvestable energy.

\subsubsection{Rapid Forwarding}

CPRF's rapid forwarding capability is provided by an integrated implementation of SOFIE [4]. Assessment of this integration's efficacy is undertaken in 116-node simulated rectangular networks of decreasing sensing area, $R_{c}$ and $R_{s}$. Deployment density is such that approximately half the nodes can be in SPONSORED state at any given time. 
A network sink is placed in one corner of the sensing area and events are generated pseudo-randomly in the opposite corner at intervals between three and four times $T_{\text {spon }}$. This randomised event generation process affects (a) which nodes in range of the event are awake and will therefore sense it, and (b) which nodes between between the event sensing node(s) and the sink are awake. The latter introduces non-determinism in the forwarding paths of notification messages (ENOTIFs) and their returned acknowledgements (EACKS). In some cases, nodes sensing the events will have to enter DEFERRED state whilst waiting for an EACK.

Table 6.3 shows aggregated results from 100 distinctly seeded evaluations for minimum, mean, and maximum notification delay, number of hops taken, percentage of notifications arriving without requiring a retry, and the percentage (\%) of transitions to SPONSORED state that went via DEFERRED state.

Table 6.3: Detection Delay for Randomly Located Events

\begin{tabular}{lccccccccc}
\hline & & & & & & \multicolumn{4}{c}{ Notif. Delay (ms) } \\
Nodes & Area & $R_{c}$ & $R_{s}$ & 1st Time & Deferred & Hops & Min & Mean & Max \\
\hline 116 & $360 \times 360$ & $100 \mathrm{~m}$ & $40 \mathrm{~m}$ & $99.5 \%$ & $0.007 \%$ & 3.78 & 2.8 & 16.4 & 615 \\
116 & $270 \times 270$ & $75 \mathrm{~m}$ & $30 \mathrm{~m}$ & $99.7 \%$ & $0.003 \%$ & 4.14 & 2.8 & 17.3 & 436 \\
116 & $180 \times 180$ & $50 \mathrm{~m}$ & $20 \mathrm{~m}$ & $99.6 \%$ & $0.003 \%$ & 3.85 & 2.8 & 16.8 & 387 \\
\hline
\end{tabular}

Comparison is now made between CPRF, CPP/CBF and 3D-kCov-ComFor. All three algorithms restrict their forwarding regions to a sub section of the Maximum Forwarding Area (MFA) [214]. Figure 6.11 illustrate differences between the three algorithms in a randomly distributed network. CBF has a large number of nodes in its Reuleaux Triangle forwarding area, but only one will self-select as the best forwarding candidate. CPRF uses a clipped Reuleaux Triangle for its forwarding area, reducing contention between potential forwarders, although in this case several nodes will simultaneously forward any received message.

$3 \mathrm{D}-\mathrm{kCov}-\mathrm{ComFor}$ restricts its forwarding area to the $R_{s}$ radius $20^{\circ}$ Reuleaux Lens oriented towards the sink. In the case illustrated, the comparatively small forwarding region of 3D-kCov-ComFor allows only one node to act as a relay. In this scenario, 3D-kCov-ComFor would need to forward each message as many as five times more often than required by either CCP/CBF or CPRF. Further, in sparse deployments, 3D-kCov-ComFor is unlikely to find any nodes appropriately located to act as relays. 


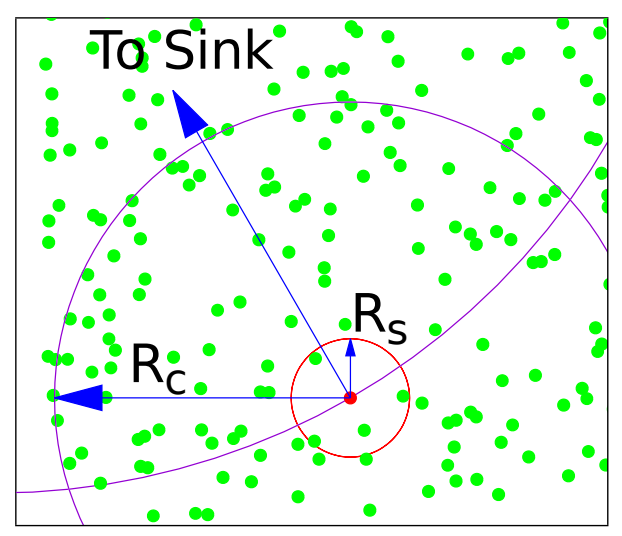

(a) Geometry

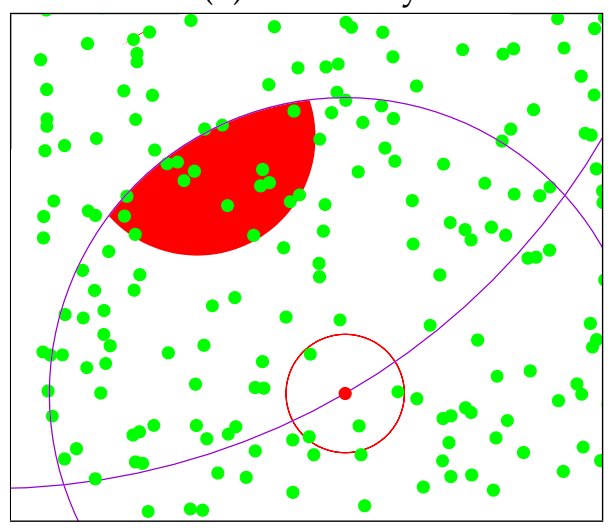

(c) CPRF

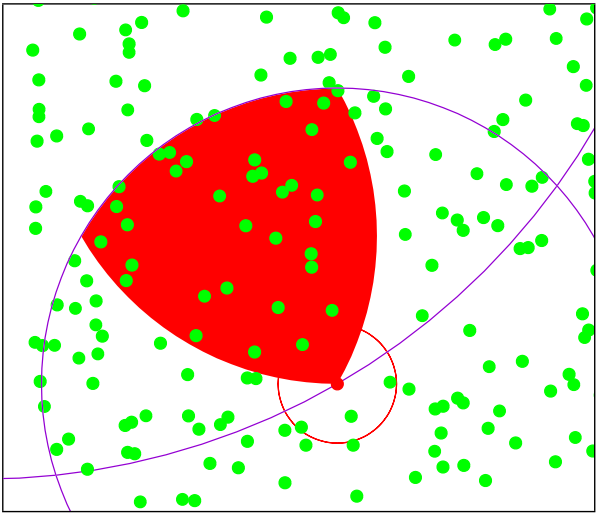

(b) $\mathrm{CCP} / \mathrm{CBF}$

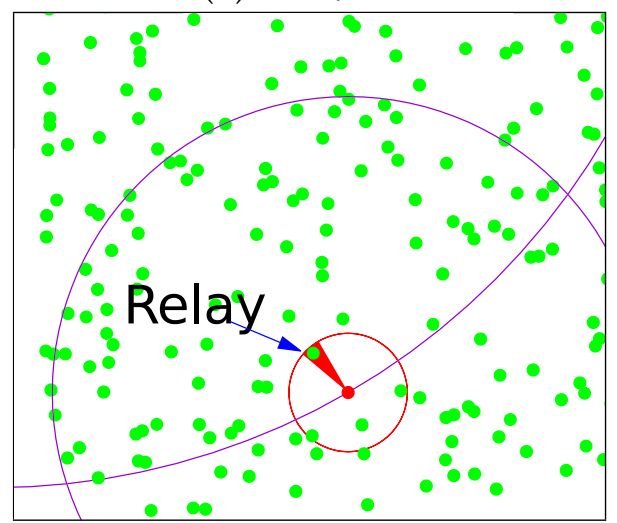

(d) 3D-kCov-ComFor

Figure 6.11: Comparison of (shaded) forwarding regions for $R_{c}=5 R_{s}$, random node placement.

Three deployment scenarios are used for comparison:

Sparse Planned placement of sufficient nodes to allow each to discover sleep opportunities as the result of being 1-covered according to Theorem 2. Deployment is twice the minimum density required to 1-cover the entire sensing area. 423 nodes in a sensing area of $20,000 \mathrm{~m}^{2}$.

Dense Random placement of ten times as many nodes as would be required to 1 -cover every point in the sensing area. 1,330 nodes in $7,000 \mathrm{~m}^{2}$.

Point Clusters of two nodes are co-located at evenly separated locations such as would be experiencd if monitoring highway light poles. Each pair of nodes monitors a single pole. 100 nodes, two per pole, inter-pole separation $20 \mathrm{~m}$. For CPRF and CCP $/ \mathrm{CBF} R_{s}=1 m$, for 3D-kCov-ComFor $R_{s}=22 m$.

In sparse and dense deployment scenarios the sensing area is significantly wider 
than it is tall. In all cases a network sink is located at one end of the sensing area. 1,000 events are generated at the opposite end of the sensing area at a frequency twenty times more rapid than the 3D-kCov-ComFor round duration $T_{\text {round }}$ (cf. Table 6.2.) Three metrics are of interest:

Delivery Ratio Critical event sensing requires all notification messages are successfully delivered. The extent to which each examined algorithm satisfies this requirement is of prime importance.

Notification Delay Assuming all messages are delivered successfully, rapid delivery is desirable for critical events. Less rapid notification is acceptable if necessary to achieve a perfect delivery ratio.

Forwarding Hops The number of hops a message takes in being delivered is not of prime interest in itself. However, capturing message propagation hops facilitates reasoning about discrepancies in notification delay between algorithms.

One hundred uniquely random seeded simulations are undertaken for each algorithm in each of the three selected deployment scenarios.

Figure 6.12(a) charts delivery ratio as a percentage. CPRF successfully delivers all event notification messages in all deployment scenarios. $\mathrm{CCP} / \mathrm{CBF}$ presents no delivery guarantees and drops a small number of messages in all deployment scenarios, though in the point deployment this is less than $0.1 \%$. In the sparse deployment 3D-kCov-ComFor fails to deliver any messages. This is expected as the is designed for a dense deployment scenario. However, even in the dense deployment scenario, 3D-kCov-ComFor still only manages to deliver $\frac{2}{3}$ of the generated messages. In the point distribution, 3D-kCov-ComFor achieved a mean successfully delivered ratio of $92 \%$.

Weighted mean delivery delay is charted in Figure 6.12(b). CPRF achieves the lowest delivery delay in all scenarios. CCP /CBF is in the order of $30 \%$ to $50 \%$ slower, but this is to be expected as CBF is designed to sacrifice propagation speed in favour of reduced collisions. In scenarios where $3 \mathrm{D}-\mathrm{kCov}-\mathrm{ComFor}$ sucessfuly delivers messages, significantly higher delivery delays are experienced. In the dense deployment scenario this is in excess of an order of magnitude slower than both CPRF and CCP/CBF. This excessive delay can be attributed to the restricted forwarding region shown in Figure 6.11(d) that results in the significantly higher hop counts shown in Figure 6.12(c). 


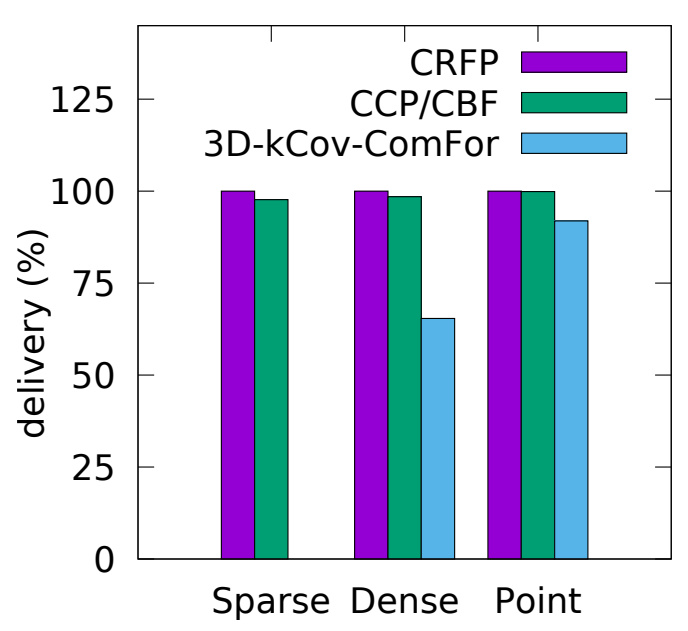

(a)

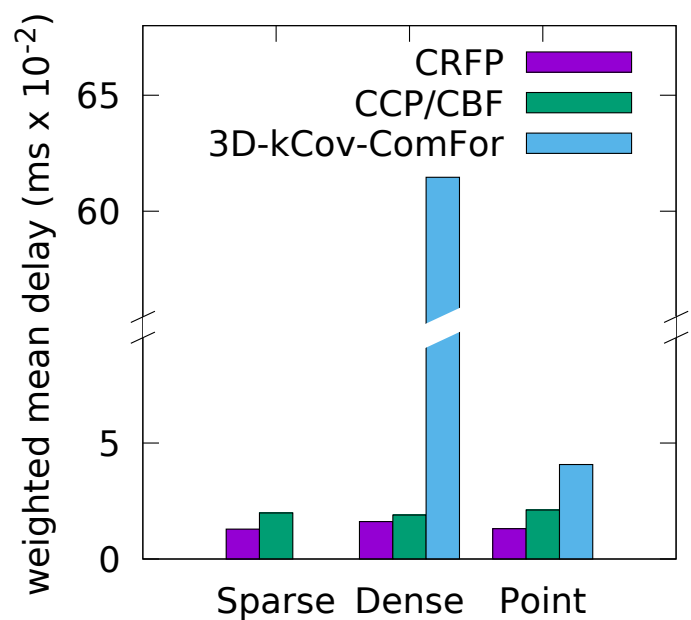

(b)

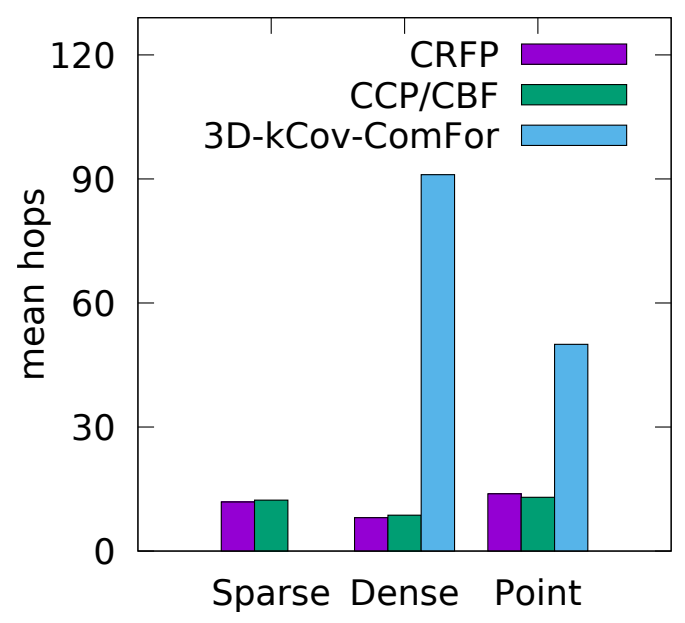

(c)

Figure 6.12: Delivery ratio, notification delay, and forwarding hops for sparse, dense, and point distributions. 


\subsection{Implementation : Test Bed \& Live Deployment}

CPRF is implemented in nesC / TinyOS 2.1.2 [104] and an existing 58-node indoor testbed, Figure 4.13, is used to evaluate the implementation. Devices are pre-programmed with their location and ignore messages from non-neighbours that, in a realistic deployment, they would not hear. In the testbed, Advanticsys CM5000 devices broadcast packets with a transmission power of $-25 \mathrm{dbm}$ giving an approximate range of $5 \mathrm{~m}$.

With $T_{\text {spon }}=60 \mathrm{~s}$ and $T_{h o p}=50 \mathrm{~ms}, 18$ nodes are given coordinates so they form clusters of three co-located nodes at six discrete locations spaced $4 \mathrm{~m}$ apart at increasing distance from a network sink. ENOTIF's are generated by a node placed $4 m$ beyond the node cluster furthest from the sink. Events are generated pseudo-randomly in the sixty seconds surrounding each three minute boundary. Event generation stops when 1,000 ENOTIFs have been broadcast, which is approximately two days after the evaluation starts.

Note that the nodes duty cycle approximately three times as frequently as they are required to forward an event notification message. Also note that in this configuration, each cluster of three CPRF nodes sleep schedule only with each other, each one having a single sponsor group containing its two co-located neighbours. Except when negotiating which node will sleep next, only one node in each cluster is awake at any given time.

All ENOTIFs are received successfully, the majority at the first attempt. Figure 6.13a shows the notification delay distribution for all messages, Figure 6.13b the distribution of the notification delay for those that arrived without requiring a re-send by the sensing node.

Outdoor assessment is initially undertaken with clusters of two co-located CPRF nodes placed on perspex stands, $1 \mathrm{~m}$ above level ground such that the distance between the clusters can be modified, as shown in Figure 6.14a. Further outdoor assessment is undertaken at two sports fields with tall light poles similar to those used on highways. Figure $6.14 \mathrm{~b}$ shows one of the sports fields used for evaluation. Such light poles are vulnerable to adverse weather conditions, like vortex shedding, as shown in Figure 1.1.

The two nodes closest to the network sink are UCMote Minis (one is shown in Figure 6.15) drawing power from the simple energy harvesting circuit shown in Figure 6.16. The UCMote Minis have an on-board charging circuit for their LIR2450 cells and were deployed fully charged to demonstrate heterogeneous 
144 CHAPTER 6. COVERAGE PRESERVATION WITH RAPID FORWARDING

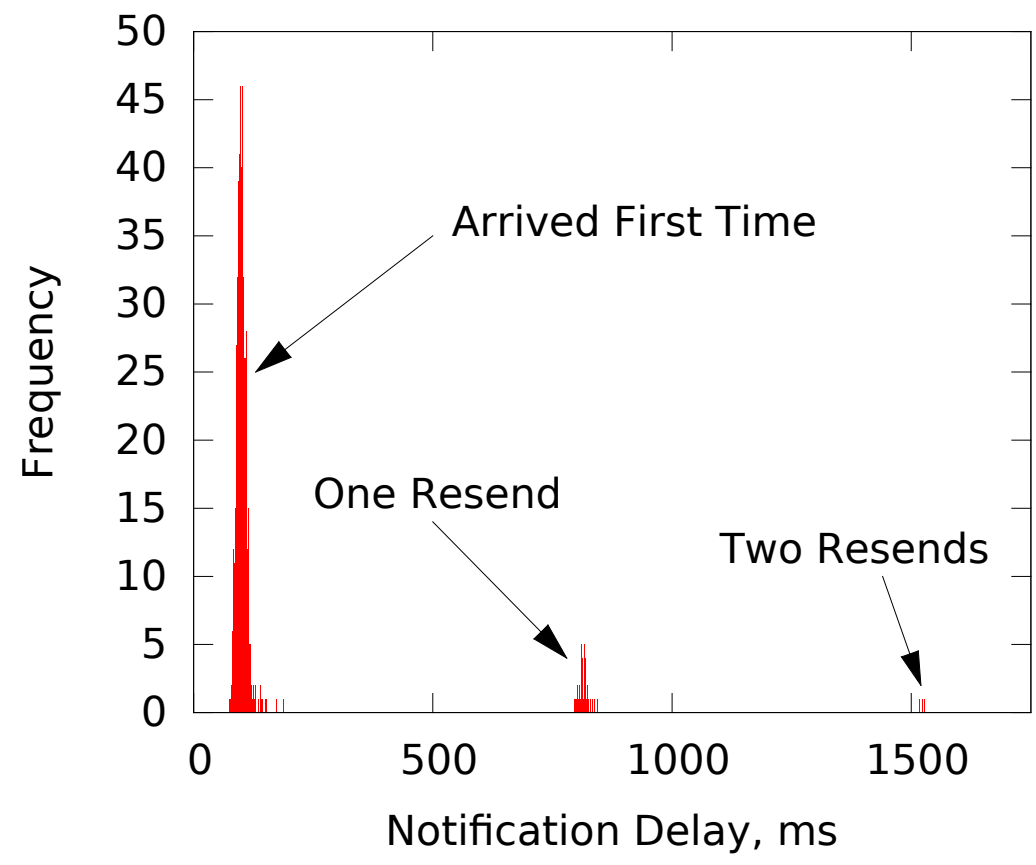

(a) None, one, \& two resends

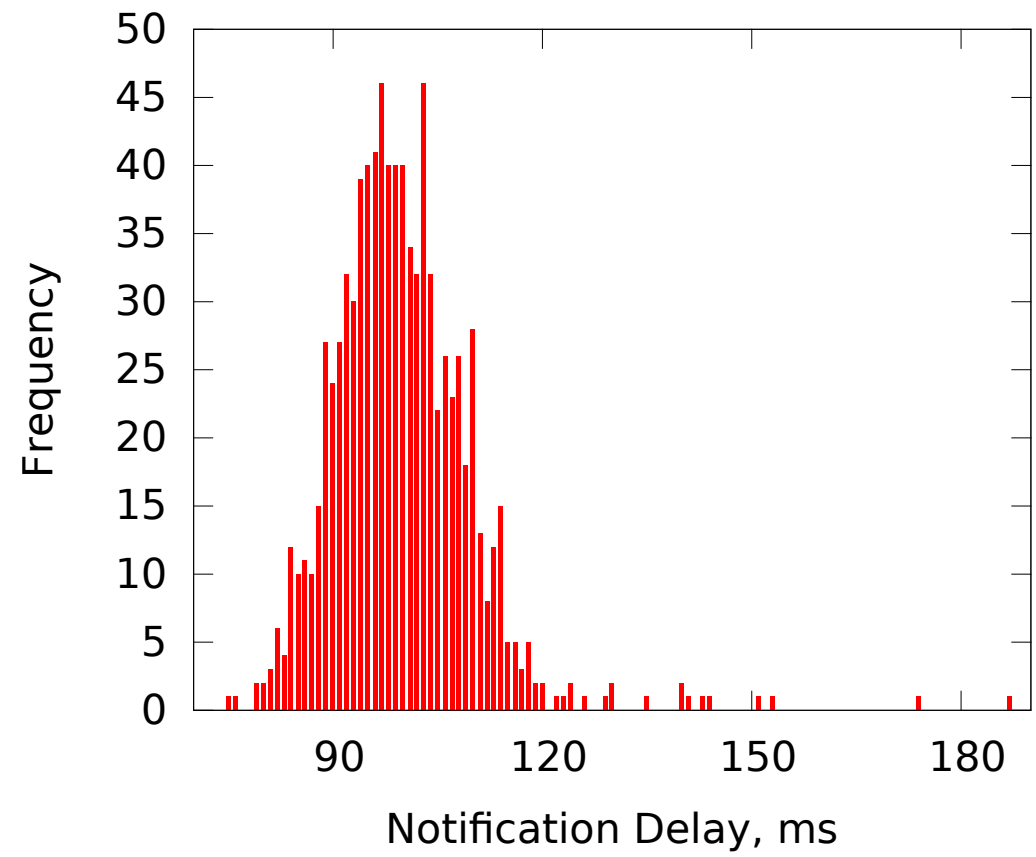

(b) No resend

Figure 6.13: Notification delay distribution: Testbed, 7 hops, 1,000 messages. 


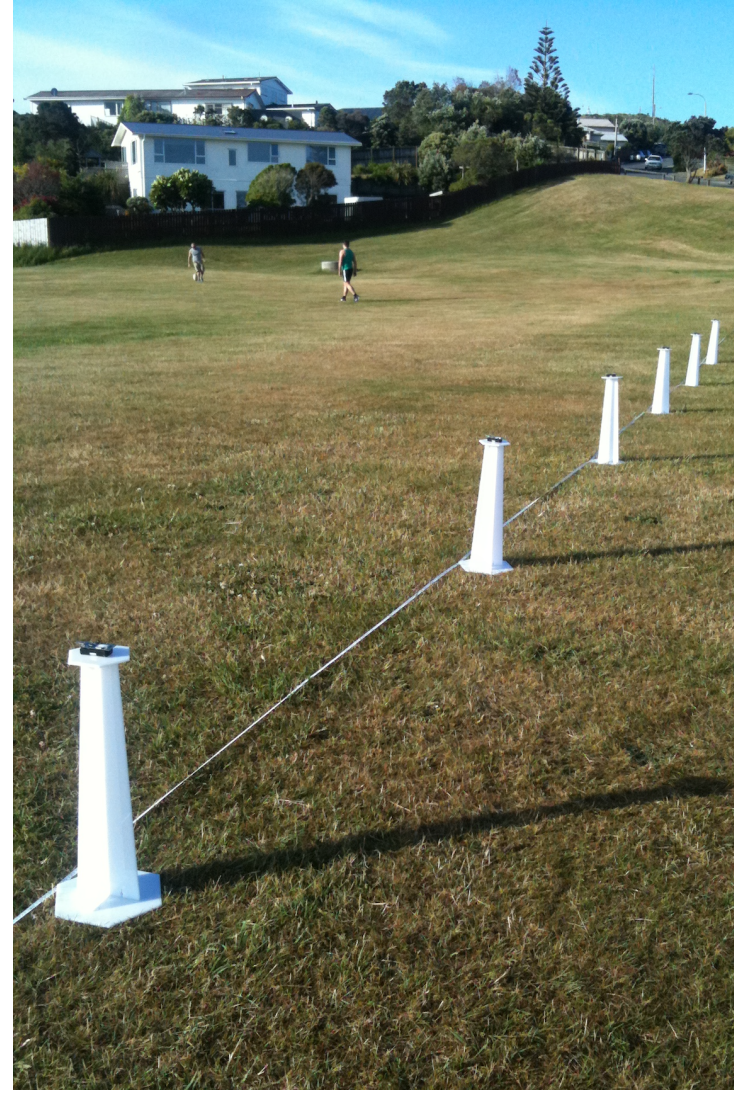

(a) Adjustable Separation

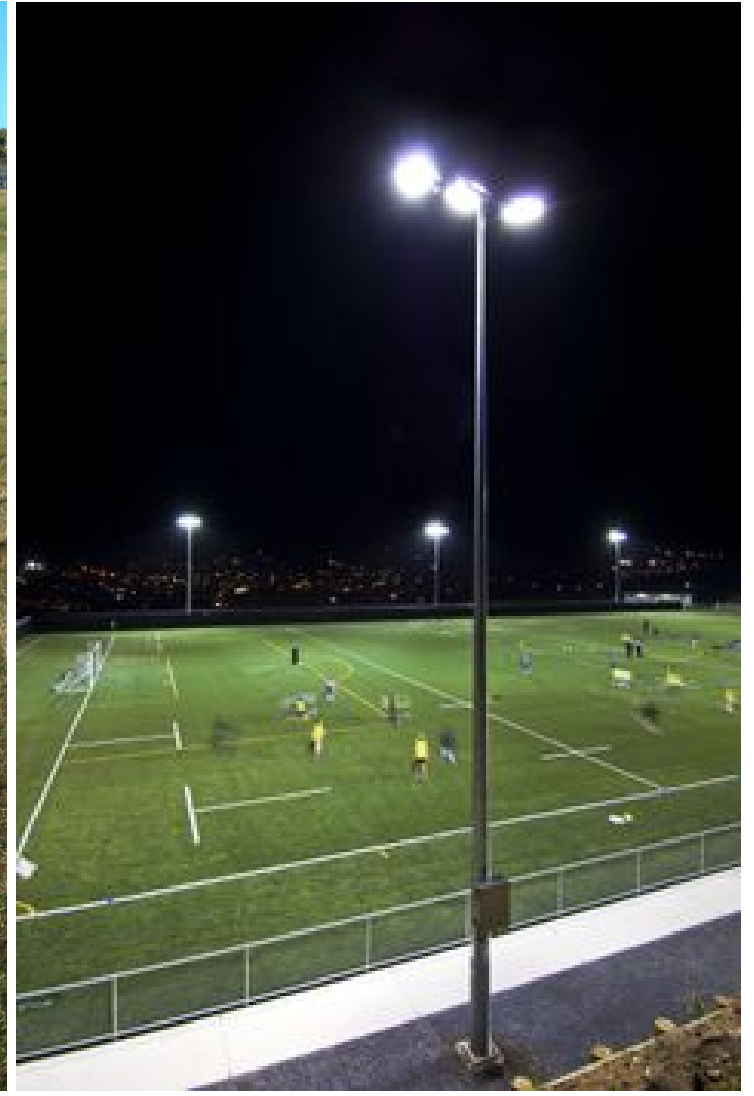

(b) Three Light Poles (4 Hops)

Figure 6.14: Evaluation Environments

operation of CPRF nodes from different manufacturers rather than mission critical energy harvesting. As neither the UCMote Mini nor Advanticsys CM5000 provide an mechanism for determining stored charge, the CPRF metric (cf. Section 6.3.5) is the percentage (\%) time asleep.

Minimum, mean, and maximum notification delay, plus mean hops taken during forwarding and the percentage of notifications that arrived without requiring a resend, for indoor testbed, outdoor assessment on stands, and two sports grounds with distinct flood light configurations are shown in Table 6.4. 
146 CHAPTER 6. COVERAGE PRESERVATION WITH RAPID FORWARDING

Table 6.4: Implementation Evaluation Results for 1,000 Messages

\begin{tabular}{|c|c|c|c|c|c|c|c|c|}
\hline \multirow[b]{2}{*}{ Config. } & \multirow[b]{2}{*}{ Poles } & \multirow[b]{2}{*}{ Separation $^{1}$} & \multirow[b]{2}{*}{$R_{c}$} & \multirow[b]{2}{*}{ 1st Time } & \multirow{2}{*}{$\begin{array}{l}\text { Mean } \\
\text { Hops }\end{array}$} & \multicolumn{3}{|c|}{ Notif. Delay (ms) } \\
\hline & & & & & & Min & Mean & $\operatorname{Max}$ \\
\hline Testbed & 2 & - & $5 \mathrm{~m}$ & $95.6 \%$ & 3.0 & 46 & 59 & 378 \\
\hline Testbed & 6 & - & $5 \mathrm{~m}$ & $87.6 \%$ & 7.0 & 75 & 126 & 1,527 \\
\hline Stands & 6 & $3.0 \mathrm{~m}$ & $5 \mathrm{~m}$ & $97.2 \%$ & 6.9 & 71 & 113 & 676 \\
\hline Floodlights 1 & 4 & $12.5 \mathrm{~m}$ & $20 \mathrm{~m}$ & $93.8 \%$ & 4.9 & 52 & 101 & 547 \\
\hline Floodlights 2 & 3 & $16.7 \mathrm{~m}$ & $20 \mathrm{~m}$ & $95.2 \%$ & 4.0 & 62 & 78 & 481 \\
\hline
\end{tabular}

${ }^{1} \mathrm{n} / \mathrm{a}$ for testbed, nodes are adjacent (c.f. Figure 4.13)

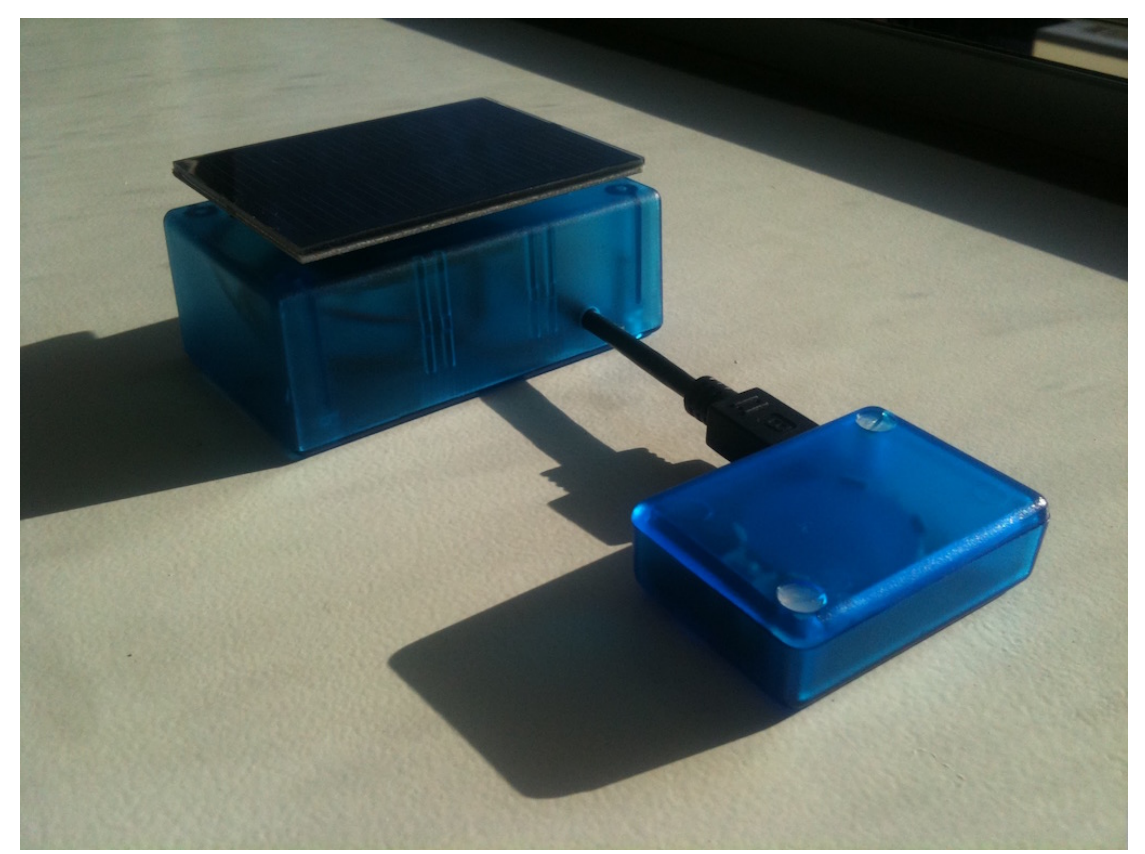

Figure 6.15: UCMote Mini (right) powered by simple photovoltaic energy harvester. USB Mini connector between the devices gives an indication of scale.

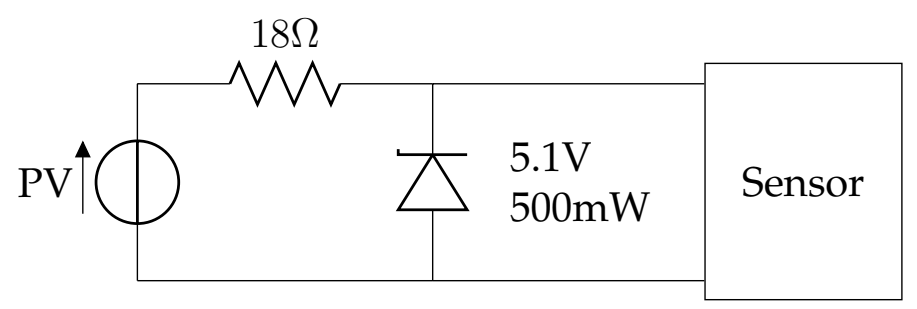

Figure 6.16: Simple photovoltaic energy harvester schematic 


\subsection{Summary}

WSNs deployed to detect critical rare events demand perfect detection probability and minimal detection delay. For detection to be guaranteed and propagation of event detection messages to be delayed only by inherent network latency, sensing coverage and network connectivity must be maintained at all times. This dual requirement is problematic for battery powered devices - a device drawing $20 \mathrm{~mA}$ will operate for just ten days, approximately, when powered by two AA batteries. On the other hand, energy harvesting is a mature technology that provides a mechanism for significantly extending the operation life of sensing devices. Large scale solar energy harvesting can easily provide enough power for a small WSN device to remain active indefinitely, but issues including cost, weight, and aesthetics may render such equipment unsuitable for specific deployment scenarios. An alternative is to use an over population of sensors each with a small scale energy harvesting capability. These devices can take turns to enter a low-power state where they take no part in sensing or communication activities of the network but have the opportunity to replenish their energy stores at a more rapid rate than when active.

In deployment scenarios where fastest possible event notification is required and losses of sensing coverage are unacceptable, our proposed Coverage Preservation with Rapid Forwarding (CPRF) scheme has been shown to provide a robust mechanism for guaranteeing all events are detected and notification messages are not only guaranteed to be delivered, but are propagated with the least possible delay. Further, CPRF has been shown to deliver more reliable coverage guarantees, and more rapid and reliable event notification than a best-of-breed composition of well established coverage and forwarding algorithms, and a recently proposed state-of-the-art integrated coverage and forwarding scheme. 
148 CHAPTER 6. COVERAGE PRESERVATION WITH RAPID FORWARDING 


\section{Chapter 7}

\section{Conclusions}

WSNs deployed to detect critical rare events demand perfect detection probability and minimal detection delay. For detection to be guaranteed and propagation of event detection messages to be delayed only by inherent network latency, sensing coverage and network connectivity must be maintained at all times. This dual requirement is problematic for battery powered devices; for example, a device drawing $20 \mathrm{~mA}$ will operate for little more than ten days when powered by a pair of AA batteries.

Conversely, energy harvesting is a mature technology that provides a mechanism for significantly extending the operation life of sensing devices. Large scale solar energy harvesting can easily provide enough power for a small WSN device to remain active indefinitely, but issues including cost, weight, and aesthetics may render such equipment unsuitable for certain deployment scenarios.

An alternative is to use an over population of sensors each with a small scale energy harvesting capability. These devices can take turns to enter a low-power state where they take no part in sensing or communication activities of the network but have the opportunity to replenish their energy stores at a more rapid rate than when active.

In deployment scenarios for critical rare events, where fastest possible event notification is required and losses of sensing coverage are unacceptable, the Coverage Preservation with Rapid Forwarding (CPRF) scheme has been shown to provide a robust mechanism for guaranteeing all events are detected and notification messages are propagated with the least possible delay.

The forwarding mechanism embedded in CPRF, Swift Opportunistic Forwarding for Infrequent Events (SOFIE), has been shown to deliver more rapid event 
notification than a well established contention based forwarding algorithm.

CPRF's coverage preservation capabilities have further been shown to outperform a well established coverage preservation algorithm in sparse planned deployment networks and beyond - where deployment density ranges from twice the minimum required to cover the sensing area to four or five times that minimum. At higher densities, those approaching random deployments, CPRF maintains perfect sensing coverage where the established competitor continues to regularly loose coverage. On low specification devices, those with limited RAM, at these higher deployment densities CPRF delivers marginally higher minimum sleep time but continues to provide total sensing coverage at all times.

\subsection{Review}

The research presented in this thesis investigates whether or not modest capability, commercially available low-power WSN devices, when powered by smallscale energy harvesting, can provide guaranteed coverage and lowest possible latency connectivity for potentially indefinitely long periods.

After surveying related work in Chapter 2, subsequent chapters build on each other, the end result being a demonstration that CPRF answers this research question in the affirmative. Chapters containing original research are summarised as follows:

Chapter 3 If finite state network simulations are to be meaningful, accurate power consumption models must be used. An experimental evaluation of two commercially available IEEE 802.15.4 WSN devices provided data for the power consumption and energy harvesting models used in subsequent chapters. Further, a number of popularly held assumptions about how small wireless devices consume energy were demonstrated to be invalid for the devices under test.

Chapter 4 The power consumption and energy harvesting models from Chapter 3 were used in finite state simulations of ESCARGO that demonstrate the algorithm is better than an existing scheme at equalising sleep time amongst sensing nodes in sparse planned and randomly deployed networks. Year round operation is shown to be achievable with small-scale solar energy harvesting at charging efficiencies within the capabilities of existing devices. A physical implementation of ESCARGO is evaluated on a medium 
scale indoor testbed and is shown to match the simulated version under battery power and a novel simulated energy harvesting scheme.

Chapter 5 With event detection assured by ESCARGO's coverage preservation capabilities, an algorithm is proposed to assure minimum detection delay by rapidly forwarding event detection messages delayed only by the media contention inherent in wireless networks. SOFIE is shown to deliver swifter message propagation than two existing geographical forwarding algorithms in networks of sparse and dense randomly and uniformly placed sensing devices. SOFIE is further shown to maintain the ability to rapidly forward messages in networks that experience the kind of rapid topology changes experienced by network running collaborative duty cycling protocols like ESCARGO and CPRF.

Chapter 6 SOFIE and the sleep equivalency focus of ESCARGO are combined into an single algorithm capable of both maintaining sensing coverage and rapidly forwarding event detection messages through the resulting unstable topology network. CPRF is shown to deliver optimum active node density whilst maintaining both sensing coverage and connectivity in unreliable networks. Comparison with a highly respected previously proposed algorithm with similar coverage preservation aims shows CPRF achieves equally good minimisation of energised nodes, but performs significantly better in coverage preservation, demonstrating perfect coverage at all times whilst the competitor repeatedly looses coverage.

\subsection{Contributions}

This thesis explored aspects of coverage and connectivity provision for WSNs deployed to detect critical rare events. Research contributions have been made in multiple areas, and are restated here from Section 1.2 for completeness. In detail, the contributions made by this thesis are that:

1. Critical rare event sensing is a research area distinct from previously well studied related topics.

2. Invalid assumptions made about energy consumption of real devices can lead to the construction of inaccurate energy models for finite state simula- 
tions. Of particular note is the potential to invalidate all network forwarding schemes based on the assumption that reducing the transmit power of a low-power radio transceiver causes it to consume less energy when transmitting packets.

3. Ensuring sleep or stored charge equivalence in coverage preserving duty cycling schemes maximises the operational life of battery powered WSNs and makes effectively indefinite operation a realistic goal when energy harvesting is adopted.

4. Ignoring traditional concerns including collision avoidance and energy conservation in geographical forwarding algorithms running on standards based MAC protocols allows maximally rapid propagation of event detection messages.

5. In forwarding algorithms that do not attempt to avoid collisions, it is shown that information fusion techniques utilising time and geometry based heuristics to suppress duplicate event notification messages are of little practical benefit.

6. Maximum detection probability and minimal detection delay can be realised in a single protocol that preserves sensing coverage and ensures a permanently connected network.

\subsection{Future Work}

The scope of the research presented in this thesis is such that a number of areas remain open for extension and further investigation:

Re-evaluation of Transmission Power Control schemes: TPC schemes have been shown invalid in the sense that their basic assumption of a reduction in transmit power results in a proportional reduction in energy consumption does not hold for a number of commercially available devices. Further research could be undertaken to determine what devices and/or classes of devices are suitable for TPC and which are not. Of those that are suitable for TPC, an evaluation could be made of the extent to which energy savings are realised by individual devices and networks as a whole. 
Event de-duplication in elongated deployments: In SOFIE and hence CPRF, event de-duplication by an information fusion technique based on an awareness of sensing node location and time of arrival at potential forwarding nodes was shown to deliver little benefit in area deployments where the mean number of hops to a network sink was in the order of no more than ten. It is possible that in elongated deployments, or any deployment where the mean hop count from sensing node to network sink is high, that event deduplication would realise some benefit.

Support for hole coverage: Real world area deployments can suffer from unavoidable coverage holes such as the "pond" problem where a body of water prevents sensor nodes being placed as we might like. SOFIE, and hence CPRF, assumes perfect area coverage with no holes; inclusion of an existing hole avoidance algorithm would be beneficial. Hole avoidance also takes care of the rare circumstance when sponsoring nodes die and those they are sponsoring have yet to wake up.

Multi-sink support: In multi-sink scenarios, SOFIE will simultaneously forward to all sinks whereas the compared algorithms and others assume a single sink per packet. Multi-sink capabilities can be simulated in algorithms that embed the destination location in their messages by having the application layer initiate a message for each sink. A comparison of which technique is more effective is of interest.

Comparison with flooding algorithms: Practical WSNs, especially those designed for extended lifespans that make them suitable candidates for CPRF, can expect to require software upgrades during their lifetime. Flooding protocols exist for this task [191] and an investigation of to what extent flooding and rapid message dissemination can be combined may prove beneficial to rare event sensing networks.

Energy efficiency has long been the focus of WSN research [121, 122]. A comparatively recent study [220] specifically surveys energy conservation techniques for WSNs. It is therefore reasonable to assume that for a given sensing task, innovative techniques that demonstrably reduce energy consumption while maintaining an appropriate level of sensing functionality and network connectivity will continue to be in demand. 
As an increasing number of wireless devices are deployed under the IoT banner, distinctions between network types (sensor, actuator, ad-hoc, body-area) may become blurred and more about deployment scenarios than equipment and protocols. Should IoT drive a convergence of underlying technologies and the standardisation of low cost, energy efficient programmable wireless devices, multipurpose networks rather than point solutions may become the norm. Such a convergence of device use and network traffic could cause a significant degradation in rare event detection delay unless the system as a whole honours QoS rules requiring critical rare event notification messages to be transmitted with high priority.

Regardless of the multi/single purpose nature of future deployments, it appears reasonable to predict the critical rare event algorithms presented in this thesis will remain relevant in appropriate circumstances, yet the accuracy of their assumptions may change over time. The requirement for over-population, for example, may become less prevalent if rechargeable battery and charging technology improves significantly in capacity and efficiency; the ability to store more energy more quickly potentially negating the need to duty cycle nodes to satisfy the demands of energy hungry transceivers. 


\section{Bibliography}

[1] D. C. Harrison, W. K. G. Seah, and R. Rayudu, "Coverage preservation in energy harvesting wireless sensor networks for rare events," in Proceedings of the 40th Annual IEEE Conference on Local Computer Networks", Clearwater Beach, USA, 26-29 Oct 2015.

[2] D. C. Harrison, W. K. G. Seah, and R. Rayudu, "Rare event detection and propagation in wireless sensor networks," ACM Computing Surveys (CSUR), vol. 48, no. 4, p. 58, 2016.

[3] D. C. Harrison, D. Burmester, W. K. G. Seah, and R. Rayudu, "Busting myths of energy models for wireless sensor networks," Electronics Letters, vol. 52, no. 16, 2016.

[4] D. C. Harrison, H. Yu, W. K. Seah, and R. Rayudu, "Opportunistic geographic forwarding in wireless sensor networks for critical rare events," in Proceedings of the 41st Annual IEEE Conference on Local Computer Networks", Dubai, UAE, 7-10 Oct 2016.

[5] W. Ye, J. Heidemann, and D. Estrin, "Medium access control with coordinated adaptive sleeping for wireless sensor networks," IEEE/ACM Transactions on Networking, vol. 12, no. 3, pp. 493-506, 2004.

[6] C. Schurgers and M. B. Srivastava, "Energy efficient routing in wireless sensor networks," in Proceedings of Military Communications Conference (MILCOM), Vienna, Virginia, USA, 28-31 Oct 2001, pp. 357-361.

[7] Y. Yao and J. Gehrke, "Query processing in sensor networks." in Proceedings of the First Biennial Conference on Innovative Data Systems Research, Asilomar, CA, USA, 2003, pp. 233-244.

[8] J. E. Elson, "Time synchronization in wireless sensor networks," Ph.D. dissertation, University of California, Los Angeles, 2003. 
[9] G. Rubino and B. Tuffin, Rare event simulation using Monte Carlo methods. John Wiley \& Sons, 2009.

[10] P. Glasserman, P. Heidelberger, P. Shahabuddin, and T. Zajic, "Multilevel splitting for estimating rare event probabilities," Operations Research, vol. 47, no. 4, pp. 585-600, 1999.

[11] J. Bucklew, Introduction to rare event simulation. Springer Science \& Business Media, 2013.

[12] S. Misra, S. Mishra, and M. Khatua, "Social sensing-based duty cycle management for monitoring rare events in wireless sensor networks," IET Wireless Sensor Systems, vol. 5, no. 2, pp. 68-75, 2015.

[13] L. Xu, J. Cao, X. Liu, H. Dai, and G. Chen, “EODS: An energy-efficient online decision scheme in delay-sensitive sensor networks for rare-event detection," in 18th International Conference on Parallel and Distributed Systems (ICPADS). IEEE, 2012, pp. 307-314.

[14] S.-P. Cheon, S. Kim, S.-Y. Lee, and C.-B. Lee, “Bayesian networks based rare event prediction with sensor data," Knowledge-Based Systems, vol. 22, no. 5, pp. 336-343, 2009.

[15] S. Asur and S. Parthasarathy, "Correlation-based feature partitioning for rare event detection in wireless sensor networks," in First International Workshop on Knowledge Discovery from Sensor Data, Sensor-KDD, San Jose. Citeseer, 2007.

[16] P. Dutta, M. Grimmer, A. Arora, S. Bibyk, and D. Culler, "Design of a wireless sensor network platform for detecting rare, random, and ephemeral events," in Proceedings of the 4th international symposium on Information processing in sensor networks. Los Angeles, California, USA: IEEE Press, 2005, p. 70 .

[17] Q. Cao, T. Abdelzaher, T. He, and J. Stankovic, "Towards optimal sleep scheduling in sensor networks for rare-event detection," in Proceedings of the 4th International Symposium on Information Processing in Sensor Networks (IPSN), Los Angeles, CA, USA, 25-27 Apr 2005, p. 8. 
[18] A. Arora, P. Dutta, S. Bapat, V. Kulathumani, H. Zhang, V. Naik, V. Mittal, H. Cao, M. Demirbas, M. Gouda, Y. Choib, T. Hermanc, S. Kulkarnid, U. Arumugamd, M. Nesterenkoe, A. Vorae, and M. Miyashitae, "A line in the sand: A wireless sensor network for target detection, classification, and tracking," Computer Networks, vol. 46, no. 5, pp. 605-634, 2004.

[19] K. S. Low, W. N. N. Win, and M. J. Er, “Wireless sensor networks for industrial environments," in Proceedings of International Conference on Computational Intelligence for Modelling, Control and Automation, and International Conference on Intelligent Agents, Web Technologies and Internet Commerce, vol. 2. Vienna, Austria: IEEE, 2005, pp. 271-276.

[20] E. Gelenbe and E.-H. Ngai, "Adaptive QoS routing for significant events in wireless sensor networks," in Proceedings of 5th IEEE International Conference on Mobile Ad Hoc and Sensor Systems. Atlanta, GA, USA: IEEE, 2008, pp. 410-415.

[21] C. Liu, K. Wu, Y. Xiao, and B. Sun, "Random coverage with guaranteed connectivity: joint scheduling for wireless sensor networks," IEEE Transactions on Parallel and Distributed Systems, vol. 17, no. 6, pp. 562-575, 2006.

[22] Q. Cao, T. Yan, J. Stankovic, and T. Abdelzaher, "Analysis of target detection performance for wireless sensor networks," in Distributed Computing in Sensor Systems. Springer, 2005, pp. 276-292.

[23] S. Tilak, N. B. Abu-Ghazaleh, and W. Heinzelman, "A taxonomy of wireless micro-sensor network models," ACM SIGMOBILE Mobile Computing and Communications Review, vol. 6, no. 2, pp. 28-36, 2002.

[24] Y. Sun, O. Gurewitz, and D. B. Johnson, "RI-MAC: A receiver-initiated asynchronous duty cycle MAC protocol for dynamic traffic loads in wireless sensor networks," in Proceedings of the 6th ACM Conference on Embedded Networked Sensor Systems (SenSys), Raleigh, NC, USA, 4-7 Nov 2008, pp. $1-14$.

[25] Y. Sun, S. Du, O. Gurewitz, and D. B. Johnson, “DW-MAC: A low latency, energy efficient demand-wakeup MAC protocol for wireless sensor networks," in Proceedings of the 9th ACM International Symposium on Mobile Ad Hoc Networking and Computing, Hong Kong SAR, China, 27-20 Aug 2008, pp. 53-62. 
[26] M. Cardei and J. Wu, "Energy-efficient coverage problems in wireless adhoc sensor networks," Computer Communications, vol. 29, no. 4, pp. 413-420, 2006.

[27] C.-F. Hsin and M. Liu, "Network coverage using low duty-cycled sensors: random \& coordinated sleep algorithms," in Proceedings of the 3rd International Symposium on Information Processing in Sensor Networks (IPSN), Berkeley, CA, USA, 26-27 Apr 2004, pp. 433-442.

[28] V. Raghunathan, A. Kansal, J. Hsu, J. Friedman, and M. Srivastava, “Design considerations for solar energy harvesting wireless embedded systems," in Proceedings of the 4th International Symposium on Information Processing in Sensor Networks (IPSN), Los Angeles, California, USA, 25-27 Apr 2005.

[29] N. Khosropour, F. Krummenacher, and M. Kayal, "Fully integrated ultralow power management system for micro-power solar energy harvesting applications," Electronics Letters, vol. 48, no. 6, pp. 338-339, 2012.

[30] L. Atzori, A. Iera, and G. Morabito, "The internet of things: A survey," Computer networks, vol. 54, no. 15, pp. 2787-2805, 2010.

[31] L. Mainetti, L. Patrono, and A. Vilei, "Evolution of wireless sensor networks towards the internet of things: A survey," in 19th International Conference on Software, Telecommunications and Computer Networks (SoftCOM), Adriatic Islands, Split, Croatia, 15-17 Sept 2011, pp. 1-6.

[32] C. Perera, A. Zaslavsky, P. Christen, and D. Georgakopoulos, "Sensing as a service model for smart cities supported by internet of things," Transactions on Emerging Telecommunications Technologies, vol. 25, no. 1, pp. 81-93, 2014.

[33] A. Caragliu, C. Del Bo, and P. Nijkamp, "Smart cities in europe," Journal of Urban Technology, vol. 18, no. 2, pp. 65-82, 2011.

[34] P. W. Bearman, "Vortex shedding from oscillating bluff bodies," Annual review of fluid mechanics, vol. 16, no. 1, pp. 195-222, 1984.

[35] A. Robertson, R. Hoxey, J. Short, L. Burgess, B. Smith, and R. Ko, “Windinduced fatigue loading of tubular steel lighting columns," Wind and Structures, vol. 4, no. 2, pp. 163-176, 2001. 
[36] D. Hall. (2015, May) M62 Lighting Columns Vortex Shedding. [Online]. Available: https://www.youtube.com/watch?v=awtgPCWD58w

[37] R. H. Bhuiyan, R. A. Dougal, and M. Ali, “A miniature energy harvesting device for wireless sensors in electric power system," IEEE Sensors Journal, vol. 10, no. 7, p. 699, 2010.

[38] W. K. Seah, Z. A. Eu, and H.-P. Tan, "Wireless sensor networks powered by ambient energy harvesting (WSN-HEAP) - survey and challenges," in Proceedings of the 1st International Conference on Wireless Communication, Vehicular Technology, Information Theory and Aerospace \& Electronic Systems Technology. Aalborg, Denmark: IEEE, 2009, pp. 1-5.

[39] A. Nasridinov, S.-Y. Ihm, Y.-S. Jeong, and Y.-H. Park, "Event detection in wireless sensor networks: Survey and challenges," in Mobile, Ubiquitous, and Intelligent Computing. Springer, 2014, pp. 585-590.

[40] N. Pantazis, S. A. Nikolidakis, and D. D. Vergados, "Energy-efficient routing protocols in wireless sensor networks: A survey," Communications Surveys $\mathcal{E}$ Tutorials, IEEE, vol. 15, no. 2, pp. 551-591, 2013.

[41] P. M. Wightman and M. A. Labrador, "A3Cov: A new topology construction protocol for connected area coverage in WSN," in Wireless Communications and Networking Conference (WCNC). IEEE, 2011, pp. 522-527.

[42] Y. Cai, M. Li, W. Shu, and M.-Y. Wu, "ACOS: An area-based collaborative sleeping protocol for wireless sensor networks," Ad Hoc E Sensor Wireless Networks, vol. 3, no. 1, pp. 77-97, 2007.

[43] L. Cheng, C. Wu, Y. Zhang, H. Wu, M. Li, and C. Maple, "A survey of localization in wireless sensor network," International Journal of Distributed Sensor Networks, vol. 2012, 2012.

[44] H. Sundani, H. Li, V. Devabhaktuni, M. Alam, and P. Bhattacharya, "Wireless sensor network simulators a survey and comparisons," International Journal of Computer Networks, vol. 2, no. 5, pp. 249-265, 2011.

[45] J. Zhang and V. Varadharajan, "Wireless sensor network key management survey and taxonomy," Journal of Network and Computer Applications, vol. 33, no. 2, pp. 63-75, 2010. 
[46] V. Katiyar, N. Chand, and S. Soni, "Clustering algorithms for heterogeneous wireless sensor network: A survey," International Journal of Advanced Networking and Applications, vol. 2, no. 4, pp. 745-754, 2011.

[47] J. Sen, "A survey on wireless sensor network security," arXiv preprint arXiv:1011.1529, 2010.

[48] J. J. Rodrigues and P. A. Neves, "A survey on IP-Based wireless sensor network solutions," International Journal of Communication Systems, vol. 23, no. 8, pp. 963-981, 2010.

[49] G. Fan and S. Jin, "Coverage problem in wireless sensor network: A survey," Journal of networks, vol. 5, no. 9, pp. 1033-1040, 2010.

[50] D. Christin, P. S. Mogre, and M. Hollick, "Survey on wireless sensor network technologies for industrial automation: The security and quality of service perspectives," Future Internet, vol. 2, no. 2, pp. 96-125, 2010.

[51] D. Martins and H. Guyennet, "Wireless sensor network attacks and security mechanisms: A short survey," in Proceedings of 13th International Conference on Network-Based Information Systems (NBiS). IEEE, 2010, pp. 313-320.

[52] P. Mohanty, S. Panigrahi, N. Sarma, and S. S. Satapathy, "Security issues in wireless sensor network data gathering protocols: A survey," Journal of Theoretical \& Applied Information Technology, vol. 13, 2010.

[53] J. P. Walters, Z. Liang, W. Shi, and V. Chaudhary, "Wireless sensor network security: A survey," Security in distributed, grid, mobile, and pervasive computing, vol. 1, p. 367, 2007.

[54] C.-C. Han, R. Kumar, R. Shea, and M. Srivastava, "Sensor network software update management: A survey," International Journal of Network Management, vol. 15, no. 4, pp. 283-294, 2005.

[55] G. Zhou, T. He, J. Stankovic, T. Abdelzaher et al., “RID: Radio interference detection in wireless sensor networks," in Proceedings of 24th Annual Joint Conference of the IEEE Computer and Communications Societies, vol. 2. IEEE, 2005, pp. 891-901.

[56] J. P. Olds and W. K. Seah, "Design of an active radio frequency powered multi-hop wireless sensor network," in Proceedings of 7th IEEE Conference 
on Industrial Electronics and Applications (ICIEA). Singapore: IEEE, 2012, pp. 1721-1726.

[57] G. Wittenburg, N. Dziengel, S. Adler, Z. Kasmi, M. Ziegert, and J. Schiller, "Cooperative event detection in wireless sensor networks," Communications Magazine, IEEE, vol. 50, no. 12, pp. 124-131, 2012.

[58] S. N. Das and S. Misra, "Event-driven probabilistic topology management in sparse wireless sensor network," Wireless Sensor Systems, IET, vol. 5, no. 4, pp. 210-217, 2015.

[59] G. Wittenburg, N. Dziengel, C. Wartenburger, and J. Schiller, "A system for distributed event detection in wireless sensor networks," in Proceedings of the 9th ACM/IEEE International Conference on Information Processing in Sensor Networks. Stockholm, Sweden: ACM, 2010, pp. 94-104.

[60] F. Martincic and L. Schwiebert, "Distributed event detection in sensor networks," in Proceedings of International Conference on Systems and Networks Communications (ICSNC). Papeete, Tahiti, French Polynesia: IEEE, 2006, p. 43.

[61] B. Krishnamachari and S. Iyengar, "Distributed bayesian algorithms for fault-tolerant event region detection in wireless sensor networks," IEEE Transactions on Networking, vol. 53, no. 3, pp. 241-250, 2004.

[62] X. Luo, M. Dong, and Y. Huang, "On distributed fault-tolerant detection in wireless sensor networks," IEEE Transactions on Computers, vol. 55, no. 1, pp. 58-70, 2006.

[63] S. Bandyopadhyay and E. J. Coyle, "An energy efficient hierarchical clustering algorithm for wireless sensor networks," in Proceedings of the 22nd Annual Joint Conference of the IEEE Computer and Communications Societies (INFOCOM). San Francisco, CA, USA: IEEE, 30 Mar - 3 Apr 2003, pp. 1713-1723.

[64] S. Li, S. H. Son, and J. A. Stankovic, "Event detection services using data service middleware in distributed sensor networks," in Information Processing in Sensor Networks. Springer, 2003, pp. 502-517. 
[65] R. R. Brooks, P. Ramanathan, and A. M. Sayeed, "Distributed target classification and tracking in sensor networks," Proceedings of the IEEE, vol. 91, no. 8, pp. 1163-1171, 2003.

[66] T. He, S. Krishnamurthy, J. A. Stankovic, T. Abdelzaher, L. Luo, R. Stoleru, T. Yan, L. Gu, J. Hui, and B. Krogh, "Energy-efficient surveillance system using wireless sensor networks," in Proceedings of the 2nd international conference on mobile systems, applications, and services. Boston, MA, USA: ACM, 2004, pp. 270-283.

[67] K. Kapitanova, S. H. Son, and K.-D. Kang, “Using fuzzy logic for robust event detection in wireless sensor networks," Ad Hoc Networks, vol. 10, no. 4, pp. 709-722, 2012.

[68] K.-X. Thuc and K. Insoo, “A collaborative event detection scheme using fuzzy logic in clustered wireless sensor networks," AEU-International Journal of Electronics and Communications, vol. 65, no. 5, pp. 485-488, 2011.

[69] Q. Liang and L. Wang, "Event detection in wireless sensor networks using fuzzy logic system," in Computational Intelligence for Homeland Security and Personal Safety, 2005. CIHSPS 2005. Proceedings of the 2005 IEEE International Conference on. IEEE, 2005, pp. 52-55.

[70] M. Sharma and A. K. Singh, "Analysis of Type-2 fuzzy systems for WSN: A Survey," in Intelligent Computing, Networking, and Informatics. Springer, 2014, pp. 1115-1123.

[71] S. Rashid, U. Akram, S. Qaisar, S. A. Khan, and E. Felemban, "Wireless sensor network for distributed event detection based on machine learning," in Proceedings of 2014 IEEE International Conference on Internet of Things (iThings 2014), Green Computing and Communications (GreenCom 2014), and Cyber-Physical-Social Computing (CPSCom 2014). IEEE, 2014, pp. 540-545.

[72] P.-V. Mekikis, G. Athanasiou, and C. Fischione, "A wireless sensor network testbed for event detection in smart homes," in Proceedings of International Conference on Distributed Computing in Sensor Systems (DCOSS). Cambridge, MA, UAS: IEEE, 2013, pp. 321-322.

[73] K. M. Alam, J. Kamruzzaman, G. Karmakar, and M. Murshed, "Priority sensitive event detection in hybrid wireless sensor networks," in Proceed- 
ings of the 21st International Conference on Computer Communications and Networks (ICCCN), München, Germany, 30 Jul - 2 Aug 2012, pp. 1-7.

[74] B. Milic, "Optimal stopping strategies in collaborative event detection in wireless sensor networks," in Proceedings of 4th International Congress on Ultra Modern Telecommunications and Control Systems and Workshops (ICUMT). St. Petersburg, Russia: IEEE, 2012, pp. 842-849.

[75] Y. Zhu, Y. Liu, and L. M. Ni, "Optimizing event detection in low dutycycled sensor networks," Wireless Networks, vol. 18, no. 3, pp. 241-255, 2012.

[76] S. Kavitha and S. Lalitha, "Sleep scheduling for critical event monitoring in wireless sensor networks," International Journal of Advanced Research in Computer and Communication Engineering, vol. 3, no. 1, pp. 4974-4978, 2014.

[77] P. Guo, T. Jiang, Q. Zhang, and K. Zhang, "Sleep scheduling for critical event monitoring in wireless sensor networks," IEEE Transactions on Parallel and Distributed Systems, vol. 23, no. 2, pp. 345-352, 2012.

[78] C. M. Vigorito, D. Ganesan, and A. G. Barto, "Adaptive control of duty cycling in energy-harvesting wireless sensor networks," in Proceedings of 4th Annual IEEE Communications Society Conference on Sensor, Mesh and Ad Hoc Communications and Networks. San Diego, California, USA: IEEE, 2007, pp. 21-30.

[79] Y. Kang, Y. Han, and J. Hu, "A node scheduling based on partition for wsn," in Proceedings of Wireless Telecommunications Symposium (WTS). London, United Kingdom: IEEE, 2012, pp. 1-6.

[80] T. He, S. Krishnamurthy, L. Luo, T. Yan, L. Gu, R. Stoleru, G. Zhou, Q. Cao, P. Vicaire, J. A. Stankovic et al., "VigilNet: An integrated sensor network system for energy-efficient surveillance," ACM Transactions on Sensor Networks (TOSN), vol. 2, no. 1, pp. 1-38, 2006.

[81] A. Keshavarzian, H. Lee, and L. Venkatraman, "Wakeup scheduling in wireless sensor networks," in Proceedings of the 7th ACM international symposium on Mobile ad hoc networking and computing. Florence, Italy: ACM, 2006, pp. 322-333. 
[82] S. Kumar, T. H. Lai, and J. Balogh, "On k-coverage in a mostly sleeping sensor network," in Proceedings of the 10th annual international conference on Mobile computing and networking. Philadelphia, PA, USA: ACM, 2004, pp. 144-158.

[83] D. Tian and N. D. Georganas, "A coverage-preserving node scheduling scheme for large wireless sensor networks," in Proceedings of the 1st ACM international workshop on wireless sensor networks and applications. Atlanta, GA, USA: ACM, 2002, pp. 32-41.

[84] S. Kulkarni, A. Iyer, and C. Rosenberg, "An address-light, integrated MAC and routing protocol for wireless sensor networks," IEEE/ACM Transactions on Networking, vol. 14, no. 4, pp. 793-806, 2006.

[85] W. Ye, J. Heidemann, and D. Estrin, "An energy-efficient mac protocol for wireless sensor networks," in Proceedings of the 21st Annual Joint Conference of the IEEE Computer and Communications Societies, vol. 3. New York, NY, USA: IEEE, 2002, pp. 1567-1576.

[86] H. Karvonen, J. Petäjäjärvi, J. Iinatti, M. Hämäläinen, and C. PomalazaRáez, "A generic wake-up radio based MAC protocol for energy efficient short range communication," in Proceedings of 25th Annual International Sympsoium on Personal, Indoor, and Mobile Radio Communications (PIMRC), 2014, pp. 1-5.

[87] V. Rajendran, K. Obraczka, and J. J. Garcia-Luna-Aceves, “Energy-efficient, collision-free medium access control for wireless sensor networks," Wireless Networks, vol. 12, no. 1, pp. 63-78, 2006.

[88] J. Feng and M. Potkonjak, "Power minimization by separation of control and data radios," in Proceedings of the IEEE CAS Workshop on Wireless Communication and Networking, vol. 9. Pasadena, CA, USA: IEEE, 2002, pp. $112-121$.

[89] H. Yoo, M. Shim, and D. Kim, "Dynamic duty-cycle scheduling schemes for energy-harvesting wireless sensor networks," IEE Communications Letters, vol. 16, no. 2, pp. 202-204, 2012.

[90] J. Polastre, J. Hill, and D. Culler, "Versatile low power media access for wireless sensor networks," in Proceedings of the 2 nd international conference 
on embedded networked sensor systems. Baltimore, MD, USA: ACM, 2004, pp. 95-107.

[91] T. Van Dam and K. Langendoen, "An adaptive energy-efficient MAC protocol for wireless sensor networks," in Proceedings of the 1st international conference on embedded networked sensor systems. Los Angeles, CA, USA: ACM, 2003, pp. 171-180.

[92] A. Sheth, K. Tejaswi, P. Mehta, C. Parekh, R. Bansal, S. Merchant, T. Singh, U. B. Desai, C. A. Thekkath, and K. Toyama, "Senslide: A sensor network based landslide prediction system," in Proceedings of the 3rd international conference on embedded networked sensor systems. San Diego, CA, USA: ACM, 2005, pp. 280-281.

[93] W. B. Heinzelman, A. P. Chandrakasan, and H. Balakrishnan, "An application-specific protocol architecture for wireless microsensor networks," IEEE Transactions on Wireless Communications, vol. 1, no. 4, pp. 660670, 2002.

[94] Z. Yang, K. Ren, and C. Liu, "Efficient data collection with spatial clustering in time constraint wsn applications," in Pervasive Computing and the Networked World. Springer, 2013, pp. 728-742.

[95] M. C. Vuran and I. F. Akyildiz, "Spatial correlation-based collaborative medium access control in wireless sensor networks," IEEE/ACM Transactions onNetworking, vol. 14, no. 2, pp. 316-329, 2006.

[96] K. Jamieson, H. Balakrishnan, and Y. Tay, "Sift: A MAC protocol for eventdriven wireless sensor networks," in Wireless Sensor Networks. Springer, 2006, pp. 260-275.

[97] L. Krishnamachari, D. Estrin, and S. Wicker, "The impact of data aggregation in wireless sensor networks," in Proceedings of 22nd International Conference on Distributed Computing Systems Workshops. Vienna, Austria: IEEE, 2002, pp. 575-578.

[98] C. Intanagonwiwat, D. Estrin, R. Govindan, and J. Heidemann, "Impact of network density on data aggregation in wireless sensor networks," in Proceedings of 22nd International Conference on Distributed Computing Systems. Vienna, Austria: IEEE, 2002, pp. 457-458. 
[99] S. Borzsony, D. Kossmann, and K. Stocker, "The skyline operator," in Proceedings of the 17th International Conference on Data Engineering. Heidelberg, Germany: IEEE, 2-6 Apr 2001, pp. 421-430.

[100] K. Pripužić, H. Belani, and M. Vuković, "Early forest fire detection with sensor networks: Sliding window skylines approach," in Knowledge-Based Intelligent Information and Engineering Systems. Springer, 2008, pp. 725-732.

[101] J. A. Gutierrez, M. Naeve, E. Callaway, M. Bourgeois, V. Mitter, and B. Heile, "Ieee 802.15. 4: a developing standard for low-power low-cost wireless personal area networks," IEEE Network, vol. 15, no. 5, pp. 12-19, 2001.

[102] M.-H. Zayani, N. Aitsaadi, and P. Muhlethaler, "A new opportunistic routing scheme in low duty-cycle wsns for monitoring infrequent events," in Wireless Days (WD), 2014 IFIP. IEEE, 2014, pp. 1-4.

[103] H. Zhang, A. Arora, Y.-r. Choi, and M. G. Gouda, "Reliable bursty convergecast in wireless sensor networks," Computer Communications, vol. 30, no. 13, pp. 2560-2576, 2007.

[104] P. Levis, S. Madden, J. Polastre, R. Szewczyk, K. Whitehouse, A. Woo, D. Gay, J. Hill, M. Welsh, E. Brewer et al., “TinyOS: An operating system for sensor networks," in Ambient intelligence. Springer, 2005, pp. 115-148.

[105] M. Cheng, Y. Chen, and W. K. G. Seah, "Event-driven energy-harvesting wireless sensor network for structural health monitoring," in Proceedings of the 38th Annual IEEE Conference on Local Computer Networks (LCN), Sydney, Australia, 21-24 Oct 2013, p. 9.

[106] S. Kumar, A. Arora, and T.-H. Lai, "On the lifetime analysis of always-on wireless sensor network applications," in Proceedings of 2nd IEEE International Conference on Mobile Adhoc and Sensor Systems. Washington, DC, USA: IEEE, 2005, pp. 3-pp.

[107] N. Xu, S. Rangwala, K. K. Chintalapudi, D. Ganesan, A. Broad, R. Govindan, and D. Estrin, "A wireless sensor network for structural monitoring," in Proceedings of the 2nd international conference on Embedded networked sensor systems. Baltimore, MD, USA: ACM, 2004, pp. 13-24. 
[108] G. Werner-Allen, K. Lorincz, M. Ruiz, O. Marcillo, J. Johnson, J. Lees, and M. Welsh, "Deploying a wireless sensor network on an active volcano," Internet Computing, IEEE, vol. 10, no. 2, pp. 18-25, 2006.

[109] F. Viani, M. Salucci, P. Rocca, G. Oliveri, and A. Massa, "A multi-sensor WSN backbone for museum monitoring and surveillance," in Proceedings of the 6th European Conference onAntennas and Propagation (EUCAP). Prague, Czech Republic: IEEE, 2012, pp. 51-52.

[110] J. M. Gilbert and F. Balouchi, "Comparison of energy harvesting systems for wireless sensor networks," International Journal of Automation and Computing, vol. 5, no. 4, pp. 334-347, 2008.

[111] S. Sudevalayam and P. Kulkarni, "Energy harvesting sensor nodes: Survey and implications," IEEE Communications Surveys $\mathcal{E}$ Tutorials, vol. 13, no. 3, pp. 443-461, 2011.

[112] X. Jiang, J. Polastre, and D. Culler, "Perpetual environmentally powered sensor networks," in Proceedings of Fourth International Symposium on Information Processing in Sensor Networks. Los Angeles, CA, USA: IEEE, 2005, pp. 463-468.

[113] C. Park and P. H. Chou, "Ambimax: Autonomous energy harvesting platform for multi-supply wireless sensor nodes," in Proceedings of 3rd Annual IEEE Communications Society on Sensor and Ad Hoc Communications and Networks, vol. 1. Reston, VA, USA: IEEE, 2006, pp. 168-177.

[114] A. Ammar and D. Reynolds, "An adaptive scheduling scheme for cooperative energy harvesting networks," Journal of Communications and Networks, vol. 17, no. 3, pp. 256-264, 2015.

[115] H. Li, N. Jaggi, and B. Sikdar, "An analytical approach towards cooperative relay scheduling under partial state information," in Processdings of the 31st International Conference on Computer Communications (INFOCOM). IEEE, 2012, pp. 2666-2670.

[116] X. Fafoutis and N. Dragoni, “ODMAC: An on-demand MAC protocol for energy harvesting-wireless sensor networks," in Proceedings of the 8th ACM Symposium on Performance evaluation of wireless ad hoc, sensor, and ubiquitous networks. Miami Beach, FL, USA: ACM, 2011, pp. 49-56. 
[117] G. Park, T. Rosing, M. D. Todd, C. R. Farrar, and W. Hodgkiss, “Energy harvesting for structural health monitoring sensor networks," Journal of Infrastructure Systems, vol. 14, no. 1, pp. 64-79, 2008.

[118] D. Tomicek, Y.-H. Tham, W. K. Seah, and R. Rayudu, “Vibration-powered wireless sensor for structural monitoring during earthquakes," in Proceedings of the 6th International Conference on Structural Health Monitoring of Intelligent Infrastructure. Hong Kong, China: SHMII, 2013, p. 10.

[119] A. Kansal, J. Hsu, S. Zahedi, and M. B. Srivastava, "Power management in energy harvesting sensor networks," ACM Transactions on Embedded Computing Systems (TECS), vol. 6, no. 4, p. 32, 2007.

[120] Q. Tang, Q. He, M. Li, C. Dong, D. Xu, and X. Li, “A self-powered wireless sensing node for event-driven alerting based on a bi-stable vibration energy harvester," in Proceedings of 18th International Conference on SolidState Sensors, Actuators and Microsystems (TRANSDUCERS). IEEE, 2015, pp. 1215-1218.

[121] I. F. Akyildiz, W. Su, Y. Sankarasubramaniam, and E. Cayirci, "A survey on sensor networks," IEEE Communications Magazine, vol. 40, no. 8, pp. 102114, 2002.

[122] J. Yick, B. Mukherjee, and D. Ghosal, "Wireless sensor network survey," Computer networks, vol. 52, no. 12, pp. 2292-2330, 2008.

[123] L. Mottola and G. P. Picco, "Programming wireless sensor networks: Fundamental concepts and state of the art," ACM Computing Surveys (CSUR), vol. 43, no. 3, p. 19, 2011.

[124] M. Li, Y. Liu, and L. Chen, "Nonthreshold-based event detection for 3D environment monitoring in sensor networks," IEEE Transactions on Knowledge and Data Engineering, vol. 20, no. 12, pp. 1699-1711, 2008.

[125] A. P. R. da Silva, M. H. Martins, B. P. Rocha, A. A. Loureiro, L. B. Ruiz, and H. C. Wong, "Decentralized intrusion detection in wireless sensor networks," in Proceedings of the 1st ACM international workshop on Quality of service $\mathcal{E}$ security in wireless and mobile networks. Montreal, Quebec, Canada: ACM, 2005, pp. 16-23. 
[126] B. Sun, L. Osborne, Y. Xiao, and S. Guizani, "Intrusion detection techniques in mobile ad hoc and wireless sensor networks," IEEE Wireless Communications, vol. 14, no. 5, pp. 56-63, 2007.

[127] R. Roman, J. Zhou, and J. Lopez, “Applying intrusion detection systems to wireless sensor networks," in Proceedings of IEEE Consumer Communications and Networking Conference (CCNC), Las Vegas, Nevada, USA, 2006, pp. 640644.

[128] A. Czarlinska, W. Luh, and D. Kundur, "Attacks on sensing in hostile wireless sensor-actuator environments," in Proceedings of the Global Telecommunications Conference (Globecom). Washington, DC, USA: IEEE, 26-30 Dec 2007, pp. 1001-1005.

[129] A. Mahapatro and P. M. Khilar, "Fault diagnosis in wireless sensor networks: A survey," Communications Surveys E Tutorials, IEEE, vol. 15, no. 4, pp. 2000-2026, 2013.

[130] E. Ould-Ahmed-Vall, B. H. Ferri, and G. F. Riley, "Distributed faulttolerance for event detection using heterogeneous wireless sensor networks," Mobile Computing, IEEE Transactions on, vol. 11, no. 12, pp. 19942007, 2012.

[131] R. Jurdak, X. R. Wang, O. Obst, and P. Valencia, "Wireless sensor network anomalies: Diagnosis and detection strategies," in Intelligence-Based Systems Engineering. Springer, 2011, pp. 309-325.

[132] A. Krause, E. Horvitz, A. Kansal, and F. Zhao, "Toward community sensing," in Proceedings of the 7th international conference on Information processing in sensor networks. St. Louis, Missouri, USA: IEEE Computer Society, 2008, pp. 481-492.

[133] S. Dashti, J. Reilly, J. D. Bray, A. Bayen, S. Glaser, E. Mari, and P. J. D. Bray, "iShake: Using personal devices to deliver rapid semi-qualitative earthquake shaking information," GeoEngineering Report, Depart. of Civil and Environ. Engineering, Univ. of California, Berkeley, Feb, vol. 28, 2011.

[134] M. Faulkner, M. Olson, R. Chandy, J. Krause, K. M. Chandy, and A. Krause, "The next big one: Detecting earthquakes and other rare events from 
community-based sensors," in Proceedings of the 10th International Conference on Information Processing in Sensor Networks (IPSN). Chicago, IL, USA: IEEE, 2011, pp. 13-24.

[135] T. Sakaki, M. Okazaki, and Y. Matsuo, "Earthquake shakes twitter users: real-time event detection by social sensors," in Proceedings of the 19th international conference on world wide web. Raleigh, North Carolina, USA: ACM, 2010, pp. 851-860.

[136] M. Cardei and J. Wu, “Coverage in wireless sensor networks," Handbook of Sensor Networks, pp. 422-433, 2004.

[137] P. Santi, "Topology control in wireless ad hoc and sensor networks," ACM Computing Surveys (CSUR), vol. 37, no. 2, pp. 164-194, 2005.

[138] L. Wang and Y. Xiao, "A survey of energy-efficient scheduling mechanisms in sensor networks," Mobile Networks and Applications, vol. 11, no. 5, pp. 723-740, 2006.

[139] A. Ghosh and S. K. Das, "Coverage and connectivity issues in wireless sensor networks: A survey," Pervasive and Mobile Computing, vol. 4, no. 3, pp. 303-334, 2008.

[140] C. Zhu, C. Zheng, L. Shu, and G. Han, "A survey on coverage and connectivity issues in wireless sensor networks," Journal of Network and Computer Applications, vol. 35, no. 2, pp. 619-632, 2012.

[141] R. C. Carrano, D. Passos, L. C. Magalhaes, and C. V. Albuquerque, "Survey and taxonomy of duty cycling mechanisms in wireless sensor networks," IEEE Communications Surveys E Tutorials, vol. 16, no. 1, pp. 181-194, 2014.

[142] R. Kershner, "The number of circles covering a set," American Journal of Mathematics, pp. 665-671, 1939.

[143] K. Kar, S. Banerjee et al., "Node placement for connected coverage in sensor networks," in Proceedings of WiOpt'03: Modeling and Optimization in Mobile, Ad Hoc and Wireless Networks, Sophia-Antipolis, France, 2003.

[144] C.-F. Huang and Y.-C. Tseng, "The coverage problem in a wireless sensor network," Mobile Networks and Applications, vol. 10, no. 4, pp. 519-528, 2005. 
[145] A. Boukerche and X. Fei, "Coverage protocols for detecting fully sponsored sensors in wireless sensor networks," in Proceedings of the 3rd ACM International Workshop on Performance Evaluation of Wireless Ad hoc, Sensor and Ubiquitous Networks (PE-WASUN). Torremolinos, Malaga, Spain: ACM, 6 Oct 2006, pp. 58-65.

[146] M. Younis and K. Akkaya, "Strategies and techniques for node placement in wireless sensor networks: A survey," Ad Hoc Networks, vol. 6, no. 4, pp. 621-655, 2008.

[147] A. Boukerche, X. Fei, and R. B. Araujo, "A coverage-preserving and hole tolerant based scheme for the irregular sensing range in wireless sensor networks," in Proceedings of the Global Telecommunications Conference (GLOBECOM). San Francisco, CA, USA: IEEE, 27 Nov - 1 Dec 2006, pp. 1-5.

[148] A. Boukerche and X. Fei, "A coverage-preserving scheme for wireless sensor network with irregular sensing range," Ad hoc networks, vol. 5, no. 8, pp. 1303-1316, 2007.

[149] V. Zalyubovskiy, A. Erzin, S. Astrakov, and H. Choo, "Energy-efficient area coverage by sensors with adjustable ranges," Sensors, vol. 9, no. 4, pp. 24462460, 2009.

[150] J. Carle and D. Simplot-Ryl, "Energy-efficient area monitoring for sensor networks," Computer, vol. 37, no. 2, pp. 40-46, 2004.

[151] F. Pedraza, A. L. Medaglia, and A. Garcia, "Efficient coverage algorithms for wireless sensor networks," in Proceedings of Systems and Information Engineering Design Symposium. Charlottesville, Virginia, USA: IEEE, 2006, pp. 78-83.

[152] J. Lu and T. Suda, "Coverage-aware self-scheduling in sensor networks," in Proceedings 18th Annual Workshop on Computer Communications. Dana Point, California, USA: IEEE, 2003, pp. 117-123.

[153] X. Bai, S. Kumar, D. Xuan, Z. Yun, and T. H. Lai, "Deploying wireless sensors to achieve both coverage and connectivity," in Proceedings of the 7th ACM International Symposium on Mobile Ad Hoc Networking and Computing (MobiHoc). Florence, Italy: ACM, 22-25 May 2006, pp. 131-142. 
[154] X. Wang, G. Xing, Y. Zhang, C. Lu, R. Pless, and C. Gill, "Integrated coverage and connectivity configuration in wireless sensor networks," in Proceedings of the ACM SenSys, Los Angeles, California, USA, 5-7 Nov 2003, pp. 28-39.

[155] B. Chen, K. Jamieson, H. Balakrishnan, and R. Morris, “Span: An energyefficient coordination algorithm for topology maintenance in ad hoc wireless networks," Wireless Networks, vol. 8, no. 5, pp. 481-494, 2002.

[156] H. Zhang and J. C. Hou, "Maintaining sensing coverage and connectivity in large sensor networks," Ad Hoc E Sensor Wireless Networks, vol. 1, no. 1-2, pp. 89-124, 2005.

[157] Y. Xu, J. Heidemann, and D. Estrin, “Geography-informed energy conservation for ad hoc routing," in Proceedings of the 7th annual international conference on Mobile computing and networking. ACM, 2001, pp. 70-84.

[158] F. Ye, G. Zhong, J. Cheng, S. Lu, and L. Zhang, "PEAS: A robust energy conserving protocol for long-lived sensor networks," in Proceedings of the 23rd International Conference on Distributed Computing Systems. Providence, Rhode Island, USA: IEEE, 2003, pp. 28-37.

[159] C.-F. Huang, Y.-C. Tseng, and H.-L. Wu, "Distributed protocols for ensuring both coverage and connectivity of a wireless sensor network," ACM Transactions on Sensor Networks (TOSN), vol. 3, no. 1, p. 5, 2007.

[160] G. S. Kasbekar, Y. Bejerano, and S. Sarkar, "Lifetime and coverage guarantees through distributed coordinate-free sensor activation," IEEE/ACM Transactions on Networking, vol. 19, no. 2, pp. 470-483, 2011.

[161] B. Gaudette, V. Hanumaiah, M. Krunz, and S. Vrudhula, "Maximizing quality of coverage under connectivity constraints in solar-powered active wireless sensor networks," ACM Transactions on Sensor Networks (TOSN), vol. 10, no. 4, p. 59, 2014.

[162] C. Yang and K.-W. Chin, "Novel algorithms for complete targets coverage in energy harvesting wireless sensor networks," IEEE Communications Letters, vol. 18, no. 1, pp. 118-121, 2014. 
[163] D. J. Yeager, P. S. Powledge, R. Prasad, D. Wetherall, and J. R. Smith, "Wirelessly-charged UHF tags for sensor data collection," in Proceedings of the IEEE International Conference on RFID, Las Vegas, Nevada, USA, 16-17 April 2008, pp. 320-327.

[164] S. He, J. Chen, F. Jiang, D. K. Yau, G. Xing, and Y. Sun, "Energy provisioning in wireless rechargeable sensor networks," IEEE Transactions on Mobile Computing, vol. 12, no. 10, pp. 1931-1942, 2013.

[165] T. Jiang, G. V. Merrett, and N. R. Harris, “Opportunistic energy trading between co-located energy-harvesting wireless sensor networks," in Proceedings of the 1st International Workshop on Energy Neutral Sensing Systems, Rome, Italy, 14 Nov 2013.

[166] A. Castagnetti, A. Pegatoquet, T. N. Le, and M. Auguin, “A joint duty-cycle and transmission power management for energy harvesting WSN," IEEE Transactions on Industrial Informatics, vol. 10, no. 2, pp. 928-936, 2014.

[167] M. Magno, D. Boyle, D. Brunelli, E. Popovici, and L. Benini, “Ensuring survivability of resource-intensive sensor networks through ultra-low power overlays," IEEE Transactions on Industrial Informatics, vol. 10, no. 2, pp. 946956, 2014.

[168] A. Bader, K. Abed-Meraim, and M.-S. Alouini, "Beaconless geo-routing under the spotlight: Practical link models and application scenarios," IEEE Systems Journal, p. 14, 2015.

[169] F. Cadger, K. Curran, J. Santos, and S. Moffett, "A survey of geographical routing in wireless ad-hoc networks," IEEE Communications Surveys $\mathcal{E}$ Tutorials, vol. 15, no. 2, pp. 621-653, 2013.

[170] J. Sanchez, P. M. Ruiz, R. Marin-Perez et al., "Beacon-less geographic routing made practical: challenges, design guidelines, and protocols," IEEE Communications Magazine, vol. 47, no. 8, pp. 85-91, 2009.

[171] J. N. Al-Karaki and A. E. Kamal, "Routing techniques in wireless sensor networks: A survey," Wireless communications, IEEE, vol. 11, no. 6, pp. 6-28, 2004. 
[172] M. Mauve, J. Widmer, and H. Hartenstein, "A survey on position-based routing in mobile ad hoc networks," IEEE Network, vol. 15, no. 6, pp. 30-39, 2001.

[173] Y.-B. Ko and N. H. Vaidya, "Location-aided routing (LAR) in mobile ad hoc networks," Wireless Networks, vol. 6, no. 4, pp. 307-321, 2000.

[174] P. Basu and C.-K. Chau, "Opportunistic forwarding in wireless networks with duty cycling," in Proceedings of the third ACM Workshop on Challenged Networks. ACM, 2008, pp. 19-26.

[175] E. Ghadimi, O. Landsiedel, P. Soldati, and M. Johansson, “A metric for opportunistic routing in duty cycled wireless sensor networks," in Proceedings of the IEEE SECON, Seoul, Korea, 18-21 June 2012, pp. 335-343.

[176] S. Biswas and R. Morris, "ExOR: opportunistic multi-hop routing for wireless networks," in ACM SIGCOMM Computer Communication Review, vol. 35, no. 4, 2005, pp. 133-144.

[177] S. Chachulski, M. Jennings, S. Katti, and D. Katabi, "Trading structure for randomness in wireless opportunistic routing," ACM SIGCOMM Computer Communication Review, vol. 37, no. 4, 2007.

[178] S. Giordano and I. Stojmenovic, "Position based routing algorithms for ad hoc networks: A taxonomy," in Ad hoc wireless networking. Springer, 2004, pp. 103-136.

[179] H. Füßler, J. Widmer, M. Käsemann, M. Mauve, and H. Hartenstein, "Contention-based forwarding for mobile ad hoc networks," Ad Hoc Networks, vol. 1, no. 4, pp. 351-369, 2003.

[180] M. Heissenbüttel, T. Braun, T. Bernoulli, and M. WäLchli, “BLR: Beaconless routing algorithm for mobile ad hoc networks," Computer communications, vol. 27, no. 11, pp. 1076-1086, 2004.

[181] J. Sanchez, R. Marin-Perez, and P. M. Ruiz, "BOSS: Beacon-less on demand strategy for geographic routing inwireless sensor networks," in Proceedings of IEEE International Conference on Mobile Adhoc and Sensor Systems, Pisa, Italy, 8-11 October 2007, pp. 1-10. 
[182] M. Rossi, N. Bui, and M. Zorzi, "Cost-and collision-minimizing forwarding schemes for wireless sensor networks: Design, analysis and experimental validation," IEEE Transactions on Mobile Computing, vol. 8, no. 3, pp. 322337, 2009.

[183] T. Aguilar, S.-J. Syue, V. Gauthier, H. Afifi, and C.-L. Wang, “CoopGeo: A beaconless geographic cross-layer protocol for cooperative wireless ad hoc networks," IEEE Transactions on Wireless Communications, vol. 10, no. 8, pp. 2554-2565, 2011.

[184] C.-L. Wang and S.-J. Syue, "A geographic-based approach to relay selection for wireless ad hoc relay networks," in 69th IEEE Vehicular Technology Conference. IEEE, 2009, pp. 1-5.

[185] M. Zorzi and R. R. Rao, “Geographic random forwarding (GeRaF) for ad hoc and sensor networks: energy and latency performance," IEEE Transactions on Mobile Computing, vol. 2, no. 4, pp. 349-365, 2003.

[186] A. Odorizzi and G. Mazzini, “M-GeRaF: A reliable random forwarding geographic routing protocol in multisink ad hoc and sensor networks," in Intelligent Signal Processing and Communication Systems, 2007. ISPACS 2007. International Symposium on. IEEE, 2007, pp. 416-419.

[187] D. Ferrara, L. Galluccio, A. Leonardi, G. Morabito, and S. Palazzo, "MACRO: An integrated MAC/routing protocol for geographic forwarding in wireless sensor networks," in Proceedings of INFOCOM 2015: 24th Annual Joint Conference of the IEEE Computer and Communications Societies, vol. 3. IEEE, 2005, pp. 1770-1781.

[188] T. He, B. M. Blum, Q. Cao, J. A. Stankovic, S. H. Son, and T. F. Abdelzaher, "Robust and timely communication over highly dynamic sensor networks," Real-Time Systems, vol. 37, no. 3, pp. 261-289, 2007.

[189] O. Landsiedel, E. Ghadimi, S. Duquennoy, and M. Johansson, "Low power, low delay: opportunistic routing meets duty cycling," in ACM/IEEE 11th International Conference on Information Processing in Sensor Networks (IPSN). IEEE, 2012, pp. 185-196.

[190] X. Zhang, F. Yan, L. Tao, and D. K. Sung, “Optimal candidate set for opportunistic routing in asynchronous wireless sensor networks," in Proceedings 
of the 23rd International Conference on Computer Communication and Networks (ICCCN), Shanghai, China, 4-7 Aug 2014, pp. 1-8.

[191] S. Guo, L. He, Y. Gu, B. Jiang, and T. He, “Opportunistic flooding in lowduty-cycle wireless sensor networks with unreliable links," IEEE Transactions on Computers, vol. 63, no. 11, pp. 2787-2802, 2014.

[192] P. Kyasanur, R. R. Choudhury, and I. Gupta, "Smart Gossip: An adaptive gossip-based broadcasting service for sensor networks," in IEEE International Conference on Mobile Ad Hoc and Sensor Systems. IEEE, 2006, pp. 91100.

[193] P. Levis, N. Patel, D. Culler, and S. Shenker, "Trickle: A self-regulating algorithm for code propagation and maintenance in wireless sensor networks," in Procedings of the 1st USENIX/ACM Symp. on Networked Systems Design and Implementation, 2004.

[194] J. Yang and Z. Fei, "HDAR: Hole detection and adaptive geographic routing for ad hoc networks," in Proceedings of 19th International Conference on Computer Communications and Networks (ICCCN), Zurich, Switzerland, 2-5 Aug 2010, pp. 1-6.

[195] J. Yang and Z. Fei, "ITGR: intermediate target based geographic routing," in Proceedings of 19th International Conference on Computer Communications and Networks (ICCCN). IEEE, 2010, pp. 1-6.

[196] T. Lee, C. Qiao, M. Demirbas, and J. Xu, "ABC: A simple geographic forwarding scheme capable of bypassing routing holes in sensor networks," in Proceedings of 17th International Conference on Computer Communications and Networks (ICCCN), St. Thomas U.S. Virgin Islands, 3-7 Aug 2008, pp. 1-8.

[197] Advanticsys. (2015, March) MTM-CM5000-MSP. [Online]. Available: http:/ / www.advanticsys.com/shop/mtmcm5000msp-p-14.html

[198] Unicomp. (2016, May) UCMote Mini. [Online]. Available: http://www. ucmote.com/en/products/1/3/ucmote-mini

[199] H.-Y. Zhou, D.-Y. Luo, Y. Gao, and D.-C. Zuo, "Modeling of node energy consumption for wireless sensor networks," Wireless Sensor Network, vol. 3, no. 1 , p. 18, 2011. 
[200] C. Alippi, R. Camplani, C. Galperti, and M. Roveri, "Effective design of WSNs: from the lab to the real world," in Proceedings of the 3rd International Conference on Sensing Technology (ICST), Tainan, Taiwan, 30 Nov - 3 Dec 2008, pp. 1-9.

[201] Scalable Network Technologies, “The QualNet ${ }^{\circledR}$ Communications Simulation Platform," 2011. [Online]. Available: http://web.scalable-networks. com/content/qualnet

[202] NIWA. (2015, March) Solarview. [Online]. Available: http://solarview. niwa.co.nz

[203] Futurlec. (2015, March) Solar cells. [Online]. Available: http://www. futurlec.com/Solar_Cell.shtml

[204] I. Khemapech, A. Miller, and I. Duncan, "A survey of transmission power control in wireless sensor networks," in Proceedings of the 8th Annual Postgraduate Symposium on the Convergence of Telecommunications, Networking and Broadcasting. Citeseer, 2007, pp. 15-20.

[205] R. W. Coutinho, A. Boukerche, L. F. Vieira, and A. A. Loureiro, "Transmission power control-based opportunistic routing for wireless sensor networks," in Proceedings of the 17th ACM international conference on Modeling, analysis and simulation of wireless and mobile systems. ACM, 2014, pp. 219226.

[206] K. E. Nolan, W. Guibene, and M. Y. Kelly, "An evaluation of low power wide area network technologies for the Internet of Things," in Proceedings of the International Wireless Communications and Mobile Computing Conference (IWCMC), Paphos, Cyprus, 5-9 Sept 2016, pp. 439-444.

[207] X. Fei, A. Boukerche, and R. B. Araujo, “Irregular sensing range detection model for coverage based protocols in wireless sensor networks," in Proceedings of the IEEE Global Telecommunications Conference. IEEE, 2009, pp. $1-6$.

[208] K. Derr and M. Manic, "Wireless sensor networks-node localization for various industry problems," IEEE Transactions on Industrial Informatics, vol. 11, no. 3, pp. 752-762, 2015. 
[209] RSComponents. (2015, March) Duracell Staycharged NiMH AA Rechargeable Battery, 2400mAh. [Online]. Available: http://uk.rs-online.com/ web/p/aa-rechargeable-batteries/7915987/

[210] H. Shao, C.-Y. Tsui, and W.-H. Ki, "The design of a micro power management system for applications using photovoltaic cells with the maximum output power control," IEEE Transactions on Very Large Scale Integration (VLSI) Systems, vol. 17, no. 8, pp. 1138-1142, 2009.

[211] Y. Qiu, C. Van Liempd, B. Op het Veld, P. G. Blanken, and C. Van Hoof, " $5 \mu \mathrm{w}$-to-10mw input power range inductive boost converter for indoor photovoltaic energy harvesting with integrated maximum power point tracking algorithm," in Proceedings of IEEE International Solid-State Circuits Conference Digest of Technical Papers (ISSCC), San Francisco, CA, USA, 20-24 Feb 2011, pp. 118-120.

[212] J. Kim, J. Kim, and C. Kim, “A regulated charge pump with a low-power integrated optimum power point tracking algorithm for indoor solar energy harvesting," IEEE Transactions on Circuits and Systems II: Express Briefs, vol. 58, no. 12, pp. 802-806, 2011.

[213] J. Yin, X. Wang, and D. P. Agrawal, “Optimal packet size in error-prone channel for IEEE 802.11 distributed coordination function," in Proceedings of IEEE Wireless Communications and Networking Conference, Atlanta, GA, USA, 21-25 March 2004, pp. 1654-1659.

[214] D. Chen, J. Deng, and P. K. Varshney, "Selection of a forwarding area for contention-based geographic forwarding in wireless multi-hop networks," IEEE Transactions on Vehicular Technology, vol. 56, no. 5, pp. 3111-3122, 2007.

[215] A. Koubaa, M. Alves, B. Nefzi, and Y.-Q. Song, "Improving the IEEE 802.15. 4 slotted CSMA/CA MAC for time-critical events in wireless sensor networks," Repositório Científico do Instituto Politécnico do Porto, 2006.

[216] E. F. Nakamura, A. A. Loureiro, and A. C. Frery, "Information fusion for wireless sensor networks: Methods, models, and classifications," ACM Computing Surveys (CSUR), vol. 39, no. 3, p. 9, 2007.

[217] C. Yang and K.-W. Chin, "A novel distributed algorithm for complete targets coverage in energy harvesting wireless sensor networks," in Proceed- 
ings of IEEE International Conference on Communications (ICC), Sydney, Australia, 10-14 June 2014, pp. 361-366.

[218] G. Xing, X. Wang, Y. Zhang, C. Lu, R. Pless, and C. Gill, “Integrated coverage and connectivity configuration for energy conservation in sensor networks," ACM Transactions on Sensor Networks (TOSN), vol. 1, no. 1, pp. 3672, 2005.

[219] H. M. Ammari, "3D-kCov-ComFor: An Energy-Efficient Framework for Composite Forwarding in Three-Dimensional Duty-Cycled k-Covered Wireless Sensor Networks," ACM Transactions on Sensor Networks (TOSN), vol. 12 , no. 4 , p. $35,2016$.

[220] G. Anastasi, M. Conti, M. Di Francesco, and A. Passarella, "Energy conservation in wireless sensor networks: A survey," Ad Hoc Networks, vol. 7, no. 3, pp. 537-568, 2009. 